\title{
Regressão não-paramétrica com erros correlacionados via ondaletas
}

\author{
Rogério de Faria Porto
}

TESE APRESENTADA

$\mathrm{AO}$

Instituto DE MATEMÁticA E EstatísticA

DA

UNIVERSIDADE DE SÃo PAUlO

PARA

OBTENÇÃO DO TÍTULO

$\mathrm{DE}$

DOUTOR EM CiÊNCIAS

\author{
Programa: Estatística \\ Orientador: Prof. Dr. Pedro Alberto Morettin
}

Durante o desenvolvimento deste trabalho o autor recebeu auxílio financeiro do CNPq e do Banco do Brasil

São Paulo, outubro de 2008 



\section{Regressão não-paramétrica com erros correlacionados via ondaletas}

Este exemplar corresponde à redação final da dissertação/tese devidamente corrigida

e defendida por Rogério de Faria Porto e aprovada pela Comissão Julgadora.

Banca Examinadora:

- Prof. Dr. Pedro Alberto Morettin (orientador) - IME-USP.

- Profa. Dra. Clélia Maria de Castro Toloi - IME-USP.

- Profa. Dra. Eliana Zandonade - UFES.

- Profa. Dra. Sílvia Regina Costa Lopes - UFRGS.

- Prof. Dr. Aluísio de Souza Pinheiro - IMECC-UNICAMP. 



\section{Agradecimentos}

Aos meus professores, especialmente ao professor Pedro Alberto Morettin pela excelente e profissional orientação; to professor Donald B. Percival for the opportunity and his foreign advising during my very good stay in Seattle; à professora Elisete da Conceição Quintaneiro Aubin pela dedicação e colaboração. Aos professores Hilton Machado e Édina Miazaki pelos constantes incentivo e apoio antes e durante o doutorado; ao professor Aluísio Pinheiro pelas sugestões bibliográficas e à Véronique Delouille pelo seu programa em MATLAB.

Ao Banco do Brasil pela liberação para realizar este doutorado, pelo apoio financeiro e pelo fornecimento dos dados de recuperação de créditos; ao CNPq pelo apoio financeiro no Brasil e nos Estados Unidos.

Aos meus colegas e amigos do IME-USP, especialmente João, Gladys e Raydonal, pelo incentivo e colaboração; ao Iran pela inspiração quando fez mestrado na UnB morando em Goiânia, pelo apoio inicial em São Paulo e pela companhia nos estudos. Aos colegas que conheci na University of Washington, em Seattle.

Aos meus colegas de trabalho do Banco do Brasil, especialmente ao Marcelo Souza, pela orientação técnica e apoio na escolha do tema, e ao Gláucio Henrique e sua equipe pela ajuda no fornecimento dos dados.

Aos meus familiares, especialmente à minha esposa Denise e meus filhos Pedro, Débora e Danielle pelo amor, apoio, companhia, paciência e compreensão durante o doutorado; também à minha mãe Diva, pela paciência e compreensão. 


\section{Resumo}

Nesta tese, são obtidas taxas de convergência a zero, do risco de estimação obtido com regressão não-paramétrica via ondaletas, quando há erros correlacionados. Quatro métodos de regressão nãoparamétrica via ondaletas, com delineamento desigualmente espaçado são estudados na presença de erros correlacionados, oriundos de processos estocásticos. São apresentadas condições sobre os erros e adaptações aos procedimentos necessárias à obtenção de taxas de convergência quase minimax, para os estimadores. Sempre que possível são obtidas taxas de convergência para os estimadores no domínio da função, sob condições bastante gerais a respeito da função a ser estimada, do delineamento e da correlação dos erros. Mediante estudos de simulação, são avaliados os comportamentos de alguns métodos propostos quando aplicados a amostras finitas. Em geral sugere-se usar um dos procedimentos estudados, porém aplicando-se limiares por níveis. Como a estimação da variância dos coeficientes de detalhes pode ser problemática em alguns casos, também se propõe um procedimento iterativo semi-paramétrico geral para métodos que utilizam ondaletas, na presença de erros em séries temporais.

Palavras-chave: regressão não-paramétrica, ondaletas, autocorrelação, erros em séries temporais, ondaletas deformadas, ondaletas adaptativas, lifting, estimação semi-paramética. 


\section{Abstract}

In this thesis, rates of convergence to zero are obtained for the estimation risk, for non-parametric regression using wavelets, when the errors are correlated. Four non-parametric regression methods using wavelets, with un-equally spaced design are studied in the presence of correlated errors, that come from stochastic processes. Conditions on the errors and adaptrations to the procedures are presented, so that the estimators achieve quasi-minimax rates of convergence. Whenever is possible, rates of convergence are obtained for the estimators in the domain of the function, under mild conditions on the function to be estimated, on the design and on the error correlation. Through simulation studies, the behavior of some of the proposed methods is evaluated, when used on finite samples. Generally, it is suggested to use one of the studied methods, however applying thresholds by level. Since the estimation of the detail coefficients can be difficult in some cases, it is also proposed a general semi-parametric iterative procedure, for wavelet methods in the presence of time-series errors.

Keywords: non-parametric regression, wavelets, autocorrelation, time-series errors, warped wavelets, design-adapted wavelets, lifting, semi-parametric estimation. 


\section{Sumário}

Lista de Abreviaturas $\quad$ xi

Lista de Símbolos $\quad$ xiii

Lista de Figuras $\quad$ xv

Lista de Tabelas $\quad$ xix

1 Introdução 1

2 Preliminares $\quad 5$

2.1 Métodos Usando Ondaletas . . . . . . . . . . . . . . . . . . . . . . 5

2.2 Delineamento Uniforme $\ldots \ldots \ldots \ldots \ldots$

2.3 Ondaletas Deformadas . . . . . . . . . . . . . . . . . . . . . . 10

2.4 Ondaletas Adaptativas e Lifting . . . . . . . . . . . . . . . . . . . . 12

2.5 Esquema de Lifting . . . . . . . . . . . . . . . . . . . . . . 15

$2.5 .1 \quad$ Passo de Previsão . . . . . . . . . . . . . . . . . . . . . . 16

$2.5 .2 \quad$ Passo de Atualização . . . . . . . . . . . . . . . . . . . . . 17

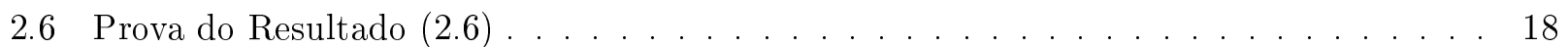

3 Delineamento Fixo $\quad 19$

3.1 Modelo e Procedimento . . . . . . . . . . . . . . . . . . . . . . 19

3.2 Suposições e Resultados Gerais . . . . . . . . . . . . . . . . . . . . . . 21

3.3 Discussão e Implementação Prática . . . . . . . . . . . . . . . . . . . . . . 22

3.3.1 Simulações com Amostras Finitas . . . . . . . . . . . . . . . . . . . 24 
$3.3 .2 \quad$ Aplicação . . . . . . . . . . . . . . . . . . . . . . 25

3.4 Provas . . . . . . . . . . . . . . . . . . . . . . . . . . . . 29

$3.4 .1 \quad$ Prova da Proposição $1 \ldots \ldots \ldots \ldots \ldots$

3.4 .2 Prova do Resultado $(3.8) \ldots \ldots \ldots \ldots \ldots \ldots \ldots$

4 Delineamentos Aleatórios Uniforme e Jittered 33

4.1 Introdução . . . . . . . . . . . . . . . . . . . . . . . . . 33

4.2 Regressão via Ondaletas com Delin. Aleat. e Erros Correlac. . . . . . . . . . . . . . . 34

4.3 Simulações . . . . . . . . . . . . . . . . . . . . . . . . 36

4.4 Aplicação . . . . . . . . . . . . . . . . . . . . . . . 39

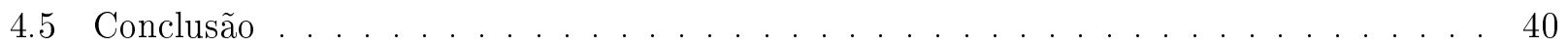

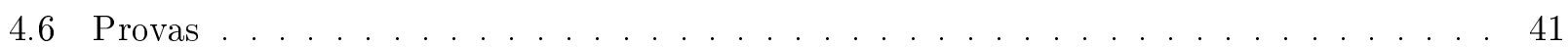

4.6 .1 Prova da Proposição $2 \ldots \ldots \ldots \ldots \ldots \ldots$. . . . . . . . . . . . 41

$4.6 .2 \quad$ Prova da Proposição $3 \ldots \ldots$. . . . . . . . . . . . . . . . . . . 42

4.6 .3 Prova do Teorema $3 \ldots \ldots \ldots \ldots \ldots \ldots$

4.6 .4 Prova do Corolário $1 \ldots \ldots \ldots \ldots \ldots \ldots$

5 Delineamento Aleatório Geral $\quad 57$

5.1 Procedimento Usando Ondaletas Deformadas . . . . . . . . . . . . . . . . . . 57

$5.1 .1 \quad$ Resultados Principais . . . . . . . . . . . . . . . . . . 59

5.1 .2 Comentários . . . . . . . . . . . . . . . . . . . . . 61

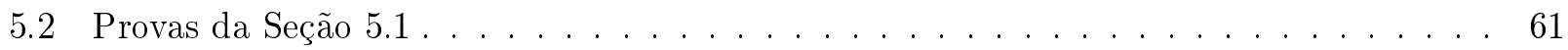

5.2 .1 Prova da Proposição $4 \ldots \ldots \ldots \ldots$. . . . . . . . . . . . . . 61

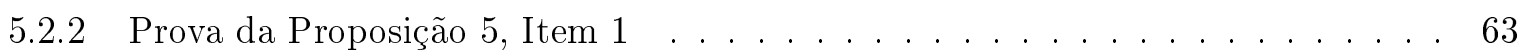

5.2 .3 Prova da Proposição 5 , Item $2 \ldots \ldots \ldots \ldots$. . . . . . . . . . . 65

5.3 Procedimento Usando Ondaletas Adaptativas de Haar . . . . . . . . . . . . . . . 67

5.3 .1 Melhor Estimador Linear _. . . . . . . . . . . . . . . . . 68

5.3 .2 Estimador Não-Linear . . . . . . . . . . . . . . . . . . . 70

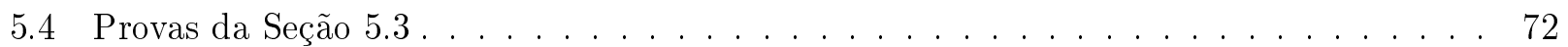




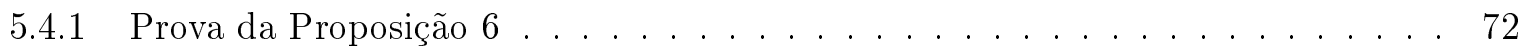

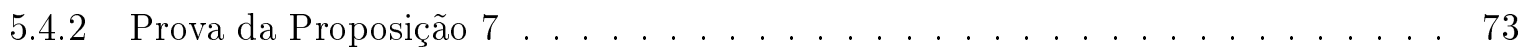

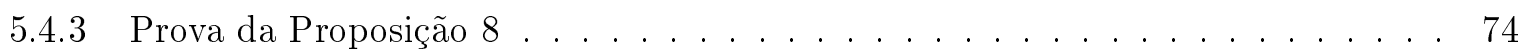

5.4 .4 Prova da Proposição $9 \ldots \ldots \ldots \ldots \ldots \ldots \ldots$

5.5 Procedimento Usando Ondaletas Adaptativas Suaves (Lifting) . . . . . . . . . . 78

5.5.1 Alguns Resultados Sobre os Coeficientes de Ondaletas . . . . . . . . . . . 79

5.5 .2 Estimadores Linear e Não-Linear ～. . . . . . . . . . . . . . . . . . 80

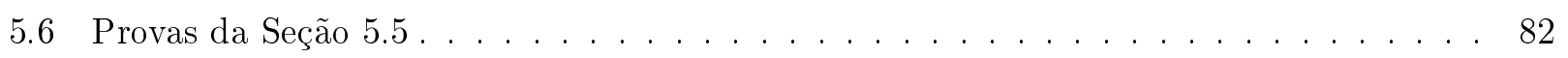

$5.6 .1 \quad$ Prova da Proposição $10 \ldots \ldots \ldots$. . . . . . . . . . . . . . . . . 82

$5.6 .2 \quad$ Prova da Proposição $11 \ldots \ldots \ldots$. . . . . . . . . . . . . . . . . 86

5.7 Aplicações . . . . . . . . . . . . . . . . . . . . . . 86

5.7 .1 Proporção Recuperada de Dívidas . . . . . . . . . . . . . . . 86

5.7 .2 Magnitude de Cefeidas . . . . . . . . . . . . . . . . . . . 89

6 Procedimento Semi-paramétrico $\quad 93$

6.1 Modelo e Suposições . . . . . . . . . . . . . . . . . . . . . . . . . 93

6.2 Procedimento . . . . . . . . . . . . . . . . . . . . . . 94

6.3 Simulações. . . . . . . . . . . . . . . . . . . . . . . 96

6.3 .1 Resultados . . . . . . . . . . . . . . . . . . . . . 98

6.3 .2 Comentários . . . . . . . . . . . . . . . . . . . . . . 102

6.4 Aplicação . . . . . . . . . . . . . . . . . . . . . . . . . 103

7 Conclusões $\quad 107$

$\begin{array}{ll}\text { Referências Bibliográficas } & 109\end{array}$ 


\section{Lista de Abreviaturas}

i.i.d. Independente(s) e identicamente distribuído(s) 8

$\operatorname{AR}(p) \quad$ (processo) autorregressivo de ordem $p$

SURE Estimador Não-viesado de Stein do Risco (Stein Unbiased Risk Estimate)

SNR Razão Sinal-Ruído (Signal-to-Noise Ratio) 36

MSE Erro Quadrático Médio (Mean Square Error)

IMSE $\quad$ Erro Quadrático Médio Integrado (Integrated Mean Square Error) 7

q.c. quase certamente 11

$R_{p}(f, \hat{f}) \quad$ Risco global de norma $L_{p}$, de um estimador $\hat{f}$ de uma função $f \quad 2$

MAD Desvio Mediano Absoluto (Median Absolute Deviation) 9 


\section{Lista de Símbolos}

\begin{tabular}{|c|c|}
\hline $\mathbb{N}$ & Conjunto dos números naturais \\
\hline $\mathbb{Z}$ & Conjunto dos números inteiros \\
\hline $\mathbb{R}$ & Conjunto dos números reais \\
\hline$\oplus$ & Soma direta de dois subespaços \\
\hline$\perp$ & Ortogonalidade entre dois subespaços \\
\hline$\langle\cdot, \cdot\rangle$ & Produto interno \\
\hline$f^{(m)}$ & Derivada de ordem $m$ de uma função $f$ \\
\hline$\lfloor\alpha\rfloor$ & Maior inteiro menor que $\alpha$ \\
\hline$\Lambda^{\alpha}(M, B, m)$ & $\begin{array}{l}\text { Classe de Hölder por partes com até } m \text { pontos de descontinuidade } \\
\text { e parâmetro de regularidade } \alpha\end{array}$ \\
\hline$\Lambda^{1}(h)$ & Classe de Lipschitz, com constante $h$ \\
\hline$\eta_{H}(d, \lambda)$ & Função Limiar Duro (Hard threshold), com argumento $d$ e limiar $\lambda$ \\
\hline$\eta_{S}(d, \lambda)$ & Função Limiar Suave (Soft threshold), com argumento $d$ e limiar $\lambda$ \\
\hline$I(\cdot)$ & Função indicadora \\
\hline$W$ & Matriz da transformada discreta de ondaletas \\
\hline$W^{T}$ & Transposta de uma matriz $W$ \\
\hline$H^{*}$ & Transposta hermitiana de uma matriz $H$ \\
\hline$H^{-1}$ & Inversa de uma matriz $H$ \\
\hline $\operatorname{med}\left\{x_{1}, \ldots, x_{n}\right\}$ & Mediana dos valores $x_{1}, \ldots, x_{n}$ \\
\hline $\operatorname{sgn}(x)$ & Sinal do número real $x$ \\
\hline$f \circ g$ & Composição de duas funções $f$ e $g$ \\
\hline$\|f\|_{p}$ & Norma $L_{p}$ de uma função $f$ \\
\hline$|d|$ ou $|I|$ & $\begin{array}{l}\text { Valor absoluto de um número } d \text { (ou do valor de uma função), ou } \\
\text { comprimento de um intervalo } I \text {. }\end{array}$ \\
\hline$\sigma(I)$ & Sigma-álgebra gerada por $I$ \\
\hline $\operatorname{ess.sup}\{f\}$ & Supremo essencial de uma função $f$ \\
\hline $\operatorname{supp}\{f\}$ & Suporte de uma função $f$ \\
\hline
\end{tabular}




\section{Lista de Figuras}

2.1 Transformadas de ondaletas de análise (esquerda) e síntese (direita) em termos de passos de lifting: $\left(\tilde{H}_{j}, \tilde{G}_{j}\right)$ são alguns filtros iniciais muito simples. No nosso caso, eles são filtros da transformada de ondaletas de Haar não balanceada. A estes filtros seguem-se passos de previsão $P_{j}$ e atualização $U_{j}$. O primeiro aumenta a suavidade de $\phi_{j_{0}, k}$, onde o último pode prover um maior número de momentos nulos, ou aumentar a estabilidade da transformada de ondaletas. . . . . . . . . . . . . . . . . 16

3.1 Razão estimada $C(n)$ entre os riscos quadráticos de norma $L_{2}$ dos dados de memória longa (coeficientes de Hurst $H=0,6$ e $H=0,9$ ) e dos dados i.i.d. . . . . . . . . . . 24

3.2 (a) função seno; (b) amostra com ruído de desvio padrão $\sigma=0,1$; (c) função distribuição $H$; (d) autocorrelograma; (e) estimador proposto (linha cheia) e função original (linha tracejada), limiar a partir do $3^{\circ}$ nível; (f) estimador tratando como se a amostra fosse igualmente espaçada (linha cheia) e função original (linha tracejada), limiar a partir do $3^{\circ}$ nível. . . . . . . . . . . . . . . . . . . . . . . . 26

3.3 (a) função HeaviSine; (b) amostra com ruído de desvio padrão $\sigma=0,1$; (c) estimador proposto (linha cheia) e função original (linha tracejada), limiar a partir do $6^{\circ}$ nível; (d) estimador tratando como se a amostra fosse igualmente espaçada (linha cheia) e função original (linha tracejada), limiar a partir do $6^{\circ}$ nível. . . . . . . . . . . . . .

3.4 (a) função seno transladada no tempo; (b) amostra com ruído de desvio padrão $\sigma=$ 0,05; (c) estimador proposto (linha cheia) e função original (linha tracejada), limiar a partir do $5^{\circ}$ nível; (d) estimador tratando como se a amostra fosse igualmente espaçada (linha cheia) e função original (linha tracejada), limiar a partir do $5^{\circ}$ nível. . . . . . . . 27

3.5 Relação entre renda $R$ e idade $I$ para mulheres brasileiras com mestrado ou doutorado, IBGE, PNAD 2004. A renda é medida pela diferença, em relação à média, do logaritmo natural dos rendimentos do trabalho principal por hora trabalhada. A idade é medida em anos completos até a data da PNAD 2004, com duas casas decimais. 
4.1 Funções seno (a) igualmente espaçada, (b) desigualmente espaçada (linhas tracejadas) e respectivas estimativas via ondaletas (linhas cheias) baseadas em $n=1024$ pontos e $\mathrm{SNR}=7$. Um ruído Gaussiano, seguindo um modelo $\mathrm{AR}(1)$ com coeficiente $\phi=0,7$, foi adicionado à função teste. A ondaleta Symmlet 8 foi usada com aplicação de limiares suaves por níveis a partir do nível $j_{0}=3$.

4.2 Funções HeaviSine (a) igualmente espaçada, (b) desigualmente espaçada (linhas tracejadas) e respectivas estimativas via ondaletas (linhas cheias) baseadas em $n=1024$ pontos e $\mathrm{SNR}=7$. Um ruído Gaussiano, seguindo um modelo $\mathrm{AR}(1)$ com coeficiente $\phi=0,7$, foi adicionado à função teste. A ondaleta Symmlet 8 foi usada com aplicação de limiares suaves por níveis a partir do nível $j_{0}=5 \ldots \ldots \ldots \ldots \ldots$

4.3 Funções Doppler (a) igualmente espaçada, (b) desigualmente espaçada (linhas tracejadas) e respectivas estimativas via ondaletas (linhas cheias) baseadas em $n=1024$ pontos e $\mathrm{SNR}=7$. Um ruído Gaussiano, seguindo um modelo $\mathrm{AR}(1)$ com coeficiente $\phi=0,7$, foi adicionado à função teste. A ondaleta Symmlet 8 foi usada com aplicação de limiares suaves por níveis a partir do nível $j_{0}=8 \ldots \ldots \ldots \ldots \ldots$

4.4 (a) Retornos simples $(R(t))$ de preços de fechamento das ações do Gouverneur Bancorp Inc. e (b) histograma dos dias em que eles ocorreram. De 9 de agosto de 1999 a 6 de

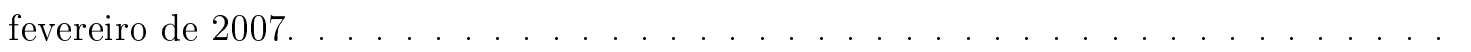

4.5 Gráficos de análise de ondaletas para os retornos simples dos preços de fechamento das ações do Gouverneur Bancorp Inc. da Figura 4.4: (a) Coeficientes empíricos da decomposição de ondaletas, segundo os níveis $j$ e os índices $k$; (b) verificação de correlação dos dados; (c) Coeficientes após aplicação de limiares suaves por níveis; (d)

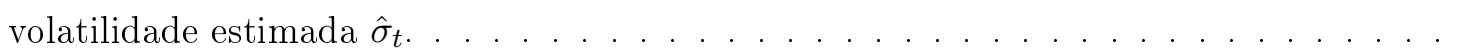

4.6 Retornos simples dos preços de fechamento das ações do Gouverneur Bancorp Inc. (círculos), juntamente com limites de \pm 2 vezes a volatilidade estimada por métodos de ondaletas (linhas) . . . . . . . . . . . . . . . . . . .

5.1 (a) Função seno com ruído i.i.d. $N\left(0,(0,1)^{2}\right)$; (b) mesma função de (a), com ruído $\operatorname{AR}(1)$, coeficiente $\phi=0,8$ e mesma variância $\ldots \ldots \ldots \ldots$

5.2 Amostras da função seno (pontos) com (a) ruído i.i.d. e estimativa linear ; (b) ruído i.i.d. e estimativa não-linear; (c) ruído $\operatorname{AR}(1) \operatorname{com} \phi=0,8$ e estimativa linear; (d) ruído $\operatorname{AR}(1) \operatorname{com} \phi=0,8$ e estimativa não-linear.

5.3 (a) Função salto com ruído i.i.d. $N\left(0,(0,1)^{2}\right)$; (b) mesma função de (a), com ruído $\operatorname{AR}(1)$, coeficiente $\phi=0,8$ e mesma variância 
5.4 Amostras da função salto (pontos) com (a) ruído i.i.d. e estimativa linear ; (b) ruído i.i.d. e estimativa não-linear; (c) ruído $\operatorname{AR}(1) \operatorname{com} \phi=0,8$ e estimativa linear; (d) ruído $\operatorname{AR}(1) \operatorname{com} \phi=0,8$ e estimativa não-linear. . . . . . . . . . . . . . 85

5.5 Gráfico de dispersão da proporção da dívida que foi recuperada $R$ pelo valor da dívida $D$ e traços logo acima do eixo das abscissas ilustrando o espaçamento desigual de $D . \quad$. 87

5.6 (a) Gráfico de dispersão da proporção da dívida que foi recuperada $R$ pelo valor da dívida $D$ (círculos), traços sobre o eixo das ordenadas ilustrando o espaçamento desigual de $D$ e estimativa usando ondaletas deformadas (linha) com limiares por níveis; (b) verificação de autocorrelação; (c) correlograma dos resíduos; (d) gráfico de quantis normais teóricos $q_{0}$ e amostrais $q_{1}$ e reta passando pelos primeiro e terceiro quantis. . .

5.7 (a) Gráfico de dispersão de 256 magnitudes $M$ da cefeida RU Andrômeda pelos dias julianos $t$ entre 2449004 e 2450352 (Janeiro de 1993 a meados de 1996). (b) Histograma da distribuição de 256 dias $t$ de observação da cefeida. . . . . . . . . . . . . .

5.8 (a) Gráfico de dispersão de 256 magnitudes $M$ da cefeida RU Andrômeda pelos dias julianos $t$ entre 2449004 e 2450352 (círculos) e estimativa usando ondaletas deformadas (linha) com limiares por níveis; (b) verificação de autocorrelação; (c) correlograma dos resíduos; (d) gráfico de quantis normais teóricos $q_{0}$ e amostrais $q_{1}$ e reta passando pelos primeiro e terceiro quantis. . . . . . . . . . . . . . . . . 90

5.9 (a) Gráfico de dispersão de 256 magnitudes $M$ da cefeida RU Andrômeda pelos dias julianos $t$ entre 2449004 e 2450352 (cículos) e estimativa usando ondaletas deformadas (linha) com limiar Universal; (b) correlograma dos resíduos; (c) gráfico de quantis normais teóricos $q_{0}$ e amostrais $q_{1}$ e reta passando pelos primeiro e terceiro quantis. . .

6.1 Resultados de simulação para a função densidade Gaussiana, coeficiente autorregressivo igual a 0,8 , tamanho da amostra igual a 256; estimador (linha contínua), função verdadeira (linha tracejada) e estimador \pm 1 erro padrão (linha pontilhada). Métodos (a) iterativo, (b) Universal e (c) SURE. . . . . . . . . . . . . . . . . . .

6.2 Resultados de simulação para a função HeaviSine, coeficiente autorregressivo igual a 0,8, tamanho da amostra igual a 256; estimador (linha contínua), função verdadeira (linha tracejada) e estimador \pm 1 erro padrão (linha pontilhada). Métodos (a) iterativo, (b) Universal e (c) SURE. . . . . . . . . . . . . . . . . . . . . . . . .

6.3 Resultados de simulação para a função Doppler, coeficiente autorregressivo igual a 0,8, tamanho da amostra igual a 256; estimador (linha contínua), função verdadeira (linha tracejada) e estimador \pm 1 erro padrão (linha pontilhada). Métodos (a) iterativo, (b) Universal e (c) SURE. 
6.4 Resultados da simulação para a função Doppler, coeficiente autorregressivo 0,8, três tamanhos de amostra: média do MSE (6.5), com segmentos de linhas representando seu desvio-padrão amostral. . . . . . . . . . . . . . . . . . . . . . 101

6.5 Resultados da simulação para três funções, coeficiente autorregressivo 0,8, tamanho de amostra 256: média do MSE (6.5), com segmentos de linhas representando seu desvio-padrão amostral. . . . . . . . . . . . . . . . . . . . . . . 101

6.6 Resultados da simulação para a função Doppler, tamanho amostral 256, três valores do coeficiente autorregressivo: média do MSE (6.5), com segmentos de linhas representando seu desvio-padrão amostral. . . . . . . . . . . . . . . . . . . . 102

6.7 (a) Série de 2048 dias do índice da NASDAQ. (b) Log-retornos da série em (a). (c) Resíduos quadráticos de um $\operatorname{AR}(2)$ ajustado à série em (b). (d) Função de autocorrelação

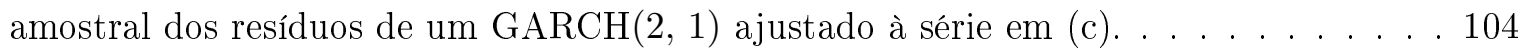

6.8 (a) Ajuste GARCH para os log-retornos quadráticos da Figura 6.7(c). (b) Ajuste aos dados da Figura 6.7(c) obtido com o método iterativo proposto. (c) Volatilidade condicional do modelo $\operatorname{GARCH}(2,1)$ ajustado e curva LOWESS suavizada. (d) Volatilidade condicional do modelo ajustado usando o método iterativo proposto. . . . . . . . . . 105 


\section{Lista de Tabelas}

4.1 MSE do estudo de simulação. As colunas com "\% dif." são as diferenças percentuais entre os MSE do delineamento aleatório e do delineamento igualmente espaçado. Foi atribuído o valor zero quando a diferença era insignificante a $5 \%$, de acordo com testes

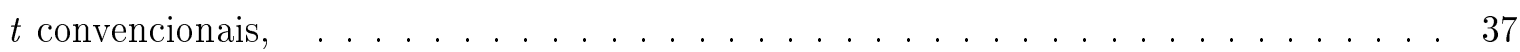

6.1 Simulação de função Gaussiana. . . . . . . . . . . . . . . . . . . . . . . . . 99

6.2 Simulação de função HeaviSine. . . . . . . . . . . . . . . . . . . . . 100

6.3 Simulação de função Doppler. . . . . . . . . . . . . . . . . . . . 100 


\section{Capítulo 1}

\section{Introdução}

Regressão não-paramétrica é uma técnica de recuperação de uma função de regressão a partir de dados observados com erro ou ruído, sem a necessidade de se especificar uma classe paramétrica de funções de regressão. Abordagens tradicionais incluem estimadores de núcleo, análise usando splines, análise de Fourier e, mais recentemente, análise usando ondaletas.

Estas últimas são funções localizadas no tempo (ou no espaço) e na escala, tornando-as apropriadas para analisar funções com descontinuidades (MORETTIN, 1999). Além de regressão não-paramétrica, em Estatística, as ondaletas são usadas em estimação de densidades, estimação do espectro de processos estacionários e estimação do espectro evolutivo de processos não-estacionários, dentre outros. Há uma quantidade muito grande de aplicações às diversas áreas do conhecimento.

O uso de ondaletas em Estatística foi iniciado por Donoho e Johnstone (1994, 1995), que apresentaram um conjunto de procedimentos coerentes, espacialmente adaptativos e ótimos (ou quase ótimos) sobre uma ampla gama de classes de espaços de funções, com diversos graus de suavidade. Esses procedimentos utilizam técnicas de aplicação de limiares ou de encolhimento de ondaletas, que têm como objetivo a redução ou remoção do ruído presente em um sinal, anulando ou diminuindo a magnitude dos coeficientes de ondaletas, respectivamente. A maioria dessas técnicas não exige estimação de parâmetros de suavidade das funções e os estimadores resultantes gozam de boas propriedades estatísticas.

Embora esses procedimentos tenham sido desenvolvidos para estimação na presença de erros não-correlacionados, métodos de ondaletas foram estendidos para a situação onde os erros são correlacionados (JOHNSTONE; SILVERMAN, 1997). Correlação dos resíduos é algo relativamente comum em aplicações práticas de modelos de regressão. Essa correlação pode ter importantes consequências nas propriedades do estimador. Exemplos dessas consequências e uma revisão de métodos para estimação de funções neste contexto, foi feita por Opsomer, Wang e Yang (2001), onde também se discute métodos usando núcleos e splines, além de ondaletas.

A despeito dessas vantagens, esses procedimentos perdem muito de sua simplicidade quando o delineamento é desigualmente espaçado, que ocorre na maioria das aplicações envolvendo regres- 
são não-paramétrica. Para continuar usando os métodos tradicionais de ondaletas, a maioria dos procedimentos aplica algum tratamento prévio para tornar as observações igualmente espaçadas, frequentemente ao preço de alguma perda na precisão do estimador. Para manter a mesma precisão, o preço é um maior tempo de computação ou a restrição a ondaletas com fórmulas analíticas. Uma revisão de diversos desses métodos foi feita por Delouille (2002).

Recentemente surgiram mais duas abordagens para o problema do delineamento desigual. Uma utiliza o conceito de ondaletas adaptativas (DELOUILLE, 2002) que recria uma análise de ondaletas diretamente nos pontos do delineamento. A outra estuda as condições sobre o delineamento em que os procedimentos de ondaletas continuam sendo bons (KERKYACHARIAN; PICARD, 2004).

Especificamente, considere que os pares $\left(Y_{1}, X_{1}\right), \ldots,\left(Y_{n}, X_{n}\right)$ sigam o modelo

$$
Y_{i}=f\left(X_{i}\right)+\epsilon_{i} \quad i=1,2, \ldots, n,
$$

onde $Y_{i}$ é uma variável aleatória, $X_{i}$ pode ser uma variável aleatória ou um valor fixo, $f$ é uma função desconhecida a ser estimada e a sequência $\left\{\epsilon_{i}\right\}_{i=1}^{n}$ é gerada por um processo estocástico de média zero, correlacionado ou não.

Em geral, o objetivo é estimar $f$ globalmente por um estimador $\hat{f}$ com menor risco de norma $L_{p}$ (considerando delineamento fixo):

$$
R_{p}(\hat{f}, f)=E\left(\int(\hat{f}(x)-f(x))^{p} d x\right)
$$

onde a integral deve estar bem definida e, para que o risco faça sentido, deve-se assumir certas condições de regularidade em relação à função $f$ como, por exemplo, que $f$ pertença a alguma classe de funções como Hölder, Sobolev, Besov etc.

Estimadores que minimizam o risco máximo dentro de uma classe de funções são chamados estimadores minimax. O risco minimax associado tem sido largamente utilizado para avaliar o desempenho de um estimador de uma função que se assume pertencer a uma específica classe de funções (OPSOMER; WANG; YANG, 2001). Devido à dificuldade em se calcular esse risco, em geral sua taxa de convergência a zero, como função do tamanho $n$ da amostra, é frequentemente considerada. Um estimador com risco convergindo à taxa minimax uniformemente sobre uma classe de funções é dito minimax-ótimo para tal classe.

Nesta tese, o interesse é obter tais taxas para os estimadores propostos quando há erros correlacionados. Essas taxas são apresentadas nos Capítulos 3, 4 e 5, onde quatro métodos de regressão não-paramétrica via ondaletas, com delineamento desigualmente espaçado, são estudados na presença de erros correlacionados, oriundos de processos estocásticos. Em cada um desses capítulos também são apresentadas condições sobre os erros e adaptações aos procedimentos, necessárias à obtenção de taxas de convergência quase minimax para os estimadores. Também é desenvolvido e proposto um 
procedimento iterativo semi-paramétrico geral, facilmente adaptável a qualquer um dos quatro métodos de ondaletas estudados, quando há erros em séries temporais. Esse procedimento, desenvolvido no Capítulo 6, ainda é adaptável a outros métodos de ondaletas não estudados nesta tese e também inclui os casos de estimação não-paramétrica de funções de regressão.

De modo específico, inicialmente generaliza-se a metodologia de Cai e Brown (1998), para estimação via ondaletas em amostras desigualmente espaçadas, mas na presença de erros estacionários Gaussianos correlacionados. Essa generalização permite incluir os casos específicos em que sinais são amostrados de modo desigualmente espaçado e de regressões com delineamento fixo. Mostra-se que se a função verdadeira for membro de uma classe de Hölder por partes, então o procedimento é quase ótimo. Mesmo para erros com memória longa, a taxa de convergência do procedimento é quase minimax relativamente ao caso de erros i.i.d. Como o procedimento VisuShrink é um caso especial dessa metodologia, esses resultados permanecem válidos para ele.

Em seguida apresentam-se alguns resultados em regressão não-paramétrica usando métodos de ondaletas na presença de erros gaussianos estacionários autocorrelacionados, e quando a variável explicativa segue uma distribuição Uniforme ou provém de uma amostragem estocástica como a jittered. Técnicas de amostragem estocástica são de interesse porque podem reduzir certos problemas de aliasing associados com amostragem em uma grade regular (DIPPÉ; WOLD, 1985) e os resultados apresentados generalizam os de Cai e Brown (1999). Nesses casos especiais, mostra-se que as amostras podem ser tratadas como se fossem igualmente espaçadas e com ruído correlacionado; i.e., o estimador alcança uma taxa de convergência quase ótima.

Uma tentativa de generalização desses resultados a fim de incluir outros delineamentos, é discutida em seguida sob o conceito de ondaletas deformadas. A abordagem de ondaletas deformadas tenta responder para quais classes de delineamentos é ótima a aplicação dos métodos usuais de ondaletas, ignorando a irregularidade do delineamento. Sob condições bastante gerais, mostra-se que essa abordagem é assintoticamente quase ótima.

Resultados quase ótimos do ponto de vista minimax e de risco ideal também são obtidos, sob condições bastante gerais do delineamento e do processo estocástico dos erros, para os procedimentos que se utilizam de ondaletas adaptativas de Haar e usando esquemas de lifting.

Todas essas abordagens baseiam-se na aplicação de limiares aos coeficientes de ondaletas, com diferentes limiares em cada nível de resolução. A obtenção desses limiares pode ser problemática para níveis com poucos coeficientes e, por isso, apresenta-se uma alternativa onde a autocorrelação é tratada de modo paramétrico, permitindo que os métodos de ondaletas sejam usados apenas para a estimação da função. Constitui-se em um método iterativo semi-paramétrico que toma emprestado algumas ideias do procedimento de Cochrane e Orcutt (1949). Os resultados de simulação mostram que o método proposto é pelo menos tão bom quanto os melhores métodos usados com ondaletas, independentemente do tipo de autocorrelação presente nos erros. 
Portanto, esta tese está organizada da seguinte forma. No Capítulo 2 é feita uma revisão de conceitos e resultados necessários ao desenvolvimento da tese. No Capítulo 3, a metodologia de Cai e Brown (1998), para regressão não-paramétrica com delineamento fixo, é generalizada para incluir casos em que os erros são correlacionados. No Capítulo 4, são estudados dois tipos de delineamentos aleatórios em que é possível utilizar os procedimentos usuais de ondaletas, na presença de erros correlacionados gaussianos estacionários. No Capítulo 5 são estudadas condições impostas à correlação dos erros e ao delineamento para que os procedimentos usuais de ondaletas possam ser utilizados. Também é estudado o comportamento das ondaletas adaptativas na presença de erros correlacionados. O procedimento semi-paramétrico é proposto no Capítulo 6. Finalmente, algumas conclusões e indicações de pesquisas futuras são dadas no Capítulo 7 . 


\section{Capítulo 2}

\section{Preliminares}

Neste capítulo são apresentados alguns conceitos, resultados e métodos relacionados com a análise de ondaletas clássicas, deformadas e adaptativas. Sobre essa fundamentação básica, se apoiarão as demonstrações dos resultados obtidos nos Capítulos 3 a 6 .

\subsection{Métodos Usando Ondaletas}

A análise de Fourier e a análise usando splines ou ondaletas se utilizam da expansão ou da aproximação de certas funções usando senos e co-senos, polinômios e ondaletas. Estas últimas são funções localizadas no tempo (ou no espaço) e na escala, tornando-as apropriadas para analisar funções com descontinuidades.

Uma base $\left\{\phi_{j, k}(t) \cup \psi_{j, k}(t), j, k \in \mathbb{Z}\right\}$ do espaço $L_{2}(\mathbb{R})$, formada de ondaletas, pode ser gerada por meio de uma dilatação binária e de uma translação diádica de uma ondaleta-pai $\phi$ (ou função-escala) e de uma ondaleta-mãe $\psi$ :

$$
\phi_{j, k}(t)=2^{j / 2} \phi\left(2^{j} t-k\right), \quad \psi_{j, k}(t)=2^{j / 2} \psi\left(2^{j} t-k\right), j, k \in \mathbb{Z}
$$

A ortonormalidade dessa base depende da escolha das funções $\phi$ e $\psi$. Se $\psi$ (ou $\phi$ ) tiver suporte compacto em $[0, N]$, então $\psi_{j, k}($ ou $\phi)$ terá suporte em $\left[2^{-j} k, 2^{-j}(N+k)\right]$.

A função $\phi$ deve satisfazer $\int \phi=1$ e a função $\psi$ deve ser tal que $\int \psi=0$ (admissível) e $\int|\psi|<\infty$. Uma ondaleta é dita $r$-regular se possui $r$ momentos nulos e $r$ derivadas contínuas.

Exemplos de ondaletas são as ondaletas de Morlet, de Shannon e o Chapéu Mexicano, que têm expressões analíticas. As Daublets e as Symmlets (DAUBECHIES, 1992) não têm expressões analíticas, mas são ondaletas de suporte compacto muito usadas na prática.

Uma base de ondaletas tem associada uma análise de multirresolução em $L_{2}(\mathbb{R})$ que permite que se analise os dados através de distintos níveis de resolução, adicionando ou desprezando detalhes. Mais formalmente, sejam $V_{j}$ e $W_{j}$ os subespaços fechados gerados por $\phi_{j, k}, k=0, \ldots, 2^{j}-1 \mathrm{e}$ $\psi_{j, k}, k=0, \ldots, 2^{j}-1$, respectivamente, então a associada análise de multirresolução satisfaz: 
1. $\cdots \subset V_{-1} \subset V_{0} \subset V_{1} \subset \cdots$

2. $\overline{\cup_{j} V_{j}}=L_{2}(\mathbb{R})$;

3. $\cap_{j} V_{j}=\{0\}$;

4. $f(t) \in V_{j} \Leftrightarrow f(2 t) \in V_{j+1}, \forall j$;

5. $V_{j+1}=V_{j} \oplus W_{j}, \quad W_{j} \perp V_{j}$.

Assim, para um dado nível de resolução $j_{0}$ inicial, uma função $f \in L_{2}(\mathbb{R})$ pode ser expandida em uma série de ondaletas (em sentido de norma $L_{2}$ ):

$$
f(x)=\sum_{k=0}^{2^{j_{0}}-1} c_{j_{0}, k} \phi_{j_{0}, k}(x)+\sum_{j=j_{0}}^{\infty} \sum_{k=0}^{2^{j}-1} d_{j, k} \psi_{j, k}(x),
$$

onde

$$
c_{j_{0}, k}=\left\langle f, \phi_{j_{0}, k}\right\rangle, \quad d_{j, k}=\left\langle f, \psi_{j, k}\right\rangle
$$

Essa transformada de ondaletas decompõe uma função em componentes com diferentes resoluções. Os coeficientes do nível mais grosso são os $c_{j_{0}, k}$, representando a estrutura grossa da função $f$. Os coeficientes de detalhes são denotados por $d_{j, k}$ e representam as estruturas cada vez mais finas em $f$, à medida em que o nível de resolução $j$ aumenta.

Similarmente, pode-se denotar as ondaletas periodizadas por

$$
\phi_{j, k}^{p}(t)=\sum_{l \in \mathbb{Z}} \phi_{j, k}(t-l), \quad \psi_{j, k}^{p}(t)=\sum_{l \in \mathbb{Z}} \psi_{j, k}(t-l),
$$

para $t \in[0,1]$. Se as ondaletas originais forem ortonormais, então a coleção

$$
\phi_{j_{0}, k}^{p}, k=0, \ldots, 2^{j_{0}-1} ; \quad \psi_{j, k}^{p}, j \geq j_{0}, k=0, \ldots, 2^{j}-1
$$

constitui uma base ortonormal de $L_{2}[0,1]$, para algum nível inicial $j_{0}$. Estas serão as ondaletas usadas quando o delineamento estiver em $[0,1]$ e, portanto,o sobrescrito " $p$ " será suprimido nesses casos.

Ondaletas caracterizam completamente muitos espaços tradicionais de funções como os de Hölder, Sobolev e de Besov. Um espaço de funções que será muito usado nesta tese é caracterizado pela

Definição 1 Para qualquer número real $\alpha>0$, a classe de Hölder por partes $\Lambda^{\alpha}(M, B, m)$ em $[0,1]$, com até $m$ pontos de descontinuidade, consiste das funçôes $f$ satisfazendo:

1. $|f| \leq B$; 
2. existem $l \leq m$ pontos $0 \leq a_{1} \leq \cdots \leq a_{l} \leq 1$ tais que, para todos $a_{i} \leq x, y<a_{i+1}, i=0,1, \ldots, l$ com $a_{0}=0$ and $a_{l+1}=1$,

(a) $|f(x)-f(y)| \leq M|x-y|^{\alpha}$ se $0<\alpha \leq 1$;

(b) $\left|f^{(\lfloor\alpha\rfloor)}(x)-f^{(\lfloor\alpha\rfloor)}(y)\right| \leq M|x-y|^{\alpha-\lfloor\alpha\rfloor} e\left|f^{(1)}(x)\right| \leq B$ se $\alpha>1$.

Em aplicações, são amostrados dados da função $f$ e, à uma base ortonormal de ondaletas, está geralmente associada uma transformada discreta de ondaletas exata, que transforma os dados amostrados para o domínio dos coeficientes de ondaletas. Nos métodos usuais que usam ondaletas, os dados são observações das variáveis aleatórias $Y_{1}, \ldots, Y_{n}$, onde $Y_{i}=f(i / n)+e_{i}$, a função $f$ é desconhecida e os erros (ou ruídos) $e_{i}$ possuem média zero e variância $\sigma^{2}<\infty$.

Usando uma transformada discreta, os dados são transformados em coeficientes (empíricos) de ondaletas e um estimador da função $f$ é obtido por meio da transformada inversa dos coeficientes, após a redução ou remoção do ruído. A função $f$ é desconhecida e pode-se estar interessado em estimá-la globalmente com menor IMSE

$$
R_{2}(\hat{f}, f)=E\|\hat{f}-f\|_{2}^{2}=\int_{0}^{1} E(\hat{f}(x)-f(x))^{2} d x
$$

onde o estimador $\hat{f}$ é dado de forma genérica por

$$
\hat{f}(x)=\sum_{k=0}^{2^{j_{0}}-1} \hat{c}_{j_{0}, k} \phi_{j_{0}, k}(x)+\sum_{j=j_{0}}^{J} \sum_{k=0}^{2^{j}-1} \hat{d}_{j, k} \psi_{j, k}(x),
$$

seguindo a equação (2.1). Estimativas dos coeficientes são geralmente obtidas aplicando-se limiares aos coeficientes empíricos que, por sua vez, são obtidos diferentemente para cada um dos métodos descritos nesta tese.

O método mais usado com ondaletas, para se obter o estimador $\hat{f}$, é o procedimento VisuShrink (DONOHO; JOHNSTONE, 1994), descrito a seguir.

Denote pela matriz $W$ uma transformada discreta de ondaletas que é aplicada a $Y=\left(Y_{1}, \ldots, Y_{n}\right)^{T}$, onde $Y=f+e, f=(f(1 / n), \ldots,(n / n))^{T}, e=\left(e_{1}, \ldots, e_{n}\right)^{T}$ e $n=2^{J}$, com $J \in \mathbb{N}$. Tome $j_{0}=0$ e escreva

$$
d_{j, k}=(W Y)_{j, k}, \quad j=0,1, \ldots, J-1, \quad k=0, \ldots, 2^{j}-1,
$$

onde $d_{-1,0} \equiv c_{0,0}$. Sejam $\theta=W f$ a transformada de ondaletas da função $f$, e $z=W e$ a transformada de ondaletas do ruído.

Para a construção do estimador, defina a função Limiar Duro

$$
\eta_{H}(d, \lambda)=d I(|d| \geq \lambda)
$$


O limiar $\lambda$ é comumente escolhido como sendo $\lambda=\sigma \sqrt{n^{-1} 2 \log n}$ quando os erros $e_{i}$ são variáveis aleatórias i.i.d. $N\left(0, \sigma^{2}\right)$, com $\sigma^{2}$ conhecido. Também pode-se definir a função Limiar Suave

$$
\eta_{S}(d, \lambda)=\operatorname{sgn}(d)(|d|-\lambda)
$$

que geralmente fornece estimadores com melhor apresentação visual do que usando Limiar Duro.

O estimador VisuShrink (DONOHO; JOHNSTONE, 1994) é construído aplicando-se limiares aos coeficientes de ondaletas (2.3) com limiar $\lambda=\sigma \sqrt{n^{-1} 2 \log n}$ e, então, aplicando a transformada inversa de ondaletas. Usando a função Limiar Suave, definimos $\hat{\theta}$ por

$$
\begin{gathered}
\hat{\theta}=\left(c_{0,0}, \hat{d}_{0,0}, \hat{d}_{1,0}, \ldots, \hat{d}_{J-1,2^{J-1}-1}\right)^{T}, \\
\hat{d}_{j, k}=\eta_{S}\left(d_{j, k}, \lambda\right)
\end{gathered}
$$

e o estimador $\hat{f}$ por

$$
\hat{f}=W^{T} \hat{\theta} .
$$

Se os erros $\epsilon_{i}$ forem correlacionados, gaussianos de média zero e estacionários, então pode-se mostrar (JOHNSTONE; SILVERMAN, 1997; FLANDRIN, 1994) que se a transformada discreta de ondaletas for invariante no tempo então, em cada nível, a distribuição dos elementos $z_{j, k}$ de $z$ será estacionária e a variância de $z_{j, k}$ dependerá apenas do nível $j$ :

$$
\sigma_{j}^{2}=\operatorname{Var}\left(z_{j, k}\right)
$$

para cada $j=0, \ldots, J-1$.

Assim, uma extensão natural do método VisuShrink é a aplicação de limiares por níveis, aos coeficientes empíricos de ondaletas. Para isso, seja $\left\{\lambda_{j}=\sigma_{j} \sqrt{n^{-1} 2 \log n}\right\}_{j=0}^{J-1}$ uma sequência de limiares a serem aplicados aos coeficientes dos respectíveis níveis $j$ tal que

$$
\hat{\theta}_{j, k}=\eta_{S}\left(d_{j, k}, \lambda_{j}\right)
$$

e defina $\hat{\theta}$ como sendo o correspondente estimador de $\theta$ formado pelos $\hat{d}_{j, k}$, a ser usado em (2.5). Pode-se mostrar (DONOHO; JOHNSTONE, 1994; HÄRDLE et al., 1998) que $\hat{f}$ é quase minimax-ótimo para uma ampla classe de funções.

Na prática a transformada $W$ e sua inversa $W^{T}$ são realizadas por um rápido algoritmo de ordem $O(n)$ (DONOHO; JOHnstone, 1995, Seção 2.1). A aplicação dos limiares é restrita a níveis $j$ maiores que um nível de resolução inicial $j_{0}$ especificado pelo usuário, abaixo do qual supõe-se que o sinal predomine sobre o ruído. Nos níveis de detalhes maiores que $j_{0}$, geralmente há uma considerável quantidade de coeficientes em cada nível e a representação da função $f$ pode ser assumida como sendo esparsa. Além disso, a variância $\sigma^{2}$ do ruído é geralmente estimada a partir dos dados, usualmente 
através do uso de estimadores robustos como o MAD(DONOHO; JOHNSTONE, 1994; JOHNSTONE; SILVERMAN, 1997):

$$
\hat{\sigma}=\operatorname{med}\left\{d_{J-1, k} ; k=0, \ldots, 2^{J}-1\right\} / 0,6745 .
$$

Em parte, a escolha de $\lambda=\sigma \sqrt{n^{-1} 2 \log n}$ é atrativa por sua simplicidade e pelo fato de que, se $Z_{1}, \ldots, Z_{n}$ forem variáveis aleatórias normalmente distribuídas com média 0 e variância $\sigma_{i}^{2}$, então

$$
P\left(\max _{1 \leq i \leq n}\left|Z_{i} / \sigma_{i}\right|>\sqrt{2 \log n}\right) \rightarrow 0
$$

quando $n \rightarrow \infty$, sendo as variáveis independentes ou não (JOHNSTONE; SILVERMAN, 1997). Esta propriedade de "consistência" será útil para o desenvolvimento da proposta do Capítulo 6.

\subsection{Delineamento Uniforme}

A fim de relaxarmos um pouco a necessidade de amostras igualmente espaçadas de $f$, considere o modelo

$$
Y\left(X_{i}\right)=f\left(X_{i}\right)+\epsilon_{i}
$$

para $i=1, \ldots, n, n=2^{J}, J \in \mathbb{N}$, onde $X_{i}$ são variáveis aleatórias i.i.d. Uniforme $[0,1]$ e os $\epsilon_{i}$ são variáveis i.i.d. $N\left(0, \sigma^{2}\right) \operatorname{com} \sigma^{2}<\infty$ conhecido e independentes de $X_{i}$.

Sejam $0 \leq X_{(1)}<X_{(2)}<\cdots<X_{(n)} \leq 1$ as estatísticas de ordem dos $X_{i}$. Substituindo os rótulos de acordo com a ordem dos $X_{i}$, esse modelo pode ser reescrito como

$$
Y_{i}=f\left(X_{(i)}\right)+e_{i} \quad i=1, \ldots, n,
$$

onde $Y_{i} \equiv Y\left(X_{(i)}\right)$ e $e_{i}=\epsilon_{j} \Leftrightarrow X_{(i)}=X_{j}, i=1, \ldots, n$. Os dados consistem de observações dos pares de variáveis aleatórias $\left(X_{(1)}, Y_{1}\right),\left(X_{(2)}, Y_{2}\right), \ldots,\left(X_{(n)}, Y_{n}\right)$. Como $X_{i}$ é uniformemente distribuída em $[0,1]$, então $X_{(i)}$ possui densidade $\operatorname{Beta}(i, n-i+1)$ e $E\left(X_{(i)}\right)=i /(n+1)$, para $i=1, \ldots, n$, com $n$ fixo (CAI; BROWN, 1999). Assim, de modo aproximado, tem-se um delineamento amostral igualmente espaçado:

$$
\left(\frac{1}{n+1}, y_{1}\right),\left(\frac{2}{n+1}, y_{2}\right), \ldots,\left(\frac{n}{n+1}, y_{n}\right)
$$

e o procedimento VisuShrink é aplicável diretamente aos dados.

Pode-se mostrar (CAI; BROWN, 1999) que o estimador obtido com a aplicação do procedimento VisuShrink diretamente aos dados, alcança a taxa ótima de convergência a menos de um fator logarítmico em classes de Hölder $\Lambda^{\alpha}(M, B, 0)$, com $1 / 2 \leq \alpha \leq r$. Este resultado é verdadeiro para limiares duros ou suaves. Isso mostra que, no caso de delineamento uniforme e erros normais independentes, os dados podem ser tratados como se fossem igualmente espaçados. Um argumento isométrico também pode ser usado para justificar essa prática para outros tipos de amostragem não uniforme (SARDY et al., 1999). 


\subsection{Ondaletas Deformadas}

Além do argumento isométrico (SARDY et al., 1999) usado para justificar a aplicação do procedimento VisuShrink diretamente a dados desigualmente espaçados, pode-se usar o conceito de ondaletas deformadas (KERKYACHARIAN; PICARD, 2004; CLERC; MALlat, 2003; Le Pennec; MALlat, 2003). Para tanto, considere o modelo

$$
Y\left(X_{i}\right)=f\left(X_{i}\right)+\epsilon_{i}
$$

para $i=1, \ldots, n, n=2^{J}, J \in \mathbb{N}$, onde $X_{i}$ são variáveis aleatórias i.i.d. com distribuição $G$ conhecida e respectiva densidade $g$, com suporte compacto em $[a, b]$. Além disso, suponha que $\epsilon_{i}$ sejam variáveis i.i.d., independentes de $X_{i}$, seguindo alguma densidade com média zero e variância $\sigma^{2}<\infty$ conhecida.

Sejam $a \leq X_{(1)}<X_{(2)}<\cdots<X_{(n)} \leq b$ as estatísticas de ordem dos $X_{i}$. Substituindo os rótulos de acordo com a ordem dos $X_{i}$ 's, esse modelo pode ser reescrito como

$$
Y_{i}=f\left(X_{(i)}\right)+e_{i} \quad i=1, \ldots, n,
$$

onde $Y_{i} \equiv Y\left(X_{(i)}\right)$ e $e_{i}=\epsilon_{j} \Leftrightarrow X_{(i)}=X_{j}, i=1, \ldots, n$. Os dados consistem de observações dos pares de variáveis aleatórias $\left(X_{(1)}, Y_{1}\right),\left(X_{(2)}, Y_{2}\right), \ldots,\left(X_{(n)}, Y_{n}\right)$. Se $X_{(i)}$ for uma boa aproximação para $G^{-1}(i / n)$, então tem-se aproximadamente o modelo

$$
Y_{i}=f\left(G^{-1}(i / n)\right)+e_{i} \quad i=1, \ldots, n .
$$

Assim, expandindo a função $f\left(G^{-1}(y)\right) \equiv f \circ G^{-1}(y)$ em uma base ortonormal de ondaletas $\left\{\phi_{j_{0}, k}(t) \cup \psi_{j, k}(t), j \geq j_{0}, k \in \mathbb{Z}\right\}$, os coeficientes aproximam, respectivamente, aqueles da expansão da função $f$, em uma base de ondaletas deformadas por $G$, i.e.:

$$
\begin{aligned}
d_{j, k} & =\left\langle f \circ G^{-1}, \psi_{j, k}\right\rangle \\
& =\int_{0}^{1} \psi_{j, k}(y) f\left(G^{-1}(y)\right) d y \\
& =\int_{a}^{b} \psi_{j, k}(G(x)) f(x) g(x) d x \\
& =E\left(\psi_{j, k}\left(G\left(X_{1}\right)\right) f\left(X_{1}\right)\right) \\
& =n^{-1} \sum_{i=1}^{n} E\left(\psi_{j, k}\left(G\left(X_{i}\right)\right) f\left(X_{i}\right)\right) \\
& \approx E\left(\int_{a}^{b} \psi_{j, k}(G(x)) f(x) d x\right)=E\left(\left\langle f, \psi_{j, k} \circ G\right\rangle\right) .
\end{aligned}
$$

A nova base formada pelas ondaletas deformadas não é mais ortonormal mas é computacionalmente simples, permitindo que se utilize os mesmos métodos já desenvolvidos. Nesse sentido, a especificação das condições necessárias à distribuição $G$ para que essa aproximação seja satisfatória, 
generalizam os resultados da Seção 2.2. Para especificação dessas condições sobre $G$, são necessárias algumas definições (KERKYACHARIAN; PICARD, 2004).

Definição 2 Se $\mathcal{B}$ é o conjunto de todos os intervalos de $\mathbb{R}$ e se $\omega$ é uma função mensurável, então a função maximal de Hardy-Littlewood associada a $\omega$ é

$$
\omega^{*}(x)=\sup _{I \in \mathcal{B}, x \in I}\left(\frac{1}{|I|} \int_{I} \omega(u) d u\right)
$$

Definição 3 Para $1<p<\infty, 1 / p+1 / q=1$, uma função mensurável $\omega \geq 0$ é um peso de Muckenhoupt (pertence à classe $A_{p}$ de Muckenhoupt) se existe $0<C<\infty$ tal que para qualquer intervalo $I \subset \mathbb{R}$,

$$
\left(\frac{1}{|I|} \int_{I} \omega(x) d x\right)^{1 / p}\left(\frac{1}{|I|} \int_{I} \omega(x)^{-q / p} d x\right)^{1 / q} \leq C
$$

Para $p=1, \omega \geq 0$ pertence à classe $A_{1}$ de Muckenhoupt se existe $0<C<\infty$ tal que

$$
\omega^{*} \leq C \omega(x) \quad \text { q.c. }
$$

onde $\omega^{*}$ é a função maximal de Hardy-Littlewood. Para $p=\infty$, definimos

$$
A_{\infty}=\bigcup_{p \geq 1} A_{p}
$$

Os espaços de Muckenhoupt são crescentes em $p$ e englobam diversas funções; por exemplo, uma função limitada inferior e superiormente, pertence a $A_{p}$, para todo $p \geq 1$. A definição de peso de Muckenhoupt de certa forma quantifica quão próximo $\omega$ está de um peso uniforme, onde a função e sua inversa ponderam igualmente cada intervalo.

A seguinte condição é suficiente (KERKYACHARIAN; PICARD, 2004) para que a expansão da função $f$, em uma base de ondaletas deformadas por $G$, seja bem aproximada pela expansão de $f \circ G^{-1}$, na base de ondaletas não deformadas correspondente:

$$
y \mapsto \omega(y)=\frac{1}{g\left(G^{-1}(y)\right)} \in A_{p}([a, b]) .
$$

Isso, de certa forma quantifica a suposição usual de que o delineamento atribua densidade suficiente a qualquer intervalo do suporte da função $f$. Tipicamente, se $G$ for definida em $(a, b)$ e se $G^{-1}$ for localmente Lipschitz em $(0,1)$, então, $\omega \in A_{p} \operatorname{com} 1<p<\infty$ e

$$
G^{-1}(G(x))=x \text { q.c., } \quad G\left(G^{-1}(y)\right)=y \text { q.c. e } \quad \int_{\mathbb{R}} h(G(x)) d x=\int_{\mathbb{R}} h(y) \omega(y) d y,
$$

para qualquer função mensurável $h$ não negativa (KERKYACHARIAN; PICARD, 2004).

Para muitas aplicações estatísticas, especialmente do ponto de vista assintótico, uma base de 
ondaletas deformadas $\left\{\phi_{j_{0}, k}(G(t)) \cup \psi_{j, k}(G(t)), j \geq j_{0}, k \in \mathbb{Z}\right\}$ gera uma aproximação satisfatória se satisfizer às seguintes duas propriedades:

Definição 4 Uma base $\left\{\zeta_{i}, i \in \mathbb{N}\right\}$ possui a propriedade de encolhimento (ou incondicional) se existe uma constante $C>0$ tal que

$$
\left\|\sum_{i \in \mathbb{N}} d_{i} \zeta_{i}\right\|_{p} \leq C\left\|\sum_{i \in \mathbb{N}} d_{i}^{\prime} \zeta_{i}\right\|_{p}
$$

se $\left|d_{i}\right| \leq\left|d_{i}^{\prime}\right|$

Definição 5 Uma base $\left\{\zeta_{i}, i \in \mathbb{N}\right\}$ possui a propriedade $p$-Temlyakov se existem constantes $c_{p}$ e $C_{p}$ tais que

$$
c_{p} \int \sum_{i \in F}\left|\zeta_{i}\right|^{p} \leq \int\left(\sum_{i \in F}\left|\zeta_{i}\right|^{2}\right)^{p / 2} \leq C_{p} \int \sum_{i \in F}\left|\zeta_{i}\right|^{p},
$$

para qualquer conjunto finito $F$ de inteiros. Em uma base ortogonal $c_{p}=C_{p}$.

Essas propriedades são verdadeiras para $p=2$ quando $\zeta_{i}$ é uma base ortonormal, permitindo uma extensão para $p>2$. Também são verdadeiras para bases de ondaletas com suporte compacto mas em geral são falsas para bases deformadas, mesmo para $p=2$. Entretanto, existe o seguinte (KERKYACHARIAN; PICARD, 2002, 2006) teorema.

Teorema 1 Sob as condiçôes (2.9), a base de ondaletas deformadas $\left\{\phi_{j, k}(G(\cdot)) \cup \psi_{j, k}(G(\cdot)), j \geq\right.$ $\left.j_{0}, k \in \mathbb{Z}\right\}$ satisfaz as propriedades de encolhimento e p-Temlyakov.

Além dessas propriedades, assim como as ondaletas no caso igualmente espaçado, as ondaletas deformadas possuem também as seguites propriedades:

$$
\begin{aligned}
& E\left(\psi_{j, k}(G(X))\right)=\int_{a}^{b} \psi_{j, k}(G(x)) g(x) d x=\int_{0}^{1} \psi_{j, k}(y) d y=0 ; \\
& E\left(\psi_{j, k}^{2}(G(X))\right)=\int_{a}^{b} \psi_{j, k}^{2}(G(x)) g(x) d x=\int_{0}^{1} \psi_{j, k}^{2}(y) d y=1 .
\end{aligned}
$$

Como geralmente na prática a distribuição $G$ não é conhecida, usa-se a distribuição empírica e isto é equivalente a se realizar uma estimação usando ondaletas pelo procedimento VisuShrink, com Limiar Duro, tratando os dados como se fossem igualmente espaçados. Pode-se mostrar que, quando o delineamento satisfaz à condição (2.8), o estimador $\hat{f}$ obtido é quase minimax-ótimo, sobre uma ampla classe de funções (KERKYACHARIAN; PICARD, 2004).

\subsection{Ondaletas Adaptativas e Lifting}

Em vez de se interpolar ou de se estudar as condições em que se pode realizar estimação usando ondaletas como se os dados fossem igualmente espaçados, pode-se alterar o algoritmo que gera a 
transformada, por meio de ondaletas adaptativas ao delineamento. Para isso, considere o modelo de regressão

$$
Y_{t}=f\left(X_{t}\right)+\epsilon_{t}, \quad t=1, \ldots, n \quad n=2^{J} \quad J \in \mathbb{N},
$$

onde $X_{t}$ é uma variável aleatória com função distribuição $G$ conhecida e respectiva densidade $g, f$ é uma função desconhecida em $\Lambda^{\beta}(L, B, 0)$ a ser estimada com baixo risco e $\epsilon_{t}$ é um termo de erro i.i.d. $N\left(0, \sigma_{\epsilon}^{2}\right)$, com $\sigma_{\epsilon}^{2}<\infty$. As variáveis $X_{t}$ são independentes e $\epsilon_{t}$ é independente de $X_{t}$, para todo $t$.

Seja $L_{2}(d G) \equiv L_{2}(I, \Sigma, \mu)$ um espaço ponderado onde $I \subset \mathbb{R}, \Sigma=\sigma(I)$ e $\mu=d G$ é a medida de Borel-Stieltjes $\sigma$-finita em $\Sigma$ associada a $G$. Também seja $G^{-1}(u)=\inf \{x: G(x) \geq u\}$ e denote por $\hat{G}$ a distribuição empírica de $G$ com uma medida discreta $\hat{\mu}_{n}=d \hat{G}$ associada.

A abordagem em ondaletas adaptativas é decompor uma função $f(x)$ em uma base de ondaletas $\left\{\check{\phi}_{j_{0}, k}(t) \cup \check{\psi}_{j, k}(t), j=j_{0}, \ldots, J, j_{0} \in\{1, \ldots, J\}, k \in \mathcal{K}_{j}\right\}$ que é ortogonal no espaço $L_{2}(d G)$ :

$$
f(x)=\sum_{k \in \mathcal{K}_{j_{0}}} s_{j_{0}, k} \check{\phi}_{j_{0}, k}(x)+\sum_{j=j_{0}}^{J} \sum_{k \in \mathcal{K}_{j}} d_{j, k} \check{\psi}_{j, k}(x),
$$

onde $d_{j, k}=\left\langle f, \psi_{j, k}\right\rangle_{d G}=\int f(x) \psi_{j, k}(x) d G(x)$ e $\mathcal{K}_{j}$ é um conjunto de índices associado ao nível $j$. Para construir tal base, seja

$$
I_{j, k}^{\circ}=\left[G^{-1}\left(\frac{k-1}{2^{j}}\right), G^{-1}\left(\frac{k}{2^{j}}\right)\right),
$$

onde $j=1, \ldots, \mathrm{J}, k=1, \ldots, 2^{j}$, com $J$ denotando a escala mais fina considerada na decomposição. Neste esquema, a medida de um intervalo $I_{j, k}^{\circ}$ é simplesmente igual a $2^{-j}$, e os pontos são igualmente espaçados em $L_{2}(d G)$. Para uma dada realização $X_{1}, \ldots, X_{n}$, defina

$$
I_{j, k}=\left[X_{\left((k-1) n_{j}+1\right)}, X_{\left(k n_{j}+1\right)}\right)
$$

onde $k=1, \ldots, 2^{j}, n_{j}=n 2^{-j}, j_{0} \leq j \leq J$ para algum nível inicial $j_{0}$, e denote por $X_{(1)} \leq \cdots \leq X_{(n)}$ as estatísticas de ordem da variável do delineamento. Como convenção, seja $X_{(n+1)}=X_{(n)}+\left(X_{(n)}-\right.$ $\left.X_{(1)}\right) / n$. Além disso, nesta seção e sempre que se tratar de ondaletas adaptativas, os índices $j$ e $k$ iniciarão em 1, ao invés de zero, mantendo, desse modo, a notação utilizada em Delouille (2002).

Assim, uma base ortogonal em $L_{2}(d G)$ é formada pelas funções escala não balanceadas de Haar (GIRARDI; SWELDENS, 1997)

$$
\check{\phi}_{j, k}(x)=\frac{1}{\mu\left(I_{j, k}\right)^{1 / 2}} I\left(x \in I_{j, k}\right),
$$


e as correspondentes ondaletas

$$
\check{\psi}_{j, m}(x)=\frac{1}{\mu\left(I_{j, k}\right)^{1 / 2}}\left(\sqrt{\frac{\mu\left(I_{j+1, l}\right)}{\mu\left(I_{j+1, l+1}\right)}} I\left(x \in I_{j+1, l+1}\right)-\sqrt{\frac{\mu\left(I_{j+1, l+1}\right)}{\mu\left(I_{j+1, l}\right)}} I\left(x \in I_{j+1, l}\right)\right),
$$

onde o índice $m$ é usado para denotar a localização dos intervalos $I_{j, m}$ tal que $I_{j, m}=I_{j+1, l} \cup I_{j+1, l+1}$ para algum $l$. Na prática, a medida $\mu$ é substituída pela medida empírica

$$
\hat{\mu}_{n}\left(I_{j, k}\right)=n^{-1} \sum_{t=1}^{n} I\left(X_{t} \in I_{j, k}\right)=2^{-j}
$$

Neste caso, as equações (2.11) e (2.12) têm uma forma similar às ondaletas clássicas de Haar:

$$
\begin{gathered}
\check{\phi}_{j, k}(x)=2^{j / 2} I\left(x \in I_{j, k}\right), \\
\check{\psi}_{j, k}(x)=2^{-1 / 2}\left(\check{\phi}_{j+1,2 m}(x)-\check{\phi}_{j+1,2 m-1}(x)\right) .
\end{gathered}
$$

Equações análogas a essas podem ser obtidas substituindo $I_{j, k}$ por $I_{j, k}^{\circ}$.

Portanto, uma função $f(x)$ pode ser representada como a série infinita

$$
f(x)=\sum_{k=1}^{2^{j} 0} s_{j_{0}, k}^{\circ} \check{\phi}_{j_{0}, k}^{\circ}(x)+\sum_{j \geq j_{0}} \sum_{k=1}^{2^{j}} d_{j, k}^{\circ} \check{\psi}_{j, k}^{\circ}(x),
$$

para algum nível de resolução inicial $j_{0}$, onde

$$
d_{j, k}^{\circ}=\left\langle f(x), \check{\psi}_{j, k}^{\circ}(x)\right\rangle_{d G}=\int f(x) \check{\psi}_{j, k}^{\circ}(x) d G(x),
$$

e

$$
s_{j_{0}, k}^{\circ}=\left\langle f(x), \check{\phi}_{j_{0}, k}^{\circ}(x)\right\rangle_{d G} .
$$

Se for permissível um agrupamento dos dados e se $G$ for conhecida, $d_{j, k}^{\circ}$ pode ser estimado por

$$
\hat{d}_{j, k}^{\#}=n^{-1} \sum_{t=1}^{n} Y_{t} \check{\psi}_{j, k}^{\circ}\left(X_{t}\right)
$$

e similarmente para $s_{j_{0}, k}^{\circ}$. Considerando o espaço $L_{2}(d \hat{G})$, os correspondentes coeficientes empíricos de ondaletas são dados por

$$
\hat{d}_{j, k}=n^{-1} \sum_{t=1}^{n} Y_{t} \check{\psi}_{j, k}\left(X_{t}\right)
$$

e

$$
\hat{s}_{j_{0}, k}=n^{-1} \sum_{t=1}^{n} Y_{t} \check{\phi}_{j_{0}, k}\left(X_{t}\right) .
$$


Finalmente, dado um conjunto de dados $\left\{X_{t}, Y_{t}\right\}_{t=1}^{n}$, o estimador de $f$ em (2.10), não-linear obtido por aplicação de limiares aos coeficientes de ondaletas, usando a base de Haar não balanceada (2.13) e (2.14) e Limiar Duro, é dado por

$$
\hat{f}(x)=\sum_{k=1}^{2^{j}} \hat{s}_{j_{0}, k} \check{\phi}_{j_{0}, k}(x)+\sum_{j=j_{0}}^{J-1} \sum_{k=1}^{2^{j}} \eta_{H}\left(\hat{d}_{j, k}, \lambda_{j, k}\right) \check{\psi}_{j, k}(x),
$$

onde $\lambda_{j, k}$ é algum limiar. Também pode-se usar Limiar Suave.

Para alcançar uma taxa de convergência quase ótima, os limiares em (2.20) são aplicados apenas a $(j, k) \in \mathcal{J}_{n}$ tal que

$$
\mathcal{J}_{n}=\left\{(j, k) \mid 2^{j} \leq C n^{1-\alpha}\right\},
$$

para algum $\alpha \in(0,1)$ convenientemente escolhido.

\subsection{Esquema de Lifting}

Para reconstruir funções em $\Lambda^{\beta}(L, B, 0)$, para $\beta \geq 1$, seria melhor usar funções escala mais suaves. Elas podem ser obtidas através de um esquema de lifting: começando com as ondaletas não balanceadas de Haar, aplicam-se passos de predição e atualização para construir pares (primal e dual, respectivamente) de bases biortogonais

$$
\left\{\phi_{j_{0}, k}, \tilde{\phi}_{j_{0}, k}\right\} \cup\left\{\psi_{j, k}, \tilde{\psi}_{j, k}\right\}, j=j_{0}, \ldots, J, j_{0} \in\{1, \ldots, J\}, k \in \mathcal{K}_{j} .
$$

Usando a base dual para analisar a função $f$ e a base primal para reconstruí-la (sintetizá-la), pode-se escrever

$$
f(x)=\sum_{k \in \mathcal{K}_{j}}\left\langle f, \tilde{\phi}_{j_{0}, k}\right\rangle_{d \hat{G}} \phi_{j_{0}, k}+\sum_{j=j_{0}}^{J} \sum_{k \in \mathcal{K}_{j}}\left\langle f, \tilde{\psi}_{j, k}\right\rangle_{d \hat{G}} \psi_{j, k}
$$

Para construir tal base, primeiramente seja $\phi_{j, k}=\tilde{\phi}_{j, k}=\check{\phi}_{j, k}$ e $\psi_{j, k}=\tilde{\psi}_{j, k}=\check{\psi}_{j, k}$. Como as ondaletas não balanceadas de Haar são ortogonais, essas bases também são biortogonais. Seja $\boldsymbol{\Phi}_{j}$ um vetor coluna contendo as funções $\left\{\phi_{j, k}, k \in \mathcal{K}_{j}\right\}$ e similarmente para $\tilde{\boldsymbol{\Phi}}_{j}, \boldsymbol{\Psi}_{j}, \tilde{\mathbf{\Psi}}_{j}$; então, por definição das ondaletas não balanceadas de Haar, existe um conjunto de filtros biortogonais $\left\{H_{j}=\check{H}_{j}, \tilde{H}_{j}=\check{H}_{j}, G_{j}=\check{G}_{j}, \tilde{G}_{j}=\check{G}_{j}\right\}$ tais que:

$$
\begin{array}{ll}
\boldsymbol{\Phi}_{j}=\boldsymbol{\Phi}_{j+1} H_{j}, & \boldsymbol{\Psi}_{j}=\boldsymbol{\Phi}_{j+1} G_{j}, \\
\tilde{\mathbf{\Phi}}_{j}=\tilde{\boldsymbol{\Phi}}_{j+1} \tilde{H}_{j}, \quad \tilde{\mathbf{\Psi}}_{j}=\tilde{\boldsymbol{\Phi}}_{j+1} \tilde{G}_{j} .
\end{array}
$$

Generalizando a transformada discreta de ondaletas da Seção 2.1, o par de filtros de análise 


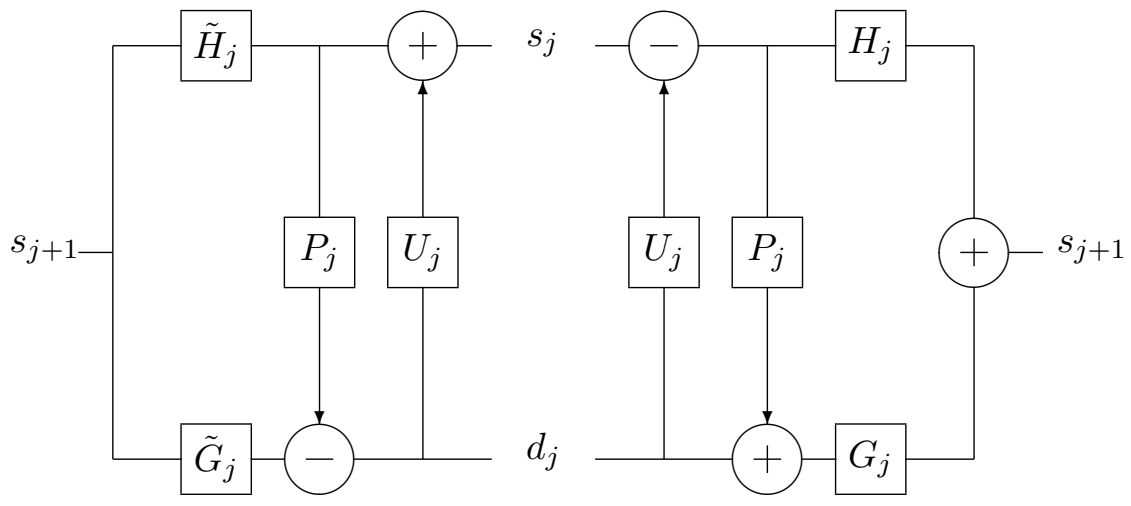

Figura 2.1: Transformadas de ondaletas de análise (esquerda) e síntese (direita) em termos de passos de lifting: $\left(\tilde{H}_{j}, \tilde{G}_{j}\right)$ são alguns filtros iniciais muito simples. No nosso caso, eles são filtros da transformada de ondaletas de Haar não balanceada. A estes filtros seguem-se passos de previsão $P_{j}$ e atualização $U_{j}$. O primeiro aumenta a suavidade de $\phi_{j_{0}, k}$, onde o último pode prover um maior número de momentos nulos, ou aumentar a estabilidade da transformada de ondaletas.

$\left(\tilde{H}_{j}, \tilde{G}_{j}\right)$ decompõem os coeficientes como

$$
s_{j}=\tilde{H}_{j}^{*} s_{j+1} \text { e } d_{j}=\tilde{G}_{j}^{*} s_{j+1},
$$

onde $s_{j}=\left\{s_{j, k}, k \in \mathcal{K}_{j}\right\}$ e similarmente para $d_{j}$. Por outro lado, o par de filtros $\left(H_{j}, G_{j}\right)$ é usado para reconstrução (ou síntese):

$$
s_{j+1}=H_{j} s_{j}+G_{j} d_{j}
$$

O lifting dual é usualmente designado a aumentar a suavidade de $\phi_{j_{0}, k}$, dado um operador de previsão $P_{j}$. Já o lifting primal é usualmente designado a aumentar a estabilidade da transformada, dado um operador $U_{j}$.

Iniciando com um par de filtros biortogonais $\left(H_{j}^{\circ}, \tilde{H}_{j}^{\circ}, G_{j}^{\circ}, \tilde{G}_{j}^{\circ}\right)$, os liftings dual e primal sempre produzem novos filtros biortogonais $\left(H_{j}, \tilde{H}_{j}, G_{j}, \tilde{G}_{j}\right)$ que são relacionados a novas bases biortogonais (SWELDEns, 1997, Teorema 8.1). Também há algoritmos para transformadas rápidas de análise e de síntese (DELOUILLE, 2002, Algoritmos 2.2.2 e 2.2.3), como ilustrado na Figura 2.1.

\subsubsection{Passo de Previsão}

Na prática, para se obter o operador $P_{j}$ primeiro obtém-se a nova matriz $H_{j}$ através do esquema de interpolação média e, então, usa-se o fato de que, pela biortogonalidade dos filtros iniciais, $P_{j}=\breve{G}_{j}^{*} H_{j}$ (DELOUILLE, 2002, eq. (4.2)).

Em um esquema de interpolação média de ordem $p$, cada coeficiente de nível mais fino $a_{j+1, l}$ é 
calculado de um conjunto de $p=2 d+1$ consecutivos coeficientes de nível mais grosso. Os intervalos associados são escolhidos tão simetricamente quanto possível em torno de $I_{j+1, l}$. O procedimento completo é o seguinte.

Para cada nível $j=J-1, \ldots, j_{0}$, faça primeiro uma transformada não balanceada de Haar, gerando os vetores $\check{s}_{j}$ e $\check{d}_{j}$, onde $\check{s}_{j}=\left\{\check{s}_{j, k}\right\}_{k \in \mathcal{K}_{j}}, \check{d}_{j}=\left\{\check{d}_{j, k}\right\}_{k \in \mathcal{K}_{j}}$. Então, para cada $l \in \mathcal{K}_{j+1}$, seja $I_{j, k(l)}$ o intervalo mais próximo de $I_{j+1, l}$ para o qual existam intervalos $I_{j, k(l)-d}$ e $I_{j, k(l)+d}$. Encontre os coeficientes $\left(c_{0}, \ldots, c_{N}\right)$ do sistema de equações

$$
\check{s}_{j, k(l)+i}=\frac{1}{n} \sum_{X_{t} \in I_{j, k(l)+i}} \frac{c_{0}+c_{1} X_{t}+\cdots+c_{N} X_{t}^{N-1}}{\hat{\mu}_{n}\left(I_{j, k}(l)+i\right)^{1 / 2}}, \quad-d \leq i \leq d,
$$

e os utilize para calcular o valor interpolado médio do nível mais fino seguinte:

$$
\begin{aligned}
a_{j+1, l} & =\frac{1}{n} \sum_{X_{t} \in I_{j+1, l}} \frac{c_{0}+c_{1} X_{t}+\cdots+c_{N} X_{t}^{N-1}}{\hat{\mu}_{n}\left(I_{j+1, l}\right)^{1 / 2}} \\
& =\left\langle c_{0}+c_{1} x+\cdots+c_{N} x^{N-1}, \check{\phi}_{j+1, l}\right\rangle_{d \hat{G}} .
\end{aligned}
$$

Pode-se mostrar que (DELOUILLE, 2002, Seção 4.1.1.2)

$$
P_{j} \check{s}_{j}=P_{j} \check{H}_{j}^{*} \check{s}_{j+1}=\check{G}_{j}^{*} H_{j} \check{H}_{j}^{*} \check{s}_{j+1}=\check{G}_{j}^{*} a_{j+1},
$$

onde $a_{j+1}=\left\{a_{j+1, l}\right\}_{l \in \mathcal{K}_{j+1}}$.

Portanto, aplicando $P_{j}$ a $\check{s}_{j}$, como no esquema de lifting da Figura 2.1, obtém-se a sequência $p_{j}$, cujos componentes $p_{j, k}$ são a diferença entre dois coeficientes sucessivos $a_{j+1, l+1}$ e $a_{j+1, l}$, onde $I_{j, k}=$ $I_{j+1, l} \cup I_{j+1, l+1}$. Os novos coeficientes de detalhes $d_{j, k}$ são então computados como $d_{j, k}=\check{d}_{j, k}-p_{j, k}$.

\subsubsection{Passo de Atualização}

Após o passo de previsão por interpolação média, a base não balanceada de Haar não é mais ortogonal. Como a igualdade de Parseval não é necessariamente verdadeira para bases biortogonais, se a base estiver muito distante da ortogonalidade, uma pequena perturbação no valor de um coeficiente de ondaleta pode causar uma importante mudança na função reconstruída. Portanto, o passo de atualização é usualmente utilizado para melhorar a estabilidade da transformada.

Esse passo é sempre recomendado mas será desconsiderado porque após o passo de previsão, e antes do passo de atualização, as ondaletas duais $\tilde{\psi}_{j, k}$ são constantes por partes (DELOUILLE, 2002, eq. (4.20)) e, se aplicarmos um passo estabilizante de atualização, em geral não há mais uma expressão analítica para as ondaletas duais.

Simulações têm mostrado que o passo estabilizante de atualização melhora a qualidade dos estimadores (SIMOENS; VANDEWALLE, ) mas provas rigorosas de suas boas propriedades ainda não existem. 
Nos resultados desta tese, será considerado que apenas o passo de previsão é implementado.

\subsection{Prova do Resultado (2.6)}

Geralmente, como prova deste resultado, é citado (LEADBETTER; LINDGREN; ROOTZÉN, 1986), mas suas demonstrações em geral são complexas. Um argumento indireto, porém mais simples é apresentado por Johnstone e Silverman (1997). Aqui apresenta-se um demonstração que pretende ser direta e simples.

Deseja-se provar que

$$
P\left(\max _{1 \leq i \leq n}\left|X_{i}\right| \geq \sqrt{2 \log n}\right) \rightarrow 0, \quad n \rightarrow \infty
$$

onde $X_{i} \sim N(0,1)$, para todo $i=1, \ldots, n$, e estas variáveis possam ser independentes ou não:

$$
\begin{aligned}
P\left(\max _{1 \leq i \leq n}\left|X_{i}\right| \geq a\right) & =1-P\left(\left|X_{1}\right|<a, \ldots,\left|X_{n}\right|<a\right) \\
& =P\left(\left[\cap_{i=1}^{n}\left\{\left|X_{i}\right|<a\right\}\right]^{c}\right) \\
& =P\left(\cup_{i=1}^{n}\left\{\left|X_{i}\right|<a\right\}^{c}\right) \\
& \leq \sum_{i=1}^{n} P\left(\left|X_{i}\right| \geq a\right) \\
& =2 \sum_{i=1}^{n} P\left(X_{i} \geq a\right), \quad \forall a>0 \\
& =2 n P\left(X_{i} \geq a\right) \\
& =2 n \int_{a}^{\infty} \frac{e^{-u^{2} / 2}}{\sqrt{2 \pi}} d u \\
& \leq 2 n \int_{a}^{\infty} \frac{u}{a} \frac{e^{-u^{2} / 2}}{\sqrt{2 \pi}} d u \\
& =2 n \int_{a^{2} / 2}^{\infty} \frac{1}{a} \frac{e^{-v}}{\sqrt{2 \pi}} d v, \quad v=u^{2} / 2 \\
& =\left.2 n \frac{-e^{-v}}{a \sqrt{2 \pi}}\right|_{a^{2} / 2} ^{\infty} \\
& =2 n \frac{e^{-a^{2} / 2}}{a \sqrt{2 \pi}} .
\end{aligned}
$$

Agora, fazendo $a=\sqrt{2 \log n}$, então

$$
\begin{aligned}
P\left(\max _{1 \leq i \leq n}\left|X_{i}\right| \geq a\right) & \leq 2 n \frac{e^{-(\sqrt{2 \log n})^{2} / 2}}{\sqrt{2 \log n} \sqrt{2 \pi}} \\
& =\frac{1}{\sqrt{\pi \log n}} \rightarrow 0, \quad n \rightarrow \infty .
\end{aligned}
$$




\section{Capítulo 3}

\section{Delineamento Fixo}

Este capítulo generaliza a metodologia de Cai e Brown (1998), para estimação via ondaletas em amostras desigualmente espaçadas, mas na presença de erros estacionários Gaussianos correlacionados. Incluem-se os casos de sinais amostrados de modo desigualmente espaçado e de regressões com delineamento fixo. Se a função verdadeira for membro de uma classe de Hölder por partes, mostra-se que o procedimento é quase ótimo. Mesmo para erros com memória longa, a taxa de convergência do procedimento é quase minimax relativamente ao caso de erros i.i.d. Como o procedimento VisuShrink é um caso especial dessa metodologia, esses resultados permanecem válidos para ele. Simulações e uma aplicação ilustram a metodologia.

\subsection{Modelo e Procedimento}

Considere o modelo

$$
Y_{i}=f\left(x_{i}\right)+\epsilon_{i}
$$

onde $f \in \Lambda^{\alpha}(M, B, m),\left\{\epsilon_{i}\right\}_{i=1}^{n}$ é oriundo de um processo estacionário gaussiano de média zero, os $x_{i}$ são valores fixos do intervalo $[0,1]$, para $i=1, \ldots, n$ e os valores $x_{i}$ ordenados $x_{(1)}<x_{(2)}<\cdots<x_{(n)}$, $n=2^{J}, J \in \mathbb{Z}$, são tais que $x_{(i)}=H^{-1}(i / n)$ para alguma função distribuição $H$ estritamente crescente em $[0,1]$. Ordene os valores de $Y_{i}$, dos pares $\left\{\left(Y_{1}, x_{1}\right), \ldots,\left(Y_{n}, x_{n}\right)\right\}$, de acordo com os $x_{i}$. Suponha que $H^{-1} \in \Lambda^{1}(h)$, onde $\Lambda^{1}(h)$ denota a classe de funções Lipschitz $f$ satisfazendo $|f(x)-f(y)| \leq h|x-y|$, para $x, y \in[0,1]$ e alguma constante $h$.

Note que $f(x)=f\left(H^{-1}(H(x))\right)$ e que a amostra refere-se à função $f\left(H^{-1}(u)\right), u \in[0,1]$, observada nos pontos igualmente espaçados $\{i / n\}_{i=1}^{n}$. Seja $f\left(H^{-1}(x)\right)$ aproximada por (CAI; BROWN, 1998, Lema 2)

$$
\tilde{g}(x)=n^{-1 / 2} \sum_{i=1}^{n} Y_{(i)} \phi_{J, i-1}(x),
$$

onde $Y_{(i)}$ denota a variável aleatória $Y_{i}$ no ponto $x_{(i)}$. Então use a projeção de $\tilde{g}(H(x))$ no espaço de multirresolução $V_{J}$ como a aproximação da função $f$ com ruído. Aplique limiares duros ou suaves aos coeficientes e então obtenha a aproximação para a função $f$ sem ruído. 
A ideia é tratar os dados como igualmente espaçados apenas na escala mais grossa e obter os coeficientes exatos nos pontos do delineamento para as escalas mais finas.

Especificamente, em um caso sem ruído, seja

$$
c_{J k}^{\prime}=n^{-1 / 2} \sum_{i=1}^{2^{J}} f\left(x_{(i)}\right)\left\langle\phi_{J, i-1} \circ H, \phi_{J, k}\right\rangle,
$$

$k=0, \ldots, 2^{J}-1$, obtido de acordo com $(2.2)$ e seja

$$
\tilde{f}(x)=\sum_{k=0}^{2^{J}-1} c_{J k}^{\prime} \phi_{J, k}(x)
$$

uma aproximação da função $f$. Note que $\tilde{f} \in V_{J}$ e, uma vez que o termo de erro não foi usado até agora, então sob as condições acima, pode-se mostrar (CAI; BROWN, 1998, Teorema 1) que

$$
\sup _{f \in \Lambda^{\alpha}(M, B, m)}\left\|f_{n}-f\right\|_{2}^{2}=o\left(n^{-2 \alpha /(1+2 \alpha)}\right)
$$

onde a quantidade máxima de pontos de descontinuidade é igual a $m=C n^{\gamma}$ com constantes $C>0$ e $0<\gamma<1 /(1+2 \alpha)$.

No caso com ruído, seja

$$
\tilde{f}_{J}(x)=\operatorname{Proj}_{V_{J}} \tilde{g}(H(x))=\sum_{k=0}^{2^{j_{0}-1}} \tilde{c}_{j_{0}, k} \phi_{j_{0}, k}(x)+\sum_{j=j_{0}}^{J-1} \sum_{k=0}^{2^{j}-1} \tilde{d}_{j, k} \psi_{j, k}(x)
$$

onde, através de (2.2), similarmente a (3.2) mas no caso com ruído,

$$
\begin{gathered}
\tilde{c}_{j_{0}, k}=n^{-1 / 2} \sum_{i=1}^{n} Y_{(i)}\left\langle\phi_{J, i-1} \circ H, \phi_{j, k}\right\rangle, \\
\tilde{d}_{j, k}=n^{-1 / 2} \sum_{i=1}^{n} Y_{(i)}\left\langle\phi_{J, i-1} \circ H, \psi_{j, k}\right\rangle, \quad k=0, \ldots, 2^{j}-1 .
\end{gathered}
$$

Aplicando-se limiares suaves $\lambda_{j, k}$ aos coeficientes de detalhe $\tilde{d}_{j, k}$, tem-se que $\hat{c}_{j_{0}, k}=\tilde{c}_{j_{0}, k}$ e $\hat{d}_{j, k}=$ $\eta_{S}\left(\tilde{d}_{j, k}, \lambda_{j, k}\right)$, Então um estimador de ondaletas de $f$, obtido por limiar suave é dado por

$$
\hat{f}(x)=\sum_{k=0}^{2^{j_{0}-1}} \hat{c}_{j_{0}, k} \phi_{j_{0}, k}(x)+\sum_{j=j_{0}}^{J-1} \sum_{k=0}^{2^{j-1}} \hat{d}_{j, k} \psi_{j, k}(x) .
$$

O limiar $\lambda_{j, k}$ será discutido nas Seções 3.2 e 3.3 . 


\subsection{Suposições e Resultados Gerais}

As seguintes suposições serão usadas no restante deste capítulo:

S1 os dados observados provêm do modelo válido (3.1);

S2 $\epsilon_{i} \sim N\left(0, \sigma_{\epsilon}^{2}\right)$ e $\operatorname{Cov}\left(\epsilon_{i}, \epsilon_{j}\right) \leq \sigma_{\epsilon}^{2}$ para todo $i, j=1,2, \ldots, n$; processos fracamente estacionários satisfazem esta condição;

S3 existe uma função distribuição $H:[0,1] \mapsto[0,1]$ estritamente crescente e conhecida; sua inversa $H^{-1} \in \Lambda^{1}(h)$ é tal que $x_{(i)}=H^{-1}(i / n), i=1,2, \ldots, n$ e $0<x_{(1)}<x_{(2)}<\cdots<x_{(n)}=1 ;$

S4 as ondaletas periodizadas $\phi$ e $\psi$ são tais que $\int \phi(t) d t=1, \psi$ é $r$-regular e de suporte compacto em $[0, N]$ e elas geram uma base ortonormal de $L_{2}[0,1]$.

Note que a suposição S3 implica que o domínio $\operatorname{Dom}\left(H^{-1}\right) \supset(0,1)$. Pelo teorema de Stepanov (HEINONEN, 2004) e suposição (S3) novamente, $H^{-1}$ tem derivadas $0<\tilde{h}(x) \leq h$ em quase todo $x \in(0,1)$, onde $\tilde{h}(x)=d H^{-1}(x) / d x$.

O primeiro resultado que será discutido é relacionado ao limiar $\lambda_{j, k}$ da Seção 3.1. As provas são dadas na Seção 3.4.

Proposição 1 Sob as suposições $\mathbf{S} 1$ a $\mathbf{S} 4$, o coeficiente empírico de ondaletas proposto em (3.4) é tal que

$$
\sigma_{j, k}^{2}=\operatorname{Var}\left(\tilde{d}_{j, k}\right) \leq n^{-1} \sigma_{\epsilon}^{2}(2 N+1) h_{j, k}=u_{j, k}^{2},
$$

onde $h_{j, k}=\operatorname{ess.sup}\left\{\tilde{h}(x): x \in \operatorname{supp}\left\{\psi_{j, k}\right\}\right\}$.

Note que este é o limite superior para $\sigma_{j, k}^{2}$ no caso de erros i.i.d. multiplicado por uma constante (CAI; BROWN, 1998).

Usando este resultado, pode-se fixar o limiar

$$
\lambda_{j, k}=u_{j, k} \sqrt{2 \log n}
$$

para uso no estimador (3.5).

O estimador final (3.5) possui uma propriedade de suavidade no sentido de que se a verdadeira função for identicamente igual a zero $(f \equiv 0)$ então $\hat{f}$ também será a função zero com alta probabilidade; isto é, existe uma sequência de constantes $P_{n}$ tais que (CAI; BROWN, 1998)

$$
P(\hat{f} \equiv 0) \geq P_{n} \rightarrow 1 \text { as } n \rightarrow \infty,
$$

Além disso, o estimador remove ruído puro com alta probabilidade quando a função $f$ pertence a alguma das classes de Hölder por partes $\Lambda^{\alpha}(M, B, m)$, onde a quantidade máxima de pontos de 
descontinuidade pode crescer polinomialmente com o tamanho da amostra. Então, nesse sentido, a estimativa $\hat{f}$ é quase ótima. Como de $\sigma_{j, k}^{2} \leq n^{-1} \sigma_{\epsilon}^{2}(2 N+1) h_{j, k}=o\left(n^{-2 \alpha /(1+2 \alpha)}\right)$, então as provas dos teoremas 3 e 4 dados por Cai e Brown (1998) pemanecem inalteradas. Essas provas baseiam-se na ortonormalidade das bases de ondaletas e não são influencidas pelas correlações dos erros. Suas provas fornecem o seguinte resultado:

Teorema 2 Seja $\hat{f}$ o estimador de limiar suave dado por (3.5) juntamente com os limiares (3.7). Então, sob as suposiçôes $\mathbf{S 1}$ a $\mathbf{S 4}$,

1. o estimador $\hat{f}$ é quase ótimo:

$$
\sup _{f \in \Lambda^{\alpha}(M, B, m)} E\left(\|\hat{f}-f\|_{2}^{2}\right) \leq C\left(\frac{\log n}{n}\right)^{2 \alpha /(1+2 \alpha)}(1+o(1))
$$

para todo $0<\alpha<r$ e todo $m \leq C n^{\gamma}$ com constantes $C>0$ e $0<\gamma<1 /(1+2 \alpha)$;

2. para qualquer valor fixo $x_{0} \in[0,1]$

$$
\sup _{f \in \Lambda^{\alpha}(M, B, m)} E\left(\left(\hat{f}\left(x_{0}\right)-f\left(x_{0}\right)\right)^{2}\right) \leq C\left(\frac{\log n}{n}\right)^{2 \alpha /(1+2 \alpha)}(1+o(1))
$$

para todo $0<\alpha<r$.

Este teorema fornece a taxa de convergência de estimação global e de estimação em um ponto. Para estimação em um ponto argumenta-se (CAI; BROWN, 1998) que a taxa adaptativa minimax é $(\log n / n)^{2 \alpha /(1+2 \alpha)}$, para o caso i.i.d. O estimador proposto em (3.5) atinge a taxa minimax para estimação de uma função em uma classe de Hölder em um ponto fixo. Este resultado permite que a quantidade de descontinuidades cresça polinomialmente com o tamanho da amostra, como em Cai e Brown (1998).

Note que a taxa de convergência minimax usual no caso de memória longa é $n^{-2 \alpha \beta /(2 \alpha+\beta)}$, onde $0<\beta<1$ é tal que $\operatorname{Cov}\left(\epsilon_{i}, \epsilon_{j}\right)=C|i-j|^{-\beta}$ (OPSOMER; WANG; YANG, 2001). O estimador proposto atinge a taxa minimax do caso i.i.d. mesmo na presença de memória longa.

\subsection{Discussão e Implementação Prática}

Seguindo a mesma discussão em Cai e Brown (1998), toda modelagem prévia foi feita com a função $H$ estritamente crescente e $H^{-1} \in \Lambda^{1}(h)$. Como na prática $H$ é usualmente desconhecida, pode-se usar uma função empírica $\hat{H}_{n}$ linear por partes satisfazendo $\hat{H}\left(x_{(i)}\right)=i / n$. Além dessa função ser de fácil implementação, todos os resultados teóricos permanecem válidos (CAI; BROWN, 1998). Os demais aspectos relacionados à implementação computacional permanecem inalterados em relação aos descritos em Cai e Brown (1998) e não serão repetidos aqui. É suficiente notar que o procedimento completo envolve dois passos adicionais além da aplicação de limiares aos coeficientes de ondaletas no 
caso igualmente espaçado: um precondicionamento e um pós-condicionamento feito por multiplicação de matrizes. Mais importante, a dimensão das matrizes não depende do tamanho da amostra.

Em relação à estimação de $\sigma_{\epsilon}^{2}$, a sugestão é que se use uma estimação-piloto (ALTMAN, 1990) aplicando limiares aos coeficientes de ondaletas por níveis, tratando os dados como se fossem igualmente espaçados. Então obtenha a estimativa $\hat{\sigma}_{\epsilon}^{2}$ como a variância dos resíduos dessa estimação-piloto.

O procedimento VisuShrink (DONOHO; JOHnStone, 1994, 1995) é um caso especial do método proposto quando as observações são igualmente espaçadas, uma vez que $H$ é a identidade e $h=1$. Portanto, os resultados da Seção 3.2 devem continuar válidos. Quando os erros são correlacionados, a variância dos coeficientes empíricos é diferente ao longo dos níveis (JOHNSTONE; SILVERMAN, 1997), e esse fato parece ser capturado por $h_{j, k}$ em (3.6).

De modo a se visualizar como as taxas de convergência no caso de erros correlacionados se relacionam com a do caso i.i.d., uma pequena simulação foi realizada. A função $f\left(x_{i}\right)=\operatorname{sen}\left(2 \pi x_{i}\right)$, foi amostrada em pontos igualmente espaçados $x_{i} \in[0,1], i=1,2, \ldots, n$ para tamanhos de amostras de $n=2^{4}=16$ até $n=2^{20}=1048576$. Um ruído gaussiano de memória longa com coeficiente de Hurst $H=0,9$ foi adicionado a esta função. Uma estimativa $\hat{f}_{\text {corr }}\left(x_{i}\right), i=1,2, \ldots, n$ foi obtida por aplicação do procedimento VisuShrink por níveis, nesses dados simulados. Isso foi replicado 300 vezes e, em cada vez, a quantidade

$$
E C_{r}=\sum_{i=1}^{n}\left(\hat{f}_{\text {corr }}\left(x_{i}\right)-f\left(x_{i}\right)\right)^{2}, \quad r=1,2, \ldots, 300,
$$

foi calculada, como aproximação do respectivo IMSE. O mesmo foi feito com ruído i.i.d. $N\left(0, \sigma^{2}\right)$, onde $\sigma^{2}$ é a variância do ruído de memória longa. As respectivas estimativas $\hat{f}_{\text {ind }}\left(x_{i}\right)$ geraram as quantidades

$$
E I_{r}=\sum_{i=1}^{n}\left(\hat{f}_{\text {ind }}\left(x_{i}\right)-f\left(x_{i}\right)\right)^{2}, \quad r=1,2, \ldots, 300 .
$$

Se os valores esperados dessas duas quantidades diferirem por uma constante multiplicativa, então a razão $C(n)=\left(\sum_{r} E C_{r}\right) /\left(\sum_{r} E I_{r}\right)$ deveria estimar sua magnitude relativa, para $n$ grande. Portanto, pelo resultado do Teorema 2, espera-se que um gráfico de $C(n) \times n$ seja limitado superiormente. Gráficos desse tipo para $H=0,6$ e $H=0,9$ são mostrados na Figura 3.1. As simulações foram feitas usando a linguagem R (R Development Core Team, 2005) através dos pacotes fSeries (WUERTZ, 2006) e Wavethresh (NASON; KOVAC; MAECHLER, 2006).

Na Figura 3.1 as constantes do caso de memória longa são realmente maiores do que no caso i.i.d., mas aparentemente limitadas superiormente. Isso é uma indicação empírica de que as taxas de convergência são as mesmas em ambos os casos. Note que as constantes são maiores quando a memória é mais longa $(H=0,9)$ mas, quando $n=2^{J}$ aumenta, as constantes parecem convergir para 1. 

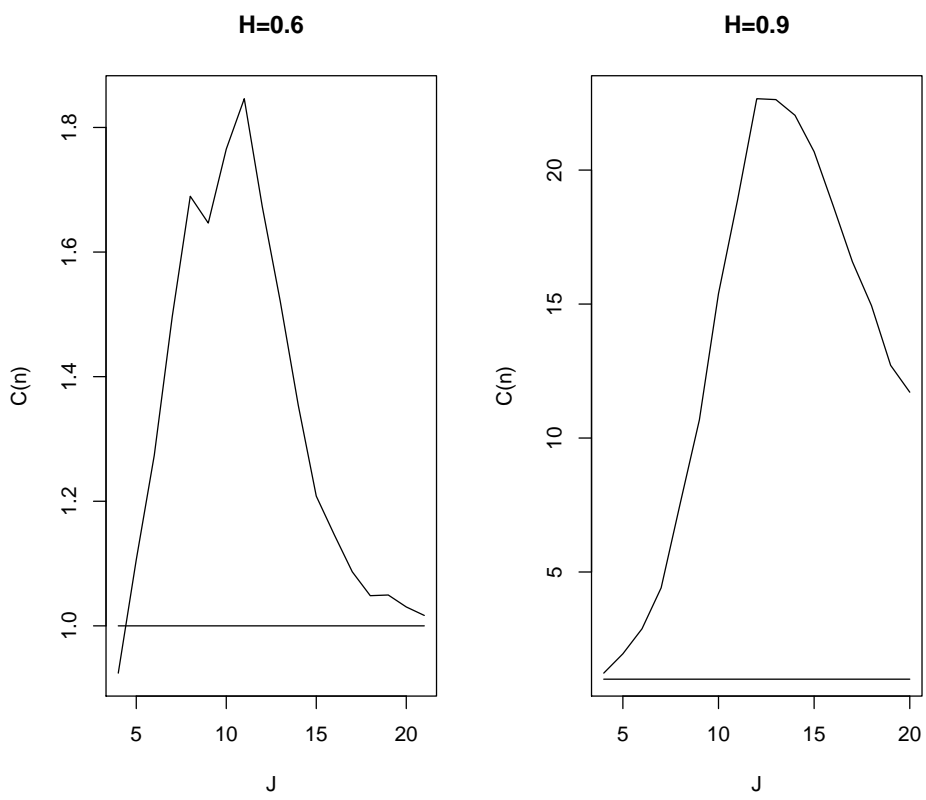

Figura 3.1: Razão estimada $C(n)$ entre os riscos quadráticos de norma $L_{2}$ dos dados de memória longa (coeficientes de Hurst $H=0,6$ e $H=0,9)$ e dos dados i.i.d.

\subsubsection{Simulações com Amostras Finitas}

Algumas simulações foram feitas para ilustrar a suavidade alcançada pelo método proposto contra um competidor que é tratar amostras desigualmente espaçadas como se elas fossem igualmente espaçadas. Este competidor assume que a amostra é igualmente espaçada mas não atinge taxas de convergência ótimas em alguns casos (CAI, 1996) i.i.d. Novamente, todas as simulações foram feitas usando a linguagem R (R Development Core Team, 2005) através do pacote Wavethresh (NASON; KOVAC; MAECHLER, 2006).

Em todos os casos, os dados $\left(y_{1}, x_{1}\right),\left(y_{2}, x_{2}\right), \ldots,\left(y_{n}, x_{n}\right)$ foram gerados a partir de um modelo da forma (3.1) onde $\epsilon_{i}$ seguiu o processo $\operatorname{ARMA}(2,2)$

$$
\epsilon_{i}=0,8897 \epsilon_{i-1}-0,4858 \epsilon_{i-2}+a_{i}-0,2279 a_{i-1}+0,2488 a_{i-2},
$$

onde $a_{i} \sim N\left(0, \sigma^{2}\right)$ com diferentes valores de $\sigma^{2}$ dependendo do caso. Também, $0<x_{1}<x_{2}<\cdots<$ $x_{n}=1$ e $n=256$. A base de ondaletas Symmlet 16 foi usada em todos os casos.

A amostra desigualmente espaçada foi obtida do seguinte modo. Primeiro foram gerados 512 pontos $x$ onde a função seria amostrada, seguindo uma distribuição Uniforme em $[0,1]$. Então, foi mantida uma proporção $p=0,3$ predefinida de pontos em cada um dos intervalos $(0,1,0,2],(0,5,0,6]$ e $(0,8,0,9]$. Depois disso, os primeiros 256 pontos gerados foram mantidos e os restantes descartados. Finalmente, os valores $x$ foram ordenados e os valores $y$ foram calculados sem ruído. Estes pontos 
de delineamento são gerados uma única vez, tornando-os fixos nas simulações. Usando este único delineamento, nas simulações, ruídos gerados seguindo (3.11) são adicionados aos valores $y$.

As seguintes funções foram usadas para simulação. Algumas motivações para cada uma delas são dadas juntamente com os valores $\sigma$ e o primeiro nível de resolução ao qual foram aplicados limiares suaves.

1. Seno: esta é uma função seno usual $f(x)=\operatorname{sen}(2 \pi x)$ usada em Cai e Brown (1998) e pode ser considerada como referência $\left(\sigma=0,1,3^{\circ}\right.$ nível, Figura 3.2).

2. HeaviSine: esta função $f(x)=\operatorname{sen}(2 \pi x)-0,5 \operatorname{sgn}(x-0,3)-0,5 \operatorname{sgn}(0,72-x)$ é a função seno com dois saltos, usada para se avaliar o efeito das descontinuidades em uma função usual $(\sigma=0,1$, $6^{\circ}$ nível, Figura 3.3).

3. Seno transladada no tempo: $f(x)=0,3 \operatorname{sen}(3 \pi[g(g(g(g(x))))+x])+0,5$, onde $g(x)=(1-$ $\cos (\pi x)) / 2$. É diferente de uma combinação linear de senoides e pode ser vista como representando o tipo de curva que seria usualmente considerada para estimação $\left(\sigma=0,05,5^{\circ}\right.$ nível, Figura 3.4).

Na Figura 3.2, tratar dados desigualmente espaçados da função seno como se não o fossem, leva a uma estimativa mais enrugada que a obtida pelo método proposto.

Para a função HeaviSine, estimadores igualmente suaves são obtidos em ambos os casos (Figura 3.3), mas aparentemente o método proposto é pior para detectar a descontinuidade do lado direito da função.

Na Figura 3.4, é evidente a suavidade e o melhor ajuste obtido pelo método proposto.

Como uma regra geral, o método proposto mostra um comportamento melhor do que simplesmente assumindo que os dados são igualmente espaçados e aplicando limiares suaves por níveis (JOHNSTONE; SILVERMAN, 1997).

\subsubsection{Aplicação}

Em Economia, há certa preocupação com perfis de renda-idade por país, gênero etc. Tradicionalmente, a especificação das relações entre renda média e idade (como uma aproximação para experiência) tem sido parametrizada por uma curva quadrática na idade com uma variável adicional para educação (HECKMAN; POLACHECK, 1974). Essa relação paramétrica quadrática entre ganhos e idade tem sido questionada por alguns autores que recentemente têm usado estimação não-paramétrica (PAGAN; ULLAH, 1999).

Para os propósitos desta tese, os dados usados foram de mulheres brasileiras com mestrado ou doutorado. Os dados são oriundos da Pesquisa Nacional por Amostras de Domicílios (PNAD) 2004, produzida pela Fundação Instituto Brasileiro de Geografia e Estatística (IBGE). A renda foi definida 
(a)

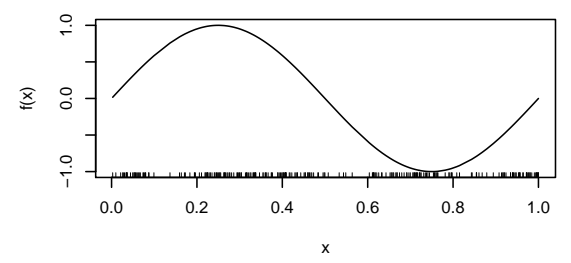

(c)

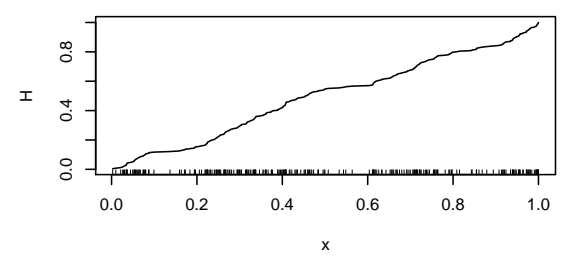

(e)

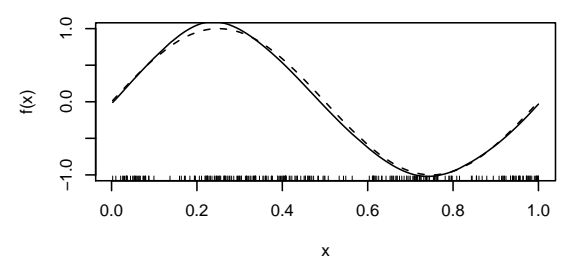

(b)

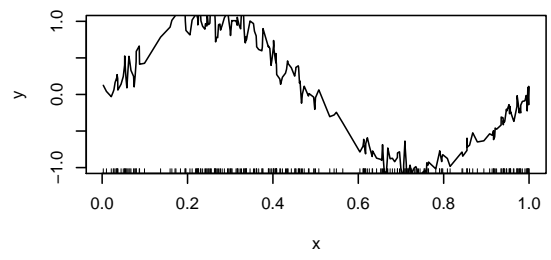

(d)

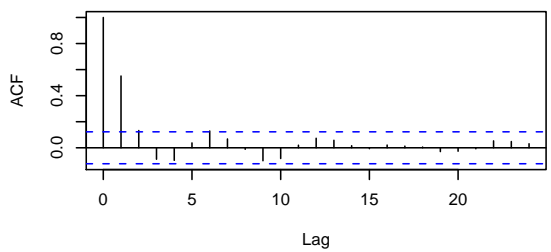

(f)

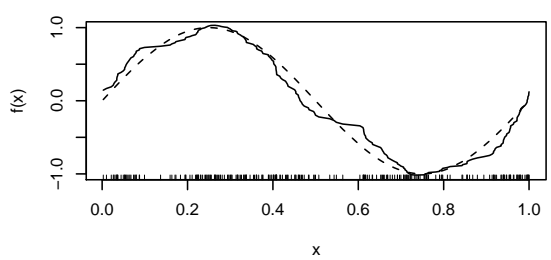

Figura 3.2: (a) função seno; (b) amostra com ruído de desvio padrão $\sigma=0,1$; (c) função distribuição $H$; (d) autocorrelograma; (e) estimador proposto (linha cheia) e função original (linha tracejada), limiar a partir do $3^{\circ}$ nível; (f) estimador tratando como se a amostra fosse igualmente espaçada (linha cheia) e função original (linha tracejada), limiar a partir do $3^{\circ}$ nível.

como o logaritmo natural dos rendimentos do trabalho principal por hora trabalhada. Idade foi definida como o número de anos desde o nascimento até a data de referência da PNAD 2004. A idade foi calculada com duas casas decimais, gerando a dados desigualmente espaçados. Como apenas mulheres com mestrado ou doutorado foram consideradas, então a escolaridade foi considerada constante. As rendas foram padronizadas para terem média zero.

Para os 421 pontos resultantes, uma curva quadrática na idade foi ajustada por mínimos quadrados. Os resíduos mostraram estatísticas de Ljung-Box até o lag 6 e até o lag 12 significativas a 5\%. Interessantemente, esse padrão não foi observado nos dados dos homens e, por essa razão, pode-se suspeitar que haja outros fatores que são autocorrelacionados e que não foram considerados na análise dos dados das mulheres. Também, uma estimação piloto usando ondaletas com aplicação de limiar suave por níveis produziu coeficientes de ondaletas com diferentes variâncias estimadas em cada nível, como indicativo de erros correlacionados (JOHNSTONE; SILVERMAN, 1997).

Como os 421 pontos não eram potência de dois, as 256 idades centrais foram analisadas resultando em ganhos para idades entre 34,40 a 53,60 anos, aproximadamente. Uma estimativa piloto de ondaletas aplicando limiares suaves por níveis e tratando os dados como se fossem igualmente espaçados, foi 
(a)

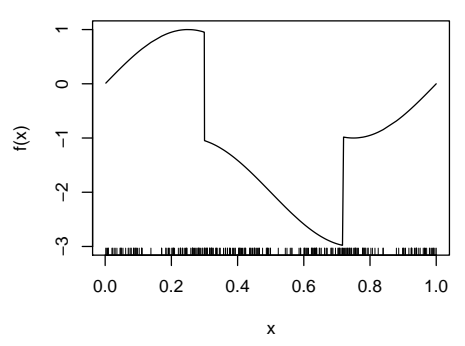

(c)

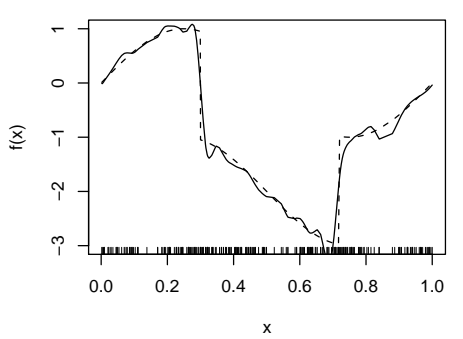

(b)

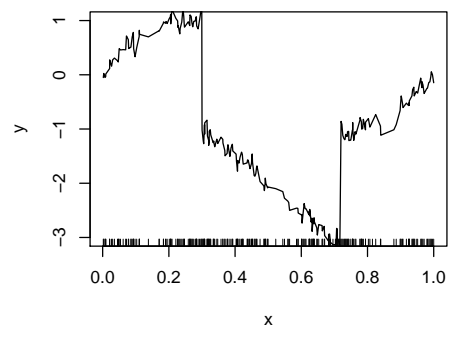

(d)

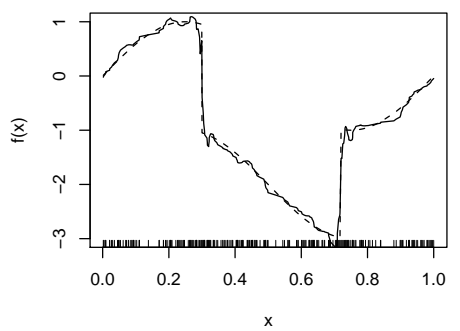

Figura 3.3: (a) função HeaviSine; (b) amostra com ruído de desvio padrão $\sigma=0,1$; (c) estimador proposto (linha cheia) e função original (linha tracejada), limiar a partir do $6^{\circ}$ nível; (d) estimador tratando como se a amostra fosse igualmente espaçada (linha cheia) e função original (linha tracejada), limiar a partir do $6^{\circ}$ nível.

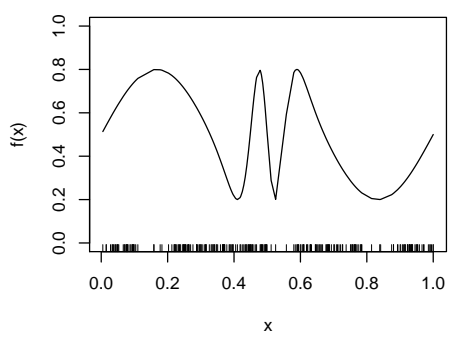

(c)

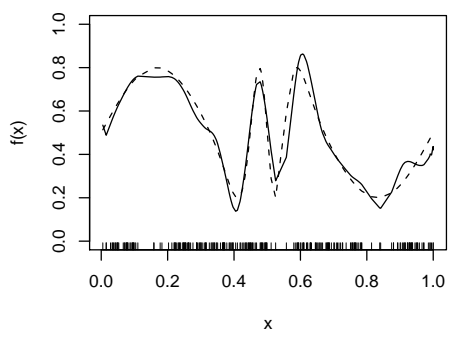

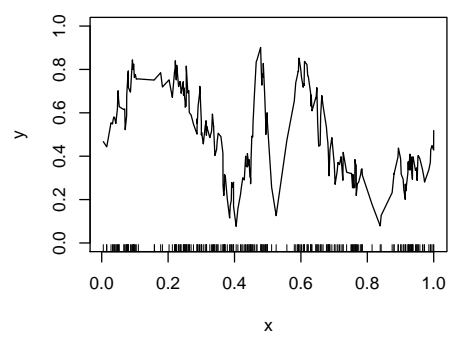

(d)

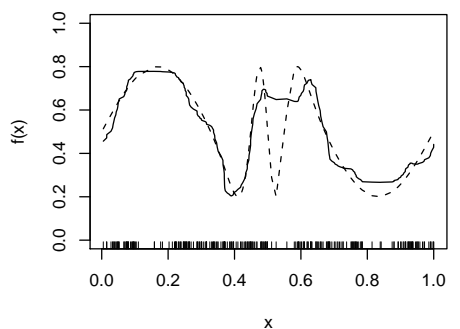

Figura 3.4: (a) função seno transladada no tempo; (b) amostra com ruído de desvio padrão $\sigma=0,05$; (c) estimador proposto (linha cheia) e função original (linha tracejada), limiar a partir do $5^{\circ}$ nível; (d) estimador tratando como se a amostra fosse igualmente espaçada (linha cheia) e função original (linha tracejada), limiar a partir do $5^{\circ}$ nível. 
feita para se estimar a variância dos erros. Essa estimativa obtida foi aplicada à metodologia descrita neste capítulo usando ondaletas Symmlet 8 e limiares suaves por níveis, a partir do nível $j_{0}=2$. A função estimada resultante e os respectivos dados são mostrados na Figura 3.5.

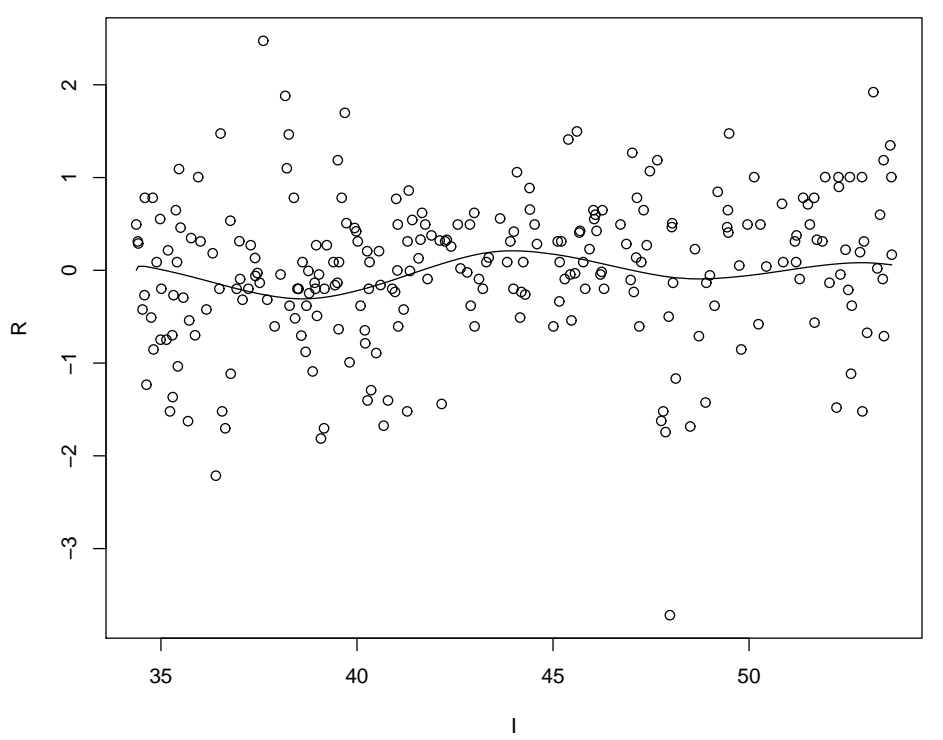

Figura 3.5: Relação entre renda $R$ e idade $I$ para mulheres brasileiras com mestrado ou doutorado, IBGE, PNAD 2004. A renda é medida pela diferença, em relação à média, do logaritmo natural dos rendimentos do trabalho principal por hora trabalhada. A idade é medida em anos completos até a data da PNAD 2004, com duas casas decimais.

Na Figura 3.5 pode-se ver a influência de um possível ponto atípico próximo à idade de 48 anos que deve ser melhor investigado. Também, há uma queda na renda por volta dos 36 e 40 anos de idade, talvez devido ao efeito da geração, pois representariam ganhos de pessoas que pertencem a gerações diferentes. Assim, esse gráfico apresentaria uma sobreposição das trajetórias de ganhos de diferentes gerações (HECKMAN; POLACHECK, 1974; PAGAN; Ullah, 1999). Se o ambiente sóciopolítico da economia tiver permanecido estável através dessas gerações, então essas trajetórias podem ser consideradas a mesma. Explicações mais detalhadas podem ser realizadas usando-se, por exemplo, dados dos homens.

Essa queda na renda é obtida a partir da especificação não-paramétrica, mas não a partir de uma especificação quadrática. A queda na renda também indica a falta de concavidade global e que outra relação pode ser mais apropriada do que a especificação quadrática. 


\subsection{Provas}

\subsubsection{Prova da Proposição 1}

$$
\begin{aligned}
\sigma_{j, k}^{2}= & \operatorname{Var}\left(\tilde{d}_{j, k}\right) \\
= & \operatorname{Var}\left(n^{-1 / 2} \sum_{i=1}^{n} Y_{(i)}\left\langle\phi_{J, i-1} \circ H, \psi_{j, k}\right\rangle\right) \\
= & \operatorname{Cov}\left(n^{-1 / 2} \sum_{i=1}^{n} Y_{(i)}\left\langle\phi_{J, i-1} \circ H, \psi_{j, k}\right\rangle, n^{-1 / 2} \sum_{m=1}^{n} Y_{(m)}\left\langle\phi_{J, m-1} \circ H, \psi_{j, k}\right\rangle\right) \\
= & n^{-1} \sum_{i=1}^{n} \sum_{m=1}^{n} \operatorname{Cov}\left(Y_{(i)}, Y_{(m)}\right)\left\langle\phi_{J, i-1} \circ H, \psi_{j, k}\right\rangle\left\langle\phi_{J, m-1} \circ H, \psi_{j, k}\right\rangle \\
= & n^{-1} \sum_{i=1}^{n} \sum_{m=1}^{n} \operatorname{Cov}\left(f\left(x_{(i)}\right)+\epsilon_{(i)}, f\left(x_{(m)}\right)+\epsilon_{(m)}\right)\left\langle\phi_{J, i-1} \circ H, \psi_{j, k}\right\rangle \times \\
& \times\left\langle\phi_{J, m-1} \circ H, \psi_{j, k}\right\rangle \quad(\text { por } \mathbf{S 1}) \\
= & n^{-1} \sum_{i=1}^{n} \sum_{m=1}^{n} \operatorname{Cov}\left(\epsilon_{(i)}, \epsilon_{(m)}\right)\left\langle\phi_{J, i-1} \circ H, \psi_{j, k}\right\rangle\left\langle\phi_{J, m-1} \circ H, \psi_{j, k}\right\rangle .
\end{aligned}
$$

Denotando $\operatorname{Cov}\left(\epsilon_{(i)}, \epsilon_{(m)}\right)$ por $\sigma_{i, m}$ e expandindo os produtos internos, a última equação é igual a

$$
n^{-1} \sum_{i=1}^{n} \sum_{m=1}^{n} \sigma_{i, m} \int \phi_{J, i-1}(H(t)) \psi_{j, k}(t) d t \int \phi_{J, m-1}(H(u)) \psi_{j, k}(u) d u
$$

Fazendo $H(u)=v$ e $d u=\tilde{h}(v) d v$, segue que

$$
\begin{aligned}
\sigma_{j, k}^{2} & =n^{-1} \sum_{i=1}^{n} \sum_{m=1}^{n} \sigma_{i, m} \int \phi_{J, i-1}(H(t)) \psi_{j, k}(t) d t \int \phi_{J, m-1}(v) \psi_{j, k}\left(H^{-1}(v)\right) \tilde{h}(v) d v \\
& =n^{-1} \int \psi_{j, k}(t) \int \psi_{j, k}\left(H^{-1}(v)\right) \tilde{h}(v) \sum_{i=1}^{n} \sum_{m=1}^{n} \sigma_{i, m} \phi_{J, i-1}(H(t)) \phi_{J, m-1}(v) d v d t
\end{aligned}
$$

Como por S4, $\operatorname{supp}\left\{\phi_{J, i-1}\right\} \cap \operatorname{supp}\left\{\phi_{J, m-1}\right\} \neq \emptyset \Leftrightarrow|i-m| \leq N$, então a última equação é igual a

$$
n^{-1} \int \psi_{j, k}(t) \int \psi_{j, k}\left(H^{-1}(v)\right) \tilde{h}(v) \sum_{i=1}^{n} \sum_{\substack{m=1 \\|i-m| \leq N}}^{n} \sigma_{i, m} \phi_{J, i-1}(H(t)) \phi_{J, m-1}(v) d v d t
$$


e fazendo $m-i=u$, segue que

$$
\begin{aligned}
\sigma_{j, k}^{2}= & n^{-1} \int \psi_{j, k}(t) \int \psi_{j, k}\left(H^{-1}(v)\right) \tilde{h}(v) \times \\
& \times \sum_{i=1}^{n} \sum_{u=-N}^{N} \sigma_{i, i+u} \phi_{J, i}(H(t)) \phi_{J, i+u}(v) d v d t \\
= & n^{-1} \int \psi_{j, k}(t) \int \psi_{j, k}\left(H^{-1}(v)\right) \tilde{h}(v) \times \\
& \times \sum_{i=1}^{n} \sum_{u=-N}^{N} \sigma_{i, i+u} \phi_{J, i-1}(H(t)) \phi_{J, i-1}(v-u / n) d v d t \\
= & n^{-1} \int \psi_{j, k}(t) \sum_{u=-N}^{N} \sum_{i=1}^{n} \sigma_{i, i+u} \times \\
& \times \int \psi_{j, k}\left(H^{-1}(v)\right) \tilde{h}(v) \phi_{J, i-1}(H(t)) \phi_{J, i-1}(v-u / n) d v d t
\end{aligned}
$$

Agora, seja

$$
\sigma(u, t, j, k)=\arg _{\sigma} \max _{i} \sum_{i=1}^{n} \sigma_{i, i+u} \int \psi_{j, k}\left(H^{-1}(v)\right) \tilde{h}(v) \phi_{J, i-1}(H(t)) \phi_{J, i-1}(v-u / n) d v .
$$

\section{Então}

$$
\sigma_{j, k}^{2} \leq n^{-1} \int \psi_{j, k}(t) \sum_{u=-N}^{N} \sigma(u, t, j, k) \sum_{i=1}^{n} \int \psi_{j, k}\left(H^{-1}(v)\right) \tilde{h}(v) \phi_{J, i-1}(v-u / n) d v \phi_{J, i-1}(H(t)) d t
$$

e transformando $v-u / n=w$, segue que

$$
\begin{aligned}
\sigma_{j, k}^{2} \leq & n^{-1} \int \psi_{j, k}(t) \sum_{u=-N}^{N} \sigma(u, t, j, k) \times \\
& \times \sum_{i=1}^{n} \int \psi_{j, k}\left(H^{-1}(w+u / n)\right) \tilde{h}(w+u / n) \phi_{J, i-1}(w) d w \phi_{J, i-1}(H(t)) d t \\
= & n^{-1} \int \psi_{j, k}(t) \sum_{u=-N}^{N} \sigma(u, t, j, k) \times \\
& \times \sum_{i=1}^{n}\left\langle\psi_{j, k}\left(H^{-1}(\cdot+u / n)\right) \tilde{h}(\cdot+u / n), \phi_{J, i-1}(\cdot)\right\rangle \phi_{J, i-1}(H(t)) d t
\end{aligned}
$$




$$
\begin{aligned}
& =n^{-1} \int \psi_{j, k}(t) \sum_{u=-N}^{N} \sigma(u, t, j, k) \psi_{j, k}\left(H^{-1}(H(t)+u / n)\right) \tilde{h}(H(t)+u / n) d t \\
& =n^{-1} \sum_{u=-N}^{N} \int \psi_{j, k}(t) \sigma(u, t, j, k) \psi_{j, k}\left(H^{-1}(H(t)+u / n)\right) \tilde{h}(H(t)+u / n) d t .
\end{aligned}
$$

Agora, seja $\sigma(u, j, k)=\arg _{\sigma} \max _{t} \int \psi_{j, k}(t) \sigma(u, t, j, k) \psi_{j, k}\left(H^{-1}(H(t)+u / n)\right) \tilde{h}(H(t)+u / n) d t$. Então a última expressão é menor ou igual a

$$
n^{-1} \sum_{u=-N}^{N} \sigma(u, j, k) \int \psi_{j, k}(t) \psi_{j, k}\left(H^{-1}(H(t)+u / n)\right) \tilde{h}(H(t)+u / n) d t,
$$

e, fazendo $\tilde{h}(u, j, k)=\arg _{\tilde{h}} \max _{t} \int \psi_{j, k}(t) \psi_{j, k}\left(H^{-1}(H(t)+u / n)\right) \tilde{h}(H(t)+u / n) d t$, então segue que

$$
\begin{aligned}
\sigma_{j, k}^{2} \leq & n^{-1} \sum_{u=-N}^{N} \sigma(u, j, k) \tilde{h}(u, j, k) \int \psi_{j, k}(t) \psi_{j, k}\left(H^{-1}(H(t)+u / n)\right) d t \\
\leq & n^{-1} \sum_{u=-N}^{N}|\sigma(u, j, k)| \tilde{h}(u, j, k)\left|\int \psi_{j, k}(t) \psi_{j, k}\left(H^{-1}(H(t)+u / n)\right) d t\right| \\
\leq & n^{-1} \sum_{u=-N}^{N}|\sigma(u, j, k)| \tilde{h}(u, j, k)\left|\int \psi_{j, k}^{2}(t) d t\right| \times \\
& \times\left|\int \psi_{j, k}^{2}\left(H^{-1}(H(s)+u / n)\right) d s\right| \text { (por Cauchy-Schwarz) } \\
= & n^{-1} \sum_{u=-N}^{N}|\sigma(u, j, k)| \tilde{h}(u, j, k) \quad \text { (por S4: ortonormalidade). }
\end{aligned}
$$

Finalmente, como $0 \leq \tilde{h}(u, j, k) \leq h_{j, k}=\operatorname{ess} \sup \left\{\tilde{h}(x): x \in \operatorname{supp}\left\{\psi_{j, k}\right\}\right\}$ e $|\sigma(u, j, k)| \leq \sigma_{\epsilon}^{2}($ por S2), para todo $u \in[-N, N]$, então resulta que

$$
\sigma_{j, k}^{2} \leq n^{-1} \sigma_{\epsilon}^{2} h_{j, k}(2 N+1)
$$

\subsubsection{Prova do Resultado (3.8)}

$$
P(\hat{f} \equiv 0) \quad \geq \sum_{j, k} P\left(\hat{d}_{j, k}=0\right)
$$




$$
\begin{aligned}
& =\sum_{j, k} P\left(\tilde{d}_{j, k} \leq \lambda_{j, k}\right) \\
& \geq P\left(\max _{j, k}\left|\tilde{d}_{j, k} / u_{j, k}\right| \leq \sqrt{2 \log n}\right)
\end{aligned}
$$

e a última expressão converge para 1 quando $n \rightarrow \infty$ de acordo com (2.6). 


\section{Capítulo 4}

\section{Delineamentos Aleatórios Uniforme e Jittered}

Neste capítulo apresentam-se alguns resultados em regressão não-paramétrica usando métodos de ondaletas na presença de erros gaussianos estacionários autocorrelacionados, e quando a variável explicativa segue uma distribuição Uniforme ou provém de uma amostragem estocástica como a jittered. O objetivo é estimar o sinal globalmente com baixo risco. Mostra-se que nesses casos especiais, as amostras podem ser tratadas como se fossem igualmente espaçadas e com ruído correlacionado; i.e., o estimador alcança uma taxa de convergência quase ótima. Algumas simulações comparam as taxas de convergência nos casos com e sem espaçamento igual, em amostras finitas. Estimativas de volatilidade diária de uma ação pouco negociada ilustra a utilidade do método.

\subsection{Introdução}

Um problema matemático de considerável interesse é aproximar uma função contínua $f(t), t \in$ $[0,1]$, baseando-se em amostras $f\left(t_{i}\right), i=1, \ldots, n$. A amostragem é muito frequentemente feita em uma grade regular $t_{i}=i /(n+1)$. Assume-se que não se observa $f\left(t_{i}\right)$ diretamente, mas apenas na presença de um ruído gaussiano $e\left(t_{i}\right)$ correlacionado e de média zero. Então, os dados consistem de pontos $\left\{\left(t_{1}, y\left(t_{1}\right)\right), \ldots,\left(t_{n}, y\left(t_{n}\right)\right)\right\}$, onde $y\left(t_{i}\right)=f\left(t_{i}\right)+e\left(t_{i}\right)$, para $i=1, \ldots, n$, e o objetivo é extrair o sinal $f(t)$ dos dados com baixo IMSE. Métodos de encolhimento de coeficientes de ondaletas têm tido sucesso em extração de sinal e regressão não-paramétrica, mas a maioria deles é focada em amostras igualmente espaçadas com erros i.i.d.

Neste capítulo, consideram-se esquemas amostrais estocásticos onde ou os pontos amostrais $t_{i}$ são Uniformemente distribuídos em $[0,1]$ ou eles provêm de um jittering; i.e., $t_{i}=i /(n+1)+j_{i}$, onde $j_{i}$ são variáveis aleatórias i.i.d. Uniforme $[-1 /(2(n+1)), 1 /(2(n+1))]$.

Técnicas de amostragem estocástica são de interesse porque podem reduzir certos problemas de aliasing associados com amostragem em uma grade regular (DIPPÉ; WOLD, 1985). Mostra-se que nestes casos, as amostras podem ser tratadas como se fossem igualmente espaçadas e com ruído correlacionado, isto é, aplica-se o procedimento VisuShrink (DONOHO; JOHNSTONE, 1994) com limiares dependentes do nível. 


\subsection{Regressão via Ondaletas com Delineamento Aleatório e Erros Correlaciona- dos}

Considere uma amostra $\left(t_{1}, y_{1}\right),\left(t_{2}, y_{2}\right), \ldots,\left(t_{n}, y_{n}\right)$ oriunda de um esquema de amostragem estocástica. Neste caso, os $t_{i}$ ou são realizações independentes de uma variável aleatória com distribuição Uniforme $[0,1]$, ou são oriundas de jittering; i.e., $t_{i}=i /(n+1)+j_{i}$, onde $j_{i}$ são variáveis aleatórias i.i.d. Uniforme $[-1 /(2(n+1)), 1 /(2(n+1))]$. Denote as estatísticas de ordem dos $t_{i}$ por $0 \leq t_{(1)}<t_{(2)}<\cdots<t_{(n)} \leq 1$. Dadas as observações assuma o modelo

$$
y_{i}=f\left(t_{(i)}\right)+e_{i}
$$

onde os erros $e_{i}=e\left(t_{(i)}\right)$ são tais que

$$
\operatorname{Cov}\left(e\left(t_{(i)}\right), e\left(t_{(j)}\right)\right)=\gamma(|i-j|), \quad \text { and } \quad \lim _{n \rightarrow \infty} \sum_{u=-(n-1)}^{n-1}|\gamma(u)|<\infty
$$

Esse tipo de correlação ocorre em diversas aplicações (veja e.g. Cochrane e Orcutt (1949), Kutner et al. (2004), Qin e Gilbert (2001)), e casos específicos, de interesse, são dados pelas seguintes

Proposição 2 Sejam $\left\{e\left(t_{(i)}\right), i=1, \ldots, n\right\}$ oriundos de um processo estocástico gaussiano e $(t), t \in$ $(0,1)$, em tempo contínuo, de média zero, do tipo AR(1). Sejam os pontos aleatórios $t_{i}=t_{(i)}=$ $i /(n+1)+j_{i}$, onde $j_{i}$ são variáveis aleatórias i.i.d. Uniforme $[-1 /(2(n+1)), 1 /(2(n+1))]$. Seja $\operatorname{Cov}\left(e\left(t_{(i)}\right), e\left(t_{(j)}\right)\right)=\sigma^{2} e^{-(n+1) \beta\left|t_{(i)}-t_{(j)}\right|}$, para algum $\beta>0,0<\sigma^{2}<\infty$ e valores fixos $i$ e $j$. Então as condiçôes (4.2) se matêm para $i, j=1, \ldots, n$.

Proposição $3 \operatorname{Sejam}\left\{e\left(t_{(i)}\right), i=1, \ldots, n\right\}$ oriundos de um processo estocástico gaussiano $e(t), t \in$ $(0,1)$, em tempo contínuo, de média zero, do tipo AR(1). Sejam os pontos aleatórios $0<t_{(1)}<\ldots<$ $t_{(n)}<1$ tais que $t_{(i)} \sim \operatorname{Beta}(i, n-i+1)$. Seja $\operatorname{Cov}\left(e\left(t_{(i)}\right), e\left(t_{(j)}\right)\right)=\sigma^{2} e^{-(n+1) \beta\left|t_{(i)}-t_{(j)}\right|}$, para algum $\beta>0,0<\sigma^{2}<\infty$ e valores fixos $i$ e $j$. Então as condiçôes (4.2) se matêm para $i, j=1, \ldots, n$.

Note que uma condição suficiente para que esse tipo de resultado seja válido, é que

$$
\operatorname{Cov}\left(e\left(t_{(i)}\right), e\left(t_{(j)}\right)\right) \leq C \sigma^{2} e^{-\beta|i-j|}
$$

para alguma constante positiva $C<\infty$.

A covariância nas Proposições 2 e 3 não é exatamente a de um processo $\operatorname{AR}(1)$ porque está se mapeando um processo na reta para o intervalo $(0,1)$, tal que a correlação entre dois pontos fixos diminui à medida em que o tamanho da amostra aumenta. Isso é necessário pois um valor fixo para $\left|t_{(i)}-t_{(j)}\right|$ corresponde a uma distância decrescente entre as observações à medida em que se obtém mais dados. 
Seja $\hat{f}(x)$ o estimador de $f(x)$ para todo $x \in[0,1]$, onde

$$
\hat{f}(x)=\sum_{k=0}^{2^{j_{0}}-1} \hat{c}_{j_{0}, k} \phi_{j_{0}, k}(x)+\sum_{j=j_{0}}^{J^{\prime}-1} \sum_{k=0}^{2^{j}-1} \hat{d}_{j, k} \psi_{j, k}(x),
$$

onde $\hat{d}_{j, k}$ é dado por (2.4) e $J^{\prime}$ é o maior inteiro tal que $2^{J^{\prime}} \leq K \sqrt{n / \log n}$, para alguma constante $K>0$ previamente escolhida. Então tem-se o seguinte

Teorema 3 Suponha que o modelo (4.1) seja válido com $e_{i}=e\left(t_{(i)}\right)$ sendo um ruído Gaussiano estacionário com média zero e covariâncias seguindo (4.2). Também, suponha que a ondaleta-mãe $\psi$ tenha r momentos nulos e possua suporte compacto. Então o estimador $\hat{f}$ da função $f$, dado por (4.3), atinge a menos de um fator logaritmico, a taxa de convergência ótima sobre uma grande quantidade de classes de Hölder $\Lambda^{\alpha}(M, B, 0)$ com $0<\alpha \leq r$. Isto é,

$$
\sup _{f \in \Lambda^{\alpha}(M)} E\|\hat{f}-f\|_{2}^{2} \leq C\left(\frac{\log n}{n}\right)^{2 \alpha /(2+2 \alpha)}
$$

$e$

$$
\sup _{f \in \Lambda^{\alpha}(M)} \frac{1}{n} \sum E\left\|\widehat{f\left(t_{k}\right)}-f\left(t_{k}\right)\right\|_{2}^{2} \leq C\left(\frac{\log n}{n}\right)^{2 \alpha /(2+2 \alpha)}
$$

para todo $M \in(0, \infty)$ e $\alpha \in(0, r]$.

Uma extensão desse teorema pode ser obtida supondo-se que a amostra provém de um esquema amostral de jittering. Assim, considere o modelo

$$
y_{i}=f\left(\frac{i}{n+1}+j_{i}\right)+e_{i} \quad i=1, \ldots, n,
$$

onde $j_{i}$ são variáveis aleatórias i.i.d. Uniforme $[-1 /(2(n+1)), 1 /(2(n+1))]$, para $i=1, \ldots, n$. Para o termo de erro, faça as mesmas suposições do Teorema 3. Considerando este modelo e o estimador $\hat{f}$ em (4.3), vale o seguinte

Corolário 1 Suponha que o modelo (4.4) seja válido com e $e_{i}$ sendo um ruído Gaussiano com média zero e covariâncias segundo (4.2). Também suponha que a ondaleta-mãe $\psi$ tenha $r$ momentos nulos e possua suporte compacto. Então para o estimador $\hat{f}$ em (4.3), os resultados do Teorema 3 permanecem válidos.

Note que esses resultados são fortemente baseados na distribuição Uniforme como geradora ou afetando os pontos amostrais. Na prática, usualmente escolhe-se a constante $K$ que faz $J^{\prime} \geq J$, e a aplicação de limiares aos coeficientes de ondaletas é realizado em todos os níveis a partir do nível $j_{0}$. 


\subsection{Simulações}

Um estudo de simulação foi conduzido para comparar o estimador baseado em amostras desigualmente espaçadas (com delineamento Uniforme) com o estimador baseado em amostras igualmente espaçadas. O pacote Wavethresh, implementado na linguagem $\mathrm{R}$, foi usado para todas as simulações.

Três funções $f(t)$ foram consideradas de teste, representando diferentes graus de variabilidade espacial.

1. Seno: $f(t)=\operatorname{sen}(2 \pi t)$.

2. HeaviSine: $f(t)=4 \operatorname{sen}(4 \pi t)-\operatorname{sgn}(t-0,3)-\operatorname{sgn}(0,72-t)$.

3. Doppler: $f(t)=\sqrt{t(1-t)} \operatorname{sen}(2 \pi(1+0,05) /(t+0,05))$.

As fórmulas para as duas últimas estão em Donoho e Johnstone (1994). As funções foram normalizadas para que tenham desvio padrão igual a 10. Um ruído estacionário gaussiano $\mathrm{AR}(1)$ com coeficiente $\phi=0,7$, foi adicionado a cada uma das funções. Como em Cai e Brown (1999), os estimadores foram comparados a dois níveis de ruído, um $\operatorname{com} \mathrm{SNR}=5$ e outro $\operatorname{com} \mathrm{SNR}=7$, onde

$$
S N R=\frac{(n-1)^{-1} \sum_{i=1}^{n}\left(f\left(t_{i}\right)-\bar{f}\right)^{2}}{\operatorname{Var}(\text { noise })},
$$

e $\bar{f}=n^{-1} \sum_{i=1}^{n} f\left(t_{i}\right)$. Amostras de tamanhos $n=256$ a 2048 foram consideradas.

A Tabela 4.1 relata os MSE sobre 200 replicações das funções de teste. A ondaleta usada foi a Symmlet 8 e os coeficientes foram limiarizados de modo suave a partir do nível $j_{0}$ indicado até a escala mais fina. O nível $j_{0}$ escolhido foi o nível do delineamento igualmente espaçado com menor MSE e os valores $\sigma_{j}$ foram estimados usando o desvio padrão amostral dos coeficientes de detalhes do nível $j$. Para construção do estimador (4.3), foi usada a constante $K=125$, que torna $J^{\prime} \geq J$ em todos os tamanhos amostrais usados. O teste t convencional foi usado para testar a significância das diferenças entre os MSE do delineamento aleatório e do delineamento igualmente espaçado. Quando a diferença não era significativa a $5 \%$, foi atribuído o valor zero.

A Tabela 4.1 mostra que o MSE dos delineamentos aleatórios é maior do que os do igualmente espaçado em quase todos os casos. O melhor ajuste foi alcançado para as funções seno enquanto o MSE é maior para as funções Doppler. Visualmente a reconstrução com delineamento uniforme é um pouco mais enrugado do que com o delineamento equiespaçado. Os casos relatados na Tabela 4.1, para $n=1024$ e SNR=7, são apresentados nas Figuras 4.1, 4.2 e 4.3 para as funções seno, HeaviSine e Doppler respectivamente. 
Tabela 4.1: MSE do estudo de simulação. As colunas com "\% dif." são as diferenças percentuais entre os MSE do delineamento aleatório e do delineamento igualmente espaçado. Foi atribuído o valor zero quando a diferença era insignificante a $5 \%$, de acordo com testes $t$ convencionais,

\begin{tabular}{|c|c|c|c|c|c|c|c|c|}
\hline \multirow[b]{2}{*}{$n$} & \multicolumn{4}{|c|}{$\mathrm{SNR}=5$} & \multicolumn{4}{|c|}{$\mathrm{SNR}=7$} \\
\hline & $j_{0}$ & Uniforme & Equiespaçado & $\%$ dif. & $j_{0}$ & Uniforme & Equiespaçado & $\%$ dif. \\
\hline \multicolumn{9}{|l|}{ Seno } \\
\hline 256 & 2 & 1,35 & 0,75 & 80 & 2 & 0,84 & 0,23 & 266 \\
\hline 512 & 2 & 0,69 & 0,41 & 69 & 2 & 0,48 & 0,15 & 222 \\
\hline 1024 & 2 & 0,39 & 0,23 & 71 & 3 & 0,15 & 0,09 & 58 \\
\hline 2048 & 2 & 0,22 & 0,14 & 54 & 3 & 0,08 & 0,05 & 58 \\
\hline \multicolumn{9}{|c|}{ HeaviSine } \\
\hline 256 & 3 & 3,61 & 2,32 & 55 & 4 & 1,63 & 1,33 & 22 \\
\hline 512 & 3 & 2,28 & 1,63 & 40 & 5 & 1,06 & 0,97 & 9 \\
\hline 1024 & 4 & 1,39 & 1,28 & 9 & 5 & 0,70 & 0,67 & 4 \\
\hline 2048 & 4 & 0,98 & 0,92 & 7 & 5 & 0,51 & 0,50 & 3 \\
\hline \multicolumn{9}{|c|}{ Doppler } \\
\hline 256 & 7 & 8,68 & 8,87 & 0 & 7 & 3,33 & 3,37 & 0 \\
\hline 512 & 7 & 7,76 & 7,40 & 5 & 8 & 2,43 & 2,22 & 9 \\
\hline 1024 & 6 & 7,08 & 5,67 & 25 & 8 & 2,14 & 1,84 & 16 \\
\hline 2048 & 8 & 4,98 & 4,71 & 6 & 8 & 1,67 & 1,41 & 18 \\
\hline
\end{tabular}

(a)

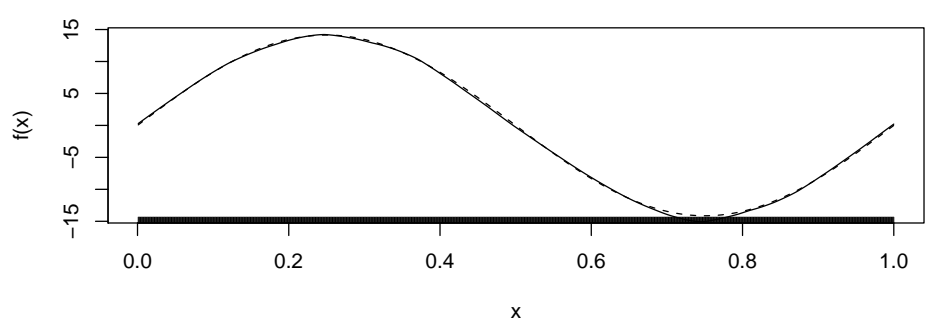

(b)

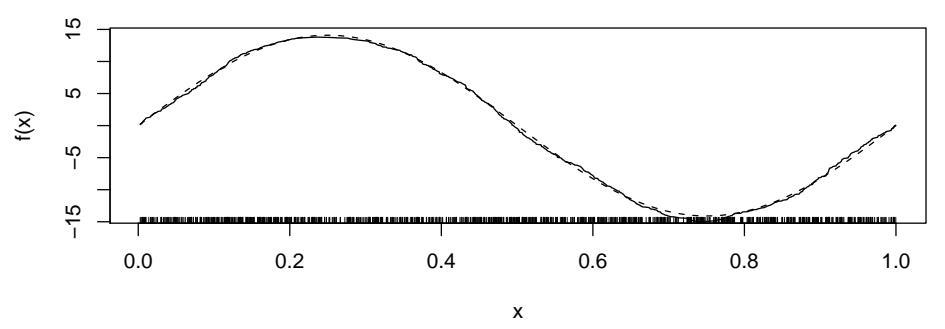

Figura 4.1: Funções seno (a) igualmente espaçada, (b) desigualmente espaçada (linhas tracejadas) e respectivas estimativas via ondaletas (linhas cheias) baseadas em $n=1024$ pontos e $\mathrm{SNR}=7$. Um ruído Gaussiano, seguindo um modelo $\operatorname{AR}(1)$ com coeficiente $\phi=0,7$, foi adicionado à função teste. A ondaleta Symmlet 8 foi usada com aplicação de limiares suaves por níveis a partir do nível $j_{0}=3$. 
(a)

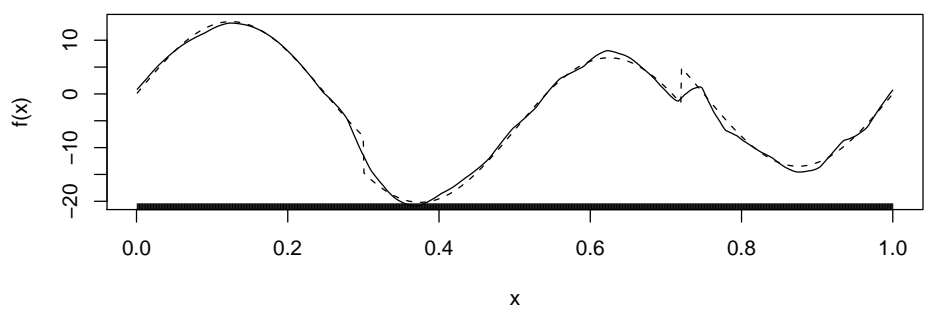

(b)

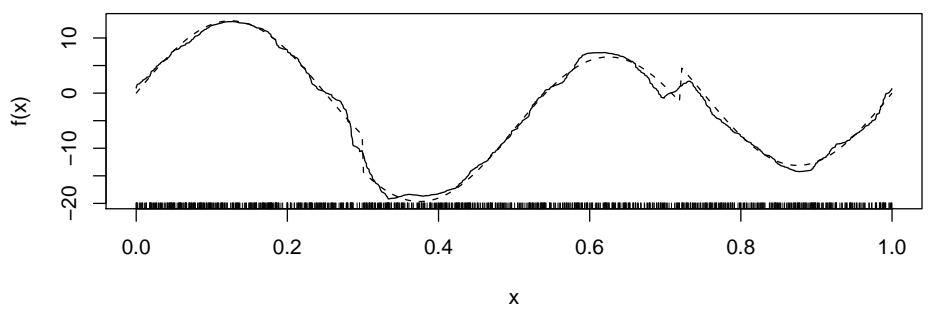

Figura 4.2: Funções HeaviSine (a) igualmente espaçada, (b) desigualmente espaçada (linhas tracejadas) e respectivas estimativas via ondaletas (linhas cheias) baseadas em $n=1024$ pontos e SNR=7. Um ruído Gaussiano, seguindo um modelo $\mathrm{AR}(1)$ com coeficiente $\phi=0,7$, foi adicionado à função teste. A ondaleta Symmlet 8 foi usada com aplicação de limiares suaves por níveis a partir do nível $j_{0}=5$.

(a)

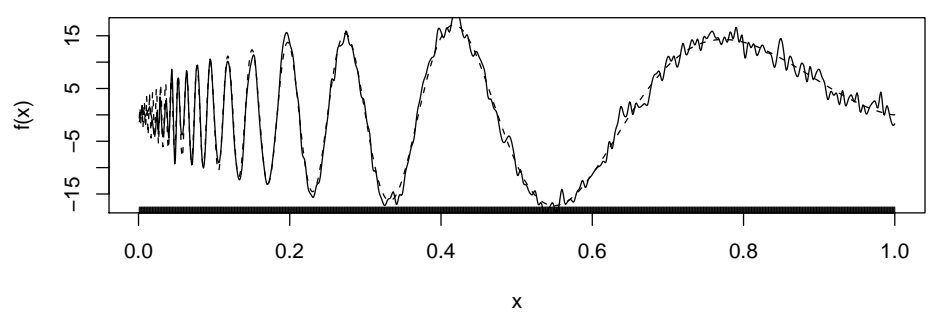

(b)

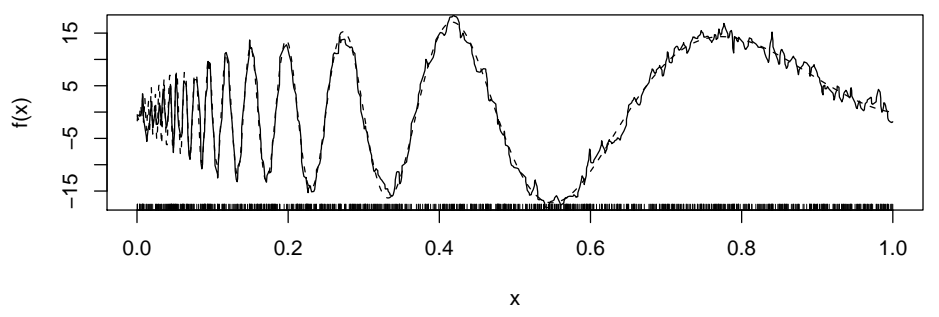

Figura 4.3: Funções Doppler (a) igualmente espaçada, (b) desigualmente espaçada (linhas tracejadas) e respectivas estimativas via ondaletas (linhas cheias) baseadas em $n=1024$ pontos e $\mathrm{SNR}=7$. Um ruído Gaussiano, seguindo um modelo $\operatorname{AR}(1)$ com coeficiente $\phi=0,7$, foi adicionado à função teste. A ondaleta Symmlet 8 foi usada com aplicação de limiares suaves por níveis a partir do nível $j_{0}=8$. 
(a)

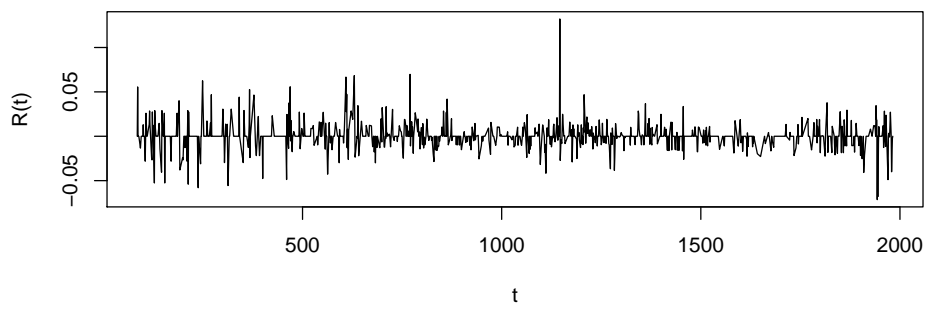

(b)

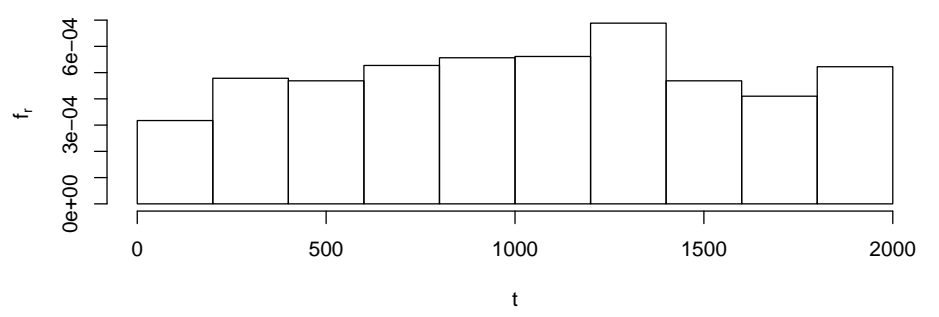

Figura 4.4: (a) Retornos simples $(R(t))$ de preços de fechamento das ações do Gouverneur Bancorp Inc. e (b) histograma dos dias em que eles ocorreram. De 9 de agosto de 1999 a 6 de fevereiro de 2007.

\subsection{Aplicação}

O delineamento Uniforme pode às vezes ser útil para estimar a tendência ou a volatilidade de ativos financeiros que não são negociados diariamente, ou que não são suficientemente negociados. Se para um período de tempo fixo, as negociações são uniformemente distribuídas neste intervalo, então pode-se aplicar limiares por níveis aos coeficientes de ondaletas.

Um desses casos é o preço de fechamento das ações do Gouverneur Bancorp Inc. de 9 de agosto de 1999 a 6 de fevereiro de 2007. Seus retornos simples e um histograma dos dias em que houve negociações são mostrados na Figura 4.4. Estes retornos não são diários pois as ações não foram negociadas diariamente no período. O teste de Kolmogorov-Smirnov não rejeita a hipótese de uma distribuição Uniforme $[0,1]$ para os dias, ao nível de $10 \%$ (p-valor=0,1334).

Os coeficientes empíricos de ondaletas dos retornos quadráticos são mostrados na Figura 4.5 juntamente com uma estimativa robusta da variância dos coeficientes de detalhes para cada um dos níveis de resolução considerados para aplicação de limiar. Sinais com erros correlacionados geram coeficientes de ondaletas com diferentes variâncias por nível (DONOHO; JOHNSTONE, 1994). Isto parece ser o caso aqui (como pode ser visto no gráfico de avaliação de autocorrelação) e, portanto, pode-se aplicar limiares dependentes do nível aos coeficientes. Os coeficientes após aplicação dos limiares suaves e a volatilidade estimada também são mostrados nessa figura.

Os retornos juntamente com os limites de \pm 2 vezes a volatilidade estimada são mostrados na Figura 4.6. Essa estimativa da volatilidade é muito enrugada e enfatiza alguns fatos estilizados 
(a)

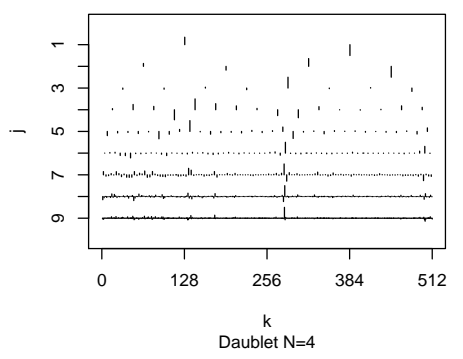

(c)

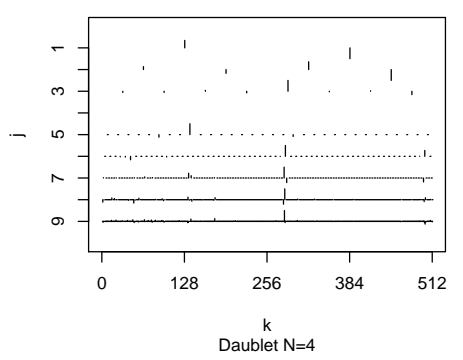

(b)

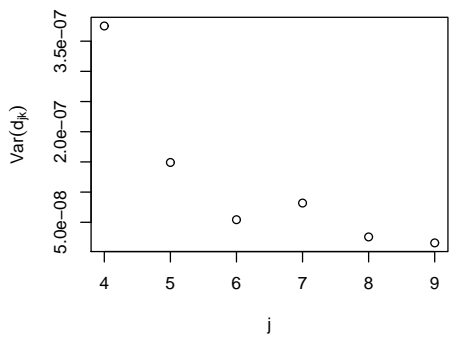

(d)

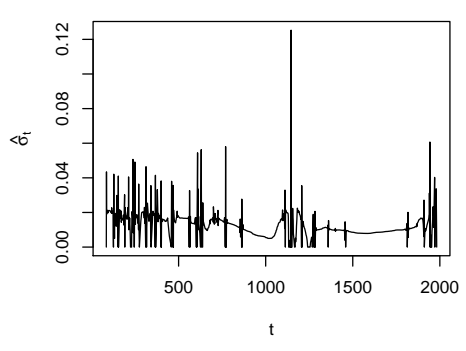

Figura 4.5: Gráficos de análise de ondaletas para os retornos simples dos preços de fechamento das ações do Gouverneur Bancorp Inc. da Figura 4.4: (a) Coeficientes empíricos da decomposição de ondaletas, segundo os níveis $j$ e os índices $k$; (b) verificação de correlação dos dados; (c) Coeficientes após aplicação de limiares suaves por níveis; (d) volatilidade estimada $\hat{\sigma}_{t}$.

conhecidos a respeito de retornos financeiros. Além disso, o percentual de retornos além dos limites corresponde a $7,7 \%$ dos dados, o que sugere que uma distribuição incondicional dos retornos com caudas mais pesadas do que as de uma distribuição normal.

\subsection{Conclusão}

Neste capítulo, consideram-se os casos especiais de amostras Uniforme e jittered, de um sinal na presença de erros estacionários Gaussianos com autocovariâncias absolutamente somáveis. Prova-se que nesses casos especiais, as amostras podem ser tratadas como se fossem igualmente espaçadas e com ruído correlacionado. Isto é, a uma transformada discreta de ondaletas, seguida da aplicação de limiares por níveis, nos fornece estimadores que adaptativamente atingem, a menos de um fator logarítmico, a taxa de convergência ótima em uma grande amplitude de classes de Hölder. Portanto, para amostras baseadas no delineamento Uniforme, há bons e rápidos algoritmos para o procedimento de estimação.

Um breve estudo de simulação foi realizado de modo a avaliar o desempenho numérico do método. Mostrou-se que o MSE é comparável ao obtido quando as amostras são igualmente espaçadas, como também ocorre quando os erros são i.i.d. (CAI; BROWN, 1999). Uma aplicação financeira ilustrou a utilidade do método. 


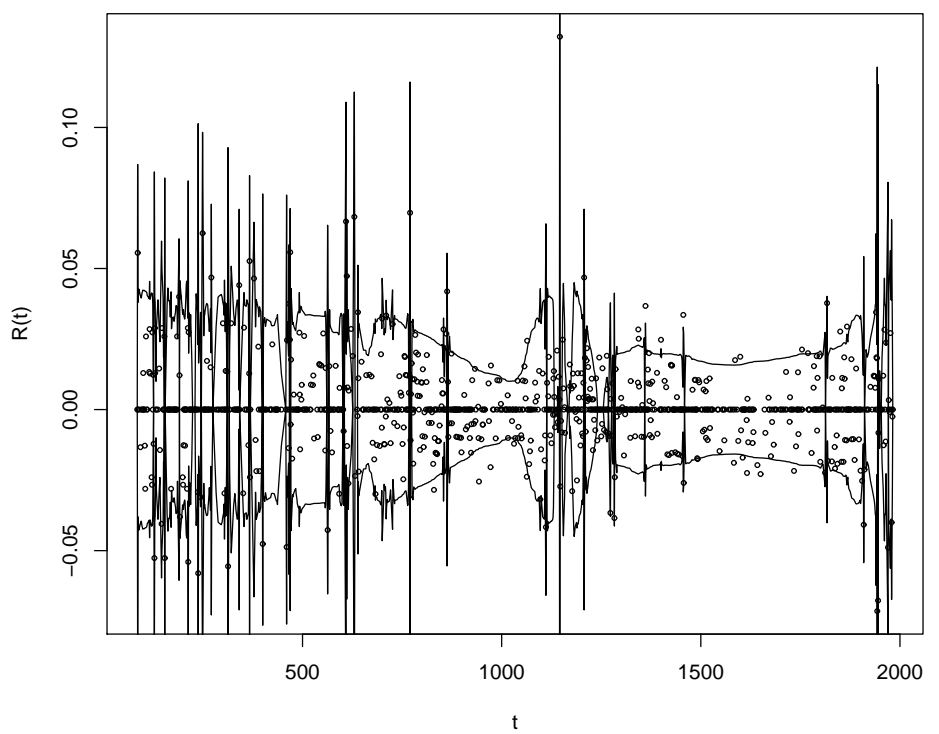

Figura 4.6: Retornos simples dos preços de fechamento das ações do Gouverneur Bancorp Inc. (círculos), juntamente com limites de \pm 2 vezes a volatilidade estimada por métodos de ondaletas (linhas).

\subsection{Provas}

\subsubsection{Prova da Proposição 2}

$\operatorname{Como} \operatorname{Cov}(e(r), e(s))=\sigma^{2} e^{-(n+1) \beta|r-s|}$, para algum $\beta>0,0<\sigma^{2}<\infty$ e $r$ e $s$ fixos, então,

$$
\begin{aligned}
\operatorname{Cov}\left(e\left(t_{(r)}\right), e\left(t_{(s)}\right)\right) & =E\left(E\left(e\left(t_{(r)}\right) e\left(t_{(s)}\right) \mid t_{(1)}, \ldots, t_{(n)}\right)\right)=E\left(\sigma^{2} e^{-(n+1) \beta\left|t_{(r)}-t_{(s)}\right|}\right) \\
& =E\left(\sigma^{2} \exp \left(-(n+1) \beta\left|\frac{r}{n+1}+j_{r}-\frac{s}{n+1}-j_{s}\right|\right)\right) \\
& =E\left(\sigma^{2} \exp \left(-(n+1) \beta\left|\frac{r-s}{n+1}+j_{r}-j_{s}\right|\right)\right)=\gamma(|r-s|) .
\end{aligned}
$$

Substituindo as variáveis aleatórias $j_{r}$ e $j_{s}$ pelos seus valores máximo e mínimo, respectivamente, essa expressão fica sendo menor ou igual a

$$
\begin{aligned}
E\left(\sigma^{2} \exp \left(-(n+1) \beta\left|\frac{r-s}{n+1}+\frac{2}{2(n+1)}\right|\right)\right) & =\sigma^{2} \exp \left(-(n+1) \beta\left|\frac{u+1}{n+1}\right|\right) \\
& =\sigma^{2} e^{-\beta|u+1|},
\end{aligned}
$$


onde $u=r-s$. Então,

$$
\lim _{n \rightarrow \infty} \sum_{u=-(n-1)}^{n-1}|\gamma(u)| \leq \sigma^{2} e^{-\beta} \lim _{n \rightarrow \infty} \sum_{u=-(n-1)}^{n-1} e^{-\beta u}<\infty .
$$

\subsubsection{Prova da Proposição 3}

Sejam $\left\{e\left(t_{(i)}\right), i=1, \ldots, n\right\}$ oriundos de um processo estocástico gaussiano $e(t), t \in(0,1)$, em tempo contínuo, de média zero, do tipo AR(1). Sejam os pontos aleatórios $0<t_{(1)}<\ldots<t_{(n)}<1$ tais que $t_{(i)} \sim \operatorname{Beta}(i, n-i+1)$. Seja $\operatorname{Cov}\left(e\left(t_{(i)}\right), e\left(t_{(j)}\right)\right)=\sigma^{2} e^{-(n+1) \beta\left|t_{(i)}-t_{(j)}\right|}$, para algum $\beta>0$, $0<\sigma^{2}<\infty$ e valores fixos $i$ e $j$.

Note que (JOHNSON; KOTZ; BALAKRISHNAN, 1995, p. 217):

$$
E\left(\left(t_{(i)}-t_{(j)}\right)^{k}\right)=\frac{\Gamma(|i-j|+k) \Gamma(n+1)}{\Gamma(|i-j|) \Gamma(n+1+k)}=\frac{(|i-j|+k-1) ! n !}{(|i-j|-1) !(n+k) !} .
$$

Então,

$$
\begin{aligned}
\operatorname{Cov}\left(e\left(t_{(i)}\right), e\left(t_{(j)}\right)\right) & =E\left(E\left(e\left(t_{(i)}\right) e\left(t_{(j)}\right) \mid t_{(1)}, \ldots, t_{(n)}\right)\right)=E\left(\sigma^{2} e^{-(n+1) \beta\left|t_{(i)}-t_{(j)}\right|}\right) \\
& =\sigma^{2} \sum_{k=0}^{\infty}(-1)^{k} \frac{(n+1)^{k} \beta^{k}}{k !} E\left(\left|t_{(i)}-t_{(j)}\right|^{k}\right) \\
& =\sigma^{2} \sum_{k=0}^{\infty}(-1)^{k} \frac{(n+1)^{k} \beta^{k}}{k !} \frac{(|i-j|+k-1) ! n !}{(|i-j|-1) !(n+k) !}=\gamma(|i-j|) .
\end{aligned}
$$

Como os momentos de $t_{(i)}-t_{(j)}$ são finitos, o Teorema da Convergência Dominada permite justificar a troca de ordem do somatório infinito e da esperança matemática.

Para avaliar $\lim _{n \rightarrow \infty} \sum_{u=1}^{n-1}|\gamma(u)|$, primeiro note que

$$
\begin{aligned}
|\gamma(|i-j|)| & =\left|E\left(\sigma^{2} e^{-(n+1) \beta\left|t_{(i)}-t_{(j)}\right|}\right)\right| \\
& =E\left(\sigma^{2} e^{-(n+1) \beta\left|t_{(i)}-t_{(j)}\right|}\right)=\gamma(|i-j|) .
\end{aligned}
$$

Note também que

$$
\begin{aligned}
\sum_{u=1}^{n-1} \frac{(u+k-1) !}{(u-1) !} & =\sum_{v=0}^{n-2} \frac{(v+k) !}{v !} \\
& =k ! \sum_{v=0}^{n-2} \frac{(v+k) !}{v ! k !} \\
& =k !\left(\begin{array}{c}
k+(n-2)+1 \\
k+1
\end{array}\right)
\end{aligned}
$$




$$
\begin{aligned}
& =\frac{k !(n+k-1) !}{(k+1) !(n-2) !} \\
& =\frac{(n+k-1) !}{(k+1)(n-2) !},
\end{aligned}
$$

onde (4.5) usa o resultado 0.15.1 em Gradshteǐn et al. (2007). Então, usando esses fatos,

$$
\begin{aligned}
\sum_{u=1}^{n-1}|\gamma(u)|=\sum_{u=1}^{n-1} \gamma(u) & =\sum_{u=1}^{n-1} \sigma^{2} \sum_{k=0}^{\infty}(-1)^{k} \frac{(n+1)^{k} \beta^{k}}{k !} \frac{(u+k-1) ! n !}{(u-1) !(n+k) !} \\
& =\sigma^{2} \sum_{k=0}^{\infty}(-1)^{k} \frac{(n+1)^{k} \beta^{k} n !}{k !(n+k) !} \sum_{u=1}^{n-1} \frac{(u+k-1) !}{(u-1) !} \\
& =\sigma^{2} \sum_{k=0}^{\infty}(-1)^{k} \frac{(n+1)^{k} \beta^{k} n !}{k !(n+k) !} \frac{(n+k-1) !}{(k+1)(n-2) !} \\
& =\sigma^{2} \sum_{k=0}^{\infty}(-1)^{k} \frac{(n+1)^{k} \beta^{k}}{k !} \frac{n(n-1)}{(n+k)(k+1)} .
\end{aligned}
$$

Uma justificativa para a troca de ordem dos somatórios de (4.6) para (4.7), é a seguinte. Em (4.6), seja

$$
g_{k}(u)=\sigma^{2}(-1)^{k} \frac{(n+1)^{k} \beta^{k}}{k !} \frac{(u+k-1) ! n !}{(u-1) !(n+k) !},
$$

e note que

$$
\begin{aligned}
\left|g_{k}(u)\right| & =\sigma^{2} \frac{(n+1)^{k} \beta^{k}}{k !} \frac{(u+k-1) ! n !}{(u-1) !(n+k) !} \\
& <\sum_{k=0}^{\infty} \sigma^{2} \frac{(n+1)^{k} \beta^{k}}{k !} \frac{(u+k-1) ! n !}{(u-1) !(n+k) !} \\
& =\sigma^{2} \sum_{k=0}^{\infty} \frac{(n+1)^{k} \beta^{k}}{k !} \frac{(|i-(i+u)|+k-1) ! n !}{(|i-(i+u)|-1) !(n+k) !} \\
& =\sigma^{2} \sum_{k=0}^{\infty} \frac{(n+1)^{k} \beta^{k}}{k !} E\left(\left|t_{(i)}-t_{(i+u)}\right|^{k}\right) \\
& =E\left(\sigma^{2} e^{(n+1) \beta\left|t_{(i)}-t_{(i+u)}\right|}\right) \\
& \leq \sigma^{2} e^{(n+1) \beta}
\end{aligned}
$$

para $u=1, \ldots, n-1$, e para todo $k \geq 0$. Seja $u$ a medida de contagem tal que

$$
\begin{aligned}
\sum_{u=1}^{n-1} \sum_{k=0}^{\infty} g_{k}(u) & =\sum_{u=1}^{n-1} \lim _{\kappa \rightarrow \infty} \sum_{k=0}^{\kappa} g_{k}(u) \\
& =\int_{1}^{n-1} \lim _{\kappa \rightarrow \infty} \sum_{k=0}^{\kappa} g_{k}(u) d u
\end{aligned}
$$




$$
\begin{aligned}
& =\lim _{\kappa \rightarrow \infty} \int_{1}^{n-1} \sum_{k=0}^{\kappa} g_{k}(u) d u \\
& =\lim _{\kappa \rightarrow \infty} \sum_{k=0}^{\kappa} \int_{1}^{n-1} g_{k}(u) d u \\
& =\sum_{k=0}^{\infty} \sum_{u=1}^{n-1} g_{k}(u),
\end{aligned}
$$

onde o Teorema da Convergência Dominada foi usado em (4.9).

Avaliando o somatório em (4.8), tem-se que

$$
\begin{aligned}
\sum_{k=0}^{\infty} & \frac{(-1)^{k}(n+1)^{k} \beta^{k}}{k !} \frac{1}{(n+k)(k+1)}= \\
& =\sum_{k=0}^{\infty} \frac{(-1)^{k}(n+1)^{k} \beta^{k}}{k !} \frac{\Gamma(n+k)}{\Gamma(n+k+1)} \frac{\Gamma(k+1)}{\Gamma(k+2)} \\
& =\sum_{k=0}^{\infty} \frac{(-1)^{k}(n+1)^{k} \beta^{k}}{k !} \frac{\frac{\Gamma(n+k)}{\Gamma(n)} \Gamma(n)}{\frac{\Gamma(n+k+1)}{\Gamma(n+1)} \Gamma(n+1)} \frac{\frac{\Gamma(k+1)}{\Gamma(1)} \Gamma(1)}{\frac{\Gamma(k+2)}{\Gamma(2)} \Gamma(2)} \\
& =\sum_{k=0}^{\infty} \frac{(-1)^{k}(n+1)^{k} \beta^{k}}{k !} \frac{(n)_{k}(1)_{k}}{(n+1)_{k}(2)_{k}} \frac{1}{n} \\
& =\frac{1}{n}{ }_{2} F_{2}(n, 1 ; n+1,2 ;(-1)(n+1) \beta),
\end{aligned}
$$

onde $\Gamma(n)$ denota a função gama, o símbolo de Pochhammer $(a)_{k}=\Gamma(a+k) / \Gamma(a)$, e ${ }_{2} F_{2}(a, b ; c, d ; z)$ denota uma função hipergeométrica generalizada.

Denotando a funcão hipergeométrica confluente do primeiro tipo por ${ }_{1} F_{1}(a, b, z)$, tem-se que (http://functions.wolfram.com/07.25.03.0005.01)

$$
\begin{aligned}
& \frac{1}{b-a}\left(b_{1} F_{1}(a, a+1, z)-a{ }_{1} F_{1}(b, b+1, z)\right) \\
& =\frac{1}{b-a}\left(b \sum_{k=0}^{\infty} \frac{(a)_{k}}{(a+1)_{k}} \frac{z^{k}}{k !}-a \sum_{k=0}^{\infty} \frac{(b)_{k}}{(b+1)_{k}} \frac{z^{k}}{k !}\right) \\
& =\frac{1}{b-a}\left(b \sum_{k=0}^{\infty} \frac{b+k}{b+k} \frac{(a)_{k}}{(a+1)_{k}} \frac{z^{k}}{k !}-a \sum_{k=0}^{\infty} \frac{a+k}{a+k} \frac{(b)_{k}}{(b+1)_{k}} \frac{z^{k}}{k !}\right) \\
& =\frac{1}{b-a}\left(\sum_{k=0}^{\infty} \frac{(b+k)(b)_{k}}{(b+1)_{k}} \frac{(a)_{k}}{(a+1)_{k}} \frac{z^{k}}{k !}-\sum_{k=0}^{\infty} \frac{(a+k)(a)_{k}}{(a+1)_{k}} \frac{(b)_{k}}{(b+1)_{k}} \frac{z^{k}}{k !}\right) \\
& =\frac{1}{b-a}\left(b \sum_{k=0}^{\infty} \frac{(b)_{k}}{(b+1)_{k}} \frac{(a)_{k}}{(a+1)_{k}} \frac{z^{k}}{k !}-a \sum_{k=0}^{\infty} \frac{(a)_{k}}{(a+1)_{k}} \frac{(b)_{k}}{(b+1)_{k}} \frac{z^{k}}{k !}\right)
\end{aligned}
$$




$$
\begin{aligned}
& =\frac{b-a}{b-a} \sum_{k=0}^{\infty} \frac{(b)_{k}}{(b+1)_{k}} \frac{(a)_{k}}{(a+1)_{k}} \frac{z^{k}}{k !} \\
& ={ }_{2} F_{2}(a, b ; a+1, b+1 ; z),
\end{aligned}
$$

e aplicando este resultado à equação (4.10),

$$
\begin{aligned}
& \frac{1}{n}{ }_{2} F_{2}(n, 1 ; n+1,2 ;(-1)(n+1) \beta)= \\
& \quad=\frac{1}{n} \frac{1}{1-n}\left({ }_{1} F_{1}(n, n+1,(-1)(n+1) \beta)-n_{1} F_{1}(1,2,(-1)(n+1) \beta)\right) .
\end{aligned}
$$

Pelo resultado 9.236.4 em Gradshteřn et al. (2007), aplicando

$$
{ }_{1} F_{1}(a, a+1, z)=a(-z)^{-a}(\Gamma(a)-\Gamma(a,-z))
$$

à última expressão, tem-se que

$$
\begin{aligned}
\sum_{k=0}^{\infty} & \frac{(-1)^{k}(n+1)^{k} \beta^{k}}{k !} \frac{1}{(n+k)(k+1)}= \\
= & \frac{1}{n} \frac{1}{1-n}\left(\frac{n}{[(n+1) \beta]^{n}}[\Gamma(n)-\Gamma(n,(n+1) \beta)]-n_{1} F_{1}(1,2,(-1)(n+1) \beta)\right) \\
= & \frac{1}{(n+1)(n-1)}\left(\frac{1}{[(n+1) \beta]^{n}}[-(n+1) \Gamma(n)+(n+1) \Gamma(n,(n+1) \beta)]\right) \\
& \quad+\frac{1 F_{1}(1,2,(-1)(n+1) \beta)}{n-1}
\end{aligned}
$$

onde $\Gamma(n, a)=\int_{a}^{\infty} t^{n-1} e^{-t} d t$ denota a função gama incompleta. Usando (4.11) também tem-se que

$$
\begin{aligned}
\frac{{ }_{1} F_{1}(1,2,(-1)(n+1) \beta)}{n-1} & =\frac{1}{(n+1) \beta(n-1)}[\Gamma(1)-\Gamma(1, \beta(n+1))] \\
& =\frac{1}{\beta\left(n^{2}-1\right)}\left[1-\int_{\beta(n+1)}^{\infty} t^{1-1} e^{-t} d t\right] \\
& =\frac{1}{\beta\left(n^{2}-1\right)}\left[1-e^{-\beta(n+1)}\right] .
\end{aligned}
$$

Logo, para $n>1$,

$$
\begin{aligned}
\sum_{k=0}^{\infty}(-1)^{k} \frac{(n+1)^{k} \beta^{k}}{k !(n+k)(k+1)}= & \frac{[-(n+1) \Gamma(n)+(n+1) \Gamma(n, \beta(n+1))]}{\left(n^{2}-1\right)[\beta(n+1)]^{n}} \\
& +\frac{1-e^{-\beta(n+1)}}{\beta\left(n^{2}-1\right)}
\end{aligned}
$$


onde a função gama incompleta

$$
\begin{aligned}
\Gamma(n, \beta(n+1)) & =\int_{\beta(n+1)}^{\infty} t^{n-1} e^{-t} d t \\
& =(n-1) ! e^{-\beta(n+1)} \sum_{k=0}^{n-1} \beta^{k}(n+1)^{k} / k ! \\
& \leq(n-1) ! e^{-\beta(n+1)} \sum_{k=0}^{\infty} \beta^{k}(n+1)^{k} / k ! \\
& =(n-1) ! e^{-\beta(n+1)} e^{\beta(n+1)} \\
& =(n-1) !=\Gamma(n),
\end{aligned}
$$

quando $n$ é um inteiro. Aplicando estes resultados em (4.8), tem-se que para todo $n>1$,

$$
\begin{aligned}
\sum_{u=1}^{n-1}|\gamma(u)|= & \sigma^{2}\left\{\frac{n(n-1)[-(n+1) \Gamma(n)+(n+1) \Gamma(n, \beta(n+1))]}{\left(n^{2}-1\right)[\beta(n+1)]^{n}}\right. \\
& \left.+\frac{n}{\beta(n+1)}-\frac{n(n-1) e^{-\beta(n+1)}}{\beta\left(n^{2}-1\right)}\right\} \\
\leq & \sigma^{2}\left\{\frac{n(n-1)[-(n+1) \Gamma(n)+(n+1) \Gamma(n)]}{\left(n^{2}-1\right)[\beta(n+1)]^{n}}+\frac{1}{\beta}\right\} \\
= & \frac{\sigma^{2}}{\beta} .
\end{aligned}
$$

Portanto, $\lim _{n \rightarrow \infty} \sum_{u=1}^{n-1}|\gamma(u)| \leq \sigma^{2} / \beta<\infty$.

\subsubsection{Prova do Teorema 3}

Seja $y_{i}=f\left(t_{(i)}\right)+e_{i}$, onde $e_{1}, \ldots, e_{n}$ provêm de um processo estacionário gaussiano com $E\left(e_{i}\right)=0$, $\operatorname{Var}\left(e_{i}\right)=\sigma^{2}$ e $\operatorname{Cov}\left(e_{r}, e_{s}\right)=\gamma(|r-s|)$, para $i, r, s=1, \ldots, n$. Suponha que $\sum_{u=-\infty}^{\infty}|\gamma(u)|<\infty$. Seja $t_{i} \sim U[0,1]$ e $t_{(1)}<\ldots<t_{(n)}$ as respectivas estatísticas de ordem. Então, $t_{(i)} \sim \operatorname{Beta}(i, n-i+1)$, e

$$
\operatorname{Var}\left(t_{(i)}\right)=\frac{(n+1) i-i^{2}}{(n+1)^{2}(n+2)}
$$

como no Lema 2 de Cai e Brown (1999). Observe que o Lema 3 de Cai e Brown (1999) também continua válido, porque não é afetado pela correlação dos erros.

Daqui em diante, $C_{1}, C_{2}, \ldots, C_{18}$ denotarão constantes positivas que não dependem de $n$. Seja

$$
\tilde{f}(x)=\sum_{i=0}^{n-1} n^{-1 / 2} y_{i+1} \phi_{J, i}(x) ;
$$




$$
\begin{aligned}
f_{n}(x) & =\sum_{i=0}^{n-1} n^{-1 / 2} f\left(\frac{i+1}{n+1}\right) \phi_{J, i}(x) ; \\
f(x) & =\sum_{k=0}^{n-1} c_{J, k} \phi_{J, k}(x)+\sum_{j=J}^{\infty} \sum_{k=0}^{2^{j}-1} d_{j, k} \psi_{j, k}(x),
\end{aligned}
$$

onde $k \in \mathbb{Z}, c_{J, k}=\left\langle f, \phi_{J, k}\right\rangle, d_{j, k}=\left\langle f, \psi_{j, k}\right\rangle, n=2^{J}, f \in \Lambda^{\alpha}(M, B, 0)$, com $\alpha>0$.

Reescreva

$$
\begin{aligned}
\tilde{f}(x) & =f(x)+\left[f_{n}(x)-f(x)\right]+\left[\tilde{f}(x)-f_{n}(x)\right] \\
& =f(x)+\left[f_{n}(x)-f(x)\right]+\left[\sum_{i=0}^{n-1} n^{-1 / 2}\left(f\left(t_{(i+1)}\right)+e_{i+1}\right) \phi_{J, i}(x)-f_{n}(x)\right] \\
& =f(x)+A(x)+B(x)+R(x),
\end{aligned}
$$

onde

$$
\begin{aligned}
& A(x)=f_{n}(x)-f(x) \\
& B(x)=\sum_{i=0}^{n-1} n^{-1 / 2} f\left(t_{(i+1)}\right) \phi_{J, i}(x)-f_{n}(x) \\
& R(x)=\sum_{i=0}^{n-1} n^{-1 / 2} e_{i+1} \phi_{J, i}(x) .
\end{aligned}
$$

Note que $A(x)$ não é aleatório enquanto $B(x)$ é aleatório, mas depende apenas de $\left\{t_{i}\right\}_{i=1}^{n}$. Para algum $j_{0} \in \mathbb{Z}$ e alguma base de ondaletas de suporte compacto $\left\{\phi_{j_{0}, k}, k \in \mathbb{Z}\right\} \cup\left\{\psi_{j, k}, j \geq j_{0}, k \in \mathbb{Z}\right\}, \psi$ com $r \geq \alpha$ momentos nulos, seja

$$
\begin{gathered}
c_{j_{0}, k}=\left\langle f, \phi_{j_{0}, k}\right\rangle, \quad \tilde{a}_{j_{0}, k}=\left\langle A, \phi_{j_{0}, k}\right\rangle, \quad \tilde{b}_{j_{0}, k}=\left\langle B, \phi_{j_{0}, k}\right\rangle, \quad \tilde{r}_{j_{0}, k}=\left\langle R, \phi_{j_{0}, k}\right\rangle ; \\
\tilde{c}_{j_{0}, k}=c_{j_{0}, k}+\tilde{a}_{j_{0}, k}+\tilde{b}_{j_{0}, k}+\tilde{r}_{j_{0}, k}=\int_{0}^{1} \tilde{f}(x) \phi_{j_{0}, k}(x) d x ; \\
d_{j, k}=\left\langle f, \psi_{j, k}\right\rangle, \quad a_{j, k}=\left\langle A, \psi_{j, k}\right\rangle, \quad b_{j, k}=\left\langle B, \psi_{j, k}\right\rangle, \quad r_{j, k}=\left\langle R, \psi_{j, k}\right\rangle ; \\
\tilde{d}_{j, k}=d_{j, k}^{\prime}+r_{j, k}, \text { onde } d_{j, k}^{\prime}=d_{j, k}+a_{j, k}+b_{j, k} .
\end{gathered}
$$

Esta base de ondaletas pode ser diferente da usada nas equações (4.12), (4.13) e (4.14). Naquelas equações, será usada a função escala de Haar

$$
\phi_{J, i}(x)=2^{J / 2} \phi\left(2^{J} x-i\right)=\sqrt{n} I((n x-i) \in(0,1]), \quad i=0, \ldots, n-1,
$$


onde $I(\cdot)$ denota a usual função indicadora. Então, para $k=1, \ldots, n$,

$$
\tilde{f}(k / n)=\sum_{i=0}^{n-1} y_{i+1} / \sqrt{n} \phi_{J, i}(k / n)=\sum_{i=0}^{n-1} y_{i+1} I((n k / n-i) \in(0,1])=y_{k},
$$

tal que $\tilde{f}(x)$ será, doravante, uma aproximação constante por partes de $f(x)$, baseada nos pontos observados $y_{1}, \ldots, y_{n}$. Similarmente, teremos

$$
f_{n}\left(\frac{k}{n}\right)=f\left(\frac{k}{n+1}\right), \quad R\left(\frac{k}{n}\right)=e_{k} .
$$

Também sejam

$$
\tilde{r}_{j_{0}, k}=\frac{1}{n} \sum_{i=1}^{n} e_{i} \phi_{j_{0}, k}(i / n) \quad \text { and } \quad \hat{r}_{j, k}=\frac{1}{n} \sum_{i=1}^{n} e_{i} \psi_{j, k}(i / n)
$$

estimadores de $\tilde{r}_{j_{0}, k}$ e $r_{j, k}$, respectivamente, como em Morettin (1999). Seja

$$
\begin{gathered}
\hat{c}_{j_{0}, k}=c_{j_{0}, k}+\tilde{a}_{j_{0}, k}+\tilde{b}_{j_{0}, k}+\tilde{r}_{j_{0}, k} \\
\tilde{d}_{j, k}=d_{j, k}^{\prime}+\hat{r}_{j, k}, \quad \hat{d}_{j, k}=\operatorname{sgn}\left(\tilde{d}_{j, k}\right)\left(\left|\tilde{d}_{j, k}-\lambda\right|\right)_{+},
\end{gathered}
$$

onde $\lambda=\sigma_{j, k} \sqrt{2 n^{-1} \log n}, n^{-1} \sigma_{j, k}^{2}=\operatorname{Var}\left(\hat{r}_{j, k}\right) \mathrm{e}$

$$
\begin{aligned}
\operatorname{Var}\left(\hat{r}_{j, k}\right) & =\frac{1}{n^{2}} \operatorname{Cov}\left(\sum_{i=1}^{n} e_{i} \psi_{j, k}(i / n), \sum_{t=1}^{n} e_{t} \psi_{j, k}(t / n)\right) \\
& =\frac{1}{n^{2}} \sum_{i=1}^{n} \sum_{t=1}^{n} \psi_{j, k}(i / n) \psi_{j, k}(t / n) \operatorname{Cov}\left(e_{i}, e_{t}\right) \\
& \leq C_{1}\|\psi\|_{\infty}^{2} \frac{2^{j}}{n^{2}} \sum_{i=1}^{n} \sum_{t=1}^{n}|\gamma(i-t)| \\
& =C_{1}\|\psi\|_{\infty}^{2} \frac{2^{j}}{n^{2}} \sum_{u=-(n-1)}^{n-1}|\gamma(u)|(n-|u|) \\
& \leq C_{1}\|\psi\|_{\infty}^{2} \frac{2^{j}}{n} \sum_{u=-(n-1)}^{n-1}|\gamma(u)| \\
& \leq C_{2} 2^{j} n^{-1} .
\end{aligned}
$$

Por argumento análogo $\operatorname{Var}\left(\tilde{r}_{j_{0}, k}\right) \leq C_{3} 2^{j_{0}} n^{-1}$. Observe que $\tilde{d}_{j, k} \sim N\left(d_{j, k}^{\prime}, n^{-1} \sigma_{j, k}^{2}\right)$.

Agora seja $\hat{f}(x)$ um estimador de $f(x)$ para todo $x \in[0,1]$, onde

$$
\hat{f}(x)=\sum_{k=0}^{2^{j_{0}}-1} \hat{c}_{j_{0}, k} \phi_{j_{0}, k}(x)+\sum_{j=j_{0}}^{J^{\prime}-1} \sum_{k=0}^{2^{j}-1} \hat{d}_{j, k} \psi_{j, k}(x),
$$


e $J^{\prime}$ é o maior inteiro em que $2^{J^{\prime}} \leq K \sqrt{n / \log n}$. Então, a função risco é

$$
\begin{aligned}
E\left(\|\hat{f}-f\|_{2}^{2}\right)= & E\left(\int_{0}^{1}[\hat{f}(x)-f(x)]^{2} d x\right) \\
= & E\left(\int _ { 0 } ^ { 1 } \left[\sum_{k=0}^{2^{j_{0}}-1} \hat{c}_{j_{0}, k} \phi_{j_{0}, k}(x)+\sum_{j=j_{0}} \sum_{k=0}^{J^{\prime}-1} \hat{d}_{j, k} \psi_{j, k}(x)\right.\right. \\
& -\sum_{k=0}^{2^{j_{0}}-1} c_{j_{0}, k} \phi_{j_{0}, k}(x)-\sum_{j=j_{0}}^{J^{\prime}-1} \sum_{k=0}^{2^{j}-1} d_{j, k} \psi_{j, k}(x) \\
& \left.\left.-\sum_{j=J^{\prime}}^{\infty} \sum_{k=0}^{2^{j}-1} d_{j, k} \psi_{j, k}(x)\right]^{d x} d x\right) \\
= & \left(\int _ { 0 } ^ { 1 } \left[\sum_{k=0}^{2^{j_{0}-1}}\left(\hat{c}_{j_{0}, k}-c_{j_{0}, k}\right) \phi_{j_{0}, k}(x)\right.\right. \\
& +\sum_{j=j_{0}}^{J^{\prime}-1} \sum_{k=0}^{2^{j}-1}\left(\hat{d}_{j, k}-d_{j, k}\right) \psi_{j, k}(x) \\
& \left.\left.-\sum_{j=J^{\prime}}^{\infty} \sum_{k=0}^{2^{j}-1} d_{j, k} \psi_{j, k}(x)\right]^{2} d x\right)
\end{aligned}
$$

Pela ortogonalidade da base de ondaletas, esta expressão é igual a

$$
\begin{gathered}
E\left(\sum_{k=0}^{2^{j_{0}-1}} \int_{0}^{1}\left(\hat{c}_{j_{0}, k}-c_{j_{0}, k}\right)^{2} \phi_{j_{0}, k}^{2}(x) d x+\sum_{j=j_{0}}^{J^{\prime}-1} \sum_{k=0}^{2^{j}-1} \int_{0}^{1}\left(\hat{d}_{j, k}-d_{j, k}\right)^{2} \psi_{j, k}^{2}(x) d x\right. \\
\left.+\sum_{j=J^{\prime}}^{\infty} \sum_{k=0}^{2^{j}-1} \int_{0}^{1} d_{j, k}^{2} \psi_{j, k}^{2}(x) d x\right)
\end{gathered}
$$

e pela ortonormalidade da base de ondaletas

$$
E\left(\|\hat{f}-f\|_{2}^{2}\right)=\sum_{k=0}^{2^{j} 0-1} E\left(\left(\hat{c}_{j_{0}, k}-c_{j_{0}, k}\right)^{2}\right)+\sum_{j=j_{0}}^{J^{\prime}-1} \sum_{k=0}^{2^{j}-1} E\left(\left(\hat{d}_{j, k}-d_{j, k}\right)^{2}\right)+\sum_{j=J^{\prime}}^{\infty} \sum_{k=0}^{2^{j}-1} d_{j, k}^{2}
$$

Pelo Teorema 2.9.1 de Daubechies (1992) (veja também o Lema 1 de Cai e Brown (1999)),

$$
\sum_{j=J^{\prime}}^{\infty} \sum_{k=0}^{2^{j}-1} d_{j, k}^{2} \leq C_{4} \sum_{j=J^{\prime}}^{\infty} 2^{j} 2^{2(-j(1 / 2+\alpha))}=C_{4} \sum_{j=J^{\prime}}^{\infty} 2^{-2 j \alpha}
$$




$$
\begin{aligned}
& =C_{4}\left(\sum_{j=0}^{\infty} 2^{-2 j \alpha}-\sum_{j=0}^{J^{\prime}-1} 2^{-2 j \alpha}\right) \\
& =C_{4} \frac{\left(2^{-2 \alpha}\right)^{J^{\prime}}}{1-2^{-2 \alpha}} \leq C_{4}\left(2^{J^{\prime}}\right)^{-2 \alpha} \\
& \leq C_{4}\left(2^{J^{\prime}+1-1}\right)^{-2 \alpha}=C_{5}\left(2^{J^{\prime}+1}\right)^{-2 \alpha} \\
& \leq C_{5}\left(K^{2} \frac{n}{\log n}\right)^{-2 \alpha / 2}=C_{5}\left(K^{2} \frac{\log n}{n}\right)^{\alpha} \\
& \leq C_{5} K^{2 \alpha}\left(\frac{\log n}{n}\right)^{\frac{2 \alpha}{2+2 \alpha}}, \quad \forall \alpha>0, n \geq 3 .
\end{aligned}
$$

Doravante, será usada repetidamente uma específica aplicação da desigualdade (numérica) de Hölder: $(a+b)^{2} \leq 2 a^{2}+2 b^{2}, a, b \in \mathbb{R}$. Também, seja $E_{1}(Y)=E\left(Y \mid t_{(1)}, \ldots, t_{(n)}\right)$ para qualquer variável aleatória $Y$.

Temos

$$
\begin{aligned}
E\left(\left(\hat{c}_{j_{0}, k}-c_{j_{0}, k}\right)^{2}\right) & =E\left(E_{1}\left(\left(\hat{c}_{j_{0}, k}-c_{j_{0}, k}\right)^{2}\right)\right) \\
& =E\left(E_{1}\left(\left(\tilde{r}_{j_{0}, k}+\tilde{a}_{j_{0}, k}+\tilde{b}_{j_{0}, k}\right)^{2}\right)\right) \\
& =E\left(\tilde{r}_{j_{0}, k}^{2}\right)+0+E\left(E_{1}\left(\left(\tilde{a}_{j_{0}, k}+\tilde{b}_{j_{0}, k}\right)^{2}\right)\right) \\
& \leq E\left(\tilde{r}_{j_{0}, k}{ }^{2}\right)+E\left(E_{1}\left(2 \tilde{a}_{j_{0}, k}^{2}+2 \tilde{b}_{j_{0}, k}^{2}\right)\right) \\
& =E\left(\tilde{r}_{j_{0}, k}{ }^{2}\right)+2 \tilde{a}_{j_{0}, k}^{2}+2 E\left(\tilde{b}_{j_{0}, k}^{2}\right) \\
& \leq C_{3} 2^{j_{0}} n^{-1}+2 \tilde{a}_{j_{0}, k}^{2}+2 E\left(\tilde{b}_{j_{0}, k}^{2}\right),
\end{aligned}
$$

e então,

$$
\sum_{k=0}^{2^{j_{0}}-1} E\left(\left(\hat{c}_{j_{0}, k}-c_{j_{0}, k}\right)^{2}\right) \leq C_{3} 2^{2 j_{0}} n^{-1}+2 \sum_{k=0}^{2^{j_{0}}-1} \tilde{a}_{j_{0}, k}^{2}+2 \sum_{k=0}^{2^{j_{0}}-1} E\left(\tilde{b}_{j_{0}, k}^{2}\right)
$$

Também temos

$$
\begin{aligned}
E\left(\left(\hat{d}_{j, k}-d_{j, k}\right)^{2}\right) & =E\left(\left(\hat{d}_{j, k}-d_{j, k}^{\prime}+a_{j, k}+b_{j, k}\right)^{2}\right) \\
& \leq E\left(2\left(\hat{d}_{j, k}-d_{j, k}^{\prime}\right)^{2}+2\left(a_{j, k}+b_{j, k}\right)^{2}\right) \\
& =E\left(E_{1}\left(2\left(\hat{d}_{j, k}-d_{j, k}^{\prime}\right)^{2}\right)+2\left(a_{j, k}+b_{j, k}\right)^{2}\right)
\end{aligned}
$$

Seja $n^{-1} \sigma_{j, k ; 1}^{2}=E_{1}\left(\hat{r}_{j, k}^{2}\right)$. Denote $\min (x, y)$ por $x \wedge y$. Usando o Lema 4 de Cai e Brown (1999), obtemos

$$
E_{1}\left(\left(\hat{d}_{j, k}-d_{j, k}^{\prime}\right)^{2}\right) \leq\left(2\left(d_{j, k}^{\prime}\right)^{2}+n^{-2} \sigma_{j, k ; 1}^{2}\right) \wedge(2 \log n+1) n^{-1} \sigma_{j, k ; 1}^{2}
$$




$$
\begin{aligned}
& \leq\left(2\left(d_{j, k}^{\prime}\right)^{2}+n^{-2} \sigma_{j, k ; 1}^{2}\right) \wedge(2 \log n+\log n+1 / n) n^{-1} \sigma_{j, k ; 1}^{2} \\
& \leq\left(2\left(d_{j, k}^{\prime}\right)^{2}+n^{-2} \sigma_{j, k ; 1}^{2}\right) \wedge\left(3 n^{-1} \sigma_{j, k ; 1}^{2} \log n+n^{-2} \sigma_{j, k ; 1}^{2}\right) \\
& =2\left(d_{j, k}^{\prime}\right)^{2} \wedge 3 n^{-1} \sigma_{j, k ; 1}^{2} \log n+n^{-2} \sigma_{j, k ; 1}^{2}
\end{aligned}
$$

Agora, use este resultado em (4.17):

$$
\begin{aligned}
& E_{1}\left(2\left(\hat{d}_{j, k}-d_{j, k}^{\prime}\right)^{2}\right)+2\left(a_{j, k}+b_{j, k}\right)^{2} \\
& \leq E_{1}\left(2\left(\hat{d}_{j, k}-d_{j, k}^{\prime}\right)^{2}\right)+4 a_{j, k}^{2}+4 b_{j, k}^{2} \\
& \leq 2\left(2\left(d_{j, k}^{\prime}\right)^{2} \wedge 3 n^{-1} \sigma_{j, k ; 1}^{2} \log n+n^{-2} \sigma_{j, k ; 1}^{2}\right)+4 a_{j, k}^{2}+4 b_{j, k}^{2} \\
& =2\left(2\left(d_{j, k}+a_{j, k}+b_{j, k}\right)^{2} \wedge 3 n^{-1} \sigma_{j, k ; 1}^{2} \log n+n^{-2} \sigma_{j, k ; 1}^{2}\right)+4 a_{j, k}^{2}+4 b_{j, k}^{2} \\
& \leq 2\left(2\left(2 d_{j, k}^{2}+4 a_{j, k}^{2}+4 b_{j, k}^{2}\right) \wedge 3 n^{-1} \sigma_{j, k ; 1}^{2} \log n+n^{-2} \sigma_{j, k ; 1}^{2}\right)+4 a_{j, k}^{2}+4 b_{j, k}^{2} \\
& =2\left(\left(4 d_{j, k}^{2}+8 a_{j, k}^{2}+8 b_{j, k}^{2}\right) \wedge 3 n^{-1} \sigma_{j, k ; 1}^{2} \log n+n^{-2} \sigma_{j, k ; 1}^{2}\right)+4 a_{j, k}^{2}+4 b_{j, k}^{2} \\
& \leq 2\left(4 d_{j, k}^{2} \wedge 3 n^{-1} \sigma_{j, k ; 1}^{2} \log n+8 a_{j, k}^{2}+8 b_{j, k}^{2}+n^{-2} \sigma_{j, k ; 1}^{2}\right)+4 a_{j, k}^{2}+4 b_{j, k}^{2} \\
& =8 d_{j, k}^{2} \wedge 6 n^{-1} \sigma_{j, k ; 1}^{2} \log n+20 a_{j, k}^{2}+20 b_{j, k}^{2}+2 n^{-2} \sigma_{j, k ; 1}^{2},
\end{aligned}
$$

e portanto,

$$
\begin{aligned}
& E\left(\left(\hat{d}_{j, k}-d_{j, k}\right)^{2}\right) \\
& \quad \leq 8 d_{j, k}^{2} \wedge 6 n^{-1} E\left(\sigma_{j, k ; 1}^{2}\right) \log n+20 a_{j, k}^{2}+20 E\left(b_{j, k}^{2}\right)+2 n^{-2} E\left(\sigma_{j, k ; 1}^{2}\right) \\
& \quad=8 d_{j, k}^{2} \wedge 6 n^{-1} \sigma_{j, k}^{2} \log n+20 a_{j, k}^{2}+20 E\left(b_{j, k}^{2}\right)+2 n^{-2} \sigma_{j, k}^{2} \\
& \quad \leq 8 d_{j, k}^{2} \wedge 6 n^{-1} 2^{j} C_{2} \log n+20 a_{j, k}^{2}+20 E\left(b_{j, k}^{2}\right)+2 n^{-2} 2^{j} C_{2} .
\end{aligned}
$$

Note que,

$$
\begin{aligned}
& 8 d_{j, k}^{2} \wedge 6 n^{-1} 2^{j} C_{2} \log n=8 d_{j, k}^{2} \\
& \Leftrightarrow \quad 8 d_{j, k}^{2} \leq 6 n^{-1} 2^{j} C_{2} \log n \\
& \Leftrightarrow \quad d_{j, k}^{2} / 2^{j} \leq 6 / 8 C_{2} n^{-1} \log n .
\end{aligned}
$$

Como pelo Teorema 2.9.1 de Daubechies (1992) (veja também o Lema 1 de Cai e Brown (1999)),

$$
\frac{d_{j, k}^{2}}{2^{j}} \leq \frac{C_{4} 2^{-j(1+2 \alpha)}}{2^{j}}=C_{4} 2^{-j(2+2 \alpha)}
$$

para todo $j \geq 0$, então se existe tal $J_{1}$ que $C_{4} 2^{-j(2+2 \alpha)} \leq 6 / 8 C_{2} n^{-1} \log n$ para todo $j \geq J_{1}$, então $d_{j, k}^{2} / 2^{j} \leq C_{4} 2^{-j(2+2 \alpha)} \leq 6 / 8 C_{2} n^{-1} \log n$ e (4.18) será verdadeiro. Para encontrar $J_{1}$, note que

$$
C_{4} 2^{-j(2+2 \alpha)} \leq 6 / 8 C_{2} n^{-1} \log n
$$




$$
\begin{aligned}
& \Leftrightarrow \quad 2^{-j} \leq\left(6 C_{2} /\left(8 C_{4}\right) n^{-1} \log n\right)^{1 /(2+2 \alpha)}=C_{6}\left(\frac{\log n}{n}\right)^{1 /(2+2 \alpha)} \\
& \Leftrightarrow \quad 2^{j} \geq 1 / C_{6}\left(\frac{n}{\log n}\right)^{1 /(2+2 \alpha)} .
\end{aligned}
$$

Assim, seja $J_{1}$ o menor inteiro tal que

$$
2^{J_{1}} \geq 1 / C_{6}\left(\frac{n}{\log n}\right)^{1 /(2+2 \alpha)} .
$$

Então, como $J_{1} \leq J^{\prime}$ para $n$ suficientemente grande e $K$ apropriadamente escolhido,

$$
\begin{aligned}
& \sum_{j=j_{0}}^{J^{\prime}-1} \sum_{k=0}^{2^{j}-1} E\left(\left(\hat{d}_{j, k}-d_{j, k}\right)^{2}\right) \\
& =\frac{6 C_{2} \log n}{n} \sum_{j=j_{0}}^{J_{1}-1} \sum_{k=0}^{2^{j}-1} 2^{j}+8 \sum_{j=J_{1}}^{J^{\prime}-1} \sum_{k=0}^{2^{j}-1} d_{j, k}^{2}+20 \sum_{j=j_{0}}^{J^{\prime}-1} \sum_{k=0}^{2^{j}-1} a_{j, k}^{2}+E\left(b_{j, k}^{2}\right) \\
& +\frac{2 C_{2}}{n^{2}} \sum_{j=j_{0}}^{J^{\prime}-1} \sum_{k=0}^{2^{j}-1} 2^{j} \\
& \leq \frac{6 C_{2} \log n}{n} \sum_{j=j_{0}}^{J_{1}-1} 2^{2 j}+8 C_{4} \sum_{j=J_{1}}^{J^{\prime}-1} 2^{j} 2^{-j(1+2 \alpha)}+20 \sum_{j=j_{0}}^{J^{\prime}-1} \sum_{k=0}^{2^{j}-1} a_{j, k}^{2}+E\left(b_{j, k}^{2}\right) \\
& +\frac{2 C_{2}}{n^{2}} \sum_{j=j_{0}}^{J^{\prime}-1} 2^{2 j} \\
& \leq \frac{6 C_{2} \log n}{n}\left(\frac{2^{2 J_{1}}-2^{2 j_{0}}}{2^{2}-1}\right)+8 C_{4}\left(\frac{2^{-2 \alpha J^{\prime}}-2^{-2 \alpha J_{1}}}{2^{-2 \alpha}-1}\right) \\
& +20 \sum_{j=j_{0}}^{J^{\prime}-1} \sum_{k=0}^{2^{j}-1} a_{j, k}^{2}+E\left(b_{j, k}^{2}\right)+\frac{2 C_{2}}{n^{2}}\left(\frac{2^{2 J^{\prime}}-2^{2 j_{0}}}{2^{2}-1}\right) \\
& \leq \frac{6 C_{2} \log n}{n} 2^{2 J_{1}}+8 C_{4}\left(\frac{2^{-2 \alpha J^{\prime}}-2^{-2 \alpha J_{1}}}{2^{-2 \alpha}-1}\right)+20 \sum_{j=j_{0}}^{J^{\prime}-1} \sum_{k=0}^{2^{j}-1} a_{j, k}^{2}+E\left(b_{j, k}^{2}\right) \\
& +\frac{2 C_{2}}{n^{2}} 2^{2 J^{\prime}} \\
& =\frac{6 C_{2} \log n}{n} 2^{2 J_{1}}+2^{2 \alpha} 8 C_{4}\left(\frac{2^{-2 \alpha J^{\prime}}-2^{-2 \alpha J_{1}}}{1-2^{2 \alpha}}\right)+20 \sum_{j=j_{0}}^{J^{\prime}-1} \sum_{k=0}^{2^{j}-1} a_{j, k}^{2}+E\left(b_{j, k}^{2}\right) \\
& +\frac{2 C_{2}}{n^{2}} 2^{2 J^{\prime}} \\
& =\frac{6 C_{2} \log n}{n} 2^{2 J_{1}}+C_{7} 2^{-2 \alpha J_{1}}-C_{7} 2^{-2 \alpha J^{\prime}}+20 \sum_{j=j_{0}}^{J^{\prime}-1} \sum_{k=0}^{2^{j}-1} a_{j, k}^{2}+E\left(b_{j, k}^{2}\right)+\frac{2 C_{2}}{n^{2}} 2^{2 J^{\prime}} \text {. }
\end{aligned}
$$


Na última expressão,

$$
\begin{gathered}
\frac{6 C_{2} \log n}{n} 2^{2 J_{1}}=\frac{6 C_{2} \log n}{n} 2^{2} 2^{2\left(J_{1}-1\right)}=\frac{24 C_{2} \log n}{n}\left(2^{J_{1}-1}\right)^{2} \\
\leq \frac{24 C_{2} \log n}{n} \frac{1}{C_{6}^{2}}\left(\frac{n}{\log n}\right)^{\frac{2}{2+2 \alpha}}=C_{8}\left(\frac{\log n}{n}\right)^{\frac{2 \alpha}{2+2 \alpha}}, \\
C_{7} 2^{-2 \alpha J_{1}} \leq C_{7}\left[\frac{1}{C_{6}}\left(\frac{n}{\log n}\right)^{\frac{1}{2+2 \alpha}}\right]^{-2 \alpha}=C_{9}\left(\frac{\log n}{n}\right)^{\frac{2 \alpha}{2+2 \alpha}},
\end{gathered}
$$

e

$$
\frac{2 C_{2}}{n^{2}} 2^{2 J^{\prime}}=\frac{2 C_{2}}{n^{2}} \frac{K^{2} n}{\log n} \leq \frac{2 C_{2} K^{2}}{n} \leq \frac{2 C_{2} K^{2}}{n^{\frac{2 \alpha}{2+2 \alpha}}} \leq 2 C_{2} K^{2}\left(\frac{\log n}{n}\right)^{\frac{2 \alpha}{2+2 \alpha}} .
$$

Logo,

$$
\begin{aligned}
& \sum_{j=j_{0}}^{J^{\prime}-1} \sum_{k=0}^{2^{j}-1} E\left(\left(\hat{d}_{j, k}-d_{j, k}\right)^{2}\right) \\
& \quad \leq C_{8}\left(\frac{\log n}{n}\right)^{\frac{2 \alpha}{2+2 \alpha}}+C_{9}\left(\frac{\log n}{n}\right)^{\frac{2 \alpha}{2+2 \alpha}}+20 \sum_{j=j_{0}} \sum_{k=0}^{J^{\prime}-1} a_{j, k}^{2}+E\left(b_{j, k}^{2}\right)+2 C_{2} K^{2}\left(\frac{\log n}{n}\right)^{\frac{2 \alpha}{2+2 \alpha}} \\
& \quad=C_{10}\left(\frac{\log n}{n}\right)^{\frac{2 \alpha}{2+2 \alpha}}+20 \sum_{j=j_{0}} \sum_{k=0}^{J^{\prime}-1} a_{j, k}^{2}+E\left(b_{j, k}^{2}\right) .
\end{aligned}
$$

Agora, coletando os segundos termos dos lados direitos das desigualdades (4.16) e (4.20),

$$
2 \sum_{k=0}^{2^{j_{0}}-1} \tilde{a}_{j_{0}, k}^{2}+20 \sum_{j=j_{0}}^{J^{\prime}-1} \sum_{k=0}^{2^{j}-1} a_{j, k}^{2} \leq 20 \sum_{k=0}^{2^{j_{0}}-1} \tilde{a}_{j_{0}, k}^{2}+20 \sum_{j=j_{0}}^{\infty} \sum_{k=0}^{2^{j}-1} a_{j, k}^{2}=20\|A\|_{2}^{2}
$$

onde

$$
\begin{aligned}
\|A\|_{2}^{2} & =\int_{0}^{1} A(x)^{2} d x=\int_{0}^{1}\left[f_{n}(x)-f(x)\right]^{2} d x \\
& =\int_{0}^{1}\left[\sum_{i=0}^{n-1} n^{-1 / 2} f\left(\frac{i+1}{n+1}\right) \phi_{J, i}(x)-\sum_{i=0}^{n-1} c_{J, i} \phi_{J, i}(x)-\sum_{j=J}^{\infty} \sum_{k=0}^{2^{j}-1} d_{j, k} \psi_{j, k}(x)\right]^{2} d x \\
& =\int_{0}^{1}\left[\sum_{i=0}^{n-1}\left(n^{-1 / 2} f\left(\frac{i+1}{n+1}\right)-c_{J, i}\right) \phi_{J, i}(x)-\sum_{j=J}^{\infty} \sum_{k=0}^{2^{j}-1} d_{j, k} \psi_{j, k}(x)\right]^{2} d x
\end{aligned}
$$




$$
\begin{aligned}
& \leq \int_{0}^{1} 2\left[\sum_{i=0}^{n-1}\left(n^{-1 / 2} f\left(\frac{i+1}{n+1}\right)-c_{J, i}\right) \phi_{J, i}(x)\right]^{2} d x+\int_{0}^{1} 2\left[\sum_{j=J}^{\infty} \sum_{k=0}^{2^{j}-1} d_{j, k} \psi_{j, k}(x)\right]^{2} d x \\
& \leq 2 \sum_{i=0}^{n-1}\left(n^{-1 / 2} f\left(\frac{i+1}{n+1}\right)-c_{J, i}\right)^{2}+2 \sum_{j=J}^{\infty} \sum_{k=0}^{2^{j}-1} d_{j, k}^{2} \\
& =2 \sum_{i=0}^{n-1}\left(n^{-1 / 2} f\left(\frac{i+1}{n+1}\right)-c_{J, i}\right)^{2}+O\left(n^{-2 \alpha}\right) \leq C_{11} n^{-2(1 / 2+s(\alpha))},
\end{aligned}
$$

pela Proposição 1 e equação (11) em Cai e Brown (1999).

Similarmente, coletando os terceiros termos dos lados direitos das desigualdades (4.16) e (4.20),

$$
\begin{aligned}
& \sum_{k=0}^{2^{j_{0}}-1} E\left(\tilde{b}_{j_{0}, k}^{2}\right)+\sum_{j=j_{0}}^{J^{\prime}-1} \sum_{k=0}^{2^{j}-1} E\left(b_{j, k}^{2}\right) \\
& \quad \leq E\|B\|_{2}^{2}=E\left(E_{1}\|B\|_{2}^{2}\right) \\
& \quad \leq E\left(E_{1}\left(\sum_{i=0}^{n-1}\left[n^{-1 / 2} f\left(t_{(i+1)}\right)-n^{-1 / 2} f(i /(n+1))\right]^{2}\right)\right) \\
& \quad E\left(E_{1}\left(1 / n \sum_{i=1}^{n}\left[f\left(t_{(i)}\right)-f(i /(n+1))\right]^{2}\right)\right) \\
& \leq E\left(C_{12} n^{-s(\alpha)}\right)=C_{12} n^{-s(\alpha)},
\end{aligned}
$$

usando o Lema 3 de Cai e Brown (1999).

Finalmente, de (4.15) e dos resultados que o seguem, temos

$$
\begin{aligned}
E\left(\|\hat{f}-f\|_{2}^{2}\right) \leq & C_{3} 2^{2 j_{0}} / n+C_{13} / n^{2(1 / 2+s(\alpha))}+C_{14} / n^{s(\alpha)}+C_{10}(\log n / n)^{2 \alpha /(2+2 \alpha)} \\
& +C_{5} K^{2 \alpha}(\log n / n)^{2 \alpha /(2+2 \alpha)}
\end{aligned}
$$

Mas

$$
C_{3} 2^{2 j_{0}} / n \leq C_{3} 2^{2 j_{0}} / n^{2 \alpha /(2+2 \alpha)} \leq C_{15}(\log n / n)^{2 \alpha /(2+2 \alpha)},
$$

e para todo $\alpha>0, s(\alpha)=\min \{\alpha, 1\} \geq 2 \alpha /(2+2 \alpha)$ e

$$
C_{14} / n^{s(\alpha)} \leq C_{14}(\log n / n)^{2 \alpha /(2+2 \alpha)} .
$$

Também, como $s(\alpha) \geq 0$,

$$
C_{13} / n^{2(1 / 2+s(\alpha))} \leq C_{13} / n \leq C_{13}(\log n / n)^{2 \alpha /(2+2 \alpha)} .
$$


Portanto,

$$
E\left(\|\hat{f}-f\|_{2}^{2}\right) \leq C_{16}(\log n / n)^{2 \alpha /(2+2 \alpha)}
$$

para $\alpha \geq 0$ e $n$ suficientemente grande.

\subsubsection{Prova do Corolário 1}

Como $T=1$ e $t_{i}=(i-1 / 2) / n+j_{i}$, onde $j_{i}$ são i.i.d. Uniforme $[-1 /(2 n), 1 /(2 n)]$, então $E\left(t_{i}\right)=$ $(i-1 / 2) / n$ e $\operatorname{Var}\left(t_{i}\right)=\operatorname{Var}\left(j_{i}\right)=1 /\left(12 n^{2}\right)$, para todo $i=1,2, \ldots, n$. Isto afeta apenas o Lema 3 de Cai e Brown (1999) mas, para uma função $f \in \Lambda^{\alpha}(M)$ fixa, então $|f(x)-f(y)| \leq C_{17}|x-y|^{s(\alpha)}$, onde $s(\alpha)=\min (\alpha, 1)$. Logo,

$$
\frac{1}{n} \sum_{i=1}^{n} E\left(f\left(t_{i}\right)-f(i / n)\right)^{2} \leq \frac{C_{18}}{n} \sum_{i=1}^{n}\left(\operatorname{Var}\left(t_{i}\right)+\frac{1}{2 n^{2}}\right)^{s(\alpha)} \leq C_{18} n^{-s(\alpha)},
$$

e o Lema 3 de Cai e Brown (1999) continua válido. Consequentemente, a prova do Teorema 3 continua válida também. 


\section{Capítulo 5}

\section{Delineamento Aleatório Geral}

\subsection{Procedimento Usando Ondaletas Deformadas}

Suponha que sejam observados os pares de variáveis aleatórias $\left(Y_{1}, X_{1}\right), \ldots,\left(Y_{n}, X_{n}\right), n=2^{J}$, e que se formule o modelo

$$
Y_{t}=f\left(X_{t}\right)+\epsilon_{t},
$$

onde a função $f$ é desconhecida e $\epsilon_{t}$ é um termo de erro. Nosso objetivo é estimar a função $f$ por $\hat{f}$, com baixo risco

$$
R(f, \hat{f})=E\|f-\hat{f}\|_{p}^{p}=E\left(\int|f(x)-\hat{f}(x)|^{p} d x\right),
$$

para alguma ampla classe de funções.

Antes de se listar algumas suposições, é necessária a seguinte

Definição 6 Seja $\left\{\epsilon_{t}\right\}_{t \in \mathbb{R}}$ um processo estocástico, $\mathcal{E}_{A}$ a $\sigma$-álgebra gerada por $\left\{\epsilon_{t}: t \in A \subset \mathbb{R}\right\}$, e similarmente $\mathcal{E}_{B}$. Para algum $h>0$, tome $A$ e $B$ tais que $|a-b|>h$, se $a \in A$ e $b \in B$. Defina

1. $\alpha_{\epsilon, h}\left(\mathcal{E}_{A}, \mathcal{E}_{B}\right)=\sup \left\{|P(U) P(V)-P(U \cap V)|: U \in \mathcal{E}_{A}, V \in \mathcal{E}_{B}\right\}$ como o correspondente coeficiente mixing forte;

2. $\rho_{\epsilon, h}\left(\mathcal{E}_{A}, \mathcal{E}_{B}\right)=\sup \left\{|\operatorname{Corr}(U, V)|: U \in L_{2}\left(\mathcal{E}_{A}\right), V \in L_{2}\left(\mathcal{E}_{B}\right)\right\}$ como o correspondente coeficiente $\rho$-mixing.

Para alcançar este objetivo de risco mínimo, fazem-se as seguintes suposições:

1. a função $f$ é limitada e possui suporte compacto no intervalo $[a, b]$;

2. $\left\{X_{t}\right\}_{t \in \mathbb{Z}}$ são variáveis aleatórias i.i.d. com função distribuição $G(x)$ e respectiva densidade $g(x)$ com suporte no intervalo $[a, b]$;

3. a função distribuição $G(x)$ e sua inversa $G^{-1}(x)$ são contínuas e estritamente monótonas, i.e., $G^{-1}(G(x))=x$ q.c. e $G\left(G^{-1}(x)\right)=x$ q.c., para todo $x \in[a, b]$; 
4. a função $w(x)=\left[g\left(G^{-1}(x)\right)\right]^{-1}$ é um peso de Muckenhoupt: $w \in A_{p}([a, b])$. Se $0<g<M<\infty$, então $w(x) \in A_{\infty}$;

5. os erros $\left\{\epsilon_{t}\right\}_{t \in \mathbb{Z}}$ são uma sequência estacionária gaussiana com média zero, variância $\sigma^{2}<\infty$, $\operatorname{Cov}\left(\epsilon_{r}, \epsilon_{s}\right)=\sigma_{r, s}=\gamma(|r-s|)$, para todo $r \neq s, r, s \in \mathbb{Z}$ e $\epsilon_{r}$ independente de $X_{s}$, para todo $r, s \in \mathbb{Z}$. Sem perda de generalidade, suponha que $\sigma^{2}=1$;

6. existem $c \geq p, c \in 2 \mathbb{N}$ e $\delta>0$ que satisfazem a condição mixing $\sum_{h=1}^{\infty}(h+1)^{c-2}\left(\alpha_{\epsilon, h}\right)^{\delta /(c+\delta)}<$ $\infty$, para o coeficiente mixing forte $\alpha_{\epsilon, h}$, como definido em Doukhan (1994), p. 18.

As suposições 1 a 4 são usuais em regressão não-paramétrica usando ondaletas deformadas quando os erros são i.i.d. (KERKYACHARIAN; PICARD, 2004). Quando os erros são correlacionados, se requer que eles sejam estacionários e satisfaçam uma específica condição mixing.

Seja $0<C<\infty$ uma constante geral que não depende do tamanho $n$ da amostra. A função $f$ será estimada por meio de métodos de ondaletas por

$$
\hat{f}(x)=\hat{c}_{0,0} \phi_{0,0}(G(x))+\sum_{j=0}^{J_{1}} \sum_{k=0}^{2^{j}-1} \hat{d}_{j, k} \eta_{H}\left(\hat{d}_{j, k}, \kappa t_{n}\right) \psi_{j, k}(G(x)),
$$

para algum $\kappa>0$, com

$$
t_{n}=\left(\frac{\log n}{n}\right)^{1 / 2}
$$

onde $2^{J_{1}} \leq C \min \left\{n_{1}, n_{2}\right\}$,

$$
\begin{gathered}
n_{1}=\left(\frac{n}{\log n}\right)^{1 / 2}, \quad n_{2}=\left(\frac{n^{(3 p-2) / p}}{\log n}\right)^{\frac{p+\delta}{p+\delta-2}}, \\
\hat{d}_{j, k}=\frac{1}{n} \sum_{t=1}^{n} \psi_{j, k}\left(G\left(X_{t}\right)\right) Y_{t}
\end{gathered}
$$

e similarmente para $\hat{c}_{0,0}$.

Note que para todo $\delta>0$, se $p \geq 2$, então $n_{1} \leq n_{2}$. Isto também é verdadeiro, se $1<p<2$ e $\delta \geq 1$. Portanto, precisa-se preocupar apenas se $1<p<2$ e $0<\delta<1$, que é o caso em que $n_{2}$ pode ser menor do que $n_{1}$.

Se a função distribuição $G$ não for conhecida, usa-se a distribuição empírica, independentemente estimada pela mesma quantidade de observações, e o novo estimador será denotado por $\hat{c}_{0,0}^{\prime}, \hat{d}_{j, k}^{\prime}$ e $\hat{f}^{\prime}(x)$. 


\subsubsection{Resultados Principais}

Sejam $c_{0,0}$ e $d_{j, k}$ os coeficientes da função $f\left(G^{-1}(y)\right)$ na base de ondaletas $\phi_{0,0} \cup\left\{\psi_{j, k}, j \geq 0, k=\right.$ $\left.0, \ldots, 2^{j}-1\right\}$ :

$$
f\left(G^{-1}(y)\right)=c_{0,0} \phi_{0,0}(x)+\sum_{j=0}^{\infty} \sum_{k=0}^{2^{j}-1} d_{j, k} \psi_{j, k}(x) .
$$

Proposição 4 Para qualquer $j \geq 0$, e qualquer $k=0,1, \ldots, 2^{j}-1$, sob as suposições 1 a 5 , o estimador (5.3) satisfaz:

1. $E\left(\hat{d}_{j, k}\right)=d_{j, k}$

2. $\operatorname{Var}\left(\hat{d}_{j, k}\right)=n^{-1}\left[\int_{0}^{1} f^{2}\left(G^{-1}(y)\right) \psi_{j, k}^{2}(y) d y-d_{j, k}^{2}+\sigma^{2}\right]$;

3. $\operatorname{Cov}\left(\hat{d}_{j, k}, \hat{d}_{j^{\prime}, k^{\prime}}\right)=n^{-1}\left[\int_{0}^{1} f^{2}\left(G^{-1}(y)\right) \psi_{j, k}(y) \psi_{j^{\prime}, k^{\prime}}(y) d y-d_{j, k} d_{j^{\prime}, k^{\prime}}\right]$, for $j \neq j^{\prime}, k \neq k^{\prime}$.

Note que a variância e a covariância do estimador não são influenciadas pela correlação dos erros. Especificamente, a covariância não é zero por causa do delineamento aleatório.

Proposição 5 Seja $0<C<\infty$ uma constante que não depende do tamanho $n$ da amostra. Sejam $n_{1}$ e $n_{2}$ dados por (5.2), $t_{n}$ dado por (5.1) e $p>1$. Então, para quaiquer $j=0, \ldots, J_{1}, 2^{J_{1}} \leq$ $C \min \left\{n_{1}, n_{2}\right\}$, e qualquer $k=0,1, \ldots, 2^{j}-1$, sob as suposiçôes 1 to 6 , o estimador (5.3) satisfaz:

1. $E\left(\left|\hat{d}_{j, k}-d_{j, k}\right|^{2 p}\right) \leq C t_{n}^{2 p}=O\left(\left(\frac{\log n}{n}\right)^{p}\right)$

2. $P\left(\left|\hat{d}_{j, k}-d_{j, k}\right| \geq \kappa t_{n} / 2\right) \leq \min \left\{C t_{n}^{2 p}, t_{n}^{4}\right\}=\min \left\{O\left(\left(\frac{\log n}{n}\right)^{p}\right), O\left(\left(\frac{\log n}{n}\right)^{2}\right)\right\}$, para algum $\kappa>0$.

A Proposição 4 é usada na demonstração da Proposição 5. Para se aplicar a Proposição 5, são necessárias as seguintes definições.

Definição 7 Considere uma sequência de modelos $\mathcal{E}_{n}=\left\{P_{\theta}^{n}, \theta \in \Theta\right\}$, onde $P_{\theta}^{n}$ são distribuições de probabilidade e $\Theta$ é o espaço paramétrico. Considere também uma sequência $\hat{q}_{n}$ de estimadores de uma quantidade $q(\theta)$, uma função perda $L\left(\hat{q}_{n}, q(\theta)\right)$ e uma taxa de convergência $a_{n}$ tendendo a zero. Então, o conjunto maximal associado com a sequência $\hat{q}_{n}$, a função perda $L$, a taxa $a_{n}$ e uma constante $T$, é o conjunto

$$
\operatorname{Max}\left(\hat{q}_{n}, L, a_{n}\right)(T)=\left\{\theta \in \Theta, \sup _{n} E_{\theta} L\left(\hat{q}_{n}, q(\theta)\right) a_{n}^{-1} \leq T\right\} .
$$


A avaliação de conjuntos maximais é um modo de se mensurar o desempeno de procedimentos estatísticos (veja, por exemplo Kerkyacharian e Picard (2000)). Possui a vantagem de fornecer comparações de procedimentos menos pessimistas do que a abordagem minimax. Porém, enquanto o risco minimax pode ser calculado em muitas situações para um determinado tamanho de amostra, a noção de conjunto maximal está associada a uma sequência de procedimentos estatísticos e à uma taxa de convergência sendo, portanto, de natureza puramente assintótica.

Definição 8 Para $p>1, j \in \mathbb{N}$ e $k \in \mathbb{Z}$, seja $\nu$ a medida

$$
\nu\{(j, k)\}=2^{j p / 2} \omega\left(I_{j, k}\right)
$$

onde $\omega$ é um peso de Muckenhoupt e $I_{j, k}$ é um intervalo qualquer da reta indexado por $j$ e $k$. Usando esta medida, defina o seguinte espaço de funçôes:

$$
\begin{aligned}
l_{q, \infty}(\nu)= & \left\{f(x)=\sum_{k \in \mathbb{Z}} c_{0, k} \phi_{0, k}(G(x))+\sum_{j \geq 0} \sum_{k \in \mathbb{Z}} d_{j, k} \psi_{j, k}(G(x)),\right. \\
& \left.\sup _{t>0} t^{q} \nu\left\{(j, k):\left|c_{0, k}\right|>t,\left|d_{j, k}\right|>t\right\}<\infty\right\} .
\end{aligned}
$$

Pelo Teorema 1 (p.12), a base de ondaletas deformadas satisfaz às propriedades de encolhimento e $p$-Temlyakov. Sob as suposições 1 a 6, o Teorema 5 de Kerkyacharian e Picard (2004) informa que

$$
\sup _{n} \nu\left\{\Lambda_{n}\right\}\left(\frac{\log n}{n}\right)^{p / 2}<\infty
$$

onde $\Lambda_{n}=\left\{(j, k):|k| \leq N 2^{j}\right\}$, para $0 \leq j \leq J$, e onde $N$ é o comprimento do suporte da ondaletamãe $\psi$. Estes dois teoremas, juntamente com o resultado da Proposição 5, fazem com que o Teorema 4 de Kerkyacharian e Picard (2004) possa ser aplicado para se obter o seguinte teorema.

Teorema 4 (KERKYACHARIAN; PICARD, 2004, Teorema 3) Seja $p>1,0<q<p$. Sob as suposiçôes 1 a 6 , o conjunto maximal associado ao estimador $\hat{f}$,

$$
\operatorname{Max}\left(\hat{f}, L_{p}, t_{n}^{p-q}\right)=\left\{f, E\|\hat{f}-f\|_{p}^{p}\left(\frac{\log n}{n}\right)^{(q-p) / 2}<\infty\right\},
$$

pode ser expresso como

$$
\operatorname{Max}\left(\hat{f}, L_{p}, t_{n}^{p-q}\right)=l_{q, \infty}(\nu) \cap\left\{f(x)=\sum_{k \in \mathbb{Z}} c_{0, k} \phi_{0, k}(G(x))+\sum_{j \geq 0} \sum_{k \in \mathbb{Z}} d_{j, k} \psi_{j, k}(G(x)),\right.
$$




$$
\left.\sup _{l>0}\left\|\sum_{k \in \mathbb{Z}} c_{0, k} \phi_{0, k}(G(x))+\sum_{j \geq l} \sum_{k \in \mathbb{Z}} d_{j, k} \psi_{j, k}(G(x))\right\|_{p}^{p} 2^{l(p-q)}<\infty\right\} .
$$

quando $\nu\{(j, k)\}=2^{j p / 2} \omega\left(I_{j, k}\right)$.

Finalmente, pela Proposição 4, Proposição 2 e Teorema 2, todos em Kerkyacharian e Picard (2004), segue que o estimador $\hat{f}$ é quase-minimax para uma grande amplitude de classes de funções.

\subsubsection{Comentários}

A abordagem de ondaletas deformadas tenta responder para quais classes de delineamentos é ótima a aplicação dos métodos usuais de ondaletas, ignorando a irregularidade do delineamento. Neste sentido, ela de certa forma é uma generalização de Cai e Brown (1999) e do Capítulo 4.

Um método concorrente é o de Cai e Brown (1998) e comparações teóricas são desejadas (KERKYACHARIAN; PICARD, 2004). Comparações numéricas foram realizadas para erros i.i.d. (CHESNEAU; WILLER, 2007) e concluiu-se que os estimadores usando ondaletas deformadas são ligeiramente melhores. A diferença é maior quando a densidade do delineamento é desconhecida e é nula em alguns pontos. Entretanto, como a proposta de ondaletas deformadas se utiliza de limiares duros apenas, o resultado visual é ligeiramente pior do que o método de Cai e Brown (1998).

A questão de comparação fica mais evidente quando se observa que a teoria de ondaletas deformadas se utiliza da noção de conjunto maximal e é conectada a uma sequência de procedimentos estatísticos juntamente com uma taxa de convergência (veja, por exemplo, Kerkyacharian e Picard (2000)). É portanto de natureza puramente assintótica. Dificuldades podem ocorrer quando dois procedimentos produzem conjuntos maximais "não aninhados" que são ordenados diferentemente, do ponto de vista de conjuntos maximais, para diferentes taxas de convergência.

Note que a condição mixing exigida equivale à de autocovariâncias absolutamente somáveis, quando $c=p=2$ e $\delta \rightarrow \infty$. Assim, há uma certa generalização dos resultados do Capítulo 4, para estruturas de erros e funções perda mais gerais.

\subsection{Provas da Seção 5.1}

\subsubsection{Prova da Proposição 4}

Item (1) é provado em Kerkyacharian e Picard (2004, p. 1059). Item (2) é um caso especial do item (3):

$$
\operatorname{Cov}\left(\hat{d}_{j, k}, \hat{d}_{j^{\prime}, k^{\prime}}\right)=\operatorname{Cov}\left(n^{-1} \sum_{r=1}^{n} \psi_{j, k}\left(G\left(X_{r}\right)\right)\left[f\left(X_{r}\right)+\epsilon_{r}\right], n^{-1} \sum_{s=1}^{n} \psi_{j^{\prime}, k^{\prime}}\left(G\left(X_{s}\right)\right)\left[f\left(X_{s}\right)+\epsilon_{s}\right]\right)
$$




$$
\begin{gathered}
=n^{-2} \sum_{r=1}^{n} \sum_{s=1}^{n} \operatorname{Cov}\left(\psi_{j, k}\left(G\left(X_{r}\right)\right)\left[f\left(X_{r}\right)+\epsilon_{r}\right], \psi_{j^{\prime}, k^{\prime}}\left(G\left(X_{s}\right)\right)\left[f\left(X_{s}\right)+\epsilon_{s}\right]\right) \\
=n^{-2} \sum_{r=1}^{n} \sum_{s=1}^{n}\left[\operatorname{Cov}\left(\psi_{j, k}\left(G\left(X_{r}\right)\right) f\left(X_{r}\right), \psi_{j^{\prime}, k^{\prime}}\left(G\left(X_{s}\right)\right) f\left(X_{s}\right)\right)\right. \\
+\operatorname{Cov}\left(\psi_{j, k}\left(G\left(X_{r}\right)\right) f\left(X_{r}\right), \psi_{j^{\prime}, k^{\prime}}\left(G\left(X_{s}\right)\right) \epsilon_{s}\right) \\
\left.+\operatorname{Cov}\left(\psi_{j, k}\left(G\left(X_{r}\right)\right) \epsilon_{r}, \psi_{j^{\prime}, k^{\prime}}\left(G\left(X_{s}\right)\right) f\left(X_{s}\right)\right)+\operatorname{Cov}\left(\psi_{j, k}\left(G\left(X_{r}\right)\right) \epsilon_{r}, \psi_{j^{\prime}, k^{\prime}}\left(G\left(X_{s}\right)\right) \epsilon_{s}\right)\right] \\
=n^{-2} \sum_{r=1}^{n} \sum_{s=1}^{n}[A+B+C+D] .
\end{gathered}
$$

Agora,

$$
A=E\left(\psi_{j, k}\left(G\left(X_{r}\right)\right) f\left(X_{r}\right) \psi_{j^{\prime}, k^{\prime}}\left(G\left(X_{s}\right)\right) f\left(X_{s}\right)\right)-E\left(\psi_{j, k}\left(G\left(X_{r}\right)\right) f\left(X_{r}\right)\right) E\left(\psi_{j^{\prime}, k^{\prime}}\left(G\left(X_{s}\right)\right) f\left(X_{s}\right)\right)
$$

tal que, se $r=s$,

$$
A=E\left(\psi_{j, k}(G(X)) \psi_{j^{\prime}, k^{\prime}}(G(X)) f^{2}(X)\right)-E\left(\psi_{j, k}(G(X)) f(X)\right) E\left(\psi_{j^{\prime}, k^{\prime}}(G(X)) f(X)\right)
$$

$\mathrm{e}$, se $r \neq s$,

$$
A=E\left(\psi_{j, k}(G(X)) f(X)\right) E\left(\psi_{j^{\prime}, k^{\prime}}(G(X)) f(X)\right)-E\left(\psi_{j, k}(G(X)) f(X)\right) E\left(\psi_{j^{\prime}, k^{\prime}}(G(X)) f(X)\right) .
$$

Portanto, se $r=s$,

$$
A=\int_{a}^{b} f^{2}(x) \psi_{j, k}(G(x)) \psi_{j^{\prime}, k^{\prime}}(G(x)) d x-d_{j, k} d_{j^{\prime}, k^{\prime}}=\int_{0}^{1} f^{2}\left(G^{-1}(y)\right) \psi_{j, k}(y) \psi_{j^{\prime}, k^{\prime}}(y) d x-d_{j, k} d_{j^{\prime}, k^{\prime}}
$$

e $A=0$ se $r \neq s$.

Agora,

$$
\begin{gathered}
B=E\left(\psi_{j, k}\left(G\left(X_{r}\right)\right) f\left(X_{r}\right) \psi_{j^{\prime}, k^{\prime}}\left(G\left(X_{s}\right)\right) \epsilon_{s}\right)-E\left(\psi_{j, k}\left(G\left(X_{r}\right)\right) f\left(X_{r}\right)\right) E\left(\psi_{j^{\prime}, k^{\prime}}\left(G\left(X_{s}\right)\right) \epsilon_{s}\right) \\
=E\left(\psi_{j, k}\left(G\left(X_{r}\right)\right) f\left(X_{r}\right) \psi_{j^{\prime}, k^{\prime}}\left(G\left(X_{s}\right)\right)\right) E\left(\epsilon_{s}\right)-E\left(\psi_{j, k}\left(G\left(X_{r}\right)\right) f\left(X_{r}\right)\right) E\left(\psi_{j^{\prime}, k^{\prime}}\left(G\left(X_{s}\right)\right)\right) E\left(\epsilon_{s}\right) .
\end{gathered}
$$

Portanto, $B=0$ e, por simetria, $C=0$.

Finalmente,

$$
\begin{gathered}
\left.\left.D=E\left(\psi_{j, k}\left(G\left(X_{r}\right)\right) \epsilon_{r}\right) \psi_{j^{\prime}, k^{\prime}}\left(G\left(X_{s}\right)\right) \epsilon_{s}\right)-E\left(\psi_{j, k}\left(G\left(X_{r}\right)\right) \epsilon_{r}\right)\right) E\left(\psi_{j^{\prime}, k^{\prime}}\left(G\left(X_{s}\right)\right) \epsilon_{s}\right) \\
\left.=E\left(\psi_{j, k}\left(G\left(X_{r}\right)\right)\right) \psi_{j^{\prime}, k^{\prime}}\left(G\left(X_{s}\right)\right)\right) E\left(\epsilon_{r} \epsilon_{s}\right) .
\end{gathered}
$$


Portanto, se $r=s$

$$
D=\sigma^{2} E\left(\psi_{j, k}(G(X)) \psi_{j^{\prime}, k^{\prime}}(G(X))\right)=\sigma^{2} \int_{0}^{1} \psi_{j, k}(y) \psi_{j^{\prime}, k^{\prime}}(y)
$$

e

$$
\left.D=\sigma_{r, s} E\left(\psi_{j, k}(G(X))\right) \psi_{j^{\prime}, k^{\prime}}(G(X))\right)=0 .
$$

se $r \neq s$.

Como os únicos termos não nulos ocorrem quando $r=s$, então

$$
n^{-2} \sum_{r=1}^{n} \sum_{s=1}^{n}[A+B+C+D]=n^{-1}[A+B+C+D] .
$$

\subsubsection{Prova da Proposição 5, Item 1}

$$
\begin{aligned}
E\left(\left|\hat{d}_{j, k}-d_{j, k}\right|^{p}\right) & =E\left(\left|n^{-1} \sum_{t=1}^{n} \psi_{j, k}\left(G\left(X_{t}\right)\right) Y_{t}-d_{j, k}\right|^{p}\right) \\
& =E\left(n^{-p}\left|\sum_{t=1}^{n}\left[\psi_{j, k}\left(G\left(X_{t}\right)\right) Y_{t}-d_{j, k}\right]\right|^{p}\right) \\
& =E\left(n^{-p}\left|\sum_{t=1}^{n} Z_{t ; j, k}\right|^{p}\right) .
\end{aligned}
$$

Seja $0<C<\infty$ uma constante geral que não depende do tamanho $n$ da amostra. Pela Proposição $4, E\left(Z_{t ; j, k}\right)=0$ para todo $t, j, k$ considerado. Também, para todo $r \neq s$,

$$
\begin{aligned}
\operatorname{Cov}\left(Z_{r ; j, k}, Z_{s ; j, k}\right)= & \operatorname{Cov}\left(\psi_{j, k}\left(G\left(X_{r}\right)\right) Y_{r}, \psi_{j, k}\left(G\left(X_{s}\right)\right) Y_{s}\right) \\
= & \operatorname{Cov}\left(\psi_{j, k}\left(G\left(X_{r}\right)\right) f\left(X_{r}\right), \psi_{j, k}\left(G\left(X_{s}\right)\right) f\left(X_{s}\right)\right) \\
& +\operatorname{Cov}\left(\psi_{j, k}\left(G\left(X_{r}\right)\right) f\left(X_{r}\right), \psi_{j, k}\left(G\left(X_{s}\right)\right) \epsilon_{s}\right) \\
& +\operatorname{Cov}\left(\psi_{j, k}\left(G\left(X_{r}\right)\right) \epsilon_{r}, \psi_{j, k}\left(G\left(X_{s}\right)\right) f\left(X_{s}\right)\right) \\
& +\operatorname{Cov}\left(\psi_{j, k}\left(G\left(X_{r}\right)\right) \epsilon_{r}, \psi_{j, k}\left(G\left(X_{s}\right)\right) \epsilon_{s}\right) \\
= & 0+0+0+E\left(\psi_{j, k}\left(G\left(X_{r}\right)\right) \epsilon_{r} \psi_{j, k}\left(G\left(X_{s}\right)\right) \epsilon_{s}\right) \\
= & E\left(\psi_{j, k}(G(X))\right) E\left(\epsilon_{r} \epsilon_{s}\right)=0 .
\end{aligned}
$$

Pela suposição 2, o coeficiente mixing $\alpha_{\mathbf{X}, h}=0$, como dado em Doukhan (1994), p.18. Similarmente, defina o coeficiente mixing $\alpha_{\mathbf{Z}_{j, k}, h}$. Como este coeficiente mixing é definido nas $\sigma$-álgebras, então ele apenas pode ser menor ou igual a $\alpha_{\epsilon, h}$ e, pela Suposição $6, \sum_{h=1}^{\infty}(h+1)^{c-2}\left(\alpha_{\mathbf{Z}_{j, k}, h}\right)^{\delta /(c+\delta)}<\infty$. 
Portanto, pelo Teorema 2 em Doukhan (1994), para $p>2$,

$$
n^{-p} E\left(\left|\sum_{t=1}^{n} Z_{t ; j, k}\right|^{p}\right) \leq C / n^{p} \max \left\{\sum_{t=1}^{n}\left[E\left(\left|Z_{t ; j, k}\right|^{p+\delta}\right)\right]^{\frac{p}{p+\delta}},\left[\sum_{t=1}^{n}\left(E\left(\left|Z_{t ; j, k}\right|^{2+\delta}\right)\right)^{\frac{2}{2+\delta}}\right]^{p / 2}\right\} .
$$

Usando o Lema 1 a seguir, isso é menor ou igual a

$$
\begin{gathered}
C / n^{p}\left\{\sum_{t=1}^{n}\left[E\left(\left|\psi_{j, k}\left(G\left(X_{t}\right)\right) Y_{t}\right|^{p+\delta}\right)\right]^{\frac{p}{p+\delta}}+\left[\sum_{t=1}^{n}\left(E\left(\left|\psi_{j, k}\left(G\left(X_{t}\right)\right) Y_{t}\right|^{2+\delta}\right)\right)^{\frac{2}{2+\delta}}\right]^{p / 2}\right\} \\
=C\left\{\frac{\left[E\left(\left|\psi_{j, k}\left(G\left(X_{t}\right)\right) Y_{t}\right|^{p+\delta}\right)\right]^{\frac{p}{p+\delta}}}{n^{p-1}}+\frac{\left(E\left(\left|\psi_{j, k}\left(G\left(X_{t}\right)\right) Y_{t}\right|^{2+\delta}\right)\right)^{\frac{p}{2+\delta}}}{n^{p / 2}}\right\} .
\end{gathered}
$$

Pelo resultado da linha 19 de Kerkyacharian e Picard (2004, p. 1086), esta última expressão é menor ou igual a

$$
C\left\{\frac{\left(1+\|f\|_{\infty}^{p+\delta}\right)^{\frac{p}{p+\delta}} 2^{j\left(\frac{p+\delta}{2}-1\right) \frac{p}{p+\delta}}}{n^{p-1}}+\frac{\left(1+\|f\|_{\infty}^{2+\delta}\right)^{\frac{2}{p+\delta}} 2^{j\left(\frac{p+\delta}{2}-1\right) \frac{p}{p+\delta}}}{n^{p / 2}}\right\}=C(A+B) .
$$

Para $p>2$,

$$
\begin{aligned}
A \leq C(n / \log n)^{p / 2} & \Leftrightarrow 2^{j((p+\delta) / 2-1) p /(p+\delta)} \leq C n^{3 p / 2-1} /(\log n)^{p / 2} \\
& \Leftrightarrow 2^{j(p+\delta-2) p /(2(p+\delta))} \leq C n^{(3 p-2) / 2} /(\log n)^{p / 2} \\
& \Leftrightarrow 2^{j} \leq C\left(\frac{n^{(3 p-2) / p}}{\log n}\right)^{\frac{p+\delta}{p+\delta-2}} .
\end{aligned}
$$

Similarmente,

$$
\begin{aligned}
B \leq C(n / \log n)^{p / 2} & \Leftrightarrow 2^{j((p+\delta) / 2-1) p /(p+\delta)} \leq C n^{p} /(\log n)^{p / 2} \\
& \Leftrightarrow 2^{j(p+\delta-2) p /(2(p+\delta))} \leq C n^{p} /(\log n)^{p / 2} \\
& \Leftrightarrow 2^{j} \leq C\left(\frac{n^{2}}{\log n}\right)^{\frac{p+\delta}{p+\delta-2}} \leq C\left(\frac{n^{(3 p-2) / p}}{\log n}\right)^{\frac{p+\delta}{p+\delta-2}} .
\end{aligned}
$$

Para $1<p \leq 2$, novamente por Suposição 6 e Teorema 2 em Doukhan (1994),

$$
n^{-p} E\left(\left|\sum_{t=1}^{n} Z_{t ; j, k}\right|^{p}\right) \leq C / n^{p} \sum_{t=1}^{n}\left[E\left(\left|Z_{t ; j, k}\right|^{p+\delta}\right)\right]^{\frac{p}{p+\delta}}
$$

e, usando o seguinte Lema 1 e o resultado na linha 19 de Kerkyacharian e Picard (2004, p.1086), isto 
é menor ou igual a

$$
\begin{aligned}
& C / n^{p} \sum_{t=1}^{n}\left[E\left(\left|\psi_{j, k}\left(G\left(X_{t}\right)\right) Y_{t}\right|^{p+\delta}\right)\right]^{\frac{p}{p+\delta}} \\
& \leq C / n^{p-1}\left[\left(1+\|f\|_{\infty}^{p+\delta}\right) 2^{j\left(\frac{p+\delta}{2}-1\right)}\right]^{\frac{p}{p+\delta}} .
\end{aligned}
$$

Como no último caso, isto será limitado superiormente por $C(n / \log n)^{p / 2}$ se

$$
2^{j} \leq C\left(\frac{n^{(3 p-2) / p}}{\log n}\right)^{\frac{p+\delta}{p+\delta-2}} .
$$

Note que

$$
\left(\frac{n}{\log n}\right)^{1 / 2} \leq\left(\frac{n^{(3 p-2) / p}}{\log n}\right)^{\frac{p+\delta}{p+\delta-2}}
$$

para todo $\delta>0$, quando $p \geq 2$ e, para $1<p<2$, também é verdadeiro se $\delta \geq 1$. Isto pode não ser verdadeiro apenas se $1<p<2$ and $0<\delta<1$. Então,

$$
E\left(\left|\hat{d}_{j, k}-d_{j, k}\right|^{p}\right) \leq C(n / \log n)^{p / 2}
$$

para todo $2^{J_{1}} \leq C \min \left\{n_{1}, n_{2}\right\}$, onde

$$
n_{1}=\left(\frac{n}{\log n}\right)^{1 / 2}, \quad n_{2}=\left(\frac{n^{(3 p-2) / p}}{\log n}\right)^{\frac{p+\delta}{p+\delta-2}}
$$

Lema 1 Seja $X$ uma variável aleatória com média finita $\mu$ e momento finito de ordem $p$. Então $E\left(|X-\mu|^{p}\right) \leq C E\left(|X|^{p}\right)$, para alguma constante $C$ que depende de $p$.

Prova. Pela desigualdade de Hölder, $E\left(|X-\mu|^{p}\right)=\int_{0}^{\infty} P\left(|X-\mu|^{p}>y\right) d y$ é menor ou igual a

$$
\begin{aligned}
\int_{0}^{\infty} P\left(2^{p-1}\left(|X|^{p}+|-\mu|^{p}\right)>y\right) d y & =\int_{0}^{\infty} P\left(|X|^{p}>\frac{y}{2^{p-1}}-|-\mu|^{p}\right) d y \\
& =\int_{-|-\mu|^{p}}^{\infty} P\left(|X|^{p}>z\right) 2^{p-1} d z \\
& =\int_{0}^{\infty} P\left(|X|^{p}>z\right) 2^{p-1} d z \\
& =2^{p-1} E\left(|X|^{p}\right) .
\end{aligned}
$$

\subsubsection{Prova da Proposição 5, Item 2}

Os principais passos são os mesmos de Kerkyacharian e Picard (2004), para provar seu resultado (65) p.1086. Entretanto, como os erros são correlacionados, é preciso fazer as seguintes adaptações na 
página 1087 de Kerkyacharian e Picard (2004). Primeiro, observe que dados $\left(X_{1}=x_{1}, \ldots, X_{n}=x_{n}\right)$, $n^{-1} \sum_{t=1}^{n} \psi_{j, k}\left(G\left(X_{t}\right)\right) \epsilon_{t}$ é normalmente distribuído com média zero e

$$
\begin{gathered}
\operatorname{Var}\left(n^{-1} \sum_{t=1}^{n} \psi_{j, k}\left(G\left(x_{t}\right)\right) \epsilon_{t}\right)=E\left(\left(n^{-1} \sum_{t=1}^{n} \psi_{j, k}\left(G\left(x_{t}\right)\right) \epsilon_{t}\right)^{2}\right) \\
=n^{-2} \sum_{r=1}^{n} \sum_{s=1}^{n} \psi_{j, k}\left(G\left(x_{r}\right)\right) \psi_{j, k}\left(G\left(x_{s}\right)\right) E\left(\epsilon_{r} \epsilon_{s}\right)=n^{-2} \sum_{r=1}^{n} \sum_{s=1}^{n} \psi_{j, k}\left(G\left(x_{r}\right)\right) \psi_{j, k}\left(G\left(x_{s}\right)\right) \sigma_{r, s},
\end{gathered}
$$

Agora, seja $Z_{r}=\sum_{s=1}^{n} \psi_{j, k}\left(G\left(X_{r}\right)\right) \psi_{j, k}\left(G\left(X_{s}\right)\right) \sigma_{r, s}$. Tem-se que estas variáveis são limitadas

$$
\left|Z_{r}\right| \leq 2^{j}|| \psi||_{\infty}^{2} \sum_{s=1}^{\infty}\left|\sigma_{r, s}\right|<M<\infty
$$

porque, pela desigualdade de Davydov (DOUKHAN, 1994, Teorema 3, p. 9),

$$
\sum_{s=1}^{\infty}\left|\sigma_{r, s}\right| \leq \sum_{s=1}^{\infty} 8 \alpha_{\epsilon, s}^{1 / m}\left(E\left(\left|\epsilon_{r}\right|^{p}\right)\right)^{1 / p}\left(E\left(\left|\epsilon_{s}\right|^{q}\right)\right)^{1 / q}
$$

para quaisquer $p, q, m \geq 1,1 / p+1 / q+1 / m=1$, e pode-se escolher $p, q, m$ tal que pela Suposição 6 ,

$$
\begin{aligned}
& \sum_{s=1}^{\infty} 8 \alpha_{\epsilon, s}^{1 / m}\left(E\left(\left|\epsilon_{r}\right|^{p}\right)\right)^{1 / p}\left(E\left(\left|\epsilon_{s}\right|^{q}\right)\right)^{1 / q} \\
& \quad \leq \sum_{s=1}^{\infty}(s+1)^{\delta(m-1)-2}\left(\alpha_{\epsilon, s}\right)^{1 / m}\left(E\left(\left|\epsilon_{r}\right|^{p}\right)\right)^{1 / p}\left(E\left(\left|\epsilon_{s}\right|^{q}\right)\right)^{1 / q} \\
& \quad=\left(E\left(\left|\epsilon_{r}\right|^{p}\right)\right)^{1 / p}\left(E\left(\left|\epsilon_{s}\right|^{q}\right)\right)^{1 / q} \sum_{s=1}^{\infty}(s+1)^{c-2}\left(\alpha_{\epsilon, s}\right)^{\delta /(c+\delta)}<\infty .
\end{aligned}
$$

Também,

$$
E\left(Z_{r}\right)=\sum_{s=1}^{n} E\left(\psi_{j, k}\left(G\left(X_{r}\right)\right) \psi_{j, k}\left(G\left(X_{s}\right)\right)\right) \sigma_{r, s}=E\left(\psi_{j, k}^{2}\left(G\left(X_{r}\right)\right)\right) \sigma^{2}=1,
$$

e $\operatorname{Var}\left(Z_{r}\right)=E\left(Z_{r}^{2}\right)-1$, onde

$$
\begin{gathered}
E\left(Z_{r}^{2}\right)=E\left(\psi_{j, k}^{2}\left(G\left(X_{r}\right)\right) \sum_{l=1}^{n} \sum_{m=1}^{n} \psi_{j, k}\left(G\left(X_{l}\right)\right) \psi_{j, k}\left(G\left(X_{m}\right)\right) \sigma_{r, l} \sigma_{r, m}\right) \\
=E\left(\psi_{j, k}^{2}\left(G\left(X_{r}\right)\right) \sum_{l=1}^{n} \psi_{j, k}^{2}\left(G\left(X_{l}\right)\right) \sigma_{r, l}^{2}\right)=E\left(\psi_{j, k}^{4}\left(G\left(X_{r}\right)\right)+\psi_{j, k}^{2}\left(G\left(X_{r}\right)\right) \sum_{\substack{l=1 \\
l \neq r}}^{n} \psi_{j, k}^{2}\left(G\left(X_{l}\right)\right) \sigma_{r, l}^{2}\right)
\end{gathered}
$$




$$
=\int_{a}^{b} \psi_{j, k}^{4}(G(x)) g(x) d x+\sum_{\substack{l=1 \\ l \neq r}}^{n} \sigma_{r, l}^{2} \leq 2^{2 j}\|\psi\|_{\infty}^{4}+\sum_{\substack{l=1 \\ l \neq r}}^{\infty} \sigma_{r, l}^{2}=2^{2 j}\|\psi\|_{\infty}^{4}+\sigma_{r},
$$

pela Suposição 6, onde $\sigma_{r}=\sum_{\substack{l=1 \\ l \neq r}}^{\infty} \sigma_{r, l}^{2}$. Portanto, $Z_{r}$ são variáveis aleatórias independentes mas não são identicamente distribuídas.

Seja $\sigma_{*}=\sup _{r} \sigma_{r}$ e

$$
b_{n}^{2}=\sum_{r=1}^{n} E\left(\left(Z_{r}-1\right)^{2}\right)=\sum_{r=1}^{n} E\left(Z_{r}^{2}\right)-n .
$$

Agora, usando a desigualdade de Hoeffding Härdle et al. (1998, Corolário C.1, item (i)), temos

$$
\begin{gathered}
P\left(n^{-1}\left|\sum_{i=1}^{n}\left(Z_{i}-1\right)\right|>\alpha\right)=P\left(\left|\sum_{i=1}^{n}\left(Z_{i}-1\right)\right|>n \alpha\right) \\
\leq 2 \exp \left(-\frac{n^{2} \alpha^{2}}{2\left(\sum_{i=1}^{n} E\left(Z_{i}^{2}\right)-n+n \alpha M / 3\right)}\right) \leq 2 \exp \left(-\frac{n^{2} \alpha^{2}}{2\left(\sum_{i=1}^{n} E\left(Z_{i}^{2}\right)+n \alpha M\right)}\right) \\
\leq 2 \exp \left(-\frac{n^{2} \alpha^{2}}{2\left(n 2^{2 j}\|\psi\|_{\infty}^{4}+n \sigma_{*}+n \alpha M\right)}\right) \leq 2 \exp \left(-\frac{2 n \alpha^{2}}{C_{r} 2^{2 j}}\right) \\
\leq 2 \exp \left(-\frac{2 n \alpha^{2}}{C_{r}} \log n\right)=2 n^{-2 \alpha^{2} / C_{r}},
\end{gathered}
$$

se $2^{j} \leq \sqrt{n / \log n}=n_{3}$, onde $C_{r}=4\left(\|\psi\|_{\infty}^{4}+\sigma_{*}+\|\psi\|_{\infty}^{4} \sum_{s=1}^{\infty} \sigma_{r, s}\right.$.

\subsection{Procedimento Usando Ondaletas Adaptativas de Haar}

Considere o modelo (2.10) e as seguintes suposições:

A1 a função densidade $g$ é contínua e positiva no interior de um intervalo compacto $I: g(x) \geq c>0$ para todo $x \in \stackrel{\circ}{I}, g(x)=0$ para todo $x \in \mathbb{R} \backslash \stackrel{\circ}{I}$;

A2 a função $f$ é Hölder contínua em $I$, com expoente $1 / 2<\beta<1$, isto é $f \in \Lambda^{\beta}(L), \beta>1 / 2$;

A3 a função $f$ é de variação total limitada sobre o intervalo $I$;

A4 $E\left(\left|\epsilon_{t}\right|^{p}\right) \leq C^{p}(p !)^{k}$ para todo $p \geq 2$ e apropriadamente escolhidos $C<\infty$ e $k \geq 0$;

A5 o termo de erro $\left\{\epsilon_{t}\right\}_{t=1}^{n}$ provém de um processo estocástico fracamente estacionário com $E\left(\epsilon_{t}\right)=0$, $\operatorname{Var}\left(\epsilon_{t}\right)=\sigma_{\epsilon}^{2}<\infty$, e $\sum_{u}\left|\operatorname{Cov}\left(\epsilon_{t}, \epsilon_{t+u}\right)\right|=\sum_{u}\left|\gamma_{\epsilon}(u)\right|<K<\infty$ para todo $t$ e para todo $n ;$

A6 o limiar $\lambda_{j, k}$ é tal que

$$
\sigma_{j, k} \sqrt{2 \log \left(\# \mathcal{J}_{n}\right)} \leq \lambda_{j, k} \leq \frac{C}{\sqrt{n}} \sqrt{\log n},
$$

para uma constante positiva $C$, onde $\sigma_{j, k}$ é a variância condicional dos coeficientes empíricos de ondaletas $\hat{d}_{j, k}$, dado o valor das variáveis regressoras $X_{1}, \ldots, X_{n}$; 
A7 para que o estimador não-linear de ondaletas não seja pior que o linear, o valor de $\alpha$ em $\mathcal{J}_{n}$ deve ser tal que $0<\alpha<2 \beta /(2 \beta+1)$.

A8 $\left\{\epsilon_{t}\right\}_{t=1}^{n}$ é $\alpha$-mixing $\operatorname{com} \alpha(s) \leq C \exp (-b|s|)$.

Estas suposições são usuais para ondaletas adaptativas (DELOUILLE, 2002), exceto pela restrição na covariância na suposição A5 e pelas suposições A4 e A8. A suposição A4 não é tão forte quanto parece; de fato, muitas distribuições encontradas em livros-texto a satisfazem para escolhas apropriadas de $k$ (NEUMANN; SACHS, 1995). Estas suposições são necessárias para lidarmos com a dependência no termo de erro e são suficientes para que o seguinte teorema continue válido:

Teorema 5 (DElOUILle, 2002, Teorema 3.2.3) Sob as suposições A1 a A8,

$$
\sup _{f \in \Lambda^{\beta}(L)}\left(E\|\hat{f}-f\|_{L_{2}(d \hat{G})}^{2}\right)=O\left((\log (n) / n)^{2 \beta /(2 \beta+1)}\right) .
$$

Como a base formada por (2.13) e (2.14) é baseada em uma amostra finita, ela não pode gerar uma representação infinito-dimensional de uma função $f$ arbitrária. Portanto, algumas vezes, a função $f(x)$ de referência será substituída por sua projeção

$$
f_{J}(x)=\sum_{k=1}^{2^{j_{0}}} s_{j_{0}, k} \check{\phi}_{j_{0}, k}(x)+\sum_{j=j_{0}}^{J-1} \sum_{k=1}^{2^{j}} d_{j, k} \check{\psi}_{j, k}(x),
$$

A prova deste teorema é longa e detalhada, a despeito de não considerar nem uma classe de Hölder por partes, nem tamanhos $n$ de amostra que não sejam potência de dois. A prova usa diversos resultados intermediários constantes em Delouille (2002) e não serão completamente reproduzidos aqui. Entretanto, será apresentado um esboço da prova completa, juntamente com as modificações feitas por se considerar erros estacionários correlacionados.

\subsubsection{Melhor Estimador Linear}

Considere um modelo intermediário:

$$
Y_{t}^{*}=f\left(q_{t}\right)+\epsilon_{t}^{\prime}, \quad t=1, \ldots, n,
$$

onde os quantis $q_{t}=G_{x}^{-1}(t / n)$, teóricos e igualmente espaçados (em $L_{2}(d G)$ ), constitutem o delineamento fixo $q_{1}, \ldots, q_{n}$, tal que $I_{j, k}^{\circ}=I_{j, k}$ para todo $j \leq J$. Também, $\epsilon_{1}^{\prime}, \ldots, \epsilon_{n}^{\prime}$ são variáveis aleatórias escolhidas tais que $\epsilon_{t}^{\prime}=\epsilon_{s}$ se e somente se $X_{(t)}=X_{s}, t, s=1, \ldots, n$. Da estacionariedade em A5, $\epsilon_{t}$ tem a mesma distribuição de $\epsilon_{t}^{\prime}$, para todo $t$, e a distribuição conjunta de $\epsilon_{1}, \ldots, \epsilon_{n}$ é a mesma de $\epsilon_{1}^{\prime}, \ldots, \epsilon_{n}^{\prime}$, para todo $n$ fixo. Por construção, esta última distribuição satisfaz a condição de consistência do teorema da extensão de Kolmogorov (DAVIDSON, 1994; MORETTIN, 1999) e, portanto, ela 
define um processo estocástico $\left\{\epsilon_{t}^{\prime}\right\}_{t=1}^{\infty}$. Na realidade, este processo pode ser não estacionário mas os demais aspectos de $\mathbf{A} \mathbf{5}$ permanecem válidos.

Os coeficientes de ondaletas baseados no modelo (5.6) são

$$
\hat{d}_{j, k}^{*}=n^{-1} \sum_{t=1}^{n} Y_{t}^{*} \check{\psi}_{j, k}^{\circ}\left(q_{t}\right),
$$

e similarmente para $\hat{s}_{j_{0}, k}^{*}$

Claramente, um estimador baseado em $X_{1}, \ldots, X_{n}$ não pode ser melhor do que um baseado em $q_{1}, \ldots, q_{n}$. Uma referência usual na classe de Hölder considerada é o estimador linear de ondaletas

$$
\hat{f}^{l i n}=\sum_{k=1}^{2^{j_{0}}} \hat{s}_{j_{0}, k}^{*} \check{\phi}_{j_{0}, k}^{\circ}(x)+\sum_{j=j_{0}}^{j_{1}} \sum_{k=1}^{2^{j}} \hat{d}_{j, k}^{*} \check{\psi}_{j, k}^{\circ}(x),
$$

onde $j_{1}$ é algum nível de corte. Como a base de ondaletas é ortogonal, a correlação do erro não tem influência na decomposição do risco:

$$
\begin{aligned}
E\left\|\hat{f}^{l i n}(x)-f(x)\right\|_{L_{2}(d G)}^{2}= & \sum_{k} E\left|\hat{s}_{j_{0}, k}^{*}-s_{j_{0}, k}^{\circ}\right|^{2}+\sum_{j=j_{0}}^{j_{1}} \sum_{k} E\left|\hat{d}_{j, k}^{*}-d_{j, k}^{\circ}\right|^{2} \\
& +\sum_{j \geq j_{1}+1} \sum_{k}\left|d_{j, k}^{\circ}\right|^{2} \\
= & S_{1}+S_{2}+S_{3} .
\end{aligned}
$$

Sob as suposições A1 a A3, pela Proposição 3.6.1 em Delouille (2002), $S 3 \leq C 2^{-2 \beta j_{1}}$. Para $S 1+S 2$, é válida a seguinte

Proposição 6 Sob as suposições $\mathbf{A} 1$ a $\mathbf{A 3}$ e $\mathbf{A 5}$, na decomposição (5.8) do risco,

$$
S_{1}+S_{2} \leq \frac{2 \times 4^{j_{0}}+4^{j_{1}+1}}{3} \frac{\sum_{u}\left|\gamma_{\epsilon}(u)\right|}{n}+2^{j_{1}+1} o\left(n^{-1}\right)
$$

Para um $j_{0}$ fixo, o nível de corte que minimiza o risco é tal que os dois termos $C 2^{-2 \beta j_{1}}$ e $4^{j_{1}+1} \sum_{u}\left|\gamma_{\epsilon}(u)\right| /(3 n)$ são de mesma ordem. Isto é

$$
2^{-j_{1}^{o p t}}=C\left(\sum_{u}\left|\gamma_{\epsilon}(u)\right| / n\right)^{1 /(2+2 \beta)}=O\left(n^{-1 /(2+2 \beta)}\right) .
$$

Substituindo este resultado em (5.8), quando $2^{j_{1}^{\text {opt }}}=O\left(n^{1 /(2 \beta+2)}\right)$, o risco

$$
E\left\|\hat{f}^{l i n}(x)-f(x)\right\|_{L_{2}(d G)}^{2}=O\left(n^{-2 \beta /(2 \beta+2)}\right) .
$$


Essa taxa de convergência é um pouco mais lenta do que se os erros fossem i.i.d. onde, em tal caso, o melhor estimador linear de ondaletas é ótimo do ponto de vista da taxa de convergência minimax (BIERENS, 1983; BENTKUs, 1985). Note que a taxa de convergência do melhor estimador linear reside entre aqueles estimadores citados no Teorema 5. Além desse decaimento mais lento, outra dificuldade com o estimador linear é que não é fácil estimar $\beta$ em $2^{j_{1}^{\text {opt }}}$.

\subsubsection{Estimador Não-Linear}

Considere (2.20) e (5.5), adicione e subtraia $\sum_{j, k} d_{j, k}^{\circ} \check{\psi}_{j, k}(x)$ à diferença $\hat{f}(x)-f_{J}(x)$, use a ortogonalidade da base de ondaletas não balanceada de Haar e tome esperanças para obter

$$
\begin{aligned}
E & \left\|\hat{f}-f_{J}(x)\right\|_{L_{2}(d \hat{G})}^{2} \\
\leq & 2\left(\sum_{k} E\left(\left(\hat{s}_{j_{0}, k}-s_{j_{0}, k}\right)^{2}\right)+\sum_{j, k} E\left(\left(\eta_{H}\left(\hat{d}_{j, k}, \lambda_{j, k}\right)-d_{j, k}^{\circ}\right)^{2}\right)\right. \\
& \left.\quad+\sum_{j, k} E\left(\left(d_{j, k}^{\circ}-d_{j, k}\right)^{2}\right)\right) \\
= & T_{1}+T_{2}+T_{3} .
\end{aligned}
$$

A estratégia é provar que ambos $T_{1}$ e $T_{3}$ são $o\left(n^{-2 \beta /(2 \beta+1)}\right)$, e que $T_{2}=O\left((\log (n) / n)^{2 \beta /(2 \beta+1)}\right)$. Usando a monotonicidade das funções de limiar $\eta_{H}$ (ou $\eta_{S}$ ) em seu primeiro argumento, o termo $T_{2}$ pode ser limitado por

$$
E\left(\left(\eta_{H}\left(\hat{d}_{j, k}, \lambda_{j, k}\right)-d_{j, k}^{\circ}\right)^{2}\right) \leq 2 E\left(\left(\hat{d}_{j, k}-\hat{d}_{j, k}^{*}\right)^{2}\right)+2 E\left(\left(\eta_{H}\left(\hat{d}_{j, k}^{*}, \lambda_{j, k}\right)-d_{j, k}^{\circ}\right)^{2}\right) .
$$

Com esta decomposição, para $T_{2}$ é suficiente provar que

$$
\begin{gathered}
T_{2,1}=\sum_{(j, k) \in \mathcal{J}_{n}} E\left(\left(\hat{d}_{j, k}-\hat{d}_{j, k}^{*}\right)^{2}\right)=o\left(n^{-2 \beta /(2 \beta+1)}\right) \\
T_{2,2}=\sum_{j, k} E\left(\left(\eta_{H}\left(\hat{d}_{j, k}^{*}, \lambda_{j, k}\right)-d_{j, k}^{\circ}\right)^{2}\right)=O\left(\left(\frac{\log n}{n}\right)^{\frac{2 \beta}{2 \beta+1}}\right) .
\end{gathered}
$$

Considerando $T_{1}$, não é difícil ver que

$$
E\left(\left(\hat{s}_{j_{0}, k}-s_{j_{0}, k}\right)^{2}\right)=\operatorname{Var}\left(\hat{s}_{j_{0}, k}\right)=E\left(\operatorname{Var}\left(\hat{s}_{j_{0}, k} \mid X_{1}, \ldots, X_{n}\right)\right) .
$$

Também é válido o seguinte 
Proposição 7 Sob a suposição A5, o coeficiente de ondaleta em (2.19) é tal que

$$
E\left(\operatorname{Var}\left(\hat{s}_{j_{0}, k} \mid X_{1}, \ldots, X_{n}\right)\right)<\frac{C}{n 2^{j_{0}}} .
$$

Então, o resultado da Proposição 3.6.2 em Delouille (2002) permanece válido:

$$
T_{1}=\sum_{k} E\left(\left(\hat{s}_{j_{0}, k}-s_{j_{0}, k}\right)^{2}\right)<\frac{2^{j_{0}} C}{n 2^{j_{0}}}=C n^{-1}=o\left(n^{-2 \beta /(2 \beta+1)}\right) .
$$

A Proposição 3.3.6 em Delouille (2002) prova o resultado para $T_{3}$ e é baseada na Proposição 3.3.5 em Delouille (2002) que também é usada na prova dos resultados para $T_{2,1}$. Entretanto, essas provas são influenciadas pela correlação dos erros apenas através de alguns resultados das Proposições 3.3.1 a 3.3.3 em Delouille (2002).

Nessas proposições, apenas a Proposição 3.3.1(ii), Proposição 3.3.2 e Proposição 3.3.3(iii) são influenciadas pela correlação dos erros. Os outros ou são influenciados apenas pelos $X$ independentes ou envolvem esperanças simples, sem termos quadráticos.

O resultado na Proposição 3.3.3(iii) em Delouille (2002) refere-se a $\operatorname{Var}\left(\hat{d}_{j, k}^{*}\right)$ e seu equivalente, no caso de erros estationários correlacionados, é dado por (5.9), na prova da Proposição 6, nesta tese.

Ainda considerando o caso de erros estacionários correlacionados, o equivalente à Proposição 3.3.1(ii) e Proposição 3.3.2, ambas em Delouille (2002), são respectivamente dados por:

Proposição 8 O coeficiente definido por (2.17) é tal que, uniformemente sobre $(j, k) \in \mathcal{J}_{n}$,

$$
\operatorname{Var}\left(\hat{d}_{j, k}^{\#}\right)=n^{-1}\left(\sigma_{\epsilon}^{2}+\operatorname{Var}\left(f\left(X_{1}\right) \check{\psi}_{j, k}^{\circ}\left(X_{1}\right)\right)\right)
$$

Proposição 9 Uniformemente sobre $(j, k) \in \mathcal{J}_{n}$, os coeficientes

$$
\rho_{j, k}=n^{-1} \sum_{t=1}^{n} \epsilon_{t} \check{\psi}_{j, k}\left(X_{t}\right) \quad e \quad \rho_{j, k}^{*}=n^{-1} \sum_{t=1}^{n} \epsilon_{t}^{\prime} \check{\psi}_{j, k}^{\circ}\left(q_{t}\right)
$$

satisfazem

$$
E\left(\left(\rho_{j, k}-\rho_{j, k}^{*}\right)^{2}\right)=O\left(\frac{2^{j}}{n^{3 / 2}}\right)
$$

Falta provar o resultado para o termo $T_{2,2}$. Para isso uma aproximação gaussiana é usada. Considere o modelo (5.6) e suponha que as suposições A1 a A8 seja satisfeitas. Assim, de acordo com Neumann e Sachs (1995), mesmo quando os erros são correlacionados, o Lema 3.4.1, a Proposição 3.4.2 e o Teorema 3.4.3 em Delouille (2002), permanecem válidos. Esses resultados, juntamente com um limiar adequadamente escolhido provêm a desejada taxa de convergência para o termo $T_{2,2}$ (como em Delouille (2002), Seção 3.4.3). 


\subsection{Provas da Seção 5.3}

\subsubsection{Prova da Proposição 6}

Primeiramente, note que a Proposição 3.3.3(i) em Delouille (2002) permanece válida uma vez que as correlações dos erros não influenciam esperanças simples (sem termos quadráticos). Assim,

$$
\left|E\left(\hat{d}_{j, k}^{*}-d_{j, k}^{\circ}\right)\right| \leq n^{-1} 2^{j / 2} V\left(f ; q_{(k-1) n_{j}}, q_{k n_{j}}\right),
$$

onde $n_{j}=n 2^{-j}$ e a variação total

$$
V\left(f ; q_{(k-1) n_{j}}, q_{k n_{j}}\right)=\sup \sum_{i=1}^{l}\left|f\left(t_{i}\right)-f\left(t_{i-1}\right)\right|,
$$

com o supremo sendo avaliado sobre todas as partições $\left\{t_{0}, \ldots, t_{l}\right\}$ do intervalo $\left[q_{(k-1) n_{j}}, q_{k n_{j}}\right]$. Logo,

$$
\left(E\left(\hat{d}_{j, k}^{*}-d_{j, k}^{\circ}\right)\right)^{2} \leq n^{-2} 2^{j} V^{2}\left(f ; q_{(k-1) n_{j}}, q_{k n_{j}}\right)=C / n^{2}=o\left(n^{-1}\right) .
$$

Por outro lado, a Proposição 3.3.3(iii) também em Delouille (2002), deve ser modificada para

$$
\begin{aligned}
\operatorname{Var}\left(\hat{d}_{j, k}^{*}\right) & =\operatorname{Var}\left(n^{-1} \sum_{t=1}^{n} \epsilon_{t}^{\prime} \check{\psi}_{j, k}^{\circ}\left(q_{t}\right)\right) \\
& =\operatorname{Cov}\left(n^{-1} \sum_{t=1}^{n} \epsilon_{t}^{\prime} \check{\psi}_{j, k}^{\circ}\left(q_{t}\right), n^{-1} \sum_{r=1}^{n} \epsilon_{r}^{\prime} \check{\psi}_{j, k}^{\circ}\left(q_{r}\right)\right) \\
& =n^{-2} \sum_{t=1}^{n} \sum_{r=1}^{n} \check{\psi}_{j, k}^{\circ}\left(q_{t}\right) \check{\psi}_{j, k}^{\circ}\left(q_{r}\right) \operatorname{Cov}\left(\epsilon_{t}^{\prime}, \epsilon_{r}^{\prime}\right) .
\end{aligned}
$$

Combinando estes dois últimos resultados e usando a suposição A5,

$$
\begin{aligned}
& E\left(\hat{d}_{j, k}^{*}-d_{j, k}^{\circ}\right)^{2} \\
& =\operatorname{Var}\left(\hat{d}_{j, k}^{*}-d_{j, k}^{\circ}\right)+\left(E\left(\hat{d}_{j, k}^{*}-d_{j, k}^{\circ}\right)\right)^{2} \\
& \leq \operatorname{Var}\left(\hat{d}_{j, k}^{*}\right)+o\left(n^{-1}\right) \\
& =n^{-2} \sum_{t=1}^{n} \sum_{r=1}^{n} \check{\psi}_{j, k}^{\circ}\left(q_{t}\right) \check{\psi}_{j, k}^{\circ}\left(q_{r}\right) \operatorname{Cov}\left(\epsilon_{t}^{\prime}, \epsilon_{r}^{\prime}\right)+o\left(n^{-1}\right) \\
& \leq n^{-2} \sum_{t=1}^{n} \sum_{r=1}^{n}\left|\check{\psi}_{j, k}^{\circ}\left(q_{t}\right)\right|\left|\check{\psi}_{j, k}^{\circ}\left(q_{r}\right)\right|\left|\operatorname{Cov}\left(\epsilon_{t}^{\prime}, \epsilon_{r}^{\prime}\right)\right|+o\left(n^{-1}\right) \\
& \leq n^{-2} \sum_{t=1}^{n} \sum_{r=1}^{n} 2^{j}\left|\operatorname{Cov}\left(\epsilon_{t}^{\prime}, \epsilon_{r}^{\prime}\right)\right|+o\left(n^{-1}\right)
\end{aligned}
$$




$$
\begin{aligned}
& =2^{j} n^{-2} \sum_{t=1}^{n} \sum_{r=1}^{n}\left|\gamma_{\epsilon}\right| t-r||+o\left(n^{-1}\right) \\
& =2^{j} n^{-2} \sum_{t=1}^{n} \sum_{u=-(n-1)}^{n-1}\left|\gamma_{\epsilon}\right| u||+o\left(n^{-1}\right) \\
& =\frac{2^{j}}{n} \sum_{u=-(n-1)}^{n-1}\left|\gamma_{\epsilon}\right| u||+o\left(n^{-1}\right) .
\end{aligned}
$$

Substitutindo este resultado na decomposição do risco (5.8),

$$
\begin{aligned}
S_{1}+S_{2} & \leq 2^{j_{0}}\left(\frac{2^{j_{0}}}{n} \sum_{u}\left|\gamma_{\epsilon}\right| u||+o\left(n^{-1}\right)\right)+\sum_{j=j_{0}}^{j_{1}} 2^{j}\left(\frac{2^{j}}{n} \sum_{u}\left|\gamma_{\epsilon}\right| u||+o\left(n^{-1}\right)\right) \\
& =\frac{4^{j_{0}}}{n} \sum_{u}\left|\gamma_{\epsilon}\right| u||+2^{j_{0}} o\left(n^{-1}\right)+\sum_{j=j_{0}}^{j_{1}} \frac{4^{j}}{n} \sum_{u}\left|\gamma_{\epsilon}\right| u||+\sum_{j=j_{0}}^{j_{1}} 2^{j} o\left(n^{-1}\right) \\
& =\frac{2 \times 4^{j_{0}}+4^{j_{1}+1}}{3} \frac{\sum_{u}\left|\gamma_{\epsilon}\right| u||}{n}+2^{j_{1}+1} o\left(n^{-1}\right) .
\end{aligned}
$$

\subsubsection{Prova da Proposição 7}

Primeiramente note que,

$$
\begin{aligned}
& \operatorname{Var}\left(\hat{s}_{j_{0}, k} \mid X_{1}, \ldots, X_{n}\right) \\
& =\operatorname{Var}\left(n^{-1} \sum_{t=1}^{n} Y_{t} \check{\phi}_{j_{0}, k}\left(X_{t}\right) \mid X_{1}, \ldots, X_{n}\right) \\
& =\operatorname{Var}\left(n^{-1} \sum_{t=1}^{n}\left(f\left(X_{t}\right)+\epsilon_{t}\right) \check{\phi}_{j_{0}, k}\left(X_{t}\right) \mid X_{1}, \ldots, X_{n}\right) \\
& =\operatorname{Var}\left(n^{-1} \sum_{t=1}^{n} \epsilon_{t} \check{\phi}_{j_{0}, k}\left(X_{t}\right) \mid X_{1}, \ldots, X_{n}\right) \\
& =\operatorname{Cov}\left(n^{-1} \sum_{t=1}^{n} \epsilon_{t} \check{\phi}_{j_{0}, k}\left(X_{t}\right), n^{-1} \sum_{r=1}^{n} \epsilon_{r} \check{\phi}_{j_{0}, k}\left(X_{r}\right) \mid X_{1}, \ldots, X_{n}\right) \\
& =n^{-2} \sum_{t=1}^{n} \sum_{r=1}^{n} \check{\phi}_{j_{0}, k}\left(X_{t}\right) \check{\phi}_{j_{0}, k}\left(X_{r}\right) \operatorname{Cov}\left(\epsilon_{t}, \epsilon_{r}\right) .
\end{aligned}
$$

Então, por independência dos $X$, definição (2.13), e suposição A5,

$$
E\left(\operatorname{Var}\left(\hat{s}_{j_{0}, k} \mid X_{1}, \ldots, X_{n}\right)\right)
$$




$$
\begin{aligned}
& =n^{-2} \sum_{t=1}^{n} \sum_{r=1}^{n} E\left(\check{\phi}_{j_{0}, k}\left(X_{t}\right) \check{\phi}_{j_{0}, k}\left(X_{r}\right)\right) \gamma_{\epsilon}(|t-r|) \\
& =n^{-2} \sum_{t=1}^{n} \sum_{r=1}^{n} 2^{j_{0}} E\left(1_{j_{0}, k}\left(X_{t}\right)\right) E\left(1_{j_{0}, k}\left(X_{r}\right)\right) \gamma_{\epsilon}(|t-r|) \\
& =n^{-2} \sum_{t=1}^{n} \sum_{r=1}^{n} 2^{j_{0}} 2^{-j_{0}} 2^{-j_{0}} \gamma_{\epsilon}(|t-r|) \\
& \leq n^{-2} \sum_{u=-(n-1)}^{n-1} \gamma_{\epsilon}(|u|) \sum_{t=1}^{n} 2^{-j_{0}} \\
& <n^{-1} C 2^{-j_{0}}
\end{aligned}
$$

\subsubsection{Prova da Proposição 8}

Usando a definição (2.17):

$$
\begin{aligned}
\operatorname{Var}\left(\hat{d}_{j, k}^{\#}\right) & =\operatorname{Var}\left(n^{-1} \sum_{t=1}^{n} Y_{t} \check{\psi}_{j, k}^{\circ}\left(X_{t}\right)\right) \\
& =\operatorname{Var}\left(n^{-1} \sum_{t=1}^{n}\left(f\left(X_{t}\right)+\epsilon_{t}\right) \check{\psi}_{j, k}^{\circ}\left(X_{t}\right)\right) \\
& =n^{-2} \operatorname{Var}\left(\sum_{t=1}^{n} f\left(X_{t}\right) \check{\psi}_{j, k}^{\circ}\left(X_{t}\right)+\sum_{r=1}^{n} \epsilon_{r} \check{\psi}_{j, k}^{\circ}\left(X_{r}\right)\right) .
\end{aligned}
$$

Como $\epsilon_{t}$ é independente de $X_{t}$ para todo $t$, e $X_{i}$ é independente de $X_{j}$ para todo $i \neq j$, então

$$
\begin{aligned}
\operatorname{Var}\left(\hat{d}_{j, k}^{\#}\right)= & n^{-2}\left[\operatorname{Var}\left(\sum_{t=1}^{n} f\left(X_{t}\right) \check{\psi}_{j, k}^{\circ}\left(X_{t}\right)\right)+\operatorname{Var}\left(\sum_{r=1}^{n} \epsilon_{r} \check{\psi}_{j, k}^{\circ}\left(X_{r}\right)\right)\right] \\
= & n^{-1} \operatorname{Var}\left(f\left(X_{1}\right) \check{\psi}_{j, k}^{\circ}\left(X_{1}\right)\right)+n^{-2} E\left[\left(\sum_{r=1}^{n} \epsilon_{r} \check{\psi}_{j, k}^{\circ}\left(X_{r}\right)\right)^{2}\right] \\
& -n^{-2}\left[E\left(\sum_{r=1}^{n} \epsilon_{r} \check{\psi}_{j, k}^{\circ}\left(X_{r}\right)\right)\right]^{2} \\
= & n^{-1} \operatorname{Var}\left(f\left(X_{1}\right) \check{\psi}_{j, k}^{\circ}\left(X_{1}\right)\right) \\
& +n^{-2} \sum_{r=1}^{n} \sum_{s=1}^{n} E\left(\epsilon_{r} \epsilon_{s} \check{\psi}_{j, k}^{\circ}\left(X_{r}\right) \check{\psi}_{j, k}^{\circ}\left(X_{s}\right)\right)-0 \\
= & n^{-1} \operatorname{Var}\left(f\left(X_{1}\right) \check{\psi}_{j, k}^{\circ}\left(X_{1}\right)\right) \\
& +n^{-2} \sum_{r=1}^{n} \sum_{s=1}^{n} \operatorname{Cov}\left(\epsilon_{r}, \epsilon_{s}\right) E\left(\check{\psi}_{j, k}^{\circ}\left(X_{r}\right) \check{\psi}_{j, k}^{\circ}\left(X_{s}\right)\right) .
\end{aligned}
$$


Novamente, pela independência dos $X$, ortonormalidade da base de ondaletas, e como para todo $t, j, k$ $E\left(\check{\psi}_{j, k}^{\circ}\left(X_{t}\right)\right)=0$, então

$$
\begin{aligned}
\operatorname{Var}\left(\hat{d}_{j, k}^{\#}\right)= & n^{-1} \operatorname{Var}\left(f\left(X_{1}\right) \check{\psi}_{j, k}^{\circ}\left(X_{1}\right)\right) \\
& +n^{-2} \sum_{r=1}^{n} \sum_{\substack{s=1 \\
s \neq r}}^{n} \operatorname{Cov}\left(\epsilon_{r}, \epsilon_{s}\right) E\left(\check{\psi}_{j, k}^{\circ}\left(X_{r}\right)\right) E\left(\check{\psi}_{j, k}^{\circ}\left(X_{s}\right)\right) \\
& +n^{-2} \sum_{t=1}^{n} \sigma_{\epsilon}^{2} E\left(\check{\psi}_{j, k}^{\circ}\left(X_{t}\right)^{2}\right) \\
= & n^{-1} \operatorname{Var}\left(f\left(X_{1}\right) \check{\psi}_{j, k}^{\circ}\left(X_{1}\right)\right)+\sigma_{\epsilon}^{2} / n .
\end{aligned}
$$

\subsubsection{Prova da Proposição 9}

Defina

$$
\hat{\rho}_{j, k}^{\#}=n^{-1} \sum_{t=1}^{n} \epsilon_{t} \check{\psi}_{j, k}^{\circ}\left(X_{t}\right)
$$

como um coeficiente intermediário e use a desigualdade (numérica) de Hölder para escrever

$$
E\left(\left(\rho_{j, k}-\rho_{j, k}^{*}\right)^{2}\right) \leq 2\left[E\left(\left(\rho_{j, k}^{*}-\rho_{j, k}^{\#}\right)^{2}\right)+E\left(\left(\rho_{j, k}-\rho_{j, k}^{\#}\right)^{2}\right)\right]
$$

e mostre que ambos os termos são de ordem $\sigma_{\epsilon}^{2} O\left(2^{j} / n^{3 / 2}\right)$.

\section{Prova para o Primeiro Termo}

Denotando $n_{j}=n 2^{-j}$,

$$
\begin{aligned}
& E\left(\left(\rho_{j, k}^{\#}-\rho_{j, k}^{*}\right)^{2}\right) \\
& =n^{-2} E\left(\sum_{t=1}^{n} \epsilon_{t}\left(\check{\psi}_{j, k}^{\circ}\left(X_{(t)}\right)-\check{\psi}_{j, k}^{\circ}\left(q_{t}\right)\right)\right)^{2} \\
& =n^{-2} E \sum_{r=1}^{n} \sum_{s=1}^{n} \epsilon_{r} \epsilon_{s}\left(\check{\psi}_{j, k}^{\circ}\left(X_{(r)}\right)-\check{\psi}_{j, k}^{\circ}\left(q_{r}\right)\right)\left(\check{\psi}_{j, k}^{\circ}\left(X_{(s)}\right)-\check{\psi}_{j, k}^{\circ}\left(q_{s}\right)\right),
\end{aligned}
$$

pela independência entre $\epsilon_{t}$ e $X_{t}$, para todo $t$. Fazendo $n_{j}=n 2^{-j}$, a última expressão fica igual a

$$
\begin{aligned}
\frac{1}{n n_{j}} \sum_{r=1}^{n} \sum_{s=1}^{n} & \operatorname{Cov}\left(\epsilon_{r} \epsilon_{s}\right) E\left(\left(1_{j+1,2 k}^{\circ}\left(X_{(r)}\right)-1_{j+1,2 k}^{\circ}\left(q_{r}\right)-1_{j+1,2 k-1}^{\circ}\left(X_{(r)}\right)+1_{j+1,2 k-1}^{\circ}\left(q_{r}\right)\right)\right. \\
& \left.\times\left(1_{j+1,2 k}^{\circ}\left(X_{(s)}\right)-1_{j+1,2 k}^{\circ}\left(q_{s}\right)-1_{j+1,2 k-1}^{\circ}\left(X_{(s)}\right)+1_{j+1,2 k-1}^{\circ}\left(q_{s}\right)\right)\right)
\end{aligned}
$$


Usando $a(t)=1_{j+1,2 k}^{\circ}\left(X_{(t)}\right)-1_{j+1,2 k}^{\circ}\left(q_{t}\right)-1_{j+1,2 k-1}^{\circ}\left(X_{(t)}\right)+1_{j+1,2 k-1}^{\circ}\left(q_{t}\right)$ para abreviar a notação, a última expressão é igual a

$$
\begin{aligned}
& \frac{1}{n n_{j}} \sum_{t=1}^{n} \sigma_{\epsilon}^{2} E\left(a(t)^{2}\right)+\frac{1}{n n_{j}} \sum_{r=1}^{n} \sum_{\substack{s=1 \\
r \neq s}}^{n} \operatorname{Cov}\left(\epsilon_{r}, \epsilon_{s}\right) E(a(r) a(s)) \\
& \leq \frac{1}{n n_{j}} \sum_{t=1}^{n} \sigma_{\epsilon}^{2} E\left(a(t)^{2}\right)+\frac{1}{n n_{j}} \sum_{r=1}^{n} \sum_{\substack{s=1 \\
r \neq s}}^{n} \sigma_{\epsilon}^{2}|E(a(r) a(s))| \\
& =\frac{1}{n n_{j}} \sum_{t=1}^{n} \sigma_{\epsilon}^{2} E\left(a(t)^{2}\right)+\frac{1}{n n_{j}} \sum_{r=1}^{n} \sum_{\substack{s=1 \\
r \neq s}}^{n} \sigma_{\epsilon}^{2}|E(a(r)) E(a(s))|,
\end{aligned}
$$

Mas,

$$
\begin{aligned}
E(a(r))= & E\left(1_{j+1,2 k}^{\circ}\left(X_{(r)}\right)-1_{j+1,2 k}^{\circ}\left(q_{r}\right)\right) \\
& -E\left(1_{j+1,2 k-1}^{\circ}\left(X_{(r)}\right)-1_{j+1,2 k-1}^{\circ}\left(q_{r}\right)\right) \\
= & E\left(1_{j+1,2 k}^{\circ}\left(X_{(r)}\right)\right)-E\left(1_{j+1,2 k-1}^{\circ}\left(X_{(r)}\right)\right) \\
& -E\left(1_{j+1,2 k}\left(X_{(r)}\right)\right)+E\left(1_{j+1,2 k-1}\left(X_{(r)}\right)\right) \\
= & 0 .
\end{aligned}
$$

Portanto,

$$
E\left(\left(\rho_{j, k}^{\#}-\rho_{j, k}^{*}\right)^{2}\right)=\frac{\sigma_{\epsilon}^{2}}{n n_{j}} \sum_{t=1}^{n} E\left(a(t)^{2}\right) \leq 2 \sigma_{\epsilon}^{2}\left(V_{1}+V_{2}\right),
$$

onde

$$
V_{1}=\sum_{r=1}^{n} \frac{1}{n n_{j}} E\left(\left(1_{j+1,2 k}^{\circ}\left(X_{(r)}\right)-1_{j+1,2 k}^{\circ}\left(q_{r}\right)\right)^{2}\right)
$$

e

$$
V_{2}=\sum_{r=1}^{n} \frac{1}{n n_{j}} E\left(\left(1_{j+1,2 k-1}^{\circ}\left(X_{(r)}\right)-1_{j+1,2 k-1}^{\circ}\left(q_{r}\right)\right)^{2}\right) .
$$

Na prova da Proposição 3.3.2(i) em Delouille (2002), é provado que $V_{1}$ e $V_{2}$ são $O\left(1 /\left(n_{j} \sqrt{n}\right)\right.$ ).

\section{Prova para o Segundo Termo}

Para o segundo termo, com probabilidade 1:

$$
\begin{aligned}
\left(\rho_{j, k}-\rho_{j, k}^{\#}\right)^{2} \leq & 2 \frac{2^{j}}{n^{2}}\left[\sum_{t=1}^{n} \epsilon_{t}\left(1_{j+1,2 k}\left(X_{t}\right)-1_{j+1,2 k}^{\circ}\left(X_{t}\right)\right)\right]^{2}+ \\
& +2 \frac{2^{j}}{n^{2}}\left[\sum_{t=1}^{n} \epsilon_{t}\left(1_{j+1,2 k-1}\left(X_{t}\right)-1_{j+1,2 k-1}^{\circ}\left(X_{t}\right)\right)\right]^{2}
\end{aligned}
$$




$$
=2\left(S_{1}^{2}+S_{2}^{2}\right)
$$

Como $S_{2}$ é similar a $S_{1}$, apenas o seguinte resultado será apresentado. Como a partição aleatória é especificada pelas estatísticas de ordem, obtém-se:

$$
\begin{aligned}
E S_{1}^{2}= & \\
= & \frac{1}{n n_{j}} E\left(E\left(\left[\sum_{t=1}^{n} \epsilon_{t}\left(1_{j, k}\left(X_{t}\right)-1_{j, k}^{\circ}\left(X_{t}\right)\right)\right]^{2} \mid X_{(1)}, \ldots, X_{(n)}\right)\right) \\
= & \frac{1}{n n_{j}} E\left(E \left(\sum_{t=1}^{n} \epsilon_{t}^{2}\left(1_{j, k}\left(X_{t}\right)-1_{j, k}^{\circ}\left(X_{t}\right)\right)^{2}+\right.\right. \\
& \left.\left.\left.+\sum_{r=1}^{n} \sum_{\substack{s=1 \\
s \neq r}}^{n} \epsilon_{r} \epsilon_{s}\left(1_{j, k}\left(X_{r}\right)-1_{j, k}^{\circ}\left(X_{r}\right)\right)\left(1_{j, k}\left(X_{s}\right)-1_{j, k}^{\circ}\left(X_{s}\right)\right) \mid X_{(1)}, \ldots, X_{(n)}\right)\right)\right) \\
= & \frac{\sigma_{\epsilon}^{2}}{n_{j}} E\left(\left(1_{j, k}\left(X_{1}\right)-1_{j, k}^{\circ}\left(X_{1}\right)\right)^{2}\right)+ \\
& +\frac{1}{n n_{j}} E\left(\sum_{r=1}^{n} \sum_{\substack{s=1 \\
s \neq r}}^{n} \operatorname{Cov}\left(\epsilon_{r}, \epsilon_{s}\right) E\left(1_{j, k}\left(X_{r}\right)-1_{j, k}^{\circ}\left(X_{r}\right) \mid X_{(1)}, \ldots, X_{(n)}\right) \times\right. \\
& \left.\times E\left(1_{j, k}\left(X_{s}\right)-1_{j, k}^{\circ}\left(X_{s}\right) \mid X_{(1)}, \ldots, X_{(n)}\right)\right) \\
= & \frac{\sigma_{\epsilon}^{2}}{n_{j}} E\left(\left(1_{j, k}\left(X_{1}\right)-1_{j, k}^{\circ}\left(X_{1}\right)\right)^{2}\right)+ \\
& +\frac{1}{n n_{j}}\left(\sum_{r=1}^{n} \sum_{\substack{s=1 \\
s \neq r}}^{n} \operatorname{Cov}\left(\epsilon_{r}, \epsilon_{s}\right) E\left(1_{j, k}\left(X_{r}\right)-1_{j, k}^{\circ}\left(X_{r}\right)\right) E\left(1_{j, k}\left(X_{s}\right)-1_{j, k}^{\circ}\left(X_{s}\right)\right)\right) .
\end{aligned}
$$

Supondo por um momento que $E\left(1_{j, k}\left(X_{r}\right)-1_{j, k}^{\circ}\left(X_{r}\right)\right)=0$ para todo $r=1, \ldots, n$, então

$$
E S_{1}^{2}=\frac{\sigma_{\epsilon}^{2}}{n_{j}} E\left(\left(1_{j, k}\left(X_{1}\right)-1_{j, k}^{\circ}\left(X_{1}\right)\right)^{2}\right)
$$

Pela prova da Proposição 3.3.2(ii) e Lema 3.6.3, ambos em Delouille (2002), deste último resultado se obtém que, $E S_{1}^{2}=\sigma_{\epsilon}^{2} O\left(1 /\left(n_{j} \sqrt{n}\right)\right)$; o mesmo acontece com $E S_{2}^{2}$.

Colocando juntos os resultados para os dois termos,

$$
E\left(\left(\rho_{j, k}-\rho_{j, k}^{*}\right)^{2}\right)=O\left(\frac{2^{j}}{n^{3 / 2}}\right)
$$

e a Proposição 9 é provada. 
Para concluir a prova, o seguinte resultado ainda precisa ser provado:

$$
\begin{aligned}
E\left(1_{j, k}\left(X_{r}\right)-1_{j, k}^{\circ}\left(X_{r}\right)\right) & =E\left(1_{j, k}\left(X_{r}\right)\right)-E\left(1_{j, k}^{\circ}\left(X_{r}\right)\right) \\
& =P\left(X_{r} \in I_{j, k}\right)-P\left(X_{r} \in I_{j, k}^{\circ}\left(X_{r}\right)\right)=0 .
\end{aligned}
$$

Para isso, note que para todo $r$,

$$
\begin{aligned}
P\left(X_{r} \in I_{j, k}^{\circ}\left(X_{r}\right)\right) & =P\left(q_{(k-1) n_{j}} \leq X_{r}<q_{k n_{j}}\right) \\
& =\frac{k n_{j}-\left[(k-1) n_{j}\right]}{n} \\
& =\frac{n_{j}}{n} ;
\end{aligned}
$$

e

$$
\begin{aligned}
P\left(X_{r} \in I_{j, k}\right) & =P\left(X_{(k-1) n_{j}+1} \leq X_{r}<X_{k n_{j}+1}\right) \\
& =P\left(X_{(k-1) n_{j}+1} \leq X_{1}<X_{k n_{j}+1}\right) \\
& =P\left((k-1) n_{j}+1 \leq R_{1}<k n_{j}+1\right) \\
& =\frac{n_{j}}{n},
\end{aligned}
$$

como $P\left(R_{1}=s\right)=1 / n$ para todo $s$, e $R_{1}$ é o posto de $X_{1}$.

\subsection{Procedimento Usando Ondaletas Adaptativas Suaves (Lifting)}

Nesta seção serão tratadas funções suaves usando um esquema de lifting no contexto de ondaletas adaptativas. Serão desenvolvidos alguns resultados teóricos e serão comentados como eles podem ser usados para mostrar que um estimador via aplicação de limiares suaves alcança quase um risco "ideal" no domínio das ondaletas.

Como notado no Capítulo 2, Seção 2.5, além do problema de não se ter uma expressão analítica quando um passo de atualização é realizado, no caso de uma base bi-ortogonal de ondaletas em $L_{2}(d \hat{G})$ (ou em $L_{2}(d G)$ ), a igualdade de Parseval não é mais válida. Se fosse provado que a base de ondaletas resultante do esquema de lifting fosse uma base de Riesz do espaço $L_{2}(d \hat{G})$, então uma desigualdade poderia ser usada para avaliar o risco de algum estimador nesse espaço, com seu risco no domínio das ondaletas. Tal prova não é trivial por causa do espaçamento desigual e continua em aberto (DELOUILLE, 2002, p. 139). Devido a essas dificuldades, nossos resultados serão limitados ao domínio das ondaletas e condicionais a $\left\{X_{1}, \ldots, X_{n}\right\}$. 


\subsubsection{Alguns Resultados Sobre os Coeficientes de Ondaletas}

No modelo (2.10), seja

$$
d_{j, k}=n^{-1} \sum_{t=1}^{n} f\left(X_{t}\right) \tilde{\psi}_{j, k}\left(X_{t}\right)
$$

estimado por

$$
\hat{d}_{j, k}=n^{-1} \sum_{t=1}^{n} Y_{t} \tilde{\psi}_{j, k}\left(X_{t}\right)
$$

Como ferramenta, considere um modelo intermediário (5.6).

A função objetivo considerada é a projeção:

$$
f_{J}(x)=\sum_{k \in \mathcal{K}_{j_{0}}} s_{j_{0}, k}^{*} \phi_{j_{0}, k}(x)+\sum_{j=j_{0}}^{J-1} \sum_{k \in \mathcal{K}_{j}} d_{j, k}^{*} \psi_{j, k}(x),
$$

onde

$$
d_{j, k}^{*}=\left\langle f, \tilde{\psi}_{j, k}\right\rangle_{d \hat{G}}=n^{-1} \sum_{t=1}^{n} f\left(q_{t}\right) \tilde{\psi}_{j, k}\left(q_{t}\right),
$$

e similarmente para $s_{j_{0}, k}^{*}$. Também (DELOUILLE, 2002, eq. 4.20),

$$
\tilde{\psi}_{j, k}(x)=\sum_{l: \tilde{g}_{j l k} \neq 0} \tilde{g}_{j l k} \frac{1_{j+1, l}^{\circ}(x)}{\mu\left(I_{j+1, l}^{\circ}\right)^{1 / 2}},
$$

onde $\tilde{g}_{j l k}$ são as entradas da matriz de filtro $\tilde{G}_{j}$. Seja $d_{j, k}^{*}$ estimado por

$$
\hat{d}_{j, k}^{*}=n^{-1} \sum_{t=1}^{n} Y_{t}^{*} \tilde{\psi}_{j, k}\left(q_{t}\right)
$$

e tem-se a seguinte

Proposição 10 Considere o modelo (5.6) e as suposições sobre $G$ como no modelo (2.10). Sejam $d_{j, k}^{*}$ e $\hat{d}_{j, k}^{*}$ dados por (5.12) e (5.14), respectivamente. Também, sejam $d_{j, k}$ e $\hat{d}_{j, k}$ dados por (5.10) e (5.11), respectivamente. Então

$$
E\left(\left(\hat{d}_{j, k}^{*}-d_{j, k}^{*}\right)^{2}\right) \leq C \sigma_{\epsilon}^{2} / n
$$

$e$

$$
E\left(\left(\hat{d}_{j, k}-d_{j, k}\right)^{2} \mid X_{1}, \ldots, X_{n}\right) \leq C \sigma_{\epsilon}^{2} / n
$$


Se a função distribuição $G$ for conhecida, pode-se representar $f(x)$ como na equação (2.15) e

$$
\tilde{\psi}_{j, k}^{\circ}(x)=\sum_{l: \tilde{g}_{j l k}^{\#} \neq 0} \tilde{g}_{j l k}^{\#} \frac{1_{j+1, l}^{\circ}(x)}{\mu\left(I_{j+1, l}^{\circ}\right)^{1 / 2}}
$$

onde os coeficientes de filtro $\tilde{g}_{j l k}^{\#}$ permitem a $\tilde{\psi}_{j, k}^{\circ}$ ter propriedades similares às de $\tilde{\psi}_{j, k}$ e são entradas de uma matriz de filtro $\tilde{G}_{j}^{\#}$. Também, no modelo (5.6) seja

$$
d_{j, k}^{\circ *}=\int f(x) \tilde{\psi}_{j, k}^{\circ}\left(q_{t}\right)
$$

estimado por

$$
\hat{d}_{j, k}^{\circ *}=n^{-1} \sum_{t=1}^{n} Y_{t}^{*} \tilde{\psi}_{j, k}^{\circ}\left(q_{t}\right)
$$

de modo que se tem a seguinte

Proposição 11 Sob as suposições do modelo (2.10), os coeficientes em (5.16) and (5.17) satisfazem

$$
E\left(\left(\hat{d}_{j, k}^{\circ *}-d_{j, k}^{\circ *}\right)^{2}\right) \leq C \sigma_{\epsilon}^{2} / n
$$

\subsubsection{Estimadores Linear e Não-Linear}

Usando a Proposição 11, um estimador linear

$$
\hat{f}_{\text {lin }}(x)=\sum_{k} \hat{s}_{j_{0}, k}^{\circ} \phi_{j_{0}, k}^{\circ}(x)+\sum_{j=1}^{j_{1}-1} \sum_{k} \hat{d}_{j, k}^{\circ} \psi_{j_{0}, k}^{\circ}(x)
$$

onde $j_{1}=O\left(n^{1 /(1+2 \beta)}\right)$, minimiza o MSE no domínio das ondaletas (DELOUILLE, 2002, Proposição 4.5.6):

$$
\sum_{k} E\left(\hat{s}_{j_{0}, k}^{\circ}-s_{j_{0}, k}^{\circ}\right)^{2}+\sum_{j=1}^{j_{1}-1} \sum_{k} E\left(\hat{d}_{j, k}^{\circ}-d_{j, k}^{\circ}\right)^{2}+\sum_{j=j_{1}}^{\infty} \sum_{k}\left(d_{j, k}^{\circ}\right)^{2} \leq C n^{-\frac{2 \beta}{2 \beta+1}}
$$

Como é difícil estimar $\beta$ e considerando os resultados da Proposição 10, é usualmente sugerido usar o estimador:

$$
\hat{f}(x)=\sum_{k} \hat{s}_{j_{0}, k} \phi_{j_{0}, k}(x)+\sum_{j=1}^{J-1} \sum_{k} \eta_{S}\left(\hat{d}_{j, k}, \lambda_{j, k}\right) \psi_{j_{0}, k}(x),
$$

onde $\eta_{S}(w, \lambda)=\operatorname{sgn}(w)(|w|-\lambda)_{+}, \lambda_{j, k}=\sigma_{j, k} \sqrt{2 \log n}$ e $\sigma_{j, k}=\operatorname{Var}\left(\hat{d}_{j, k}\right)$.

Como o modelo (2.10) assume que os erros são correlacionados, então $\operatorname{Var}\left(\hat{d}_{j, k}\right)=\mathcal{W} \Gamma \mathcal{W}^{T}$, onde $\mathcal{W}$ é a transformada de ondaletas bi-ortogonal em $L_{2}(d \hat{G})$ obtida do esquema de lifting, e $\Gamma$ é a matriz 
de covariância de $\left(\epsilon_{1}, \ldots, \epsilon_{n}\right)$.

Este estimador não precisa de uma estimativa de $\beta$ e, como os erros são gaussianos, ele ainda alcança quase um risco ideal para uma dada amostra $\left\{X_{1}=x_{1}, \ldots, X_{n}=x_{n}\right\}$ (JOHNSTONE; SILVERMAN, 1997, Teorema 1):

$$
\sum_{j} \sum_{k}\left(\eta_{S}\left(\hat{d}_{j, k}, \lambda_{j, k}\right)-\hat{d}_{j, k}\right)^{2} \leq(2 \log n+1)\left(\bar{s}_{n}^{2}+\sum_{j} \sum_{k} \min \left(d_{j, k}^{2}, \sigma_{j, k}^{2}\right)\right),
$$

onde $\bar{s}_{n}^{2}=n^{-1} \sum_{j} \sum_{k} \sigma_{j, k}^{2}$, e a quantidade $\min \left(d_{j, k}^{2}, \sigma_{j, k}^{2}\right)$ é chamada "risco ideal" (DONOHO; JOHNSTONE, 1994).

Um assunto de interesse prático é como estimar $\operatorname{Var}\left(\hat{d}_{j, k}\right)=\mathcal{W} \Gamma \mathcal{W}^{T}$ a partir dos dados. Esperase que o algoritmo de três passos em Delouille (2002, Seção 4.2.4), em geral forneça um estimador razoável. Neste algoritmo, o passo 1 é uma regressão robusta piloto que é usada para se obter os resíduos. No passo 2, estima-se a variância local por outra regressão robusta, usando os resíduos quadráticos. Finalmente, no passo 3, usa-se essa estimativa na aplicação dos limiares.

Uma comparação entre o comportamento dos estimadores linear e não-linear, sob erros i.i.d. e seguindo um modelo $\operatorname{AR}(1)$ com coeficiente $\phi=0,8$, foi realizada para dois sinais: um suave e um com uma descontinuidade. Em ambos os casos a variância do ruído é igual a $(0,1)^{2}$, a variável $X$ de delineamento segue uma distribuição Uniforme $[0,1]$, os sinais foram amostrados em $n=200$ pontos e reconstruídos a partir do nível $j_{0}=2$. As ondaletas duais apresentam 3 momentos nulos enquanto as primais, apenas 1. O estimador linear reconstrói a função até o nível $j=4$ e o suave utiliza limiar duro.

O sinal suave é parte de uma função seno $f(x)=\operatorname{sen}(3 \pi x / 4)+1$, e pode ser observado com os dois tipos de erro na Figura 5.1. Note como a correlação cria tendências locais nos dados. As estimativas lineares e não-lineares, para erros independentes, podem ser obsevadas na Figura 5.2 (a) e (b), respectivamente. Observa-se que o estimador não-linear apresenta um resultado melhor do que o linear. Para erros correlacionados, os resultados estão na Figura 5.2 (c) e (d), respectivamente para os estimadores linear e não-linear. Novamente o estimador não-linear é melhor que o linear, apesar de sofrer influência das tendências locais em torno de $x=0,8$ e $x=0,1$, geradas pelo erro autorregressivo. Observe também que a perda de qualidade do estimador linear é muito maior do que do não-linear, quando se passa de erros independentes para correlacionados.

O mesmo padrão pode ser observado nas Figuras 5.3 e 5.4, para a função salto

$$
\begin{gathered}
f(x)=\left[4 x^{2}(-4 x+3) I(x \leq 0,5)+\left(\frac{4 x}{3}\left(4 x^{2}-10 x+7\right)-\frac{3}{2}\right) I(0,5<x \leq 0,75)\right. \\
\left.+\frac{16 x}{3}(x-1)^{2} I(x>0,75)\right]+1
\end{gathered}
$$



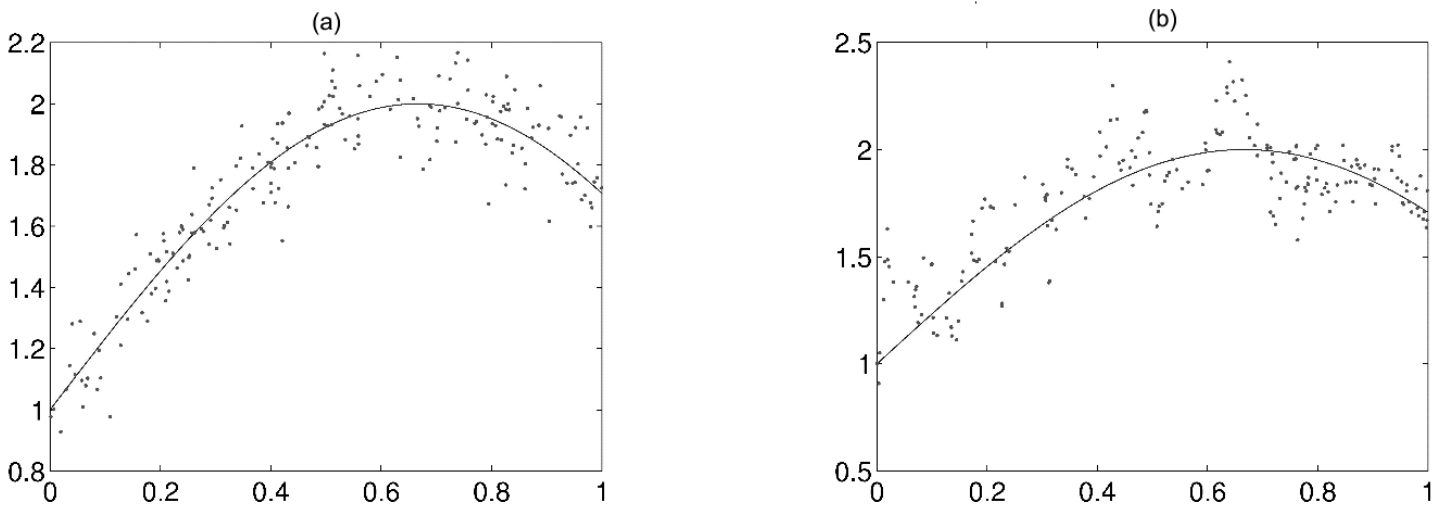

Figura 5.1: (a) Função seno com ruído i.i.d. $N\left(0,(0,1)^{2}\right)$; (b) mesma função de (a), com ruído AR(1), coeficiente $\phi=0,8$ e mesma variância

com uma descontinuidade. Porém, nesse exemplo, apesar do estimador linear não detectar o salto, é o estimador não-linear que sofre mais com as tendências locais geradas pelo ruído autorregressivo.

O aspecto visual do estimador não-linear, na presença de erros correlacionados, em funções com descontinuidades, deixa a desejar supostamente pelo uso de limiar duro, em vez de um suave, e pela ausência do passo de atualização estabilizante. Deste último, principalmente, espera-se uma redução maior nos efeitos gerados pela autocorrelação.

\subsection{Provas da Seção 5.5}

\subsubsection{Prova da Proposição 10}

Não é difícil ver que $E\left(\hat{d}_{j, k}^{*}\right)=d_{j, k}^{*}$ e, portanto o viés é zero. Agora, temos

$$
\begin{aligned}
\operatorname{Var}\left(\hat{d}_{j, k}^{*}\right) & =\operatorname{Var}\left(n^{-1} \sum_{t=1}^{n} Y_{t}^{*} \tilde{\psi}_{j, k}\left(q_{t}\right)\right) \\
& =n^{-2} \operatorname{Cov}\left(\sum_{r=1}^{n} Y_{r}^{*} \tilde{\psi}_{j, k}\left(q_{r}\right), \sum_{s=1}^{n} Y_{s}^{*} \tilde{\psi}_{j, k}\left(q_{s}\right)\right) \\
& =n^{-2} \sigma_{\epsilon}^{2} \sum_{t=1}^{n}\left|\tilde{\psi}_{j, k}\left(q_{t}\right)\right|^{2}+n^{-2} \sum_{r=1}^{n} \sum_{\substack{s=1 \\
s \neq r}}^{n} \tilde{\psi}_{j, k}\left(q_{r}\right) \tilde{\psi}_{j, k}\left(q_{s}\right) \operatorname{Cov}\left(\epsilon_{r}, \epsilon_{s}\right) \\
& \leq n^{-1} \sigma_{\epsilon}^{2} C^{\prime}+n^{-2} \sum_{r=1}^{n} \sum_{\substack{s=1 \\
s \neq r}}^{n}\left|\tilde{\psi}_{j, k}\left(q_{r}\right) \| \tilde{\psi}_{j, k}\left(q_{s}\right)\right|\left|\operatorname{Cov}\left(\epsilon_{r}, \epsilon_{s}\right)\right|
\end{aligned}
$$



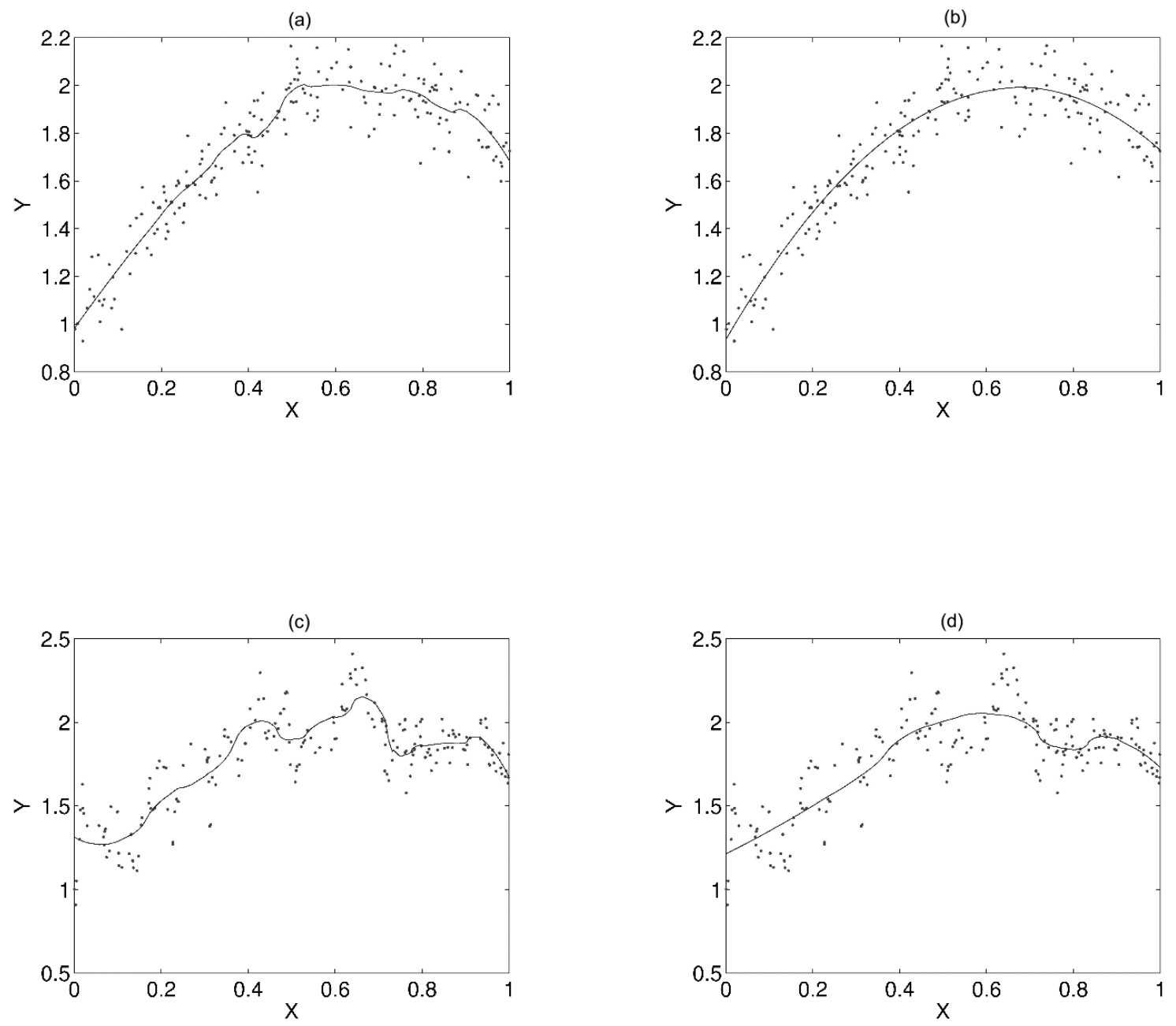

Figura 5.2: Amostras da função seno (pontos) com (a) ruído i.i.d. e estimativa linear ; (b) ruído i.i.d. e estimativa não-linear; (c) ruído $\operatorname{AR}(1)$ com $\phi=0,8$ e estimativa linear; (d) ruído $\operatorname{AR}(1)$ com $\phi=0,8$ e estimativa não-linear. 

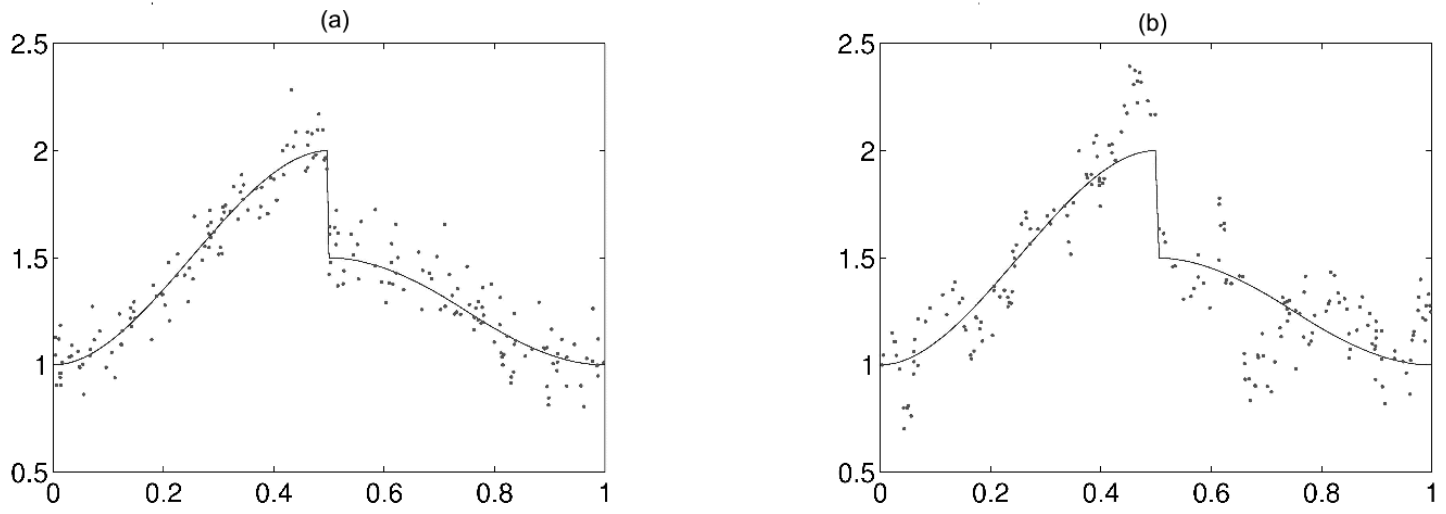

Figura 5.3: (a) Função salto com ruído i.i.d. $N\left(0,(0,1)^{2}\right)$; (b) mesma função de (a), com ruído AR(1), coeficiente $\phi=0,8$ e mesma variância

$$
\leq n^{-1} \sigma_{\epsilon}^{2} C^{\prime}+n^{-2} \sigma_{\epsilon}^{2} \sum_{r=1}^{n} \sum_{\substack{s=1 \\ s \neq r}}^{n}\left|\tilde{\psi}_{j, k}\left(q_{r}\right)\right|\left|\tilde{\psi}_{j, k}\left(q_{s}\right)\right|
$$

pela Proposição 4.5.5, em Delouille (2002) e pela estacionariedade dos erros. Usando a definição em (5.13), o duplo somatório pode ser escrito como

$$
\sum_{l: \tilde{g}_{j l k} \neq 0} \sum_{m: \tilde{g}_{j m k} \neq 0} \sum_{r=1}^{n} \sum_{\substack{s=1 \\ s \neq r}}^{n}\left|\tilde{g}_{j l k}\right|\left|\tilde{g}_{j m k}\right| \frac{1_{j+1, l}^{\circ}\left(q_{r}\right) 1_{j+1, m}^{\circ}\left(q_{s}\right)}{\sqrt{\mu\left(I_{j+1, l}^{\circ}\right)} \sqrt{\mu\left(I_{j+1, m}^{\circ}\right)}}
$$

Mas $1_{j+1, l}^{\circ}\left(q_{r}\right)=1$ se $F_{x}^{-1}(r / n) \in\left[F_{x}^{-1}\left(2^{-(j+1)}(l-1), F_{x}^{-1}\left(2^{-(j+1)} l\right)\right)\right.$. Caso contrário, $1_{j+1, l}^{\circ}\left(q_{r}\right)=$ 0 . Portanto, no somatório acima,

$$
\frac{l-1}{2^{j+1}} \leq r \leq \frac{l}{2^{j+1}}-1, \quad \frac{m-1}{2^{j+1}} \leq s \leq \frac{m}{2^{j+1}}-1,
$$

tal que

$$
\begin{aligned}
\operatorname{Var}\left(\hat{d}_{j, k}^{*}\right) & \leq \frac{\sigma_{\epsilon}^{2} C^{\prime}}{n}+\frac{\sigma_{\epsilon}^{2}}{n^{2}} \sum_{l: \tilde{g}_{j l k} \neq 0} \sum_{m: \tilde{g}_{j m k} \neq 0} \frac{2^{(j+1) / 2}}{2^{j+1}} \frac{2^{(j+1) / 2}}{2^{j+1}}\left|\tilde{g}_{j l k}\right|\left|\tilde{g}_{j m k}\right| \\
& =\frac{\sigma_{\epsilon}^{2} C^{\prime}}{n}+\frac{\sigma_{\epsilon}^{2}}{n^{2} 2^{j+1}} \sum_{l: \tilde{g}_{j l k} \neq 0} \sum_{m: \tilde{g}_{j m k} \neq 0}\left|\tilde{g}_{j l k}\right|\left|\tilde{g}_{j m k}\right| \\
& \leq \frac{\sigma_{\epsilon}^{2} C^{\prime}}{n}+\frac{\sigma_{\epsilon}^{2}}{n^{2} 2^{j+1}} C^{\prime \prime}
\end{aligned}
$$



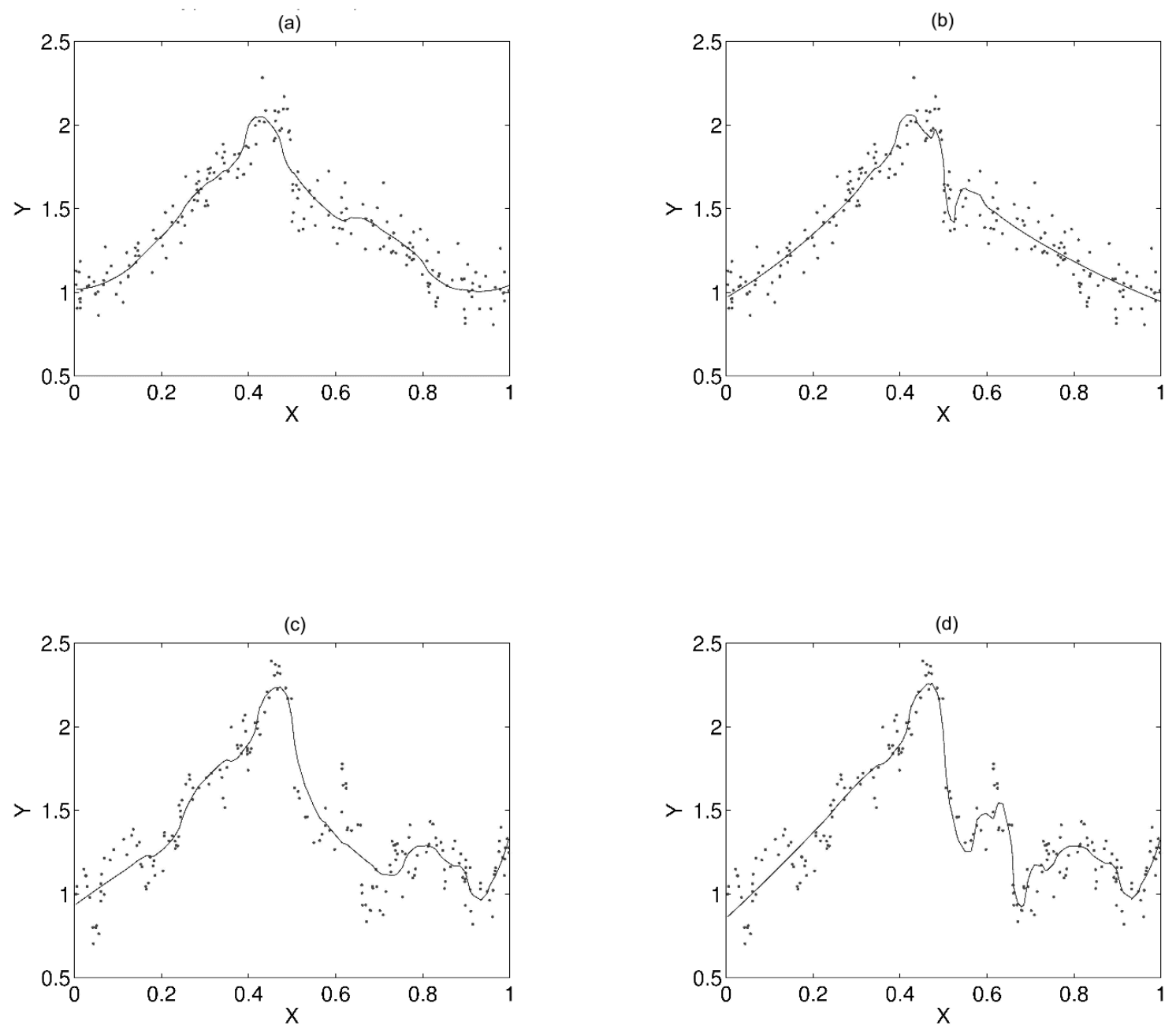

Figura 5.4: Amostras da função salto (pontos) com (a) ruído i.i.d. e estimativa linear ; (b) ruído i.i.d. e estimativa não-linear; (c) ruído $\operatorname{AR}(1) \operatorname{com} \phi=0,8$ e estimativa linear; (d) ruído $\operatorname{AR}(1) \operatorname{com} \phi=0,8$ e estimativa não-linear. 


$$
\leq \frac{\sigma_{\epsilon}^{2} C}{n}
$$

como por construção $\tilde{G}_{j}$ é finito e, pelas suposições sobre $G$, os coeficientes $\tilde{g}_{j l k}$ são uniformemente limitados em $j$.

\subsubsection{Prova da Proposição 11}

Usando (5.15), Proposição 4.5.7 em Delouille (2002) e seguindo os passos iniciais na prova da Proposição 10, pode-se ver que

$$
\operatorname{Var}\left(\hat{d}_{j, k}^{\circ}\right) \leq \frac{\sigma_{\epsilon}^{2}}{n}(C+O(1))+\frac{\sigma_{\epsilon}^{2}}{n^{2} 2^{j+1}} \sum_{l: \tilde{g}_{j l k}^{\#} \neq 0} \sum_{m: \tilde{g}_{j m k}^{\#} \neq 0}\left|\tilde{g}_{j l k}^{\#}\right|\left|\tilde{g}_{j m k}^{\#}\right|,
$$

Como por construção $\tilde{G}_{j}^{\#}$ é finito e, pelas suposições sobre $G$, os coeficientes $\tilde{g}_{j l k}^{\#}$ são uniformemente limitados em $j$,

$$
\operatorname{Var}\left(\hat{d}_{j, k}^{\circ}\right) \leq \frac{\sigma_{\epsilon}^{2}}{n}(C+O(1))+\frac{\sigma_{\epsilon}^{2}}{n^{2} 2^{j+1}} C=\frac{\sigma_{\epsilon}^{2}}{n}(C+O(1)+o(1))
$$

\subsection{Aplicações}

Nesta seção serão analisados dois conjuntos de dados utilizando ondaletas deformadas. O primeiro refere-se à recuperação de créditos inadimplidos, de uma das carteiras de créditos do Banco do Brasil. O segundo foi usado como ilustração em Sardy et al. (1999) e refere-se a observações sobre a magnitude de uma estrela variável.

\subsubsection{Proporção Recuperada de Dívidas}

O processo de recuperação de créditos está contido no de reestruturação de ativos operacionais e envolve diversas estratégias dependentes de muitas variáveis. Como facilitador de algumas etapas desse processo, são requeridas estimativas do valor esperado de recuperação. Mais especificamente, do valor esperado da proporção da dívida que deverá ser recuperada ou reestruturada. Essas estimativas devem ser obtidas para diversos segmentos do mercado, categorias de produtos, tipos de clientes etc. Esta seção será ilustrada com um desses subconjuntos. Este problema foi, dentre outros, um dos motivadores desta tese.

Os dados são uma amostra de 350 dívidas menores do que $R \$ 500.000,00$, de pessoas jurídicas cujas operações de crédido estavam inadimplidas na data da coleta. O objetivo é estimar o valor esperado da proporção da dívida que foi recuperada $R$, quando menor do que 1 , em função do valor da dívida $D$. Como uma das suposições para regressão não-paramétrica usando ondaletas deformadas é de que $D$ tenha distribuição contínua, todas as observações com valores repetidos para $D$ foram substituídas por uma única observação com a mediana dos respectivos valores de $R$. Valores de $R$ maiores ou 


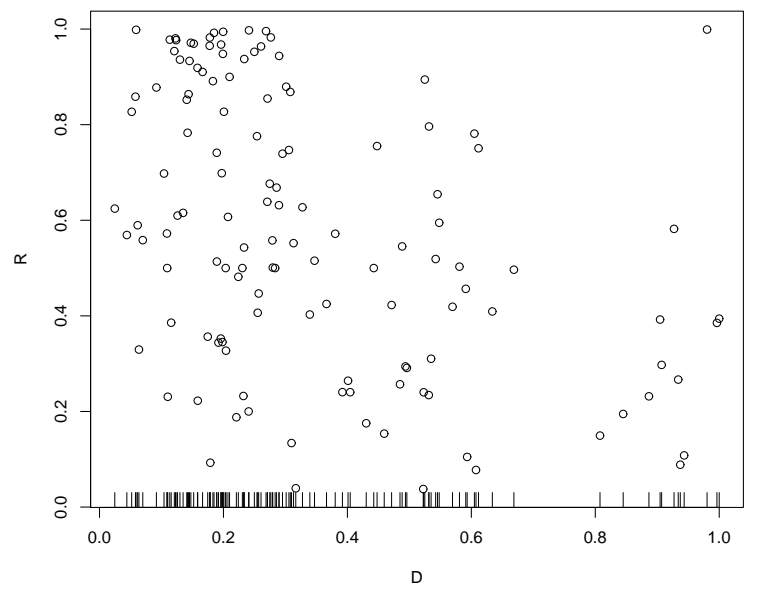

Figura 5.5: Gráfico de dispersão da proporção da dívida que foi recuperada $R$ pelo valor da dívida $D$ e traços logo acima do eixo das abscissas ilustrando o espaçamento desigual de $D$.

iguais a 1 foram retirados da amostra e os valores de $D$ foram transformados para o intervalo $(0,1]$ para preservar a confidência dos dados. Das 164 observações resultantes, uma amostra aleatória de tamanho $2^{7}=128$ foi selecionada sem reposição. O gráfico de dispersão de $R$ por $D$ encontra-se na Figura 5.5, com círculos representando as observações e os traços logo acima do eixo das abscissas ilustrando o espaçamento desigual de $D$. Note também que a distribuição de $D$ é assimétrica à direita, muito diferente de uma Uniforme $[0,1]$.

A experiência prática com esse tipo de dados sugere que a proporção $R$ da dívida que é recuperada, diminui com o aumento do valor $D$ da dívida e, usualmente ajustam-se modelos paramétricos de regressão linear. Isso pode ser observado na Figura 5.5 onde os valores de $R$ são geralmente maiores quando $D<0,4$ do que quando $D>0,8$. A proporção recuperada $R$ também é influenciada por mudanças em políticas, leis e diretrizes internas e externas ao Banco do Brasil. A essas mudanças são atribuídas alterações bruscas e características como a ligeira redução de valores de $R$ próximo a $0,3 \leq D \leq 0,45$. Por sua vez, os valores das dívidas dependem de muitos fatores como tempo desde a obtenção dos créditos, taxa de juros do mercado, tipo e porte da empresa pessoa jurídica etc. Por diversas razões, alguns desses fatores não são usados nos modelos e, usualmente, os resíduos apresentam alguma forma de autocorrelação.

O uso de regressão não-paramétrica via ondaletas, nesses dados, objetiva explorar formas mais complexas da curva de regressão, seguido da identificação, explicação e possível atuação sobre características atípicas e inesperadas. Portanto ajustou-se um modelo de regressão não-paramétrica usando ondaletas deformadas Symmlet 8 e limiares duros por níveis a partir do nível $j=3$. O desvio padrão dos coeficientes de ondaletas foram estimados pelo MAD dos coeficientes de detalhes em cada nível. A estimativa resultante pode ser observada na Figura 5.6 (a). Há indicativo de presença de autocorrelação nos dados (Figura 5.6 (b)), pois a variância dos coeficientes de ondaletas diminui quando o 
(a)

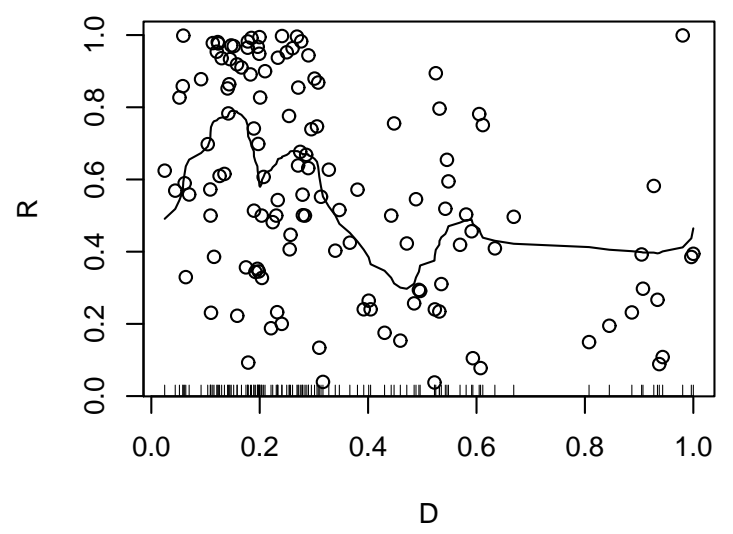

(c)

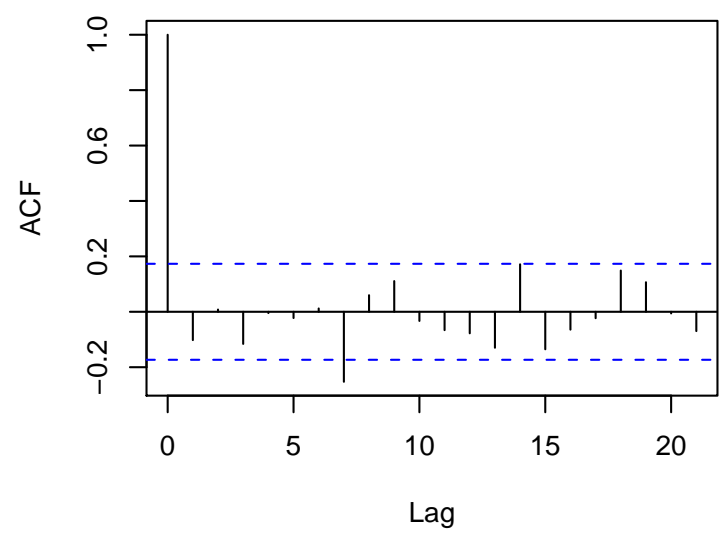

(b)

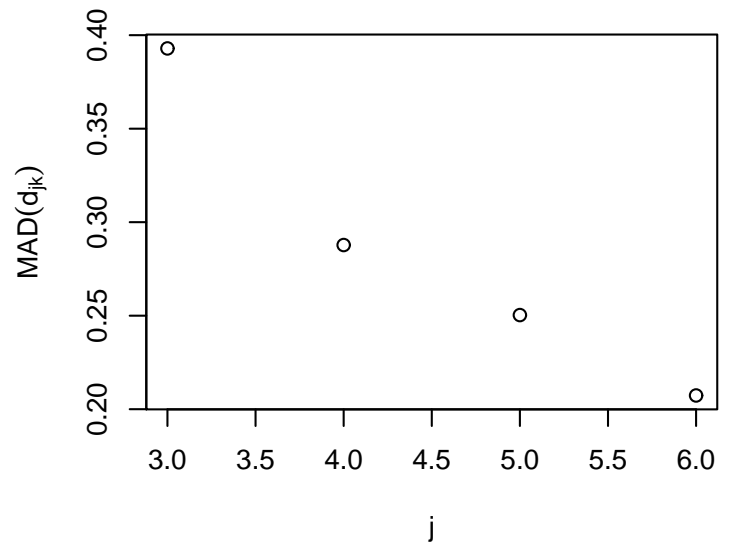

(d)

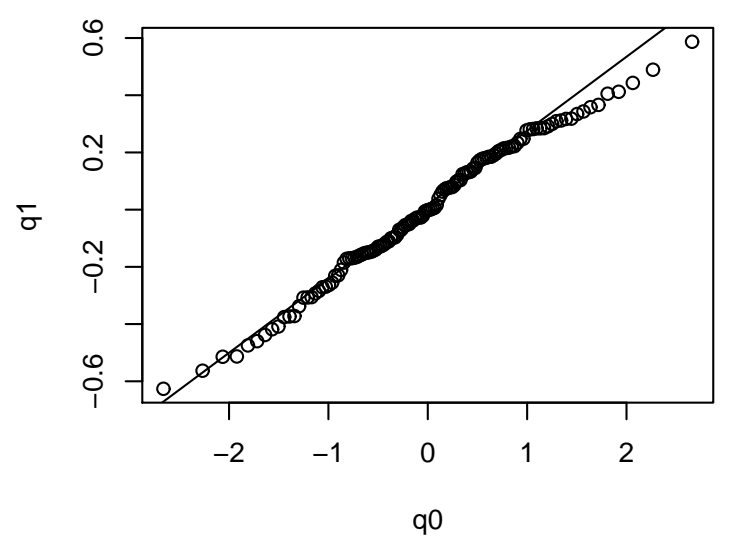

Figura 5.6: (a) Gráfico de dispersão da proporção da dívida que foi recuperada $R$ pelo valor da dívida $D$ (círculos), traços sobre o eixo das ordenadas ilustrando o espaçamento desigual de $D$ e estimativa usando ondaletas deformadas (linha) com limiares por níveis; (b) verificação de autocorrelação; (c) correlograma dos resíduos; (d) gráfico de quantis normais teóricos $q_{0}$ e amostrais $q_{1}$ e reta passando pelos primeiro e terceiro quantis.

nível de resolução aumenta (JOHnSTONE; SILVERMan, 1997). Porém, pelos resíduos (Figura 5.6 (c)), aparentemente a autocorrelação é estacionária e fraca. Finalmente, o gráfico de quantis na Figura 5.6 (d) não indica grandes desvios da hipótese de normalidade. Portanto, o uso de ondaletas deformadas parece apropriado.

Pela estimativa constante na Figura 5.6 (a), pode-se observar as características citadas: redução em $R$ à medida em que $D$ aumenta; redução de $R$ para $0,3 \leq D \leq 0,5$, seguido de um aumento para $D>0,5$. Entretanto, três outras características não antecipadas foram detectadas: há uma queda brusca na recuperação $R$ próximo a $D=0,2$; a recuperação cresce entre aproximadamente $0<D \leq 1,5$; há uma ligeira queda em $D=0,6$, seguida de uma redução suave, diferentemente 
(a)

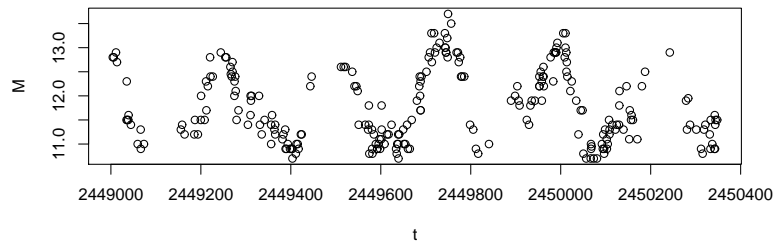

(b)

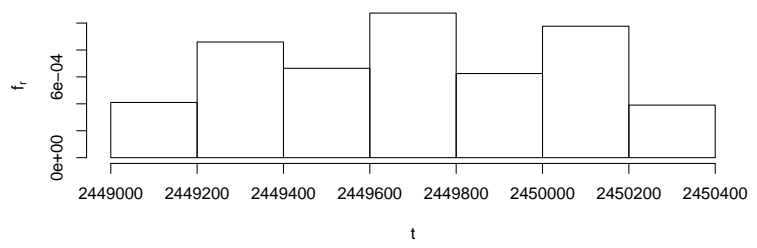

Figura 5.7: (a) Gráfico de dispersão de 256 magnitudes $M$ da cefeida RU Andrômeda pelos dias julianos $t$ entre 2449004 e 2450352 (Janeiro de 1993 a meados de 1996). (b) Histograma da distribuição de 256 dias $t$ de observação da cefeida.

do padrão de queda para $D<0,6$. Essas três especificidades podem indicar falhas no processo, oportunidades de negociação, alterações na legislação etc. que poderão ser investigadas. O destaque, entretanto, é para o fato de não terem sido notadas de imediato e de serem ignoradas por modelos lineares paramétricos.

\subsubsection{Magnitude de Cefeidas}

Como outro exemplo de conjunto de dados com delineamento desigualmente espaçado, considere o problema de se estimar a curva de luz da estrela variável (cefeida) RU Andrômeda. As magnitudes $M$ dessa estrela são medidas visualmente, em datas $t$ irregularmente espaçadas devido ao bloqueio da visão pela luz do sol, condições atmosféricas e disponibilidade de tempo de uso dos telescópios.

Os dados foram obtidos da Base de Dados Internacional da Associação Americana de Observadores de Estrelas Variáveis (AAVSO), mantida por J. A. Mattei e publicamente disponível na Internet em http://www aavso org. Os dados baixados em 16 de outubro de 2007 consistiam de 301 observações, referentes ao período compreendido entre os dias julianos $t_{a}=2449004$ e $t_{b}=2450352$ (Janeiro de 1993 a meados de 1996). Como no exemplo anterior, todas as observações com datas $t$ repetidas foram substituídas por uma única observação com a mediana das magnitudes $M$. Das 292 observações resultantes, uma amostra aleatória de tamanho $2^{8}=256$ foi selecionada sem reposição. O gráfico de dispersão de $M$ por $t$ encontra-se na Figura 5.7 (a). Um histograma da distribuição de $t$ encontra-se na Figura 5.7 (b). O teste de Kolmogorov-Smirnov rejeita a hipótese de uma distribuição Uniforme $\left[t_{a}, t_{b}\right]$ para os dias, a qualquer nível de significância ( $\mathrm{p}$-valor $<2,2 \cdot 10^{-16}$ ).

Ajustou-se um modelo de regressão não-paramétrica usando ondaletas deformadas Symmlet 8 e limiares duros por níveis a partir do nível $j=4$. O desvio padrão dos coeficientes de ondaletas 
(a)

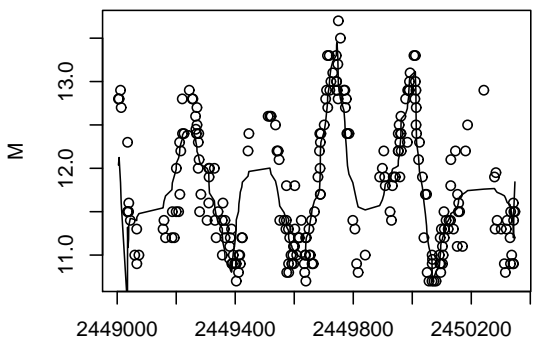

(c)

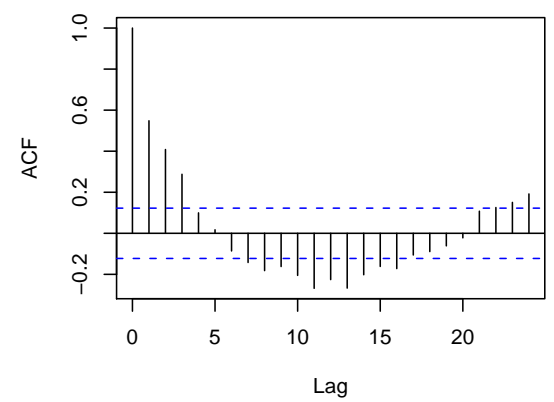

(b)

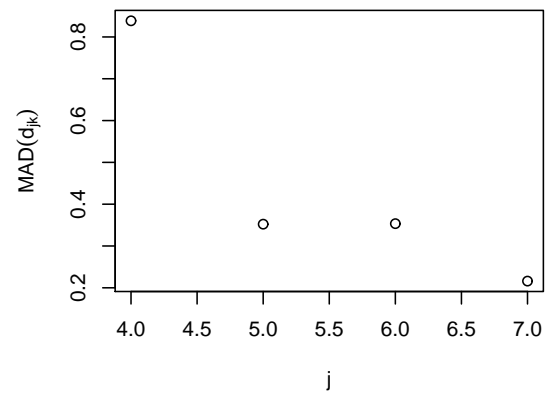

(d)

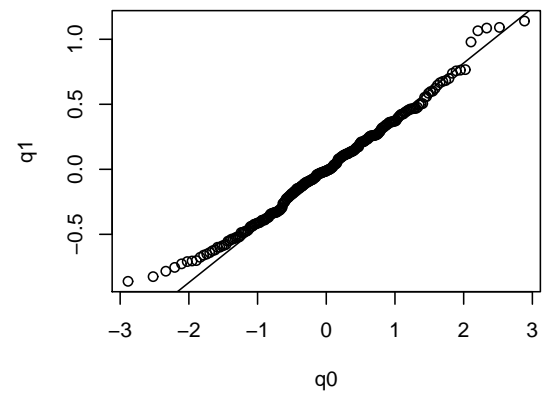

Figura 5.8: (a) Gráfico de dispersão de 256 magnitudes $M$ da cefeida RU Andrômeda pelos dias julianos $t$ entre 2449004 e 2450352 (círculos) e estimativa usando ondaletas deformadas (linha) com limiares por níveis; (b) verificação de autocorrelação; (c) correlograma dos resíduos; (d) gráfico de quantis normais teóricos $q_{0}$ e amostrais $q_{1}$ e reta passando pelos primeiro e terceiro quantis.

foram estimados pelo MAD dos coeficientes de detalhes em cada nível. A estimativa resultante pode ser observada na Figura 5.8 (a). Há indicativo de presença de autocorrelação nos dados (Figura 5.8 (b)), pois a variância dos coeficientes de ondaletas diminui quando o nível de resolução aumenta (JOHnStONE; SILVERMAN, 1997). Pelos resíduos (Figura 5.8 (c)), aparentemente a autocorrelação é estacionária mas não desprezível. Finalmente, o gráfico de quantis na Figura 5.8 (d) não indica grandes desvios da hipótese de normalidade, apesar de sugerir caudas mais pesadas do que as da distribuição normal. Portanto, o uso de ondaletas deformadas parece apropriado.

Comparando-se com o mesmo estimador, porém aplicando o limiar Universal (DONOHO; JOHNSTONE, 1994, 1995), verifica-se a possível influência da autocorrelação ao elevar os dois primeiros pontos de máximo (Figura 5.9 (a)). Pela Figura 5.9 (b), nota-se que a correlação dos resíduos foi eliminada e absorvida pela estimativa. 
(a)

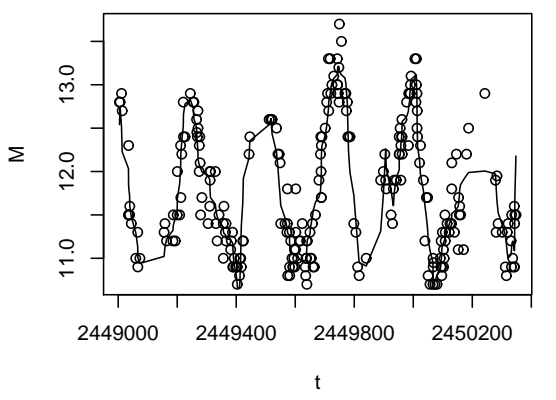

(c)

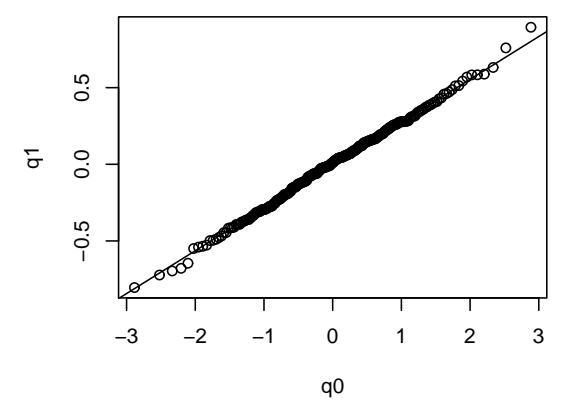

(b)

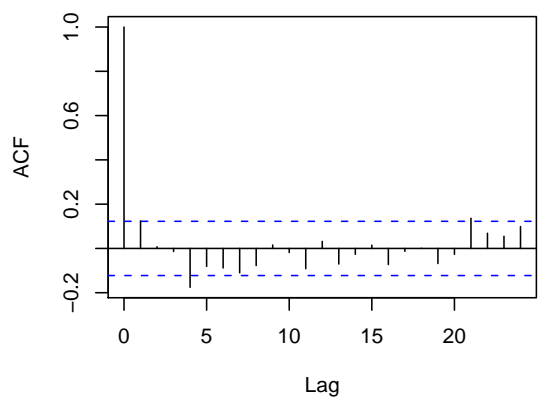

Figura 5.9: (a) Gráfico de dispersão de 256 magnitudes $M$ da cefeida RU Andrômeda pelos dias julianos $t$ entre 2449004 e 2450352 (cículos) e estimativa usando ondaletas deformadas (linha) com limiar Universal; (b) correlograma dos resíduos; (c) gráfico de quantis normais teóricos $q_{0}$ e amostrais $q_{1}$ e reta passando pelos primeiro e terceiro quantis. 


\section{Capítulo 6}

\section{Procedimento Semi-paramétrico}

Neste capítulo, apresenta-se uma abordagem alternativa ao procedimento de suavização via ondaletas, para um modelo de séries temporais composto por um sinal adicionado a erros autorregressivos estacionários. O objetivo é estimar o sinal globalmente, com risco próximo ao mínimo possível. A abordagem usual a esse problema é aplicar limiares aos coeficientes de ondaletas, com diferentes limiares em cada nível de resolução. Neste capítulo, a autocorrelação é tratada de modo paramétrico, permitindo que os métodos de ondaletas sejam usados apenas para a estimação da função. Portanto, é proposto um método iterativo semi-paramétrico. Este método iterativo toma emprestado algumas ideias do procedimento de Cochrane e Orcutt (1949). Os resultados de simulação mostram que o método proposto é pelo menos tão bom quanto os melhores métodos usados com ondaletas, independentemente do tipo de autocorrelação presente nos erros.

\subsection{Modelo e Suposições}

Seja $y_{1}, y_{2}, \ldots, y_{n}$ uma sequência de observações do modelo

$$
Y_{t}=f(t / n)+\epsilon_{t}
$$

onde $t=1,2, \ldots, n, Y_{t}$ é uma variável aleatória, $f$ é uma função desconhecida a ser estimada e a sequencia $\left\{\epsilon_{t}\right\}_{t=1}^{n}$ é gerada por um processo Gaussiano de média zero. Este processo pode ser autocorrelacionado ou não.

O objetivo é estimar $f$ globalmente. Uma medida do desempenho de um estimador $\hat{f}$ é o risco global obtido pelo valor esperado do quadrado da norma $L_{2}$ :

$$
R(\hat{f}, f)=E\left(\int_{0}^{1}(\hat{f}(t)-f(t))^{2} d t\right)
$$

onde a integral deve estar bem definida. 


\subsection{Procedimento}

Nesta Seção, é descrito o procedimento proposto, de um modo intuitivo e são dadas algumas justificativas para seu desempenho.

O estimador proposto é obtido como um resultado de um processo iterativo que toma emprestado ideias do procedimento de Cochrane e Orcutt (1949). A útil propriedade (2.6) da política de aplicação de limiares, é também similar ao que é obtido a partir do método dos mínimos quadrados. Regressão com erros em séries temporais, usando o tempo como variável explicativa e mínimos quadrados ordinários, leva a estimadores não-viesados e consistentes quando a série temporal é estacionária, uma vez que eles geralmente satisfazem as condições de Grenander (FUller, 1996). Como os estimadores de mínimos quadrados, o uso de limiares aplicados aos coeficientes de ondaletas, ignorando a correlação dos erros, fornece estimadores não-ótimos, em geral. Portanto, alguma melhoria pode ser alcançada através da aplicação de limiares por níveis.

Em vez de usar algoritmos que aplicam limiares por níveis, o método proposto é aplicar um simples limiar aos coeficientes de ondaletas e estimar a matriz de covariância dos erros a partir dos resíduos resultantes. Este processo seria iterado até que um critério de convergência fosse alcançado.

Suponha que se postule o modelo (6.1) e se use o estimador (2.5) como um estimador primário. Então, são obtidos os resíduos

$$
e_{t}=y_{t}-\hat{f}(t / n), \quad t=1, \ldots, n \text {. }
$$

Como $\hat{f}$ é um estimador consistente da verdadeira função $f$, então $(R(\hat{f}, f) \stackrel{n \rightarrow \infty}{\longrightarrow} 0)$, e os resíduos $e_{t}$ são bons preditores dos erros $\epsilon_{t}, t=1, \ldots, n$. Como a sequência $\left\{\epsilon_{t}\right\}_{1}^{n}$ é oriunda de um processo estacionário Gaussiano de média zero, ela pode ser modelada como um processo $\operatorname{AR}(p)$, com $p$ suficientemente grande. Isto pode ser realizado de um modelo automático usando algum critério de informação como o de Akaike (AIC) ou o BIC de Schwarz. Propõe-se escolher $p$ como a ordem do processo que minimiza o $\operatorname{AIC}$ do modelo $\operatorname{AR}(p)$ ajustado à sequência de resíduos $\left\{e_{t}\right\}_{1}^{n}$. Denote este modelo por

$$
e_{t}=\mu+\phi_{1} e_{t-1}+\ldots+\phi_{p} e_{t-p}+a_{t},
$$

onde os $a_{t}$ são variáveis aleatórias i.i.d. $N\left(0, \sigma_{\epsilon}^{2}\right)$, for $t=1, \ldots, n$.

As estimativas de $\phi_{1}, \ldots, \phi_{p}$ em (6.2) podem ser usadas para construir uma estimativa da matriz de covariância $\Gamma_{n}$ dos erros com uma estrutura $\operatorname{AR}(p)$. Sendo $\left\{e_{t}\right\}_{1}^{n}$ um processo estacionário real de média zero e função de autocovariância $\gamma_{\tau}=E\left(e_{t} e_{t+\tau}\right)$, então se $e_{t}$ seguir um processo $\operatorname{AR}(p)$, pode-se mostrar (MORETTIN, 1999) que para $\tau \geq 0$,

$$
\gamma_{0}=\frac{\sigma_{\epsilon}^{2}}{1-\phi_{1} \rho_{1}-\ldots-\phi_{p} \rho_{p}}, \quad \text { para } \tau=0,
$$


$\mathrm{e}$

$$
\gamma_{\tau}=\phi_{1} \gamma_{\tau-1}+\phi_{2} \gamma_{\tau-2}+\ldots+\phi_{p} \gamma_{\tau-p}, \quad \text { para } \tau>0
$$

A ideia é que as estimativas $\hat{\gamma}_{\tau} \approx r_{|s-t|}$ para $\tau=|s-t|$, onde $r_{|s-t|}$ são as entradas do matriz de covariância $\Gamma_{n}$ que geram uma matriz de covariâncias estimada $\hat{\Gamma}_{n}$.

Uma vez que se obtém $\hat{\Gamma}_{n}$, se está pronto para decorrelacionar os dados de modo a obter um eficiente estimador suavisado da função $f$, baseado em ondaletas. Denote por

$$
\hat{\Gamma}_{n}=\hat{S}_{n} \hat{S}_{n}^{\prime}
$$

a decomposição de Cholesky da matriz de covariâncias estimada, uma vez que ela deve ser positiva definida. Para decorrelacionar os erros originais, simplesmente faça

$$
Y^{\prime}=\hat{S}_{n}^{-1} Y
$$

e, então, obtenha um estimador eficiente de ondaletas através de limiarização simples, $\hat{f}^{\prime}$, digamos.

O passo final neste procedimento é aplicar de volta $\hat{S}_{n}$ a $\hat{f}^{\prime}$ devolvendo a estrutura de covariância que foi estimada de modo a se obter uma nova estimativa para a verdadeira função $f$. Chame esta estimativa de $\hat{f}^{(1)}$.

Esse processo pode ser iterado produzindo estimativas $\hat{f}^{(2)}, \ldots, \hat{f}^{(s)}, \ldots$ até que seja alcançado um critério de convergência previamente selecionado. Como o principal objetivo de métodos de limiarização de coeficientes de ondaletas é a minimização do risco $R(\hat{f}, f)$, uma agradável escolha é minimizar o erro médio quadrático (MSE) definido por

$$
M S E(s)=\frac{1}{n} \sum_{t=1}^{n}\left(\hat{f}^{(s)}(t / n)-Y_{t}\right)^{2}
$$

onde $\hat{f}^{(s)}(t / n)$ é o estimador obtido da $s$-ésima iteração através da aplicação de uma tranformada discreta inversa de ondaletas. O processo iterativo continua até $\min _{s} M S E\left(\hat{f}^{(s)}, Y\right)$ ser alcançado. Simulações mostram que a convergência é alcançada em poucas iterações, usualmente 4 ou 5 , e o processo inteiro é muito rápido mesmo para grandes valores de $n$, e.g., $n=2048$.

Brevemente, os passos sugeridos são:

1. postule o modelo (2.10) e use o estimador $(2.5), \hat{f}^{(0)}=W^{T} \hat{\theta}$, como um estimador inicial e calcule $M S E(0)$;

2. calcule os resíduos $e_{t}=y_{t}-\hat{f}^{(0)}(t / n), \quad t=1, \ldots, n$;

3. ajuste um modelo autorregressivo $\operatorname{AR}(p)$ aos resíduos escolhendo a ordem $p$ que minimiza o AIC e faça $s=0$; 
4. use as estimativas de $\phi_{1}, \ldots, \phi_{p}$ do modelo ajustado para construir uma estimativa da matriz de covariância $\Gamma_{n}$ através da equação em diferenças (6.4);

5. encontre a decomposição de Cholesky da matriz de covariância estimada dos erros denotada por $\hat{S}_{n}$, e decorrelacione os resíduos fazendo $Y^{\prime}=\hat{S}_{n}^{-1} Y$;

6. aplique um algoritmo de limiarização de ondaletas aos dados decorrelacionados $Y^{\prime}$ de modo a obter um estimador $\hat{f}^{\prime}$;

7. calcule os resíduos $e_{i}^{(s)}=y_{i}-\hat{f}^{(s)}(i / n), \quad i=1, \ldots, n$;

8. faça $s=s+1$; multiplique $\hat{S}_{n}$ a $\hat{f}^{\prime}$ e obtenha uma nova estimativa $\hat{f}^{(s)}$ para a verdadeira função $f$

9. calcule $M S E(s)$ como em (6.5):

9a. se $M S E(s-1)-M S E(s)$, para $s>0$, for maior do que o critério de convergência, volte ao passo 4 ;

9b. se $M S E(s-1)-M S E(s)$, para $s>0$, for menor ou igual ao critério de convergência, pare.

Um ponto importante é que a ordem do processo $\operatorname{AR}(p)$ deve ser escolhida apenas uma vez no início do processo iterativo, de modo a alcançar convergência do algoritmo.

\subsection{Simulações}

De modo a avaliar o desempenho do método iterativo proposto, algumas simulações foram feitas justamente com outros dois procedimentos concorrentes, usando limiar Universal (DONOHO; JOHNSTONE, 1994, 1995) e SURE por níveis (JOHNSTONE; SILVERMAN, 1997). A conservadora propriedade (2.6) do limiar Universal vêm junto com um preço: em termos de perda $L^{2}$, melhor desempenho poderia ser obtido com limiares menores, alcançados pela escolha de uma política SURE por níveis (JOHNSTONE; SILVERMAN, 1997), no caso de erros correlacionados. Uma escolha de política de limiar SURE baseada nos dados pode então ser obtida simplesmente minimizando-se um estimador não-viesado do erro médio quadrático com respeito a um possível limiar, no intervalo $[0, \sigma \sqrt{2 \log n}$.

A simulação foi feita com 3 diferentes funções. Para cada função $f(t / n)$ foi adicionado um ruído que poderia ser um ruído branco Gaussiano, ou um ruído autorregressivo de ordem 1 com coeficiente $\phi_{1}=0,8$ ou $\phi_{1}=-0,8$. Cada uma dessas nove simulações foi feita com 3 tamanhos de amostra $(n=$ 64,128 e 256 pontos) e replicada 1000 vezes. Os erros foram re-escalonados para que as simulações fossem feitas com $S N R=1$, onde

$$
S N R=\frac{(n-1)^{-1} \sum_{t}(f(t / n)-\bar{f})^{2}}{\operatorname{Var}(\text { ruído })}
$$


(a)

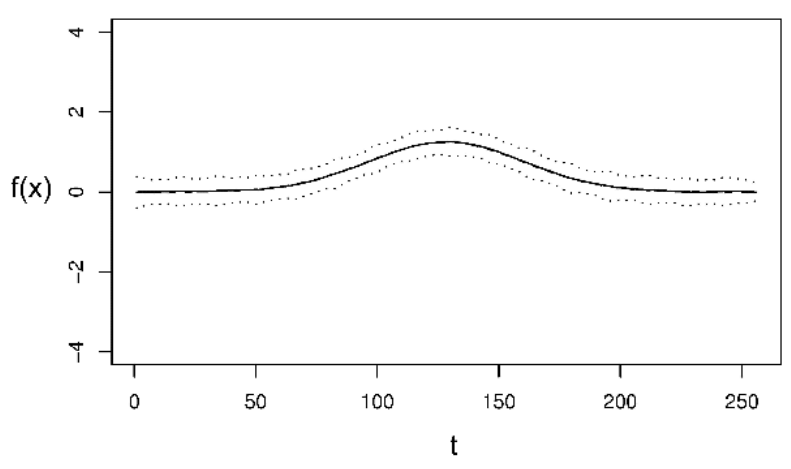

(c)

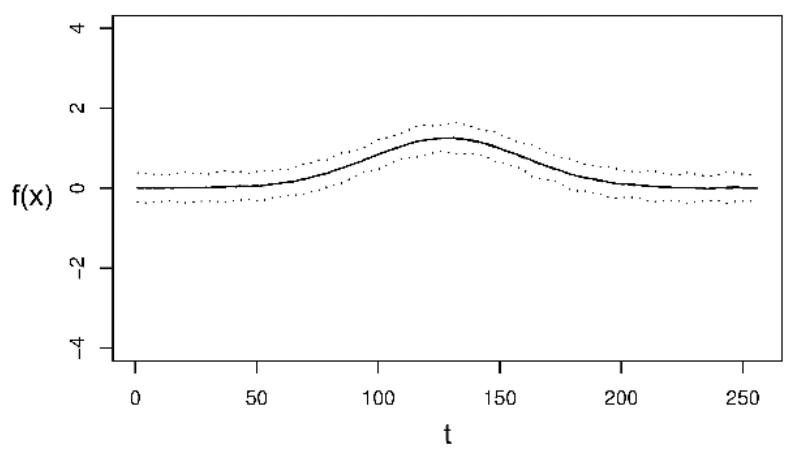

(b)

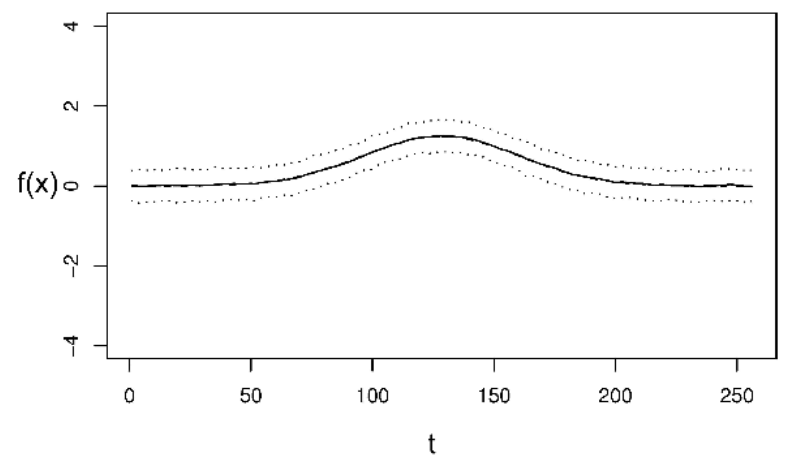

Figura 6.1: Resultados de simulação para a função densidade Gaussiana, coeficiente autorregressivo igual a 0,8, tamanho da amostra igual a 256; estimador (linha contínua), função verdadeira (linha tracejada) e estimador \pm 1 erro padrão (linha pontilhada). Métodos (a) iterativo, (b) Universal e (c) SURE.

e $\bar{f}=n^{-1} \sum_{t} f(t / n)$

As funções usadas nas simulações foram, para $t=1, \ldots, n$ :

- densidade Gaussiana: $f(t / n)=100 \times(n / 8(\phi(t)+n / 2))$, onde $\phi(\cdot)$ denota a densidade Normal padrão;

- função HeaviSine: $f(t / n)=4 \operatorname{sen}(4 \pi t / n)-\operatorname{sgn}(t / n-0,3)-\operatorname{sgn}(0,72-t / n)$;

- função Doppler: $f(t / n)=\sqrt{(1-t / n) t / n} * \operatorname{sen}(2 \pi(1+0,05) /(t / n+0,05))$.

Estas funções são mostradas em linha tracejada nas Figuras 6.1, 6.2 e 6.3, respectivamente.

Ao final, algumas estatísticas foram calculadas que permitem uma útil comparação e análise. As estatísticas computadas foram as versões amostrais do viés quadrático, média aritmética do MSE (6.5) e erro padrão do MSE. Comentários sobre os cálculos realizados são apresentados na próxima 
(a)

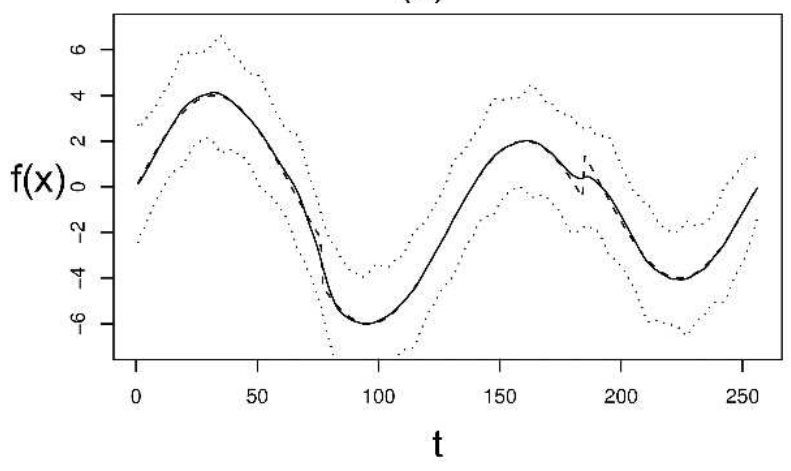

(c)

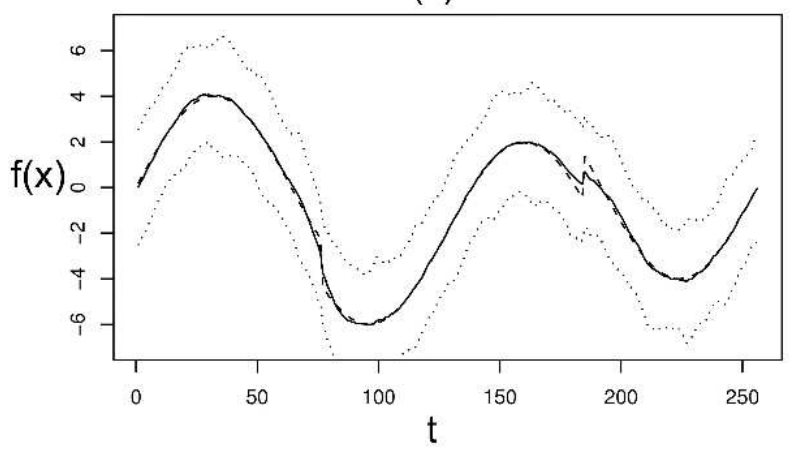

(b)

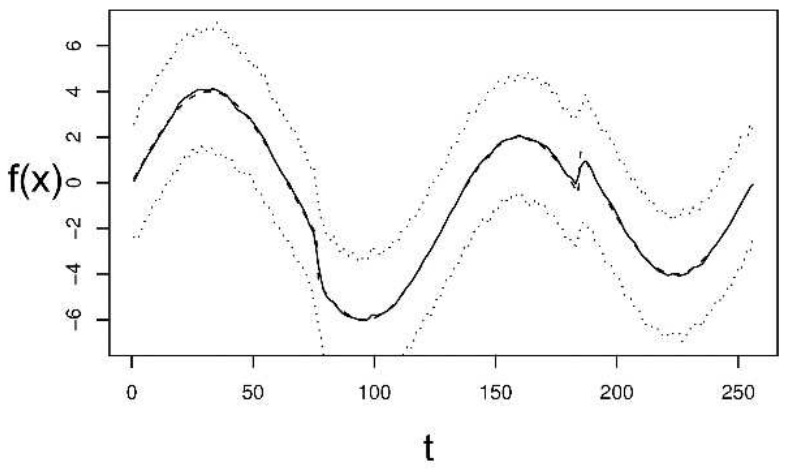

Figura 6.2: Resultados de simulação para a função HeaviSine, coeficiente autorregressivo igual a 0,8, tamanho da amostra igual a 256; estimador (linha contínua), função verdadeira (linha tracejada) e estimador \pm 1 erro padrão (linha pontilhada). Métodos (a) iterativo, (b) Universal e (c) SURE.

Seção. Todas as simulações foram feitas usando a liguagem R (R Development Core Team, 2005). Os cálculos de ondaletas foram feitas em R com o pacote Waveslim (WHITCHER, 2005).

\subsubsection{Resultados}

Nesta Seção os resultados das simulações são discutidos. Os métodos de estimação serão denominados simplesmente como "Universal", "SURE" e "iterativo", em vez de "estimador de ondaletas usando o limiar Universal", por exemplo.

Figura 6.1 e Tabela 1 mostram os resultados para o caso Gaussiano. Visualmente, todos os três métodos apresentam resultados equivalentes em termos de média e variabilidade. Para tamanhos menores de amostra, o método iterativo parece apresentar menos variabilidade e menos viés quando os erros são correlacionados. Para erros não-correlacionados, os estimadores SURE parecem ter maior média de MSE. 
(a)

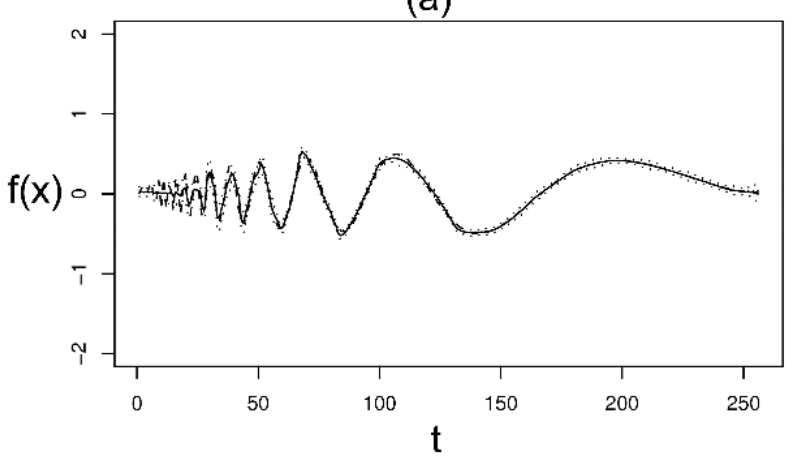

(c)

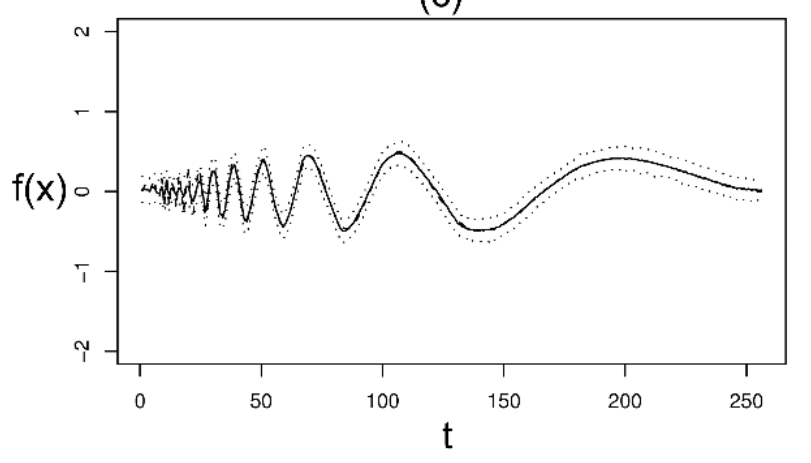

(b)

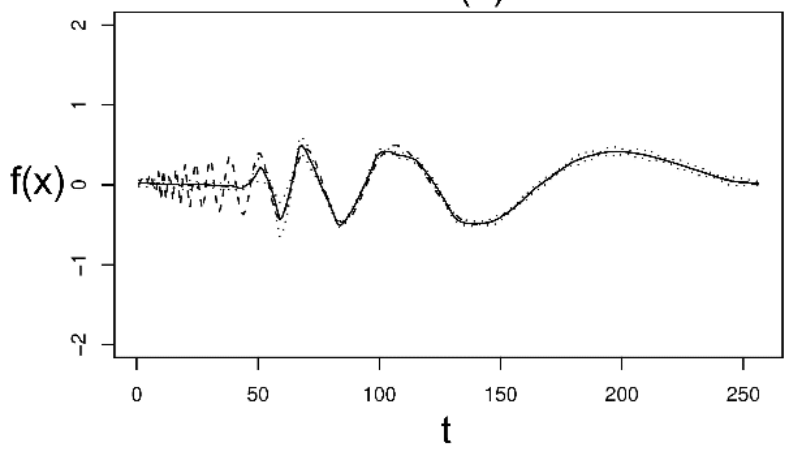

Figura 6.3: Resultados de simulação para a função Doppler, coeficiente autorregressivo igual a 0,8, tamanho da amostra igual a 256; estimador (linha contínua), função verdadeira (linha tracejada) e estimador \pm 1 erro padrão (linha pontilhada). Métodos (a) iterativo, (b) Universal e (c) SURE.

Tabela 6.1: Simulação de função Gaussiana.

\begin{tabular}{cc|ccc|ccc|ccc}
\hline & & \multicolumn{3}{|c|}{ Iterativo } & \multicolumn{3}{c|}{ Universal } & \multicolumn{3}{c}{ SURE por nível } \\
\hline $\mathrm{N}$ & AR & Vies $^{2}$ & MSE & e.p.(MSE) & Vies $^{2}$ & MSE & e.p.(MSE) & Vies $^{2}$ & MSE & e.p.(MSE) \\
\hline \multirow{2}{*}{64} & 0 & 0,218 & 0,728 & 0,011 & 0,218 & 0,739 & 0,011 & 0,249 & 1,434 & 0,021 \\
& 0,8 & 0,018 & 2,544 & 0,030 & 0,006 & 2,898 & 0,025 & 0,251 & 2,376 & 0,030 \\
& $-0,8$ & 0,032 & 0,323 & 0,014 & 0,352 & 0,503 & 0,010 & 0,324 & 1,253 & 0,031 \\
\hline \multirow{2}{*}{128} & 0 & 0,002 & 0,082 & 0,002 & 0,002 & 0,078 & 0,002 & 0,001 & 0,292 & 0,005 \\
& 0,8 & 0,001 & 0,495 & 0,005 & $<0,001$ & 0,650 & 0,003 & 0,001 & 0,503 & 0,005 \\
& $-0,8$ & 0,002 & 0,031 & 0,002 & 0,002 & 0,026 & 0,002 & 0,001 & 0,217 & 0,007 \\
\hline \multirow{2}{*}{256} & 0 & $<0,001$ & 0,016 & $<0,001$ & $<0,001$ & 0,015 & $<0,001$ & $<0,001$ & 0,064 & 0,001 \\
& 0,8 & $<0,001$ & 0,104 & 0,001 & $<0,001$ & 0,155 & $<0,001$ & $<0,001$ & 0,123 & 0,001 \\
& $-0,8$ & $<0,001$ & 0,005 & $<0,001$ & $<0,001$ & 0,004 & $<0,001$ & $<0,001$ & 0,048 & 0,002 \\
\hline
\end{tabular}


Tabela 6.2: Simulação de função HeaviSine.

\begin{tabular}{cc|ccc|ccc|ccc}
\hline & & \multicolumn{3}{|c|}{ Iterativo } & \multicolumn{3}{c|}{ Universal } & \multicolumn{3}{c}{ SURE por nível } \\
\hline $\mathrm{N}$ & AR & Vies $^{2}$ & MSE & e.p.(MSE) & Vies $^{2}$ & MSE & e.p.(MSE) & Vies $^{2}$ & MSE & e.p.(MSE) \\
\hline \multirow{2}{*}{64} & 0 & 1,556 & 3,824 & 0,033 & 1,138 & 3,548 & 0,030 & 2,779 & 6,125 & 0,060 \\
& 0,8 & 0,220 & 8,833 & 0,083 & 0,045 & 8,640 & 0,077 & 2,281 & 8,565 & 0,088 \\
& $-0,8$ & 0,271 & 1,486 & 0,049 & 2,958 & 3,414 & 0,027 & 2,924 & 5,433 & 0,088 \\
\hline \multirow{2}{*}{128} & 0 & 0,072 & 5,742 & 0,057 & 0,025 & 7,534 & 0,037 & 0,126 & 5,931 & 0,052 \\
& 0,8 & 0,129 & 0,574 & 0,019 & 0,266 & 0,557 & 0,020 & 0,089 & 2,657 & 0,084 \\
& $-0,8$ & 0,040 & 0,263 & 0,010 & 0,048 & 0,227 & 0,010 & 0,016 & 2,241 & 0,075 \\
\hline \multirow{2}{*}{256} & 0 & 0,045 & 0,780 & 0,011 & 0,045 & 0,736 & 0,010 & 0,021 & 2,937 & 0,047 \\
& 0,8 & 0,034 & 4,915 & 0,037 & 0,015 & 7,178 & 0,021 & 0,025 & 5,775 & 0,038 \\
& $-0,8$ & 0,040 & 0,263 & 0,010 & 0,048 & 0,227 & 0,010 & 0,016 & 2,241 & 0,075 \\
\hline
\end{tabular}

Tabela 6.3: Simulação de função Doppler.

\begin{tabular}{cc|ccc|ccc|ccc}
\hline & & \multicolumn{3}{|c|}{ Iterativo } & \multicolumn{3}{c|}{ Universal } & \multicolumn{3}{c}{ SURE por nível } \\
\hline $\mathrm{N}$ & AR & Vies $^{2}$ & MSE & e.p.(MSE) & Vies $^{2}$ & MSE & e.p.(MSE) & Vies $^{2}$ & MSE & e.p.(MSE) \\
\hline \multirow{2}{*}{64} & 0 & 0,027 & 0,054 & $<0,001$ & 0,023 & 0,051 & $<0,001$ & 0,026 & 0,068 & 0,001 \\
& 0,8 & 0,005 & 0,088 & 0,001 & 0,004 & 0,089 & 0,001 & 0,020 & 0,090 & 0,001 \\
& $-0,8$ & 0,013 & 0,036 & 0,001 & 0,047 & 0,055 & $<0,001$ & 0,027 & 0,056 & 0,001 \\
\hline \multirow{3}{*}{128} & 0 & 0,013 & 0,027 & $<0,001$ & 0,013 & 0,026 & $<0,001$ & 0,005 & 0,043 & $<0,001$ \\
& 0,8 & 0,005 & 0,070 & $<0,001$ & 0,002 & 0,078 & $<0,001$ & 0,004 & 0,070 & $<0,001$ \\
& $-0,8$ & 0,007 & 0,014 & $<0,001$ & 0,017 & 0,022 & $<0,001$ & 0,003 & 0,030 & 0,001 \\
\hline \multirow{2}{*}{256} & 0 & 0,007 & 0,016 & $<0,001$ & 0,007 & 0,016 & $<0,001$ & 0,003 & 0,035 & $<0,001$ \\
& 0,8 & 0,004 & 0,057 & $<0,001$ & 0,002 & 0,071 & $<0,001$ & 0,002 & 0,063 & $<0,001$ \\
& $-0,8$ & 0,003 & 0,008 & $<0,001$ & 0,009 & 0,012 & $<0,001$ & 0,001 & 0,023 & 0,001 \\
\hline
\end{tabular}

Para a função HeaviSine, os resultados podem ser vistos na Figura 6.2 e na Tabela 2. O mesmo padrão geral observado no caso Gaussiano foi observado aqui. É importante notar a maior variabilidade supostamente devida às descontinuidades na função. A superioridade do método iterativo pode ser melhor observada para erros autorregressivos com coeficiente negativo e tamanho de amostra pequeno. Para grandes tamanhos de amostra, ambos os métodos iterativo e Universal têm desempenho similar, enquanto SURE tem maior média de MSE para todos os tamanhos de amostra.

A última função usada nas simulações foi a função Doppler e os resultados são apresentados na Figura 6.3 e na Tabela 3. Ainda o método iterativo proposto possui o mesmo comportamento geral de antes. Para pequenos tamanhos de amostra, os métodos iterativo e Universal apresentam o mesmo desempenho com relação a viés e média de MSE, enquanto que para tamanhos maiores de amostra, SURE tem viés menores mas maior média de MSE. Em geral SURE apresenta maior média de MSE.

Os resultados acima podem ser melhor analisados através das Figuras 6.4 a 6.6. Figura 6.4 mostra resultados de simulação para a função Doppler com erros autorregressivos (coeficiente $\left.\phi_{1}=0,8\right)$. A média do MSE (6.5) para cada um dos três tamanhos de amostra é apresentado juntamente com os os segmentos de linha representando seu desvio padrão amostral. Como esperado, a média do MSE diminui com o aumento do tamanho da amostra, levando a melhores ajustes. Como há autocorrelação 


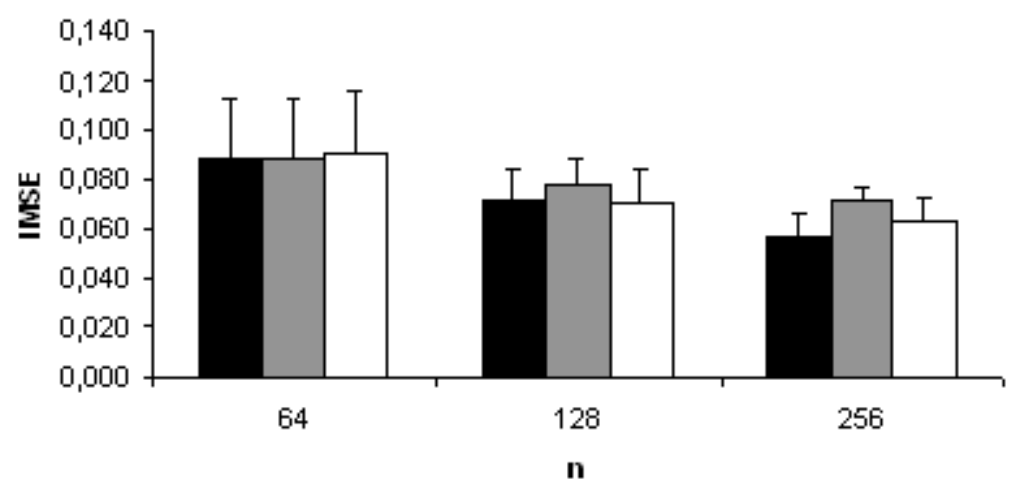

- Iteraivo uUniversal QSure

Figura 6.4: Resultados da simulação para a função Doppler, coeficiente autorregressivo 0,8 , três tamanhos de amostra: média do MSE (6.5), com segmentos de linhas representando seu desvio-padrão amostral.

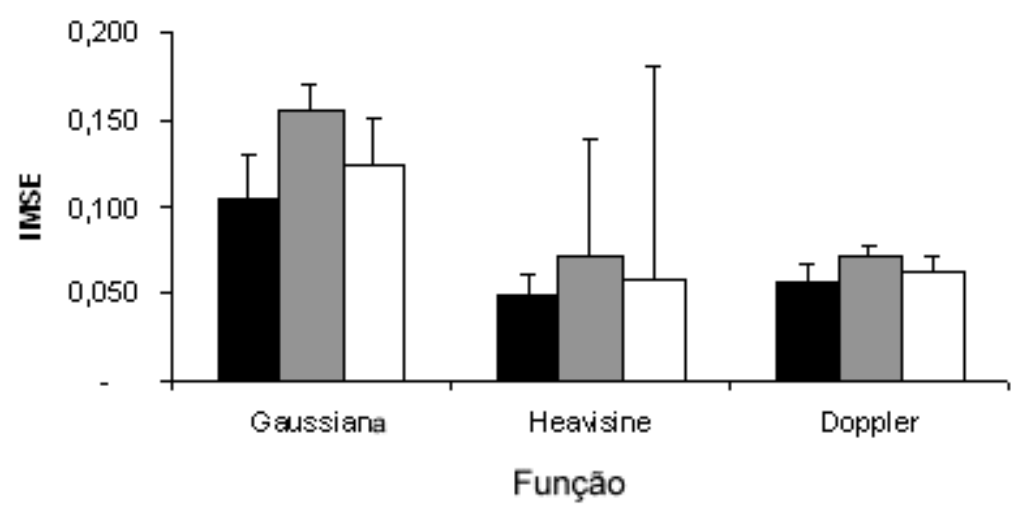

- Iteratiwo uUniversal םSure

Figura 6.5: Resultados da simulação para três funções, coeficiente autorregressivo 0,8 , tamanho de amostra 256: média do MSE (6.5), com segmentos de linhas representando seu desvio-padrão amostral.

positiva, SURE geralmente fornece melhores resultados do que o Universal. Isto também ocorre com outros valores para o coeficiente autorregressivo e com outras funções (veja as tabelas).

Fixando o coeficiente autorregressivo em $\phi_{1}=0,8$ e o tamanho da amostra em $n=256$, então a Figura 6.5 mostra que o método iterativo tem menor média de MSE do que o Universal e do que o SURE, independentemente da homogeneidade da curva. Deve-se notar uma maior variabilidade do MSE para a função HeaviSine, novamente supostamente devida às descontinuidades.

Finalmente, tomando a função Doppler e fixando o tamanho da amostra em 256 observações, Figura 6.6 torna claro um fato superficialmente tocado quando os gráficos das Figuras 6.1, 6.2 e 6.3 foram analisados. Quando os erros são correlacionados, SURE seria esperado fornecer resultados com menor média de MSE do que o Universal, uma vez que a variância dos coeficientes de ondaletas é diferente em cada nível. Entretanto, as simulações mostraram que isso é verdade para coeficientes 


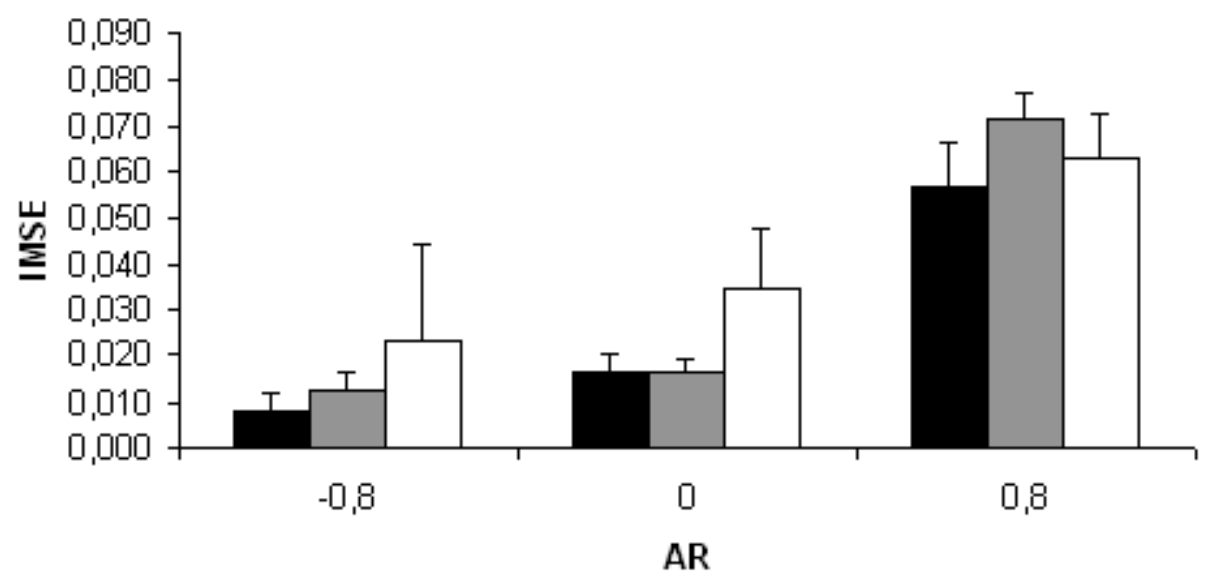

- Iterativo a Universal $\square$ Sure

Figura 6.6: Resultados da simulação para a função Doppler, tamanho amostral 256, três valores do coeficiente autorregressivo: média do MSE (6.5), com segmentos de linhas representando seu desvio-padrão amostral.

autorregressivos maiores que zero; para coeficientes autorregressivos menores que zero, Universal teve um desempenho melhor em termos de média do MSE. Além disso, o método iterativo proposto fornece melhores resultados em qualquer um dos casos. Essa adaptabilidade do método iterativo é apreciada, uma vez que, na prática, pode-se encontrar casos onde não há informação sobre a natureza da autocorrelação.

Na próxima Seção uma breve discussão será feita em torno dos resultados descritos acima.

\subsubsection{Comentários}

$\mathrm{Na}$ última seção foram discutidos alguns resultados das simulações realizadas. Nesta seção serão feitos mais alguns comentários. Eles serão intuitivos e não serão dadas provas formais. Algumas razões para a validade dos resultados principais serão apenas conjecturadas.

Analisando o desempenho dos métodos quanto ao tamanho da amostra, nota-se que o Universal é consistente à medida em que o tamanho da amostra cresce. Ele possui taxas de convergência quase ótimas para uma grande amplitude de classes de funções. O mesmo ocorre com o SURE. Como o método iterativo usa o limiar Universal, é razoável que todos os três métodos sejam equivalentes para grandes amostras. Para amostras pequenas, o método iterativo tem desempenho melhor. Aparentemente isso ocorre porque o método iterativo usa melhor a informação, comparado ao SURE. Talvez um método híbrido (DONOHO; JOHNSTONE, 1995) possa dar resultados ainda melhores.

Analisando o desempenho dos métodos quanto ao sinal da autocorrelação, quando há autocorrelação negativa, a variância dos coeficientes de ondaletas continua sendo diferente para cada um dos níveis. Entretanto, autocorrelações negativas não geram tendências locais, aparentemente fazendo 
com que o SURE trate os erros como se fossem i.i.d. Para os casos com autocorelações negativas, o Universal teve desempenho melhor. Quando há autocorrelação positiva, o SURE apresenta o melhor desempenho, mas o método iterativo tem desempenho próximo ao dele. Quando não há autocorrelação, parece que o Universal e o método iterativo são ligeiramente melhores que o SURE, talvez por causa da esparsidade do sinal em alguns níveis.

No método iterativo proposto, o termo de covariância dos erros é incorporado ao modelo através de um estimador do método dos momentos, independentemente se a autocorrelação é negativa ou positiva. Por sua vez, o sinal é estimado aplicando-se limiares aos coeficientes de ondaletas. No método iterativo, os prós e contras de cada um dos métodos que o compõe parecem ser balanceados, dando origem a um método melhor de modo a alcançar, iterativamente, o mínimo MSE para amostras finitas, independentemente da homogeneidade da função ou do sinal da autocorrelação. Assim, em qualquer caso, o método iterativo proposto se comporta pelo menos tão bem quanto o melhor deles.

Finalmente, embora os resultados apresentados tratem apenas de $S N R=1$, o método iterativo tem um desempenho um pouco melhor do que os apresentados, quando $S N R<1$. Essa vantagem decresce quando $S N R>1$, mas o método iterativo ainda continua apresentando resultados próximos aos do melhor método, independentemente do tamanho da amostra, tipo de função e sinal da autocorrelação.

Uma grande quantidade de métodos de aplicação de limiares tem sido desenvolvida, além do Universal e do SURE. Recentemente, tem sido dada alguma atenção para um método chamado EbayesThresh (JOHNSTONE; SILVERMAN, 2005). Ele usa métodos bayesianos empíricos para tirar vantagem da esparsidade dos coeficientes de ondaletas e para obter limiares melhores. Resultados de simulação com esse método, não apresentados aqui, não alteraram as conclusões gerais acima, uma vez que o EbayesThresh teve desempenho um pouco melhor que o Universal (que é pior do que o SURE em alguns casos) mas ainda pior que o método iterativo proposto.

O método iterativo proposto apresentado aqui usa limiar Universal apenas por simplicidade e popularidade. Qualquer outro método poderia facilmente substituir o Universal. Na realidade, o método proposto é uma alternativa à aplicação de limiares por níveis; não é um método de limiarização novo.

\subsection{Aplicação}

Nesta seção o método iterativo proposto é aplicado a dados financeiros para ilustrar sua utilidade. Uma amostra de tamanho 2048 do índice diário NASDAQ Composite, de 5 de Maio de 1998 a 23 de Junho de 2006 foi usado para motivar a análise. Os respectivos log-retornos têm média zero e uma aparente heteroscedasticidade condicional. A série e os retornos são apresentados nas Figuras 6.7(a) e $6.7(\mathrm{~b})$, respectivamente.

Um modelo autorregressivo de ordem 13 foi ajustado a esses log-retornos. Os resíduos quadráticos deste ajuste são mostrados na Figura 6.7(c). Um modelo $\operatorname{GARCH}(2,1)$, com dois termos ARCH 
(a)

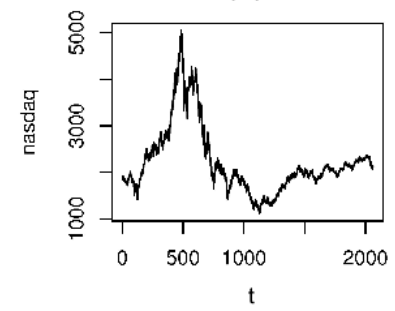

(c)

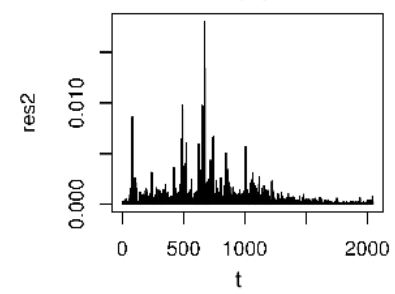

(b)

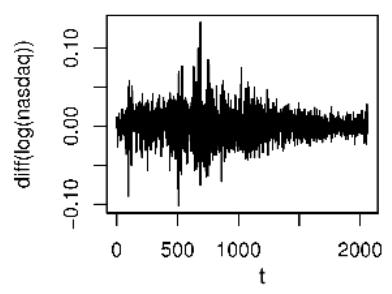

(d)

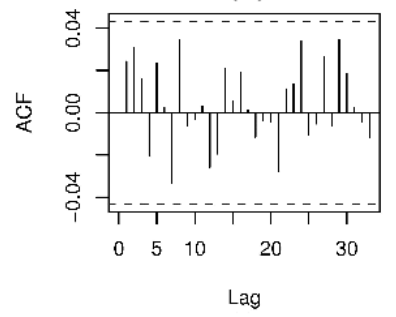

Figura 6.7: (a) Série de 2048 dias do índice da NASDAQ. (b) Log-retornos da série em (a). (c) Resíduos quadráticos de um $\operatorname{AR}(2)$ ajustado à série em (b). (d) Função de autocorrelação amostral dos resíduos de um $\operatorname{GARCH}(2,1)$ ajustado à série em $(\mathrm{c})$.

e 1 termo GARCH, foi ajustado aos resíduos do modelo $\mathrm{AR}(13)$ ajustado, de modo a modelar a volatilidade condicional como a prática comum prescreve. A análise de resíduos desse ajuste GARCH indica que o modelo final AR-GARCH é adequado (Figure 6.7(d)). A equação da volatilidade em um modelo GARCH é determinística mas isso pode não ocorrer na prática. Nestes casos, a volatilidade deveria ser modelada estocasticamente. Tais modelos são frequentemente ajustados na prática por um modelo de volatilidade estocástica, mas para isso seria necessário supor independência dos erros na equação da volatilidade, além de independência dos erros da equação da média. O ajuste GARCH para os dados da Figura 6.7(c) é mostrado na Figure 6.8(a).

Portanto, o método iterativo proposto parece ser apropriado nesta situação. O método iterativo proposto foi aplicado aos resíduos quadráticos do modelo $\mathrm{AR}(13)$ ajustado aos log-retornos da NASDAQ. Neste caso, o modelo (2.10) foi aplicado à volatilidade, representada pelos resíduos quadráticos. A convergência ocorreu em apenas duas iterações. O ajuste GARCH da Figura 6.8(a) é mais suave, mostrando tendências locais e valores menores quando comparado com o ajuste obtido a partir do método iterativo (Figure 6.8(b)).

Heteroscedasticidade condicional ou volatilidade é importante para modelagem macroeconômica (PAGAN; ULLAH, 1999) e financeira (SMITHSON; SMITH JR.; WILFORD, 1995), principalmente nas áreas de precificação de derivativos, gerenciamento de riscos e alocação de ativos (TSAY, 2005). Em Finanças, retornos de ativos são usualmente assumidos como sendo normalmente distribuídos mas várias 

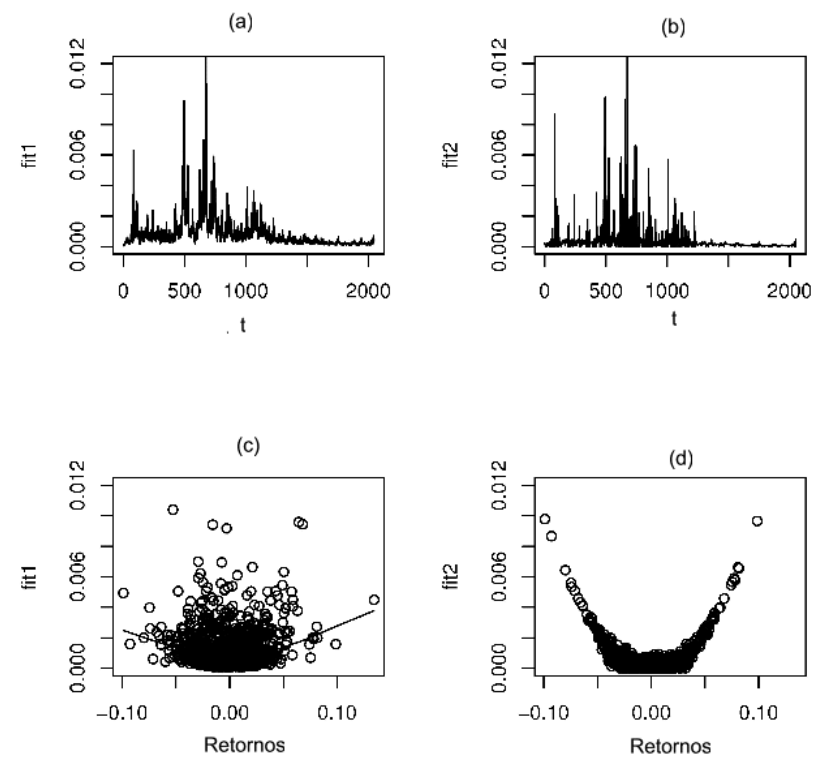

Figura 6.8: (a) Ajuste GARCH para os log-retornos quadráticos da Figura 6.7(c). (b) Ajuste aos dados da Figura 6.7(c) obtido com o método iterativo proposto. (c) Volatilidade condicional do modelo GARCH $(2,1)$ ajustado e curva LOWESS suavizada. (d) Volatilidade condicional do modelo ajustado usando o método iterativo proposto.

pesquisas têm mostrado que eles, na verdade, não são normais. Fatos estilizados sobre distribuições de retornos incluem assimetria à esquerda e excesso de curtose (HARVEY; SIDDIQUE, 1999; PEIRÓ, 1999; PREMARATNE; BERA, 2001). Especificamente, o excesso de curtose (grosseiramente o quarto momento da distribuição) faz com que observações extremas sejam mais prováveis do que no caso normal.

Gráficos da volatilidade estimada contra os retornos comumente mostram alguma curvatura ("sorriso" no jargão de Finanças) (ZIEGELMANN, 2003) indicando excesso de curtose e tornando as assimetrias do mercado mais claras. O efeito de "sorriso torto" da volatilidade é relacionado de perto com a presença de excesso de curtose e assimetria negativa na distribuição dos retornos do ativo subjacente (DAS; SUNDARAM, 1999). Também, assimetria afeta a persistência na variância e tem impactos diferentes na volatilidade porque aumenta a incerteza e o risco.

Análise dessa volatilidade condicional novamente ilumina os menores valores obtidos do modelo GARCH. Na Figura 6.8(c), uma curva suavizada foi obtida pelo método LOWESS. Ele mostra pouco curvatura e nenhuma assimetria para os valores da volatilidade condicional do modelo GARCH. Enquanto isso, o método iterativo proposto mostra que um polinômio de ordem maior poderia ser útil, uma vez que a Figure 6.8(d) mostra muito mais curvatura para a volatilidade condicional obtida a partir do modelo iterativo. Também, os valores do método iterativo são menos variáveis para retornos 
próximos de zero do que seus correspondentes do modelo GARCH. Pouca ou nenhuma assimetria foi observada neste gráfico, indicando que provavelmente modelos assimétricos não são necessários.

Portanto, o método iterativo proposto mostra alguns importantes fatos sobre a volatilidade condicional que estavam escondidos pelo modelo GARCH. Isso provavelmente ocorre por causa da natureza determinística da variância no modelo GARCH (dada toda informação passada), e por causa do tratamento separado dada à estrutura de covariância da volatilidade, no método iterativo. 


\section{Capítulo 7}

\section{Conclusões}

Nesta tese, o interesse foi em obter taxas de convergência a zero, do risco de estimação obtido com os estimadores propostos, quando há erros correlacionados. Quatro métodos de regressão nãoparamétrica via ondaletas, com delineamento desigualmente espaçado foram estudados na presença de erros correlacionados, oriundos de processos estocásticos. Foram apresentadas condições sobre os erros e adaptações aos procedimentos necessárias à obtenção de taxas de convergência quase minimax, para os estimadores. Sempre que possível foram obtidas taxas de convergência para os estimadores no domínio da função, sob condições bastante gerais a respeito da função a ser estimada, do delineamento e da correlação dos erros. Todos os métodos estudados apresentaram vantagens e desvantagens em relação a cada um desses tópicos. Mediante estudos de simulação, foram avaliados os comportamentos de alguns métodos propostos quando aplicados a amostras finitas. Em geral sugere-se usar um dos procedimentos estudados mas aplicando limiares aos coeficientes de detalhes por níveis. Como a estimação da variância desses coeficientes pode ser problemática em alguns casos, também se propôs um procedimento iterativo semi-paramétrico geral para métodos que utilizam ondaletas, na presença de erros em séries temporais.

Quando o delineamento é fixo, para uma classe bastante geral de funções e erros gaussianos com covariância limitada superiormente pela variância, a aplicação do método de Cai e Brown (1998) com limiares por níveis, apresenta taxa de convergência quase minimax em relação ao caso i.i.d., utilizandose ondaletas de suporte compacto. Destaca-se também a descoberta que isso ocorre mesmo quando os erros possuem memória longa.

No caso de delineamento aleatório jittered ou seguindo uma distribuição Uniforme [0,1], para a mesma classe de funções do caso de delineamento fixo, mas com erros gaussianos com autocovariâncias absolutamente somáveis, mostrou-se que a aplicação dos métodos usuais de ondaletas com limiares por níveis, apresenta taxa de convergência quase minimax, utilizando-se ondaletas de suporte compacto. Destacam-se também os teoremas que ilustram como tais erros podem ser gerados a partir de um processo $\operatorname{AR}(1)$ em tempo contínuo.

Para delineamentos aleatórios gerais com densidade de suporte compacto, para uma ampla classe de funções e sob razoáveis condições em relação ao processo estocástico gerador dos erros, tanto 
o método que usa ondaletas deformadas quanto o que usa ondaletas adaptativas, suaves ou não, apresentam boas taxas de convergência. No caso de ondaletas deformadas, exige-se normalidade dos erros mas a classe de funções é bastante geral. Com ondaletas adaptativas, pode-se abrir mão da normalidade, mas os resultados restringem-se ao domínio das ondaletas.

Todos os procedimentos desenvolvidos para delineamentos com espaçamento desigual podem ainda servir de base para o procedimento semi-paramétrico proposto. Dessa forma, evita-se a estimação da variância dos coeficientes de detalhes dos níveis mais grossos, ao preço de um maior tempo computacional.

Há diversas possibilidades para pesquisas futuras como comparações entre os métodos, estudo do comportamento sob outras funções de limiar, outros métodos de estimação dos coeficientes de ondaletas, etc. Porém, a extensão desta tese para modelos de regressão com mais de uma variável explicativa é interessante, dentre outros motivos, por causa da chamada "maldição da dimensionalidade". Também interessante e em desenvolvimento é o estudo teórico das propriedades do procedimento semi-paramétrico onde uma das dificuldades origina-se de sua natureza algorítmica. 


\section{Referências Bibliográficas}

ALTMAN, N. S. Kernel smoothing of data with correlated errors. Journal of the American Statistical Association, v. 85, p. 749-759, 1990.

BENTKUS, R. Rate of uniform convergence of statistical estimators of spectral density in spaces of differentiable functions. Lithuanian Math. Journal, v. 26, p. 209-219, 1985.

BIERENS, H. Uniform consistency of kernel estimators of a regression function under generalized conditions. Journal of the American Statistical Association, v. 78, p. 699-707, 1983.

CAI, T. T. Nonparametric function estimation via wavelets. Tese (Doutorado) - Cornell University, 1996.

CAI, T. T.; BROWN, L. Wavelet shrinkage for nonequispaced samples. The Annals of Statistics, v. 26, n. 5 , p. $1783-1799,1998$.

CAI, T. T.; BROWN, L. Wavelet estimation for samples with random uniform design. Statistics and Probability Letters, v. 42, p. 313-321, 1999.

CHESNEAU, C.; WILLER, T. Numerical performances of a warped wavelet estimation procedure for regression in random design. 2007. Http://www.math.jussieu.fr/ willer/files/regression.pdf e http://hal.archives-ouvertes.fr/docs/00/13/38/31/PDF/regression.pdf.

CLERC, M.; MALLAT, S. Estimating deformations of stationary processes. Annals of Statistics, v. 31, p. $1772-1821,2003$.

COCHRANE, D.; ORCUTT, G. H. Applications of least squares regression to relationships containing autocorrelated errors. Journal of the American Statistical Association, v. 44, n. 245, p. $32-61,1949$.

DAS, S.; SUNDARAM, R. Of smiles and smirks: a term structure perspective. Journal of Financial and Quantitative Analysis, v. 34, p. 211-239, 1999.

DAUBECHIES, I. Ten Lectures on Wavelets. Philadelphia, Pennsylvania: SIAM, 1992. (CBMS-NSF Regional Conference Series in Applied Mathematics).

DAVIDSON, J. Stochastic Limit Theory. New York: Oxford University Press, 1994.

DELOUILlE, V. Nonparametric Stochastic Regression Using Design-adapted Wavelets. Tese (Doutorado) - Universite Catholique de Louvain, 2002. Institut de Statistique.

DIPPÉ, M. A. Z.; WOLD, E. H. Antialiasing through stochastic sampling. ACM SIGGRAPH Computer Graphics, v. 19, n. 3, p. 69-78, 1985. 
DONOHO, D. L.; JOHNSTONE, I. M. Ideal spatial adaptation via wavelet shrinkage. Biometrika, v. 81, p. $425-455,1994$.

DONOHO, D. L.; JOHNSTONE, I. M. Adapting to unknown smoothness via wavelet shrinkage. Journal of the American Statistical Association, v. 90, p. 1200-1224, 1995.

DOUKHAN, P. Mixing: Properties and Examples. New York: Springer-Verlag, 1994. (Lecture Notes in Statistics, v. 85).

FLANDRIN, P. Time-scale analyses and self-similar stochastic processes. In: BYRNES, J. S. (Ed.). Wavelets and Their Applications. Dordrecht: Kluwer Academic, 1994. p. 121-142.

FULLER, W. A. Introduction to Statistical Time Series. Second. New York: Wiley, 1996.

GIRARDI, M.; SWELDENS, W. A new class of unbalanced haar wavelets that form an unconditional basis for $l_{p}$ on general measure spaces. J. Fourier Anal. Appl., v. 3, n. 4, p. 457-474, 1997.

GRADSHTEǏN, I. S. et al. Table of Integrals, Series, and Products. Seventh. Amsterdan: Academic Press, 2007.

HÄRDLE, W. et al. Wavelets, Approximation, and Statistical Applications. New York: Springer, 1998. (Lecture Notes in Statistics, v. 129).

HARVEY, C. R.; SIDDIQUE, A. Autoregresive conditional skewness. Journal of Financial and Quantitative Analysis, v. 34, p. 465-487, 1999.

HECKMAN, J.; POLACHECK, S. Empirical evidence of functional form of the earnings-schooling relationship. Journal of the American Statistical Association, v. 69, p. 350-354, 1974.

HEINONEN, J. Lectures on lipschitz analysis. Lectures at the 14th Jyväskylä Summer School, http://www.math.jyu.fi/research/reports/rep100.pdf (Mar 2007). August 2004.

JOHNSON, N. L.; KOTZ, S.; BALAKRISHNAN, N. Continuous Univariate Distributions. 2. ed. New York: Wiley, 1995.

JOHNSTONE, I. M.; SILVERMAN, B. W. Wavelet threshold estimators for data with correlated noise. Journal of the Royal Statistical Society Series B Statistical Methodology, v. 59, n. 2, p. 319-351, 1997.

JOHNSTONE, I. M.; SILVERMAN, B. W. Empirical bayes selection of wavelet thresholds. The Annals of Statistics, v. 33, n. 4, p. 1700-1752, 2005.

KERKYACHARIAN, G.; PICARD, D. Thresholding algorithms, maxisets and well-concentrated bases. Test, v. 9, n. 2, p. 283-345, 2000.

KERKYACHARIAN, G.; PICARD, D. Non-linear approximation and Muckenhoupt weights. [S.1.], 2002.

KERKYACHARIAN, G.; PICARD, D. Regression in random design and warped wavelets. Bernoulli, v. 10, n. 6 , p. $1053-1105,2004$.

KERKYACHARIAN, G.; PICARD, D. Non-linear approximation and muckenhoupt weights. Constructive Approximation, v. 24, n. 2, p. 123-156, 2006. 
KUTNER, M. H. et al. Applied Linear Statistical Models. 5. ed. New York: McGraw-Hill/Irwin, 2004.

Le Pennec, E.; MALLAT, S. Sparse geometric image representation with bandelets. 2003.

Http://www.cmap.polytechnique.fr/ mallat/biblio.html.

LEADBETTER, M. R.; LINDGREN, G.; ROOTZÉN, H. Extremes and Related Properties of Random Sequences and Processes. New York: Springer, 1986.

MORETtin, P. A. Ondas e Ondaletas. São Paulo: Editora da Universidade de São Paulo, 1999.

NASON, G.; KOVAC, A.; MAECHLER, M. Wavethresh - Software to perform wavelet statistics and transforms. $R$ package version 2.2-9. [S.1.], 2006. Http://www.R-project.org.

NEUMANN, M. H.; SACHS, R. von. Wavelet thresholding: Beyond the gaussian i.i.d. situation. In: ANTONIADIS, A.; OPPENHEIM, G. (Ed.). Wavelets and Statistics. New York: Springer-Verlag, 1995, (Lecture Notes in Statistics, v. 103). p. 301-329.

OPSOMER, J.; WANG, Y.; YANG, Y. Nonparametric regression with correlated errors. Statistical Science, v. 16, n. 2, p. 134-153, 2001.

PAGAN, A.; ULlAH, A. Nonparametric Econometrics. Cambridge: Cambridge University Press, 1999.

PEIRÓ, A. Skewness in financial returns. Journal of Banking and Finance, v. 23, p. 847-862, 1999.

PREMARATNE, G.; BERA, A. K. Modeling Asymmetry and Excess Kurtosis in Stock Return Data. [S.1.], 2001. v. 23.

QIN, D.; GILBERT, C. L. The error term in the history of time series econometrics. Econometric Theory, v. 17, n. 2, p. 424-450, 2001.

R Development Core Team. R: A language and environment for statistical computing. Vienna, Austria, 2005. ISBN 3-900051-07-0, http://www.R-project.org (3 Jul 2006).

SARDY, S. et al. Wavelet shrinkage for unequally spaced data. Statistics and Computing, v. 9, p. 65-75, 1999.

SIMOENS, J.; VANDEWALLE, S. A stabilized lifting construction of wavelets on irregular meshes on the interval. SIAM Journal on Scientific Computing, v. 24, n. 4.

SMITHSON, C. W.; SMITH JR., C. W.; WILFORD, D. S. Managing Financial Risk. New York: Irwin, 1995.

SWELDENS, W. The lifting scheme: A construction of second generation wavelets. SIAM Journal of Mathematical Analysis, v. 29, n. 2, p. 511-546, 1997.

TSAY, R. S. Analysis Of Financial Time Series. New York: Wiley, 2005.

WHITCHER, B. Waveslim: basic wavelet routines for one-, two- and three-dimensional signal processing. $R$ package version 1.5. [S.1.], 2005. Http://www.image.ucar.edu/staff/whitcher/ and http://www.image.ucar.edu/staff/whitcher/book/ (Jul 2006). 
WUERTZ, D. Rmetrics - The Dynamical Process Behind Markets. [S.1.], 2006. R package, version 240.10068, collection LongRangeDependence, http://www.R-project.org.

ZIEGELMANN, F. A. Nonparametric and semi-parametric methods in time series: the kernel smoothing approach. August 2003. Mini-course presented at the 10th ESTE - Escola de Séries Temporais e Econometria, São Pedro, SP, Brazil. P. 11-13. 